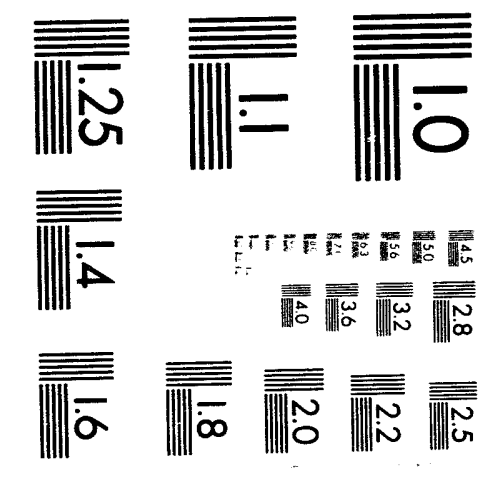



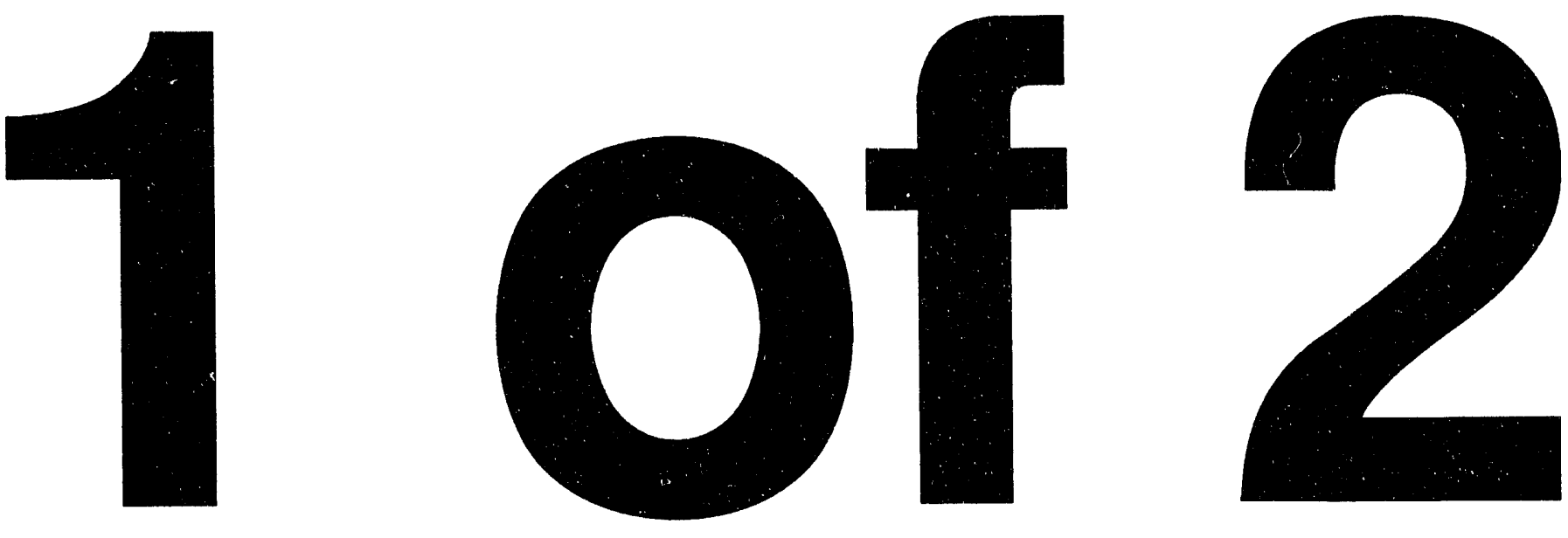


\section{ANL-EP-TM-93/1}

Argonne National Laboratory

9700 South Cass Avenue

Argonne, Illinois 60439

\section{SOLIDS FLOW RATE MEASUREMENT IN DENSE SLURRIES}

Karl G. Porges and Ezzat D. Doss

Engineering Physics Division

September 1993

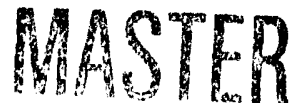




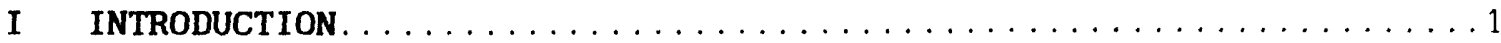

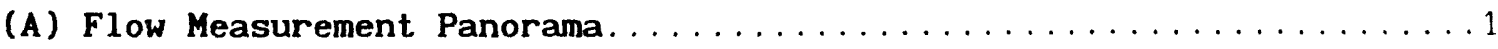

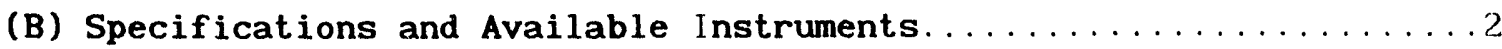

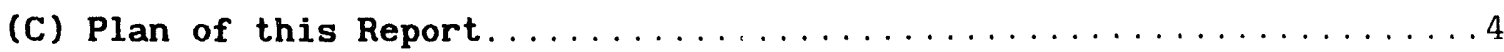

II ABSOLUTE READOUT VS CALIBRATION IN DENSE-SLURRY FLOW MEASUREMENT $\ldots 6$

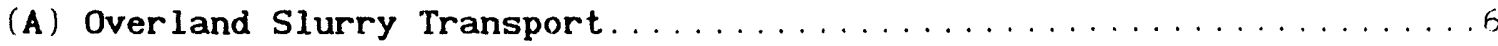

(B) In-Plant Solids Conveyance $\ldots \ldots \ldots \ldots \ldots \ldots \ldots \ldots \ldots \ldots \ldots$

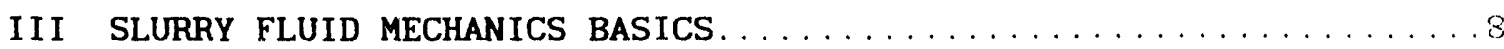

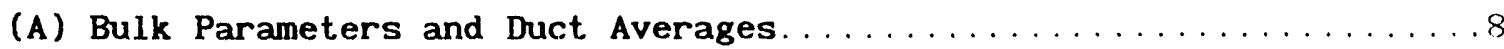

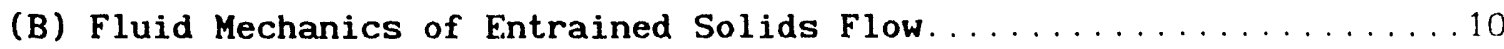

(C) Velocity Map for laminar non-Newtonian Flow in a Square Duct....12

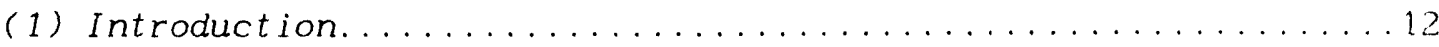

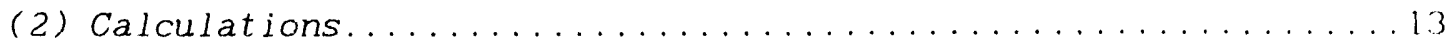

(a) Velocity Map for Newtonian Flow....................14

(b) Velocity Map for Power-law (Pseudoplastic) Flow...........15

(c) Additional Rows \& Columns needed for I teration............17

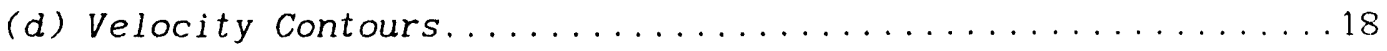

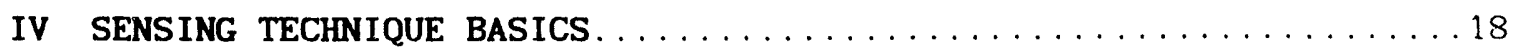

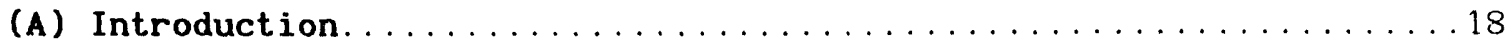

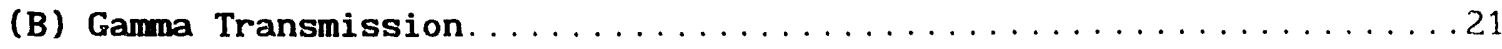

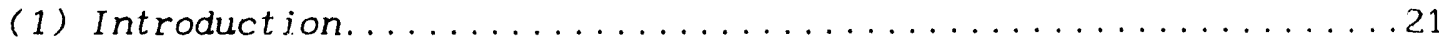

(2) Gamma Transmission: Homogeneous, Uniform Medium...........26

(3) Transmission through Quasi-homogeneous Binary Mixtures........26

(4) Extension to Three-phase Quasi-homogeneous Media............28

(5) Data Processing Options............................ 29

(6) Effect of Non-Uniform Cross-Beam Phase distributions.......... 30

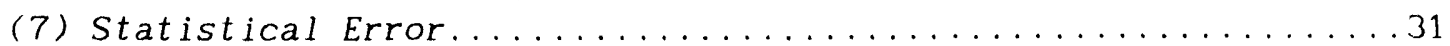

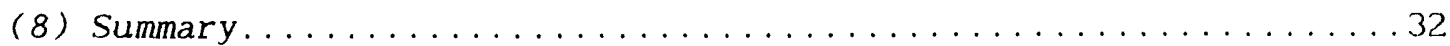




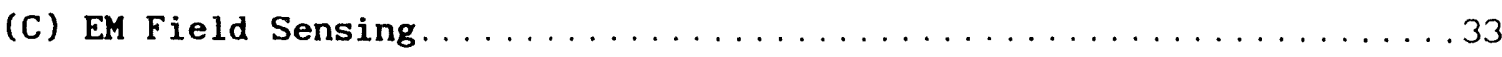

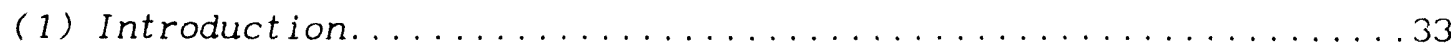

(2) Input Circuit: Dielectric Constant and Readout Signal........34

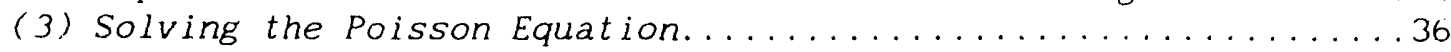

(4) Equipotentials for Representative Electrode Geometries.........38

(a) ANL Spoolpiece \# 1 (External Parallel-Plate E Shims)........38

(b) Wrapped-Plate Electrodes.......................43

(c) "Patch" Signal Electrode.......................44

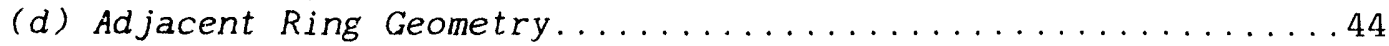

(e) Helical Strip Electrodes.........................45

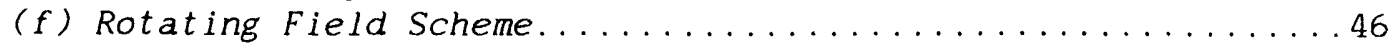

(g) Mixed-Geometry Scheme.........................46

(h) ANL Spoolpiece \#2 (Wrapped-Plate with Guard Strips).......48

(5) Local Field Strength and Sensing Efficiency................49

(6) The Bulk Dielectric Constant of Binary Mixtures.............51

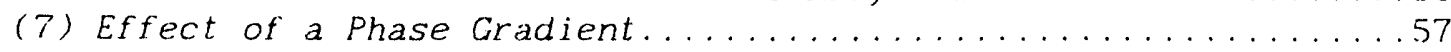

(D) Statistics associated with Solid Fraction Fluctuations......... 1

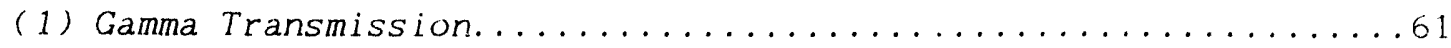

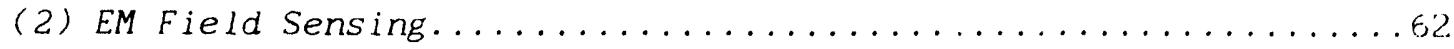

(3) Error Removal through Short Sampling.................62

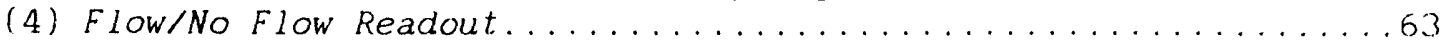

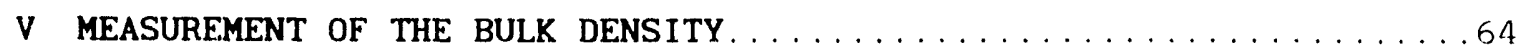

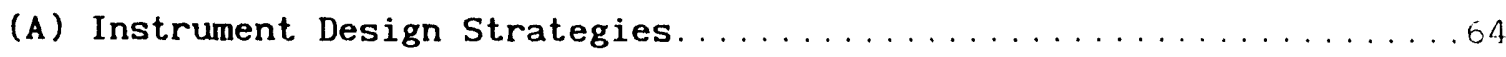

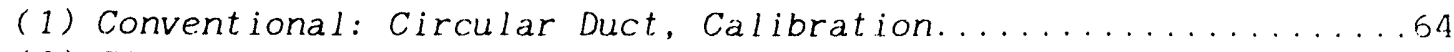

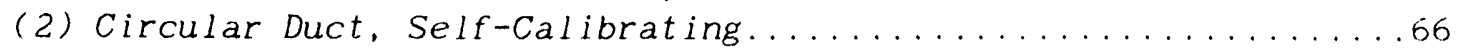

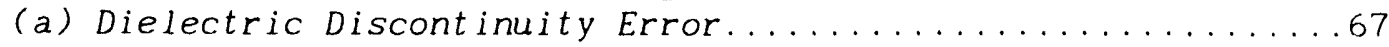

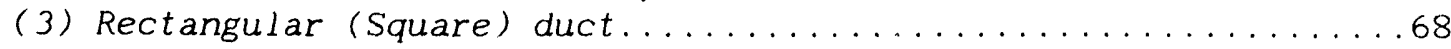

(a) Transition Piece Design..........................68

(b) Dummy Duct and Pressure Envelope....................69

(4) Pipeline On-Line Densitometry Design Strategies............69

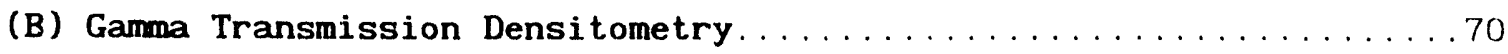

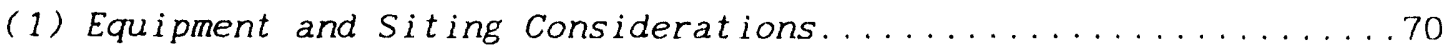

(2) Electronics: Light Pulser Stabilization.................71

(3) Calculations and Measurement of Solids Intrinsic Density.....72

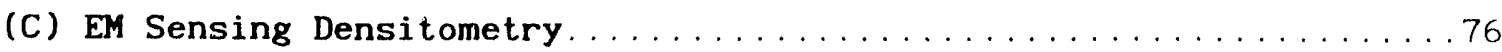

(1) Hardware Design Notes......................... 76

(2) Electronics: Channel Switching Stabilization.............. 


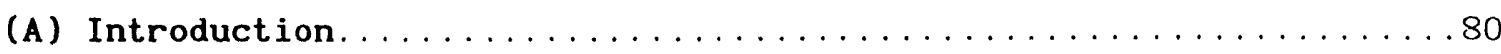

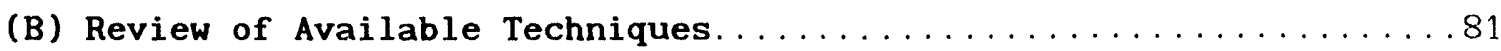

(1) Acoust ic Diagonal-Path and Acoustic-Doppler.............81

(2) Magnetic FM and Coriolis' Force FM...................85

(3) Radioactive Particle Injection and Pulsed Neutron Activation. . 86

(4) Cross-Correlation Flow Measurement....................88

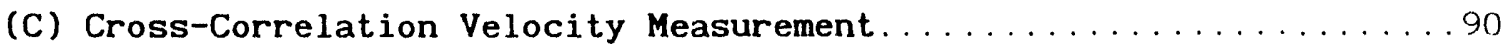

(1) Historical Review and present Status..................90

(2) Underlying Processes and Data Processing in CCF and PNA......91

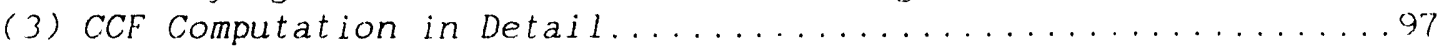

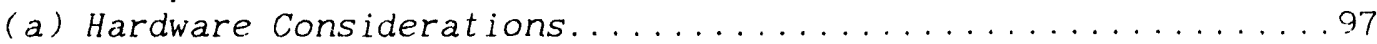

(b) Numerical Examples; comparison to FFT.................98

(c) Calculation of the Average Velocity. Turbulent Flow.......100

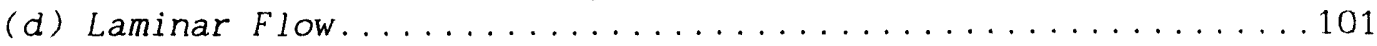

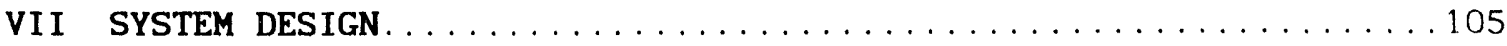

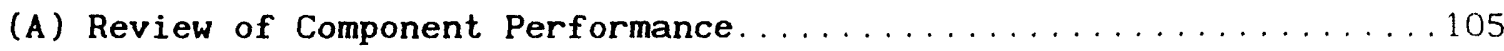

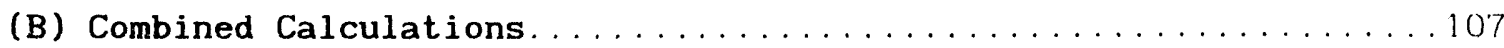

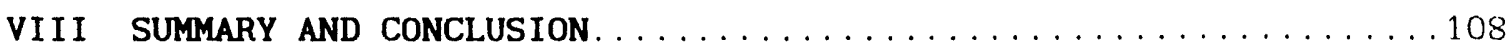

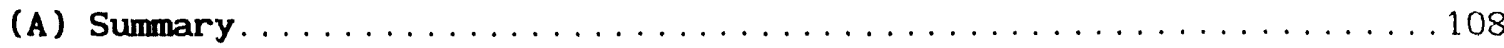

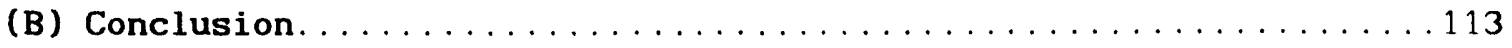

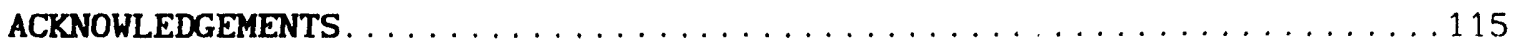

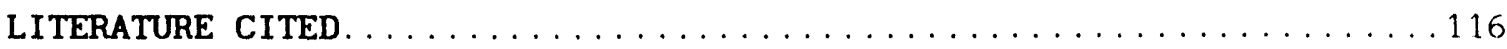

APPENDIX A:

INTRINSIC STATISTICAL ERROR IN CROSS-CORRELATION-BASED MEASUREMENT . . 127

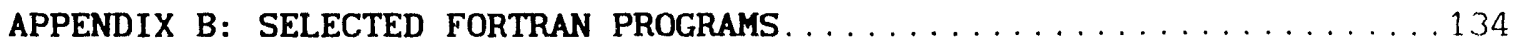

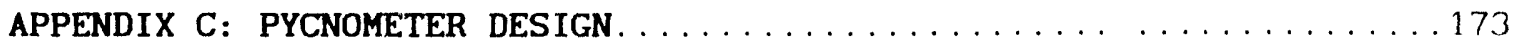




\section{I S T O F F I G U R E S}

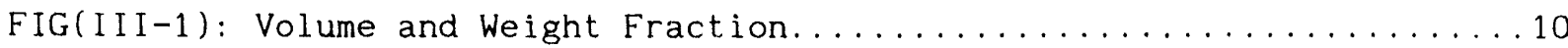

FIG(III-2a): Velocity Contours, Square Duct, Newtonian-Laminar Flow $(n=1.0) 19$

FIG(III-2b): Velocity Contours, Square Duct, Pseudoplastic Flow $(n=.8) \ldots \ldots 19$

FIG(III-2c): Velocity Contours, Square Duct, Pseudoplastic Flow $(n=.6) \ldots \ldots 19$

FIG(III-2d): Velocity Contours, Square Duct, Pseudoplastic Flow $(n=4) \ldots 19$

FIG(III-3): "Specific" Velocity Contours $(n=.4$ to 1.0$) \ldots \ldots \ldots \ldots$

FIG(IV-1): Correction Parameters $\alpha$ and $\beta$ vs Atomic Number $z(661 \mathrm{keV}) \ldots 26$

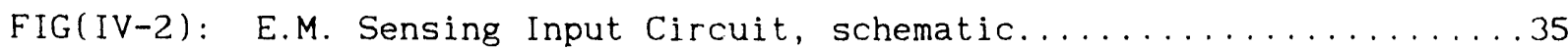

FIG(IV-3): Equipotentials and Field Lines near the edges of a Paralle1-

Plate Capacitor, via Schwartz Transformation.............. 37

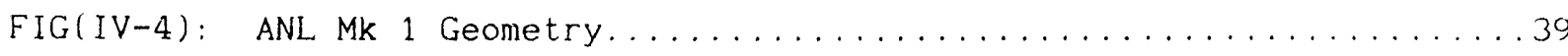

FIG(IV-5): Series Capacity associated with ANL Mk 1, schematic......41

FIG $(I V-6):$ Equipotentials, ANL Mk 1 , for $x_{w}=4.00 \ldots \ldots \ldots \ldots \ldots \ldots \ldots$

FIG(IV-7): ANL Mk 1 Equipoientials calculated for $\kappa_{\mathrm{m}}=60.00 \ldots \ldots 42$

FIG(IV-8): Wrapped-Plate Geometry Equipotentials..............43

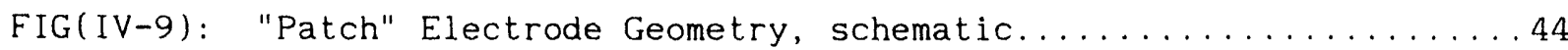

FIG(IV-10): "Adjacent-Ring" Electrode Geometry, schematic..........45

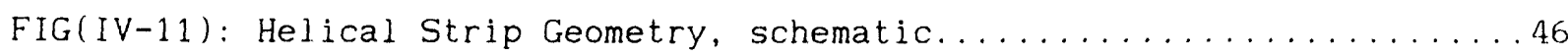

FIG(IV-12): Equipotentials for Helical Strip Electrode Geometry.......47

FIG(IV-13): Pseudo-Equipotentials for "Mixed" Geometry.............48

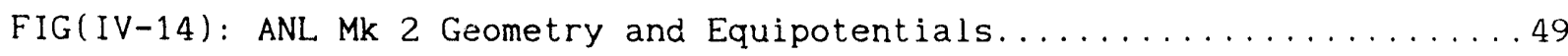

FIG(IV-15): "Cell" Contribution to the Signal, schematic...........50

FIG(IV-16): Binary Mixture Diel. Constant Model (Maxwell), schematic.....53

FIG(IV-17): $\kappa_{\mathbf{m}}$ Non-Linearity vs. $\phi_{\mathbf{s}}$ for different Models:

$\kappa_{\mathbf{s}}=6.0, \boldsymbol{\kappa}_{\mathrm{f}}=60 \ldots \ldots \ldots \ldots \ldots \ldots \ldots \ldots \ldots \ldots \ldots \ldots \ldots \ldots \ldots \ldots$

FIG(IV-18): $\kappa_{\mathbf{m}}$ Non-Linearity vs. $\phi_{\mathbf{s}}$ for different Models:

$$
\kappa_{\mathbf{s}}=6.0, \boldsymbol{\kappa}_{\mathrm{f}}=2.5 \ldots \ldots \ldots \ldots \ldots \ldots \ldots \ldots \ldots \ldots \ldots \ldots \ldots \ldots \ldots \ldots
$$

FIG(IV-19): Layered Phase Separation Model, schematic............. 58

FIG(IV-20): Bulk Density Readout Error for coal/oil slurry, weighted and unweighted average of Horizontal and Vertical Plate sets... 60

FIG(IV-21): Bulk Density Readout Error for coal/water slurry, weighted and unweighted average of Horizontal and Vertical Plate sets 60 
FIG(V-1): Bench-Top Test Loop for EM Densitometer Calibration........ 66

FIG(V-2): Effect of Dielectric Discontinuity, Schematic...........67

FIG $(V-3)$ : Isometric View, Transition Piece, Circular to Square.......70

FIG(V-4): Design Details, ANL 1.1k 2 EM Sensing Spoolpiece, Circ. Duct....76

FIG(V-5): Design Details, EM Sensing Spoolpiece for Square Duct........77

FIG(VI-1): Acoustic Diagonal Path Scheme, schematic.............. 82

FIG(VI-2): CCF Computation Hardware, Schematic................. 99

FIG(VI-3): Field-Adjustment Scheme Equipotentials and Field Lines...... 102

FIG(VI-4): Overlap of Field-Line-Selected Regions and Velocity Contours 103

FIG(VI-5): Driving Circuit and Input Amplifiers, Contour-Select Scheme 104

\section{T A B L E S}

TABLE $(I-1):$ REQUIREMENTS, DENSE-SLURRY FLOW MEASUREMENT $\ldots \ldots \ldots \ldots \ldots \ldots 2$

TABLE $(I-2): \quad$ INGREDIENTS OF ABSOLUTE READING $\ldots \ldots \ldots \ldots \ldots \ldots \ldots \ldots \ldots$

TABLE $(I-3): \quad$ FM ELIMINATED BY SPEC $2 \ldots \ldots \ldots \ldots \ldots \ldots \ldots \ldots \ldots \ldots \ldots \ldots \ldots \ldots \ldots$

$\operatorname{TABLE}(I-4): \quad$ FM QUALIFYING UNDER SPEC $2 \ldots \ldots \ldots \ldots \ldots \ldots \ldots \ldots \ldots \ldots$

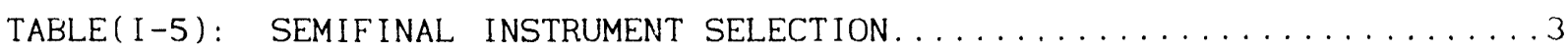

TABLE (I I-1): FLOW MEASUREMENT SPECIFICATIONS FOR SLURRY LINES $\ldots \ldots \ldots \ldots$

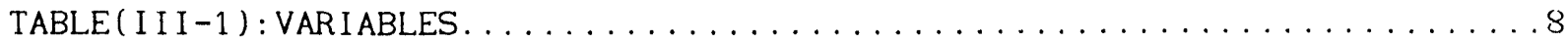

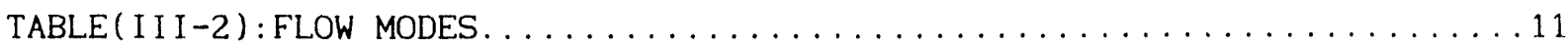

TABLE (IV-1): $661 \mathrm{keV}$ PHOTON REMOVAL CROSS SECTIONS AND DERIVED

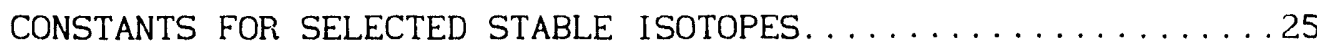

TABLE $(V-1): \quad$ COMPUTATION FACTORS FOR MAJOR CONSTITUENTS OF OHIO \# 9 COAL AND TWO FLUIDS............................ 74

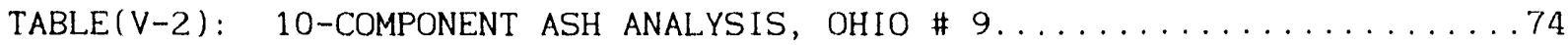

TABLE $(V-3):$ TERMS OF EQ. (IV-10) FOR OHIO \# 9 COAL $\ldots \ldots \ldots \ldots \ldots \ldots \ldots 74$

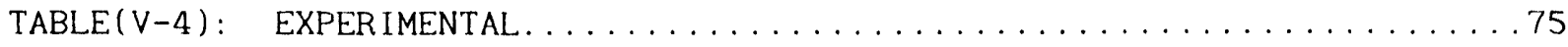

TABLE $(V-5)$ : COMPUTATION FACTORS FOR MAJOR CONST ITUENTS OF C-15868 (INLAND)

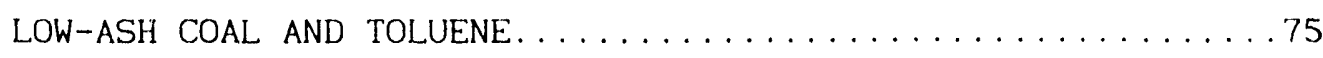


TABLE(VI-1): COMMERCIALLY AVAILABLE, NON-INTRUSIVE FLOWMETERS . . . . . . . 81

TABLE(VI-2): SPECIAL FLOW MEASUREMENT TECHIQUES $\ldots \ldots \ldots \ldots \ldots \ldots \ldots \ldots$

TABLE(VII-1): PERFORMANCE CHARACTERISTICS, DENSITOMETRY $\ldots \ldots \ldots \ldots$

TABLE(VII-2) : PERFORMANCE CHARACTERISTICS, VELOCITY MEASUREMENT $\ldots \ldots 106$

TABLE(VIII-1): CONDITIONAL SPECIFICATIONS FOR FLOW MEASUREMENT IN DENSE,

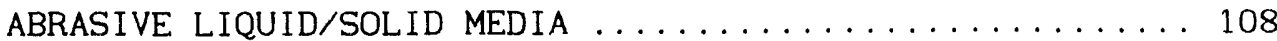

TABLE(VIII-2): INSTRUMENT SPECIFICATIONS $\ldots \ldots \ldots \ldots \ldots \ldots \ldots \ldots \ldots$

TABLE(VIII-3): TASKS (A) AND (B): ACCEPTABLE DEVICES $\ldots \ldots \ldots \ldots \ldots \ldots 110$

TABLE(VIII-4): GAMMA TRANSMISSION DENSITOMETRY $\ldots \ldots \ldots \ldots \ldots \ldots \ldots \ldots$

TABLE(VIII-5): E.M. FIELD SENSING DEVICES $\ldots \ldots \ldots \ldots \ldots \ldots \ldots \ldots \ldots$

TABLE(VIII-6) : E.M.FIELD SENSING CHARACTERISTICS,

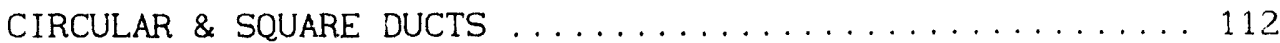




\title{
SOLIDS FLOW RATE MEASUREMENT IN DENSE SLURRIES
}

\author{
Karl G. Porges and Ezzat D. Doss
}

\begin{abstract}
Accurate and rapid flow rate measurement of solids in dense slurries remains an unsolved technical problem, with important industrial applications in chemical processing plants and long-distance solids conveyance. In a hostile two-phase medium, such a measurement calls for two independent parameter determinations, both by non-intrusive means. Typically, dense slurries tend to flow in laminar, nonNewtonian mode, eliminating most conventional means that usually rely on calibration (which becomes more difficult and costly for high pressure and temperature media). These issues are reviewed, and specific solutions are recommended in this report. Detailed calculations that lead to improved measuring device designs are presented for both bulk density and average velocity measurements. Cross-correlation, chosen here for the latter task, has long been too inaccurate for practical applications. The cause and the cure of this deficiency are discussed using theory-supported modeling.

Fluid Mechanics are used to develop the velocity profiles of laminar non-Newtonian flow in a rectangular duct. This geometry uniquely allows the design of highly accurate 'capacitive' devices and also lends itself to gamma transmission densitonietry on an absolute basis. An absolute readout, though of less accuracy, is also available from a capacitive densitometer and a pair of capacitive sensors yields signals suitable for cross-correlation velocity measurement.
\end{abstract}




\section{INTRODUCTION}

\section{(A) Flow Measurement Panorama}

The Rate of Flow is one of the most widely measured variables - $\pi \alpha \nu \tau \alpha \rho \varepsilon \iota$ $\kappa \alpha \iota \mu \varepsilon \tau \rho \iota \kappa \eta \nu \varepsilon \chi \varepsilon \iota(*)$ as Heraclitus might well have said - in Commerce and Industry, in the US and Worldwide: from gas and water meters in urban dwellings to gas and water mains, sewage pipes, irrigation water flow in open channels and finally, to complex piping systems in materials fabrication plants, power plants and petroleum refineries, instruments of great variety are used to measure and record flow. Responding to demand, the Instrumentation industry currently offers flow meters designed for installation in channels and ducts of almost arbitrary size, flow meters based on 200 year-old design and others based on recent patents, flow meters equipped with dial and needle indication and flow meters with digital-remote readout, directly linked to computers. With regard to accuracy, absolute-reading instruments are available for clean fluid service. Other types of flow meter require calibration, in which the instrument and a secondary Standard are mounted on a Test loop and compared (the Secondary Standard, in turn, being certified by comparison to a NIS Standard).

Against this background of well-established, smoothly functioning technology, there remain some scenarios where a serious need for accurate Flow measurement exists, yet cannot be met by available equipment. These situations occur chiefly in the small but economically important segment of the Chemical Industry that processes solid raw materials or intermediary products. Conveyor belts, screws and other mechanical means, long used to move granular solids within plants, are prone to failure as well as objectionable for environmental reasons; solids entrainment by a gas or liquid seems a better choice. To introduce solid reagents into a Chemical Reactor, typically at high temperature and pressure, passing a pre-heated slurry through a compressor-pump is especially preferable to other means. For long-distance solids movement, vehicular transport by road or rail has been the standard technique. The alternative use of pipelines has been much studied and occasionally implemented(I-1). Useful though slurry conveyance appears to be in the cited applications, it has its own problems: at the large solids concentration mandated by economic considerations, media are highly erosive and abrasive. Among other consequences, this has thus far defeated efforts to provide Instrumentation that can support efficient management of slurry conveyors and, in particular, is indispensable for chemical reagent balance in a reactor solids feedline.

Specifications that measurement devices and systems must meet vary considerably with the application. For example: the solid fraction varies much less in pipeline than in in-plant conveyance; the entraining fluid is inevitably water for the former but tends to vary for the latter; a measurement of the overall Flow rate suffices for pipeline operation but is needed at many points along the line, while the object of measurement for in-plant conveyance is the solids feed rate, requiring two independent instruments. Duct diameters of $0.5-1.5 \mathrm{~m}$ are a major problem for pipeline instrumentation; in-plant conveyors are of more manageable size. As has been widely recognized (and emphasized in reports on slurry conveyor instrumentation), all dense-slurry conveyor Flow meters must be non-intrusive. This eliminates many existing measure-

(*) Everything Flows and Flow wants to be Measured. 
ment devices and schemes that have been successfully employed under more benign circumstances. Considerations of instrumentation operation and management suggest yet another difficult specification: an absolute readout capability, important in proportion to the difficulty of providing calibration when the medium at the measurement site is not only abrasive, but at high temperature and pressure, and/or when the conveyed solid tends to vary. Both conditions are commonly encountered in coal conversion and combustion plants.

The practical origin of these problems, which seem to us a compelling reason for specifying an absolute readout capability, will be more thoroughly discussed in the following. An absolute readout capability implies a sufficiently detailed understanding of flow-related and instrument-related processes, available for some instruments but not for others, and thus further shortens the list of candidate Flow measurement schemes. The next section summarizes the selection process, beginning with a list of Specifications and instruments but stopping short of absolute readout, whose evaluation is the main subject of the remainder of this report. There is no intention to disparage calibration when it is convenient and practical; neither is the case in slurry feed lines.

\section{(B) Specifications and Available Instruments}

The Specifications introduced in the foregoing are summarized in TABLE (I-1), below. Absolute Reading ingredients are listed in TABLE $(I-2)$.

TABLE $(I-1)$ : REQUIREMENTS, DENSE-SLURRY FLOW MEASUREMENT

\begin{tabular}{|l|l|}
\hline 1 & Two independent variables must be measured \& combined by on-line computer \\
\hline 2 & Both measurements must be made by non-intrusive means \\
\hline 3 & Instrument readouts must allow inference of result without calibration \\
\hline
\end{tabular}

TABLE (I-2): INGREDIENTS OF ABSOLUTE READING

\begin{tabular}{|r|l|}
\hline (a) & The measurement itself, on-line, sensing the 2-phase medium \\
(b) & A fluid-phase reference measurement with identical sensor, geometry etc. \\
(c) & Other information, such as: \\
\hline i & Off-line (laboratory) analysis of samples of both phases \\
\hline ii & Tables of physical constants \\
\hline iii & A set of valid equations connecting sensed and readout variables \\
\hline
\end{tabular}

Specification 1, difficult and expensive to support before low-cost, high performance computation equipment became available, is now readily met. Specification 2 evidently eliminates many well-established, conventional flow meters, amongst these, unfortunately, most of the highly accurate and reliable, listed in TABLE(I-3). Those that qualify are listed in TABLE(I-4) and further commented on in the following. 
TABLE (I-3): FM ELIMINATED BY SPEC 2

\begin{tabular}{|l|} 
Pitot tubes (single or multiple) \\
Anemometers (various types) \\
Orifice plates, Nozzles, Venturi FM \\
Rotameters \\
Turbine (Paddle-wheel) Mechanical FM \\
Target Flow meters \\
Vortex-shedding Flowmeters \\
Inserted sensing element Magmeters \\
\hline
\end{tabular}

TABLE (I-4): FM QUALIFYING UNDER SPEC 2

\begin{tabular}{|c||}
\hline (A) Composition measurement \\
Gamma (acoustic) beam transmission \\
E.M. Field \& Microwave sensing \\
Inertial mass response to a driver \\
(U-tube vibratior: \\
\hline (B) Flow velocity (Mass Flow rate) \\
Magmeters(conducting media) \\
Acoustic, Diagonal Beam \\
Acoustic-Doppler \\
Coriolis' Force Mass-FM \\
Cross Correlation (var. sensor pairs) \\
\hline
\end{tabular}

(A) Composition Measurement: Commercial Gamma beam transmission instruments of industrial type require calibration; most also need a special duct geometry (a "Z-leg") that introduces an impedance. With this proviso, they can read within 1\%; E.M. Field ("Capacitive") Densitometers likewise require calibration but are otherwise suitable for dense-slurry service. Microwave densitometry schemes are strongly sensitive to molecular structure and moisture, relatively costly and limited to ducts of modest diameter. Inertial Mass Response densitometers, requiring a U-tube, are applicable in relatively dilute, fine-consist slurries but too vulnerable to erosion or plugging for dense slurry feed lines.

(B) Flow Velocity (Mass Flow rate): Magmeters are limited to conducting (water-based) slurries and become inaccurate at the low average velocity typical of dense, highly viscous media. Acoustic Diagonal-Beam schemes, widely used for dilute slurries flowing in turbulent mode such as sewage, are inherently inaccurate unless calibrated and fail in laminar flow; Doppler schemes cannot cope with non-Newtonian flow. Coriolis' Force flow meters, a relatively recent development, are commercially available for ducts of up to 1-in diameter; attempts to design these meters for ducts of larger sizes have so far been unsuccessful. Cross-Correlation schemes seem readily applicable to a wide range of duct sizes and slurry media; the required extensive calculations can nowadays be provided without much difficulty. However, tests with a variety of sensors have so far failed to yield useable results in laminar flow; large, unprediccable errors have been found for turbulent flow. Potentially applicable or promising schemes are thus reduced to those listed in TABLE $(I-5)$.

TABLE(I-5): SEMIFINAL INSTRUMENT SELECTION

\begin{tabular}{|c|c|}
\hline $\begin{array}{c}\text { Composition. } \\
\text { (Bulk Density) }\end{array}$ & E.M. Field interrogation (Capacitive Sensing) \\
\hline $\begin{array}{c}\text { Flow Velocity } \\
\text { or }\end{array}$ & Magmeters (conducting media only) \\
Mass Flow rate & Coriolis' Force Flow meters (only small ducts) \\
\hline
\end{tabular}




\section{(C) Plan of this Report}

The present report, in preparation for several years, sets itself the general task of reviewing the measurement of flow in dense slurries, identifying specific problems and investigating these in the light of such theoretical concepts as are available - an assignment that covers a variety of topics in physics and engineering. The perspective of its authors developed in the course of several years' association with an project that was initiated at ANL under the leadership of Drs. C. Till and L. Le Sage and managed first by Dr. O'Fallon (EP Division), eventually by Dr. A.Raptis (CT Division), in response to DOE/METC-PETC. Amongst the original goals of this effort, flow measurement for coal gasification and liquefaction Pilot plants was to be provided in the form of a prototype, installable instrument. Though much information was developed, both thrcugh a helpful exchange of ideas in a series of annual Symposia(I-2) and the construction and operation of Test Loops(*), that goal was not met. Yet, the negative results obtained in the course of extensive tests were useful, if only as an indication that, rather than testing existing devices, a thorough review, based on known principles, was called for. The present report, heeding this lesson, tries to address that need.

During (and since) the time this project was active, readout functions provided by commercial instruments, taking advantage of developments in the computer industry, have become increasingly sophisticated. Aside from convenience, readout accuracy can be improved through use of formula corrections; separate instrument channels are readily combined to yield a composite readout that may be directly applied to flow and process control. While this increased capability is useful and welcome, the most significant, indeed revolutionary, result of improvements in computational speed and memory capacity is the possibilty of providing an absolute readout that combines instrument reading with fairly extensive, theory-based computations and stored information. That approach, emphasized and advocated in this report, runs counter to conventional engineering practice (which strongly relies on calibration), and thus requires justification. Chapter II reviews the arguments that seem to lead one to specify an absolute readout capability for dense slurry flow measurement. The rest of the report reviews the practical configurations and theoretical foundations of the selected schemes listed in TABLE(I-5), with the objective of producing the desired variable from a judicious combination of readout and calculations. To do this successfully, the interplay of phase and velocity distributions across the duct, determined by Fluid Mechanics, with the modus operandi, configuration and information processing routines of specific measurement schemes must be taken into account. Accordingly, a review of slurry Fluid Mechanics Basics (Chapter III) is followed detailed reviews and discussions of Sensing Scheme Basics (Chapter IV), emphasizing Gamma Transmission and E.M. Field interrogation. Commercial Instruments based on either Sensing technique are available - however, as it turns out, in configurations that do not permit the readout to be converted to the phase fraction without calibra-

(*) The ANL-SLTF(1-3) Slurry Test Loop, equipped with two unusually accurate calibration schemes: a full-flow diverter with Makeup(I-3) and an on-line Pulsed Neutron Activation scheme (briefly described in Chapter VI), and the GSTF, a gas-entrainment loop. 
tion. The material developed in Chapter IV is applied to the practical design of on-line Densitometers, including channel electronics stability enhancement schemes, crucial in the absence of frequent recalibration, in Chapter $V$.

Current industrial practice favors Velocity measurement by acoustic means (available from many instrument vendors). A fairly detailed review, provided in Chapter VI, leads one to reject these otherwise useful schemes for service on slurry feed lines and/or pipelines. A discussion of Magmeters and Coriolis' Force flow meters shows those devices to be excellent choices when inherent limitations with regard to medium conductivity or duct size allows their use. Cross Correlation as a means of velocity measurement has been studied for more than 40 years, with a variety of sensing devices: acoustic, microwave, capacitive and temperature fluctuation sensing, amongst others. The bulk of over 100 existing reports on this subject amounts to feasibility studies; however, amongst these are some measurements on test loops equipped with alternative flow speed measurement. All those reports reveal "meter factors", i.e., errors, that are both large and unpredictable. While, customarily, "meter factors" are dealt with by calibration, it has not been possible to extend the calibration range over both laminar and turbulent flow; moreover, performance in laminar flow, typical of slurry feed lines, has been too inaccurate for practical use. This failure contrasts with the accuracy of generically similar "tagging" schemes (provided that certain specific sensing and data processing methods, based on theoretical considerations, are used). A review of both tagging and Cross Correlation (CCF) points out some fallacies in the conventional treatment of CCF Flow measurement and shows that a data processing scheme based on a calculated moments ratio can eliminate those large "meter factors". The methodology supported by this review differs very considerably from current practice, adopted in the course of a long history (when Cross Correlation was a laboratory curiosity rather than a practical technique).

Chapter VII briefly reviews Systems design and combined data processing strategies; Chapter VIII summarizes and concludes this report. The somewhat complex, but important, statistics of cross-correlation processing are surveyed in Appendix A; Appendix B lists a selection of Finite-Difference programs that were used to produce the illustrations; Appendix $C$ discusses the design of a Pycnometer, as an example of the application of statistical reasoning to enhance accuracy of an instrument needed in providing supporting information for on-line gamma densitometry.

Reports on specific measurement devices too of ten stress only the good news and slight the problems. In this report, every effort has been made to present a balanced picture: judgments regarding specific devices and methods are supported by arguments based on mathematical modeling, or, if available, test results. One of the more controversial features of this report is its advocacy of Cartesian geometry for the Test Section of on-line Flow meters. This geometry is significantly superior to all circular duct-conforming electrode geometries with regard to field uniformity and duct coverage; it does not produce the "dielectric discontinuity" error that is inevitable in a wrappedplate scheme and effectively reduces slurry stratification error. All this is supported by quantitative calculations: except for diagrams whose purpose is qualitative illustration, all Flow contour, Equipotential and Field line plots presented in this report are based on solutions of the Poisson equation. 


\section{ABSOLUTE READOUT VS. CALIBRATION IN DENSE-SLURRY FLOW MEASUREMENT}

This brief Chapter sketches in some of the background details that together support the specification of an absolute readout capability, practically unheard of in industrial flow measurement in less difficult media (where calibration has long been standard practice).

Nature provides few examples of dense slurry flow; almost all such twophase media are man-made, for the specific purpose of conveying granular solids in ducts. Two types of solids conveyors are relatively common: those serving to transport mineral ores, coal etc. over long distances and those designed for in-plant, short distance conveyance. The considerable generic differences between these conveyors warrant a brief discussion.

\section{(A) Overland Slurry Transport}

Management and control of a Long-distance conveyor (II-1) does not need highly detailed information - as the composition of the medium normally does not vary a great deal, reading the local pressure and mass flowrate at suitable intervals along the pipe is sufficient(II-2). Accuracy at the $1 \%$ level and a readout fast enough to allow remedial action in case of a flow failure. Choice amongst available flowmeters is limited principally by two factors: first, the instrument must be installable on a duct of at least 12 in diameter; secondly, in view of the abrasive nature of the medium, devices must be non-intrusive, flush with the inner duct surface or external to the duct. The principal problem of long-distance slurry line operation is to keep the solids entrained, i.e., above "critical" velocity, without having to resort to costly crushing and grinding(II-3), (II-4): this requires a suitably high Reynolds' number that, in turn, mandates average velocities of at least $3 \mathrm{~m} / \mathrm{s}$ and solid concentrations of less than $30 \%$ by volume(II-2). Even above the critical velocity (discussed further on), the solids distribution within the duct will have some vertical stratification together with a higher concentration in the axial region; the extent to which these inhomogeneities affect the accuracy of the flowmeter depends in large measure on the type and construction details of the latter. This introduces yet another special consideration: initial calibration of the flowmeter, and verification of the calibration at suitable intervals, are non-trivial tasks, complicated by the large duct diameter and hostile medium. Design and construction of a long-distance slurry line usually starts with the construction of a downscaled test section or loop; if this test loop can be equipped with a full-flow diverter, it may serve the need for meter calibration. Other calibration schemes, such as injection of a radioactive tag or Pulsed Neutron Activation, are discussed further on; Pulsed Neutron Activation becomes impractical for ducts of more than 8 in diameter while radioactive tagging has other technical limitations, not to mention cost.

\section{(B) In-Plant Solids Conveyance}

In-Plant conveyance of solids by slurrying may have various purposes, depending on which flow may be turbulent or laminar, steady or intermittent. Duct diameters are expected to be smaller than for overland conveyance, The 
most important short-haul job for a slurry line is the introduction of solid reagent into a Chemical reactor or furnace. Chemical reactions making use of catalysts invariably have their best yield at high temperatures and pressures; fluidized bed combustion at elevated pressure offers a significant enhancement of energy efficiency (I-5)-(I-7). For either task, Slurry feed facilitates the introduction of the solid reagent into the pressure vessel: solids, of a fairly fine consist (as required by reaction or combustion), are mixed with a minimum of carrier fluid and passed through one or several compressor pumps. This technique has been found more effective (and environmentally benign) than belt/bucket transport and introduction into the reactor vessel through a set of lock-hoppers. Economic and energy-efficiency considerations call for keeping the fluid at a minimum, hence, solid volume fractions over 0.5 , with concomitant laminar non-Newtonian flow, are the rule.

To calibrate a Flow meter and/or Densitometer installed on such a feedline requires a Test loop on which duplicates of the on-line instruments, together with some reliable Standard device, are installed. Slurries of specific types of solid must be made up with different proportions of entraining fluid and introduced into the loop. A series of Comparison-measurements must be carried out under conditions that duplicate those at the measurement site, insofar as they have an influence on the readout. For example, one of the few available on-line densitometry schemes, E.M. Field (or "capacitive") sensing, infers the solid fraction from a reading of the bulk dielectric constant, related to the fluid and solid component dielectric constants. As reviewed in the following, the bulk dielectric constants of mixtures vary non-linearly with the intrinsic component dielectric constants and their proportions; as a general rule, dielectric constants vary with temperature. The dielectric constant of coal, in particular, varies over a wide range, generating a calibration problem even in ambient temperature and pressure conveyors since the type of coal utilized in either conversion or combustion plants rarely remains exactly the same for a reasonably long time interval. As far as flow measurement is concerned, one notes that the Reynolds' number, determining the Flow Mode, varies inversely with viscosity, another parameter that is strongly affected by temperature: for example, some slurries used in coal conversion processes only flow at all when heated.

It follows that a meaningful calibration must be carried out at and near feedline temperature, a minor research project of its own that incidentally requires a standard scheme whose readout remains reliable over the temperature range. For the task of flow measurement, a full-flow diverter with makeup flow is provided by many laboratory test loops; however none, so far as known, can handle temperatures up to $300 \mathrm{deg} \mathrm{C}$ : the design, construction and operation of such a diverter system thus constitutes yet another research project. Pulsed Neutron Activation and/or radioactive tag injection alternatives to Flow $\mathrm{Di}-$ version have been mentioned in the preceding section. Whether or not feasible, cost considerations tend to eliminate these esoteric though accurate schemes.

To sum up these observations: the cost and difficulty of calibrating instruments for feedline flow measurement offer a strong incentive for the design and implementation of an absolute-reading capability. Flowmeter specifications discussed in the foregoing are summarized in Table (II-1). 
TABLE II-1: FLOW MEASUREMENT SPECIFICATIONS FOR SLURRY LINES

\begin{tabular}{|c|c|c|}
\hline $\begin{array}{l}\text { Conveyor classification } \\
\text { Duct diameter range } \\
\text { Solid volume fraction } \\
\text { Flow mode } \\
\text { Mean velocity } \\
\text { Conveyed medium temp. } \\
\text { Pressure }\end{array}$ & $\begin{array}{l}\text { Long-Distance } \\
1-4 \mathrm{ft} . \\
<0.30 \\
\text { turbulent } \\
3-5 \mathrm{~m} / \mathrm{s} \\
\text { ambient } \\
\text { a few atm. at pump }\end{array}$ & $\begin{array}{c}\text { In-Plant } \\
1-8 \text { in } \\
>0.50 \\
\text { laminar, non-Newtonian } \\
0.6-1 \mathrm{~m} / \mathrm{s} \\
\text { ambient to }>300^{\circ} \mathrm{C} \\
\text { up to } 100 \mathrm{~atm} .\end{array}$ \\
\hline Flowmeter type & non-intrusive & non-intrusive \\
\hline Readout called for & mass flowrate & solid component feedrate \\
\hline \# of indep. measuremts. & single measurement $\mathrm{OK}$ & 2 indep. measurements \\
\hline Maximum Error & $1 \%$ OK & $<1 \%$ (both variables) \\
\hline Response time within: & $1-2 \mathrm{sec}$ & $<1 \mathrm{sec}$ \\
\hline $\begin{array}{l}\text { Principal calibration } \\
\text { problem (aside from ab- } \\
\text { rasive medium) }\end{array}$ & $\begin{array}{l}\text { Duct size: FM Cal. on Test } \\
\text { Loop needs huge Diverter; } \\
\text { no other Cal.schemes feas. }\end{array}$ & $\begin{array}{l}\text { ( } p, T \text { ) of medium precludes } \\
\text { diversion. Other methods } \\
\text { feasible but costly. }\end{array}$ \\
\hline
\end{tabular}

\section{SLURRY FLUID MECHANICS BASICS}

\section{(A) Bulk Parameters and Duct Averages}

Only few amongst many types of Flowmeters actually measure that which the readout displays, e.g., the duct-averaged flow velocity or the mass flowrate. More typically, the device response is translated to the readout. In two-phase flow of a generally non-uniform binary mixture, this involves a fair number of local and bulk parameters, whose relations are now briefly reviewed, with emphasis on the in-plant class of slurry conveyors.

Let $A=$ duct cross sectional area, da $=\operatorname{rdrd} \varphi$, circular, $d a=d x d y$, rectangular duct. Let $\rho_{s}=$ intrinsic solids density, $\rho_{f}=$ fluid density. Defining variables listed in Table (III-1) below, as well as the Discharge or Volumetric Flowrate $\mathbb{Q}$ and Mass Flowrate $M$,

TABLE III-1: VARIABLES

\begin{tabular}{|c|c|c|}
\hline variable & local & duct-average or bulk \\
\hline Solid Velocity & $V_{s}(r, \vartheta)$ & $\bar{v}_{s}$ \\
Fluid Velocity & $V_{f}(r, \vartheta)$ & $\bar{v}_{f}$ \\
Sol. Vol. Fract. & $\phi_{s}(r, \vartheta)$ & $\bar{\phi}_{s}$ \\
Density & $\rho(r, \vartheta)$ & $\bar{\rho}$ \\
\hline
\end{tabular}


we may express bulk variables in terms of certain integral ratios,

$$
\begin{aligned}
& \bar{\phi}_{g}=\int \phi_{g}(r, \phi) r d r d \varphi / A, \quad g=s, f ; \quad \text { (III-1) } \\
& \rho(r, \varphi)=\rho_{f} \phi_{f}(r, \varphi)+\rho_{s} \phi_{s}(r, \varphi) ; \\
& \bar{\rho}=\rho_{f} \bar{\phi}_{f}+\rho_{s} \bar{\phi}_{s} ; \\
& \bar{v}_{g}=\int V_{g}(r, \varphi) \phi_{g}(r, \varphi) r d r d \varphi / \bar{\phi}_{g}=\left\langle V_{g} \phi_{g}\right\rangle / \bar{\phi}_{g} ; \quad g=s, f ; \text { (III-4) } \\
& \mathbb{Q}=A\left[\bar{V}_{s} \bar{\phi}_{s}+\bar{V}_{f} \bar{\phi}_{f}\right]=\mathbb{Q}_{s}+\mathbb{Q}_{f} \\
& Q_{g}=A \bar{V}_{g} \bar{\phi}_{g} \text {, } \\
& g=s, f ; \quad(\text { III }-6) \\
& Q=A \bar{V}\left[1-\frac{\bar{V}_{f}-\bar{V}_{s}}{\bar{V}_{f}} \bar{\phi}_{s}\right] \text {, } \\
& M=\rho_{s} \mathbb{Q}_{s}+\rho_{f} \mathbb{Q}_{f}=A \bar{V}_{f}\left[\bar{\rho}-\rho_{s} \bar{\phi}_{s} \frac{\bar{V}_{f}-\bar{V}_{s}}{\bar{V}_{f}}\right] \text {. }
\end{aligned}
$$

The second term within the brackets is proportional to the velocity slip, significant for gas-entrainment but negligible in steady-state solid-liquid flow. It follows that the volumetric flowrate is closely approximated by the product of cross sectional area and duct-averaged flow velocity, the mass flowrate in turn by the volumetric flowrate - bulk density product.

Eqs. (III-2) \& (III-3) directly relate relative slurry density to the volume fractions; however, the weight fractions are usually given (typically, slurries are made up by weighing out and adding solids to a specified mixture weight). The solid weight and volume fractions $w_{s}$ and $\phi_{s}$ are connected through

$$
\rho_{\mathrm{s}} \bar{\phi}_{\mathrm{s}}=\bar{\rho} \bar{w}_{\mathrm{s}} \text {. }
$$

For an instructive example, we put $\rho_{f}=1$ (water) and let $\phi_{\mathrm{s}}$ vary from 0 to 0.6 , whilst $\rho_{s}$ is chosen between 1 and 6 ; the resultant $w_{s}$, plotted in FIG (III-1), portrays the strongly non-linear relation between these fractions.

Eqs. (III-1)-(III-8) do not exclude the possible presence of a third phase (gas). Entrained air is of ten present in dilute slurries but tends to be minimal for dense slurries, especially at higher pressures. We thus suppose, for present purposes, that anywhere in the duct or on he average,

$$
\phi_{s}(r, \varphi)+\phi_{f}(r, \varphi)=1
$$




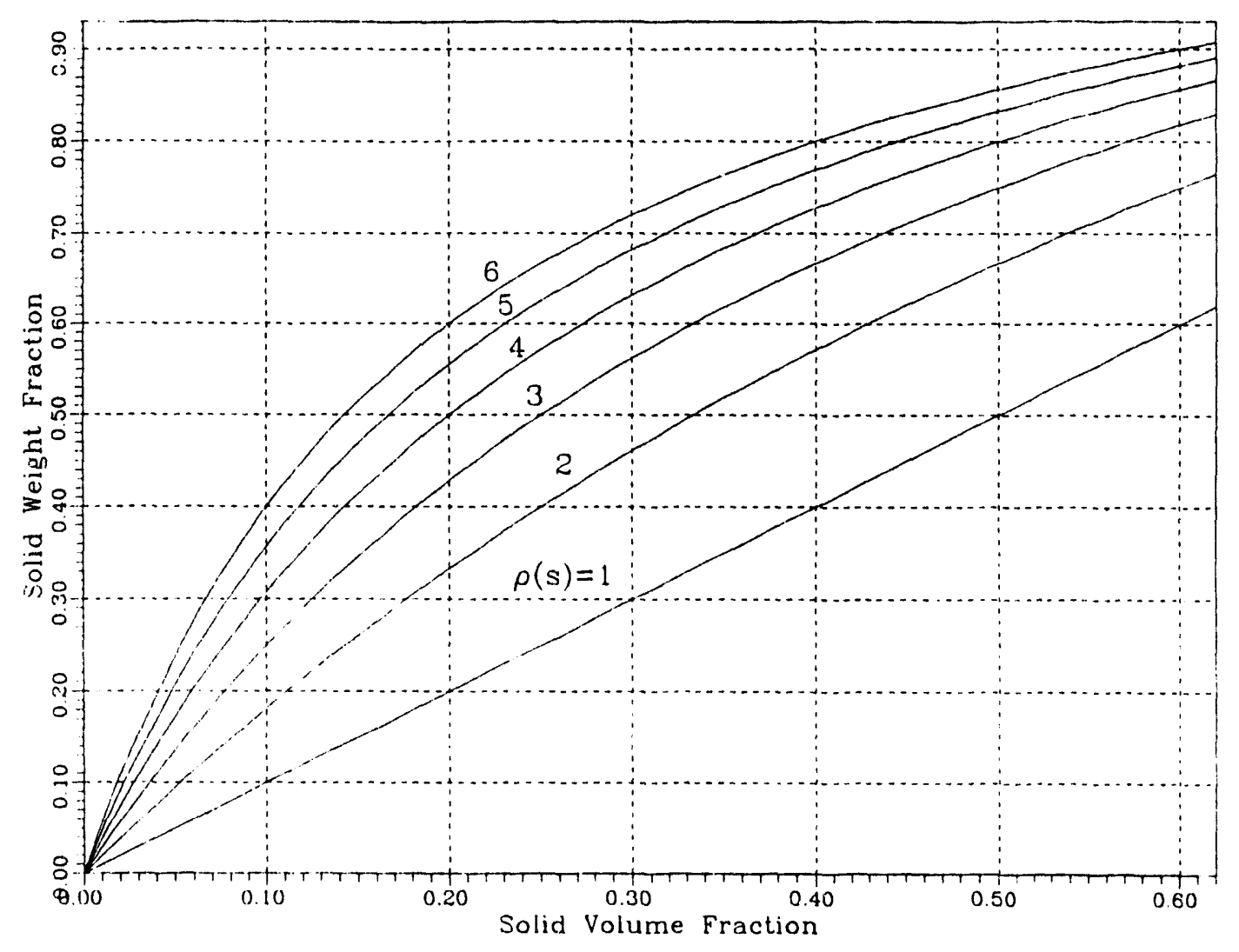

(FIG (III-1): Volume and Weight Fraction

A variable that is sometimes specified for mixed flow is the "Loading" or solid to fluid discharge ratio

$$
\mathbb{L}=\frac{M_{s}}{M_{f}}=\frac{w_{s}}{1-w_{s}} .
$$

The foregoing simple relations indicate that measurements of $\bar{\phi}$ and $\bar{V}$ suffice to determine the solid feedrate $\mathbb{Q}_{\mathbf{s}}$. Instead of a velocity measurement, available from a Magmeter or Cross-Correlation scheme, one may consider, e.g., a Coriolis' force Flow meter measurement of $M$. The former only requires the duct area $A$, the latter, the intrinsic density of either phase:

$$
\mathbb{Q}_{s}=M \bar{\phi}_{s} /\left[\rho_{f}+\left(\rho_{s}-\rho_{f}\right) \bar{\phi}_{s}\right]=M w_{s} / \rho_{s}
$$

\section{(B) Fluid Mechanics of Entrained Solids Flow}

The Theory of Fluid Mechanics relies on a set of five Equations: of State, Constitutive (Rheological), Mass, Momentum and Energy Balance; for two phases, the number of Equations is doubled. However, a solution that allows the pre- 
diction of variables is available only for elementary cases. To make any progress at all under ordinary circumstances, additional assumptions must be introduced; mathematical formulae resulting from these assumptions of ten contain one or more constants whose values must be sought experimentally. The next section presents an elementary case, the determination of the velocity map for laminar flow in a rectangular duct from solutions of the momentum (NavierStokes) equation produced by Finite-Difference. To yield solutions for Pseudoplastic flow, the additional assumption of a "power law" two-parameter rheological equation is introduced. How laminar (Newtonian and non-Newtonian) flow fits into the general scheme is shown schematically in the Flow Mode TABLE (III-1). The Flow Modes listed in the Table break down into Laminar and Turbulent as well as Newtonian and Non-Newtonian, the latter including Pseudoplas-

TABLE (III-2): FLOW MODES

\begin{tabular}{|c|c|c|}
\hline QUASI-HOMOG. & INHOMOGENEOUS & STRATIFIED \\
\hline NEW-LAM & NEW-TURB & \\
\hline PSEU-LAM & PSEU-TURB & \\
\hline BING-LAM * & BING-TURB * & \\
\hline DILLAM & DL-TURB : & \\
\hline THX-LAM 8 & THX-TURB & \\
\hline RHE-LAM & RHE-TURB 2 & \\
\hline
\end{tabular}

$\begin{array}{lll}\text { NEW-LAM : Newtonian-Laminar; } & \text { NEW-TURB : Newtonian-Turbulent } \\ \text { PSEU-LAM : Pseudoplastic-Lam.; } & \text { PSEU-TURB : Pseud.-Transition/Turbulent } \\ \text { BING-LAM : Bingham-Laminar; } & \text { BING-TURB : Bingham-Trans./Turbulent } \\ \text { DIL-LAM : Dilating-Laminar; } & \text { DIL-TURB : Dilating-Trans./Turbulent } \\ \text { THX-LAM : Thixotropic-Laminar; } & \text { THX-TURB : Thixotropic-Trans./Turb. } \\ \text { RHE-LAM : Rheoplastic-Laminar; } & \text { RHE-TURB : Rheoplastic-Trans./Turb. }\end{array}$

tic, Bingham, Dilating. Thixotropic and Rheoplastic. The three columns of this Table distinguish the flowing medium as Quasi-Homogeneous(characterizing suspensions of very small particles), Inhomogeneous (larger or heavier particlos that cannot be kept in suspension by Brownian motion but will tend to assume axially symmetric distributions when suspended by vigorous turbulence) and Stratified (typical for still coarser consists). Only quasi-homogeneous media can flow in the diverse laminar modes indicated in the first column, or alternatively, at higher particle-Reynolds' numbers, in the Turbulent modes indicated in the second column. Stratified flow may develop in pipelines; modes marked with an @ symbol are only rarely encountered in slurry conveyance and not covered in this report. Bingham media (marked with an asterisk), somewhat 
less common than Pseudoplastic media, have also been omitted and may be dealt with in a future report.

In designing a slurry conveyor or instrument for a set of specifications (duct size, liquid, solid, particle size, solid concentration and flow speed), it would certainly be useful to know the Flow mode; however, theory still cannot predict this, nor furnish more detailed parameters such as the Bingham yield stress and/or Pseudoplastic Flow Behaviour Index. This information must thus be sought by measurement, a task that requires special equipment when the medium is normally conveyed at high temperature and pressure (and may not flow at all at ambient temperature).

\section{(C) Velocity Map for laminar non-Newtonian Flow in a Square Duct}

\section{(1) Introduction}

Chapter I pointed out the need for sensing uniformity in either density or velocity measurement; in Chapter IV, it will be noted that EM Field sensing varies with the square of the electric field strength. Inasmuch as uniform fields, covering the entire duct cross section, can be provided only for rectangular or square ducts, EM Field sensing accuracy is evidently best when a rectangular measurement section can be provided. As discussed below, this allows a straightforward correction for solids settling in pipeline conveyors while enabling the accurate calculation of the solids discharge in turbulent flow, from a weighted integral ratio based on Cross Correlation.

For laminar flow, a different strategy applies. To develop this, we first note that, whatever the velocity contour pattern happens to be, there exists a contour along which the local velocity just equals the duct-average velocity. We then further note that, if one models Pseudoplastic flow by a power law, this contour remains effectively unchanged over a wide range of flow modes(laminar Newtonian/Pseudoplastic). It follows that if means can be found to limit sensing to a narrow region bracketing this contour, then the Cross-Correlation Function will read the average velocity rather than the entire transit-time distribution. In order to explore this possibility further, we shall need a velocity contour calculation for Newtonian as well as Power-law flow within a square duct.

Newtonian flow through ducts of non-circular cross section was treated previously by Cornish(SQ-1) and by Lea and Tadros(SQ-2). Non-Newtonian flow has been more recently dealt with, as discussed in refs. (SQ-3)-(SQ-6). Kosicki(SQ-3), applying certain general relations between bulk parameters to a variety of flow mode formulae and duct profiles, produced a set of general predictive equations that contain "shape factors" for specific duct geometries; further detail is provided in a review article by Wohl (SQ-4). However, these equations, useful though they indubitably are to the design engineer, do not readily lend themselves to the prediction of detailed velocity contours. The treatment by Schecter (SQ-5), making use of a Variational principle, likewise is difficult to apply directly to the task we set ourselves. Wheeler and Wissler (SQ-6) employ Overrelaxation (SQ-7), (SQ-8) in connection with FiniteDifference modeling (SQ-9), (SQ-10), a technique that appears readily adaptable and thus was used in the work described in this report. 


\section{(2) Calculations}

The point of departure for these calculations is the Navier-Stokes equation, governing flow of any fluid in any mode (SQ-11). The equation is cast in Finite-Difference terms; a first approximation is provided and refined through interactive iteration until the error becomes smaller than a certain acceptable limit. In the calculations under discussion, a 64 by 64 point grid, covering the square duct, was used to produce a velocity map; a set of constant velocity increment contours was then calculated by interpolation between mesh points; in addition, a contour of special interest, that of the duct-average velocity $\langle V\rangle$, was calculated.

The Navier-Stokes equation for incompressible, viscous media features a constant term that is not known a priori and thus requires interactive adjustment. Boundary conditions include: zero velocity at the walls, maximum velocity at the center, normalized duct-average $=1$, reflection symmetry at diagonal planes and planes through center of each side. For Power-law flow (SQ12), the Flow Behaviour Index is specified.

In the following, the general incompressible,viscous fluid Navier-Stokes equation (discussed in Fluid Mechanics texts such as ref. (SQ-11) is first used to treat Newtonian (constant viscosity) flow, for which the equation becomes elementary and can be readily cast into Finite Element terms. The programmatic solution of that equation by iterative smoothing is briefly discussed. Next, the form of the equation that applies to Power-Law (viscosity proportional to local velocity gradient) flow is expressed in Finite Element terms and its solution discussed. Illustrating these calculations, Contour maps and other calculational results are provided.

Let

$$
\begin{aligned}
X, Y= & \text { coordinates, } \\
a= & 1 / 2 \text { sidelength, } \\
\mu= & \text { viscosity }(\mathrm{gm} / \mathrm{cm} / \mathrm{sec}), \\
V(X, Y)= & \text { velocity (in the axial direction) at point } X, Y, \\
\Delta p= & \text { pressure difference at two planes normal to duct axis, separa- } \\
& \text { ted by distance } \Delta z, \\
\tau_{w}= & \text { shear stress at the duct wall. }
\end{aligned}
$$

The Navier-Stokes equation for unidirectional, laminar flow of an incompressible medium reads

$$
\frac{\partial}{\partial X}\left(\frac{\mu \partial V}{\partial X}\right)+\frac{\partial}{\partial Y}\left(\frac{\mu \partial V}{\partial Y}\right)+\frac{\Delta p}{\Delta Z}=0
$$

Equating the energy loss due to wall friction, $S \Delta Z^{2} \tau_{w}$ for constant wall shear-stress, to the loss of internal (molecular motion) energy $A \Delta Z \Delta p$, 


$$
\begin{gathered}
\tau_{w}=(A / S) \Delta p, \\
\Delta p / \Delta Z=(S / A) \tau_{w}=(2 / a) \tau_{w} \\
A=\text { cross sectional area }=4 a^{2}, \\
S=\text { circumference }=8 a .
\end{gathered}
$$

The functional form of $\tau_{w}$ is determined by the flow mode, as discussed below.

\section{(a) Velocity Map for Newtonian Flow}

"Newtonian" flow is characterized by the relation

$$
\tau_{w}=-\mu(d V / d n)_{w}
$$

where differentiation is in the direction normal to the velocity contour; substituting Eq (4) in Eq (1),

$$
\nabla^{2} V-(2 / a)(d V / d n)_{w}=0
$$

It is convenient to normalize coordinates and velocities,

$$
x=X / 2 a, \quad y=Y / 2 a, \quad v=V /\langle V\rangle
$$

whence one obtains

$$
\nabla^{\prime 2} v-4\left(d v / d n^{\prime}\right)_{w}=0
$$

$(\nabla$ ' referring to the dimensionless coordinates $x, y$ ). To solve Eq. (III-19), a Finite-Difference mesh is specified; initial velocities at mesh points are either estimated from an approximation formula or produced by repeated division. Estimates of the constant term $4\left(\mathrm{dv} / \mathrm{dn}^{\prime}\right)_{w}$ must also be provided.

Based on a uniform mesh $\Delta X=\Delta Y=2 a / N, N=64$, say, $\Delta x=\Delta y=\Delta=1 / 64$, the Finite-Element version of $\mathrm{Eq}$ (II-14) comes to (in two alternate formulations),

$$
\begin{aligned}
& {\left[v_{1+1,1}+v_{1-1, J}+v_{1, j+1}+v_{1, j-1}-4 v_{1, j}\right]-4 \Delta v_{w} \Delta=0 \quad \text { (I I I-20a) }} \\
& {\left[\sum v_{k}-4 v_{0}\right]-4 \Delta\left(\Delta v_{w}\right)=0} \\
& \sum v_{k}=v_{N}+v_{S}+v_{E}+v_{W} \text {. }
\end{aligned}
$$

Subscripts $k=N, S, E, W$ denote points North, South East \& West of point 0. For $\Delta=1 / 64$, we put 


$$
-4 \Delta\left(\Delta v_{w}\right)=-\Delta v_{w} / 16=Q
$$

and note that the velocity gradient outward from the wall is negative, hence $Q$ is positive. Rearranging Eq(II-2Ob), one obtains the $\mathrm{N}-\mathrm{S}$ iteration formula

$$
v_{0}=\left[\sum v_{k}+Q\right] / 4
$$

Inherent symmetries of the geometry allow one to limit calculation to one quadrant and fill in the other quadrants by copying. As a reasonable model for calculation of first-estimate mesh point velocities, the formula

$$
v(x, y)=16 v(a, a) x y(1-x)(1-y)
$$

was chosen, in analogy to flow in a circular duct or slot. For the centerpoint velocity $v(a, a)$, a first-estimate value intermediate to these two cases,

$$
v(a, a)=2 \cdot 10
$$

was specified; similarly, the wall velocity gradient $\Delta v_{w}=-2 v(a, a)$ was adopted. Initial values are adjusted by iteration, based on successive applications of the iteration formula (II-20c) to adjacent cells, one freshly corrected value thus being used in the following cell calculation. To avoid bias, iteration runs in an up-down, left-right "Boustrophedon" pattern until the entire quadrant has been covered; the process is repeated a number of times. Convergence is judged from a monitor display of sample values, provided upon completion of each pass; a stop option terminates the calculation when successive passes agree within some desired margin. Fine tuning of the $\Delta v$ estimate is provided to speed convergence; computation of the overall cell average and comparison with unity furnishes the adjustment criterion (in practice, 16-20) passes were found to reduce the error to less than e $(-5))$.

\section{(b) Velocity Map for Power-law (Pseudoplastic) Flow}

For Power-law flow, Eq (III-17) must be replaced by

$$
\tau_{w}=-\mathrm{K}(\mathrm{d} V / \mathrm{d} n)^{\mathrm{m}}=-\mu(\mathrm{d} V / \mathrm{d} n)
$$

the parameter $\mu$ is here defined as

$$
\mu=K(d V / d n)^{m-1} \text {. }
$$

Thus, the first term of the $\mathrm{N}-\mathrm{S}$ equation may be written

$$
\frac{\partial}{\partial X}\left(\frac{\mu \partial V}{\partial X}\right)=K \frac{\partial}{\partial X}\left(\frac{F \partial V}{\partial X}\right)=K\left[F \frac{\partial^{2} V}{\partial X^{2}}+\frac{\partial F}{\partial X} \frac{\partial V}{\partial X}\right]
$$

in terms of a function $F$ defined by 


$$
F=\left[\left(\frac{\partial V}{\partial X}\right)^{2}+\left(\frac{\partial V}{\partial Y}\right)^{2}\right]^{(m-1) / 2}
$$

Expanding,

$$
(\partial F / \partial X)(\partial V / \partial X)=\left[\partial^{2}(F V) / \partial X^{2}-F \partial^{2} V / \partial X^{2}-V \partial^{2} F / \partial X^{2}\right] / 2,
$$

hence Eq (1) can be expressed entirely in terms of second differentials:

$$
\nabla^{2}(F V)+F \nabla^{2} V-V \nabla^{2} F+(4 / a K) \tau_{w}=0
$$

Normalizing as in the foregoing to $\langle V\rangle$ and $2 a$, and introducing

$$
\begin{gathered}
f=\left[(d v / d x)^{2}+(d v / d y)^{2}\right]^{(m-1) / 2}, \quad F=[\langle v\rangle / 2 a]^{m-1} f, \\
\nabla^{\prime 2}(f v)+f \nabla^{\prime 2} v-v \nabla^{\prime 2} f-8\left(d v w_{w} / d n\right)=0 .
\end{gathered}
$$

The finite-element formulation of $\nabla^{2} u$ is given by Eq. (III-21); the function $f$ becomes

$$
f(i, j)=\left[\left[v_{i+1, j}-v_{1-1, j}\right]^{2}+\left[v_{1, j+1}-v_{1, j-1}\right]^{2}\right]^{(m-1) / 2} /(2 \Delta)^{m-1}
$$

or, alternatively,

$$
f_{0}=\left[\left(v_{N}-v_{S}\right)^{2}+\left(v_{E}-v_{W}\right)^{2}\right]^{(m-1) / 2} /(2 \Delta)^{m-1}
$$

Inserting this in Eq (12') and rearranging, one obtains, explicitly,

$$
\begin{aligned}
& \sum_{k}\left(v_{k}-v_{0}\right)+f_{0} \sum\left(v_{k}-v_{0}\right)+P=0 ; \\
& P=-\left[2 \Delta v_{w}\right]^{m} / 16=(32 Q)^{m} / 16 .
\end{aligned}
$$

Eq (III-27) connects the 13 point velocities, increasing from $W$ to $E$ and $S$ to $N$, shown in the diagram below:

$$
\begin{array}{ccccc} 
& v_{\text {NN }} & \\
v_{\text {WW }} & v_{\text {NW }} & v_{N} & v_{\text {NE }} & \\
& v_{0} & v_{E} & v_{\text {EE }} \\
v_{\text {SW }} & v_{S} & v_{\text {SE }} & \\
& & v_{\text {SS }} &
\end{array}
$$


Note that $f_{0}, f_{k} \Rightarrow 1$ as $m \Rightarrow 1$, whence, according to Eq. (III-27),

$$
2 \sum\left(v_{k}-v_{0}\right)-2 \Delta v_{w} / 16 \Rightarrow 0,
$$

as expected (cf. Eq. (III-21c)). Let now

$$
\begin{aligned}
& A_{N E}=\left[F_{E}\left[V_{E}-V_{0}\right]+F_{N}\left[V_{N}-V_{0}\right]\right], \\
& A_{S W}=\left[F_{w}\left[V_{0}-V_{w}\right]+F_{s}\left[V_{0}-V_{s}\right]\right], \\
& B_{N E}=\left[\left(V_{E}-V_{0}\right)+\left(V_{D}-V_{0}\right)\right], \\
& B_{S W}=\left[\left(V_{0}-V_{w}\right)+\left(V_{0}-V_{s}\right)\right],
\end{aligned}
$$

then Eq. (III-27) simplifies to

while

$$
A_{\mathrm{NE}}-A_{\mathrm{SW}}+\mathrm{F}_{\mathrm{O}}\left[\mathrm{B}_{\mathrm{NE}}-\mathrm{B}_{\mathrm{SW}}\right]+P=0 \quad([11-28)
$$

$$
\begin{array}{lll}
F_{D}=\left[\left[V_{N}-V_{S}\right]^{2}+\left[V_{E}-V_{W}\right]^{2}\right]^{(m-1) / 2}, & (I I I-29 a) \\
F_{E}=\left[\left[V_{E E}-V_{0}\right]^{2}+\left[V_{N E}-V_{S E}\right]^{2}\right]^{(m-1) / 2}, & (I[I-29 b) \\
F_{S}=\left[\left[V_{O}-V_{S S}\right]^{2}+\left[V_{S E}-V_{S W}\right]^{2}\right]^{(m-1) / 2}, & (I I I-29 C) \\
F_{N}=\left[\left[V_{N N}-V_{D}\right]^{2}+\left[V_{N E}-V_{N W}\right]^{2}\right]^{(m-1) / 2}, & (I I[-29 d) \\
F_{W}=\left[\left[V_{0}-V_{W W}\right]^{2}+\left[V_{N W}-V_{S W}\right]^{2}\right]^{(m-1) / 2} . & (I I I-29 e)
\end{array}
$$

(c) Additional Rows \& Columns needed for l teration

Wall-to-wall iteration requires one additional row (outside the bottom/ left duct wall) and three rows/columns beyond the centerpoint (top/right edge of quadrant). This calculation is straightforward in Newtonian (initial) approximation but more complex for Power-law flow; detailed calculations are given below. Rows/columns are relabeled: duct wall at $(\mathrm{I}, 2)$; $(2, \mathrm{~J})$; first iterated velocity $=\mathrm{V}(3,3)$; last $=\mathrm{V}(\mathrm{N}+2, \mathrm{~N}+2)$. After adding the indicated points, $F_{k}$ and $A_{k j}$ are calculated; then the iteration form of Eq (II-27),

$$
V_{0}=\left[A_{N E}-A_{S w}+F_{0}\left(V_{N}+V_{S}+V_{E}+V_{w}\right)+P\right] / 4 \quad(I I I-30)
$$

or,

$$
\begin{aligned}
V(I, J) & =[F(I, J+1)[V(I, J+1-V(I, J)]+F(I+1, J)[V(I+1, J)-V(I, J)] \\
& -F(I, J-1)[V(I, J)-V(I, J-1)]-F(I-1, J)[V(I, J)-V(I-1, J)] \\
& +F(I, J)[V(I, J+1)+V(I, J-1)+V(I+1, J)+V([-1, J)]+P] / 4\left(I I I-30^{\prime}\right)
\end{aligned}
$$

is applied to succesive points $I=3, \ldots, N+2 ; J=3, \ldots, N+2$.

Passes are repeated a number of times; thereupon, the average is calcula- 
ted and compared with unity: if needed, the constant $P$ is adjusted; if not, the array is recorded. The set of velocity map calculations starts with the Newtonian grid $(m=1)$ and proceeds to $m=0.9$; that result becomes the initial set for $m=0.8$ etc. to $m=0.5$.

\section{(d) Velocity Contours}

To obtain a set of, say, $N$ velocity contours, the centerflow velocity is divided by $N$, whereupon the contour is interpolated from $x / a=0$ to the point where the slope exceeds 45 deg; the remaining points can then be copied and stored in memory as a set of $(x, y)$ arrays that can be read in by the plotting program. FIGS (III-2a)-(III-2d) present a sample of four such plots for Metzner Indices ranging from unity (Newtonian flow) to 0.4, covering the range of medium viscosities one would expect to find in slurry feedlines. The plots clearly indicate the increasingly flat slope near the center and steep slope near the wall that characterizes the Pseudoplastic Mode.

Of special interest to this investigation are those velocity contours where the local velocity just equals the duct-average, designated here, for convenience of referral, as the "Specific" contour for a given flow mode. A plot of these contours, presented in FIG (III-3), reveals the perhaps surprising, but potentially useful, fact that Specific Contours remain virtually fixed over a wide range of flow modes(*), whose flow velocity pattern, on the other hand, changes sufficiently (cf. FIGS III-2) to introduce serious errors in the readout of flowmeters sensitive to this pattern.

\section{SENSING TECHNIQUE BASICS}

\section{(A) Introduction}

From the preceding review of Fluid Mechanics that apply to dense slurries, we turn now to basic information on selected non-intrusive techniques for sensing a two-phase medium flowing inside a duct. The term "basic" is taken here to mean that this information is required for an error-free readout of either, or both, phase fraction (bulk density) and duct-averaged velocity. As considered in the foregoing, the conventional approach, calibration against another, presumably reliable device or instrument is not a practical option in some of the scenarios this report emphasizes. Instead, basic information, describing the interaction of the interrogating agent with the local environment in quantitative terms, must be utilized to interpret sensor signals. This requires a theoretical framework that permits the extraction of desired variables from the readout, a reference readout (for which the duct is filled with the carrier liquid), physical constants, tabulated data and laboratory measurements that can be provided even in a difficult on-line scenario (e.g., Chemical composition of both phases). In turn, this extraction requires unbiased sensing of the duct interior, implying means of directing the sensing device so as to "view" only the medium within the duct.

(") this behaviour is of course also found in circular ducts. 


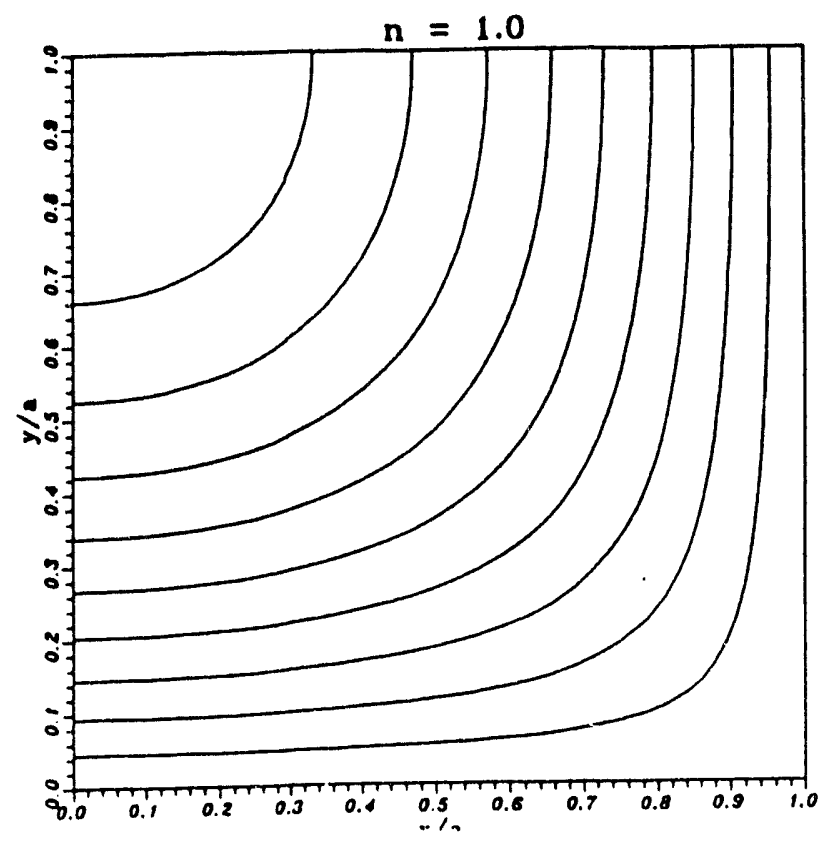

FIG(III-2a): Velocity Contours, Square Duct, Newtonian-Laminar Flow $(n=1.0)$

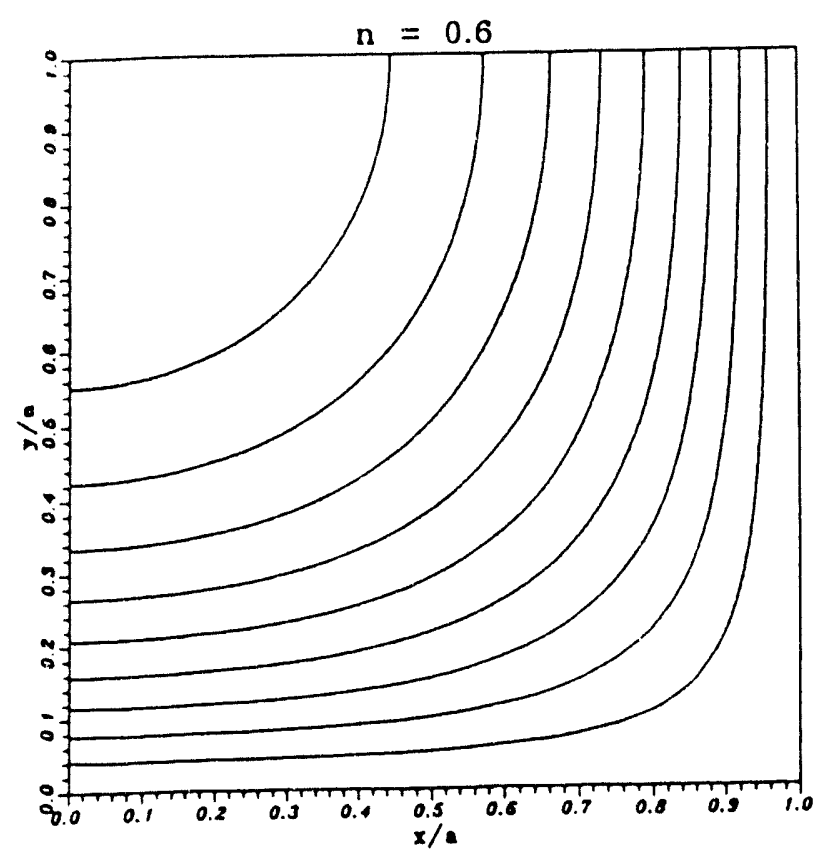

FIG(III-2c): Velocity Contours, Square Duct, Pseudoplastic Flow $(n=0.6)$

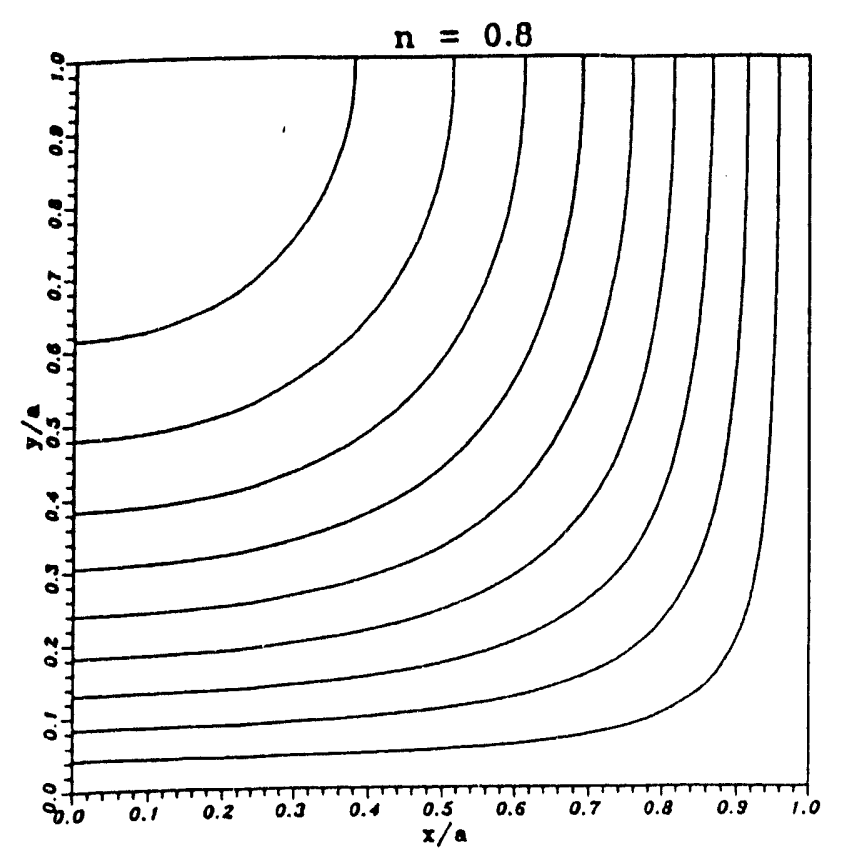

FIG(III-2b): Velocity Contours, Square Duct, Pseudoplastic Flow $(n=0.8)$

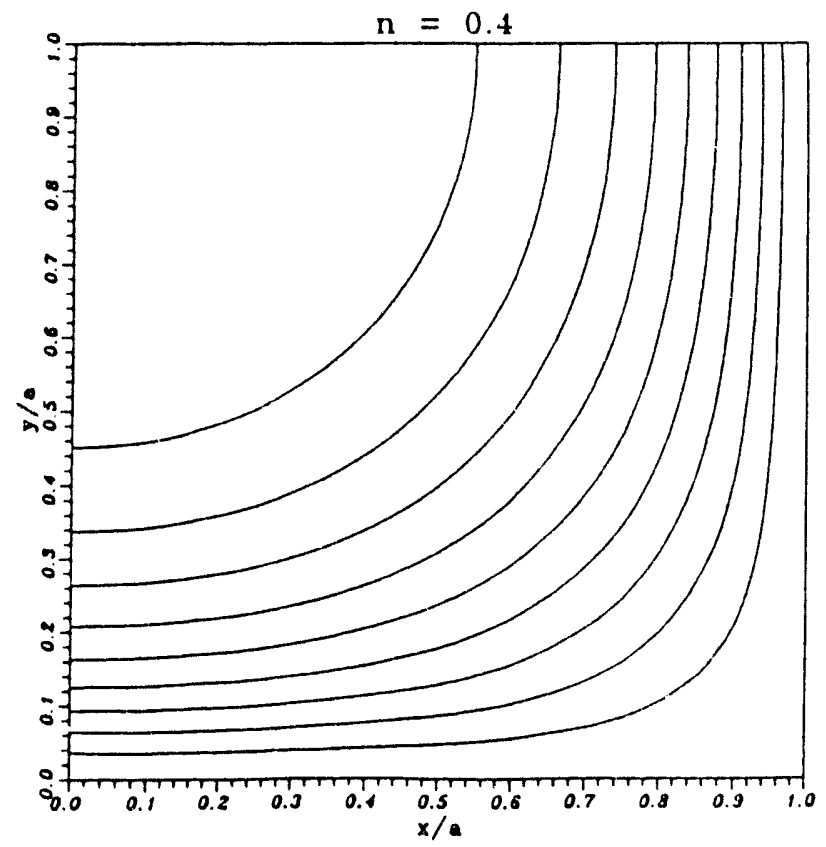

FIG(III-2d): Velocity Contours, Square Duct, Pseudoplastic Flow $(n=0.4)$ 


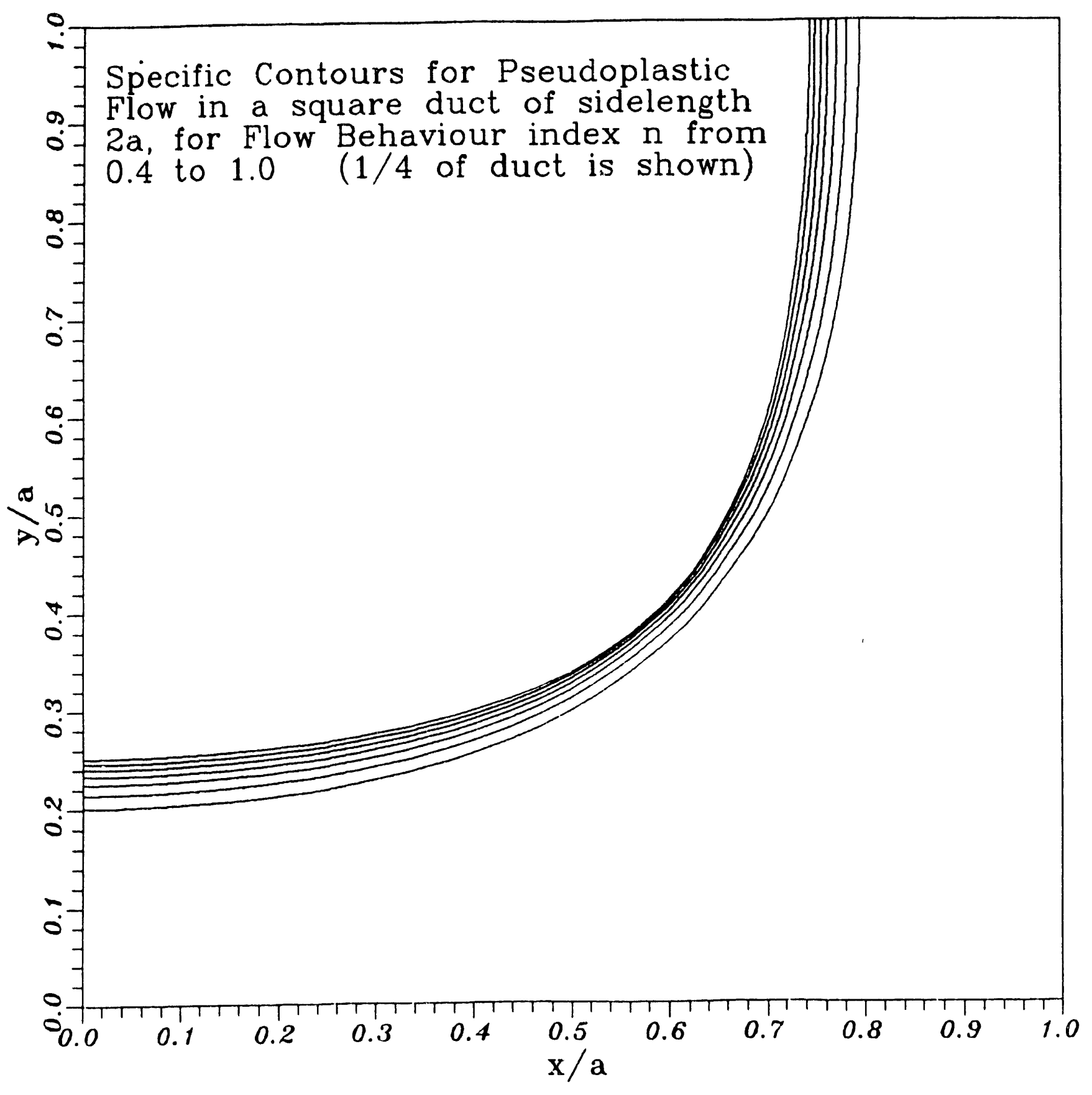

FIG (III-3): "Specific" Velocity Contours, $\mathrm{n}=0.4$ to 1.0 
Sensor direction is not emphasized in "Gamma-meters" offered by commercial vendors for Industrial on-line installation, and ignored altogether in many EM Field interrogation schemes, described in Journal reports and the $\mathrm{Pa}-$ tent literature. Available by elementary means for Gamma Transmission, direction constitutes the central problem of EM sensing electrode geometry design a problem that has not found a satisfactory solution and thus calls for a detailed discussion. With regard to interaction, both Gamma Transmission and EM sensing require a review of formulae that interpret the readout signal in terms of the phase makeup. Each scheme further involves certain errors that can be either corrected for or dealt with (as is the case for fluctuation error, affecting both) through appropriate data acquisition and processing schemes.

Acoustic on-line interrogation, non-intrusive, convenient, inexpensive and commercially available from many vendors, is omitted in this review. There are two reasons for this: first, a theoretical framework that would allow the interpretation of sound wave attenuation in terms of interaction with randomly arranged particles is not available, secondly, no means are known that would make existing acoustic schemes sense different parts of the duct interior with sufficient uniformity. This appears to make Acoustic sensing, while indubitably useful in general, unsuited in the particular case of a dense slurry medium. Gamma Transmission is also considered here as unsuited to CrossCorrelation velocity sensing: Like acoustic beam interrogation, its sensing uniformity is strongly biased; moreover, as Cross-Correlation is severely impacted by the Poisson statistics of the transmitted gamma count rate, it must be ruled out on statistical grounds, leaving EM Field sensing as the method of choice for Cross-Correlation velocity measurement. For Phase fraction determination, Gamma Transmission is inherently more accurate, but less compatible with an Industrial environment, than EM sensing. With hardware designed for clamp-on service, Gamma Transmission could thus serve to calibrate an on-1ine EM sensing densitometer installation.

\section{(B) Gamma Transmission}

\section{(1) Introduction.}

The interaction of gamma radiation with bulk media is completely determined by the interaction probability of individual photons, varying with photon energy and medium atomic (Chemical) composition, and proportional to the path length. The energy dependence of photon interaction cross sections is based on theoretical formulations developed in the early thirties (G-1), (G-2). Cross section Tables, calculated on the basis of this theoretical framework and accurate to about $1 \%(G-3),(G-4)$, allow the quantitative prediction of photon removal from a collimated beam that traverses a sample of known elemental composition. It follows further that a complete, quantitative assay of a $\mathrm{N}-$ element medium can be provided by transmission measurements with $\mathrm{N}-1$ sources of different energies. Likewise, given M Components (e.g., Ash, Fixed Carbon and Moisture for coal samples) whose atomic composition has been predetermined, M-1 gamma sources of different energies allow a determination of the sample Component makeup(G-5)-(G-7). 
To make such an assay accurate, registration of secondary photons, scattered or generated in the medium or elsewhere, must be prevented by a judicious combination of "Good-Geometry" collimation and pulse-height selection, calling for pulse counting rather than current electronics. While this type of system has long been employed in laboratory densitometry and composition measurements, industrial on-line gamma densitometers, trading off performance against cost and resistance to damage, tend to use less expensive and more rugged photon detectors in current reading mode, incapable of discrimination against secondary radiation, with collimation (entailing additional cost and weight) kept to a minimum $(\mathbf{G}-8)-(G-10)$. Photon interaction theory can only roughly approximate the response of this type of equipment, which therefore must be calibrated (in contrast, equipment designed as specified above can calibrate the response of other densitometers). For a scenario that effectively precludes on-line or test loop calibration, a carefully designed pulse-counting, Good-Geometry scheme, even though more costly, is evidently preferable to a conventional current-reading gamma densitometer of commercial type. Potential savings through improved process control and energy-efficiency must be weighed against $\cos ^{+}$

To produce an absolute readout, as advocated here, it suffices to know:

(A) the intrinsic density of the solid, requiring careful sampling and laboratory measurement at the plant;

(B) Hydrogen weight fractions of both phases;

(C) weight fractions of all other major constituents of both phases.

For coal, the Hydrogen weight fraction is available through ASME-specified "Ultimate" analyses (on the basis of which coal is traded). A sufficiently accurate estimate of other major constituents can be obtained from "Proximate" analyses and a "Standard Ash" model (Elemental Analysis of samples, requiring the services or use of a suitably equippped and staffed laboratory, can be useful for occasional verification). That a full-fledged Elemental Analysis is not needed for routine low-Z medium densitometry is due to certain regularities in the cross sections and atomic weight/number ratio. This peculiarity, discussed in the following, makes gamma transmission especially useful in the solid feedrate measurement of coal slurries.

For on-line measurement of a medium with $N=2$ phases (such as a slurry substantially free from entrained gas), a single monoenergetic gamma source suffices. The mathematical relations between phase chemical composition, phase fraction, transmitted count and reference count (in this case with pure fluid present in the duct) are presented in the next section, followed by a discussion of data processing options and several types of error corrections.

Comparing Gamma transmission and other non-intrusive composition measurement schemes on a technical basis, we note that gamma beam interrogation requires a reasonably high density contrast between the phases and thus becomes difficult to manage when that contrast is weak. E.M.Field interrogation, the other scheme this report singles out, senses dielectric contrast (which may be strong when density contrast is weak, or, vice versa). Specific advantages offered by gamma transmission in general (and exploited by the system described here in particular) include: 
- Insensitivity to particle shape, size and size distribution

- Negligible effect on readout by environment (temperature, pressure)

- Insensitivity to non-uniform phase distributions along the beam

- Weak sensitivity to non-uniform phase distribution across the beam

- Insensitivity to molecular structure, Chemical state of medium

* 2-source reading capability of 3-phase media (aerated slurries)

Temperature, pressure and molecular structure affect E.M., Microwave and NMR sensing; acoustic transmission is particularly sensitive to particle size and size distribution. Gamma Transmission drawbacks include:

* High cost of sensing equipment, electronics and maintenance required

* Susceptibility to damage

* Weight of needed Shielding/Collimation, requiring special support

- Radiation Hazard, requiring licensing, personnel monitoring etc.

\section{(2) Gamma Transmission: Homogeneous, Uniform Medium}

To introduce the equations that allow inference of the phase fraction within a two-phase medium from a transmission measurement, we consider, as a preliminary exercise, a well-collimated beam, impinging on a homogeneous, uniform medium of thickness $x$ within a container and intercepted at the rear of the container by a photon detector. Let

$C=$ count accumulated over a certain time interval $t$,

$\mathrm{C}_{\mathrm{o}}=$ comparison count for time $t$, with medium removed,

$\sigma_{z}=$ Photon removal cross section for atom number $Z$, in barn/atom units,

$\mathrm{n}_{\mathrm{z}}=$ number density of atoms $Z, \mathrm{n}_{\mathrm{z}}=\left(N \rho \omega_{\mathrm{z}}\right) / \mathrm{A}_{\mathrm{z}}$,

$\mathrm{A}_{z}=$ atomic weight; $\omega_{z}=$ element weight fraction,

$N=0.6022=$ Avogadro's number for $\sigma$ in barn/atom units,

$\rho=$ bulk density of the medium,

then,

$$
C=C_{0} \exp \left(-x \sum n_{z} \sigma_{z}\right)
$$

The photon removal cross sections $\sigma$ are sums of cross sections for four main gamma photon interactions, each varying in a specific manner with photon energy as well as with a different power of the atomic number $Z$. In principle, this could make the calculation of photon removal ambiguous. However, for .1 ight elements $Z<50$ and photon energies $200 \mathrm{keV}<E<5 \mathrm{MeV}$, Compton scattering strongly dominates over other interactions (G-4). The Compton cross section is closely proportional to the first power of $Z$, allowing the removal cross section to be expressed in terms of the Hydrogen cross section,

$$
\begin{gathered}
\sigma_{z}=Z \sigma_{H}\left(1+\beta_{z}\right) ; \quad \beta_{z} \ll 1 ; \\
\sigma_{H}=0.256 \text { barn/atom for } 611 \mathrm{keV}{ }^{137} \mathrm{Cs} \text { radiation. }
\end{gathered}
$$


Selected correction factors $\beta$ at $611 \mathrm{keV}$, calculated from ref.(G-4), are shown in FIG $(I V-1)$ and 1 isted in Table IV -1 .

Again for lighter elements, $2 / A$ can be approximated by 0.5 ; thus

$$
2 / A_{z}=0.5\left(1-\alpha_{z}\right) ; \quad \alpha_{z} \ll 1 \text {; }
$$

note, however, that Eq. $(\mathrm{IV}-3)$ is invalid for hydrogen $(\mathrm{j}=1)$ :

$$
Z / A_{H}=0.992 \text {. }
$$

Let

$$
G_{z}=\beta_{z}-\alpha_{z}\left(1+\beta_{z}\right)
$$

such that

$$
\mathrm{n}_{\mathbf{z}} \sigma_{\mathrm{z}}=\left(N \rho / \mathrm{A}_{\mathrm{z}}\right) Z \sigma_{\mathrm{H}}\left(1+\beta_{\mathrm{i}}\right)=\mathbf{g} \rho\left(1+\mathrm{G}_{\mathbf{z}}\right), \quad \mathbf{g}=N \sigma_{\mathrm{H}}(661 \mathrm{keV}) / 2 .
$$

Rearranging Eq. (IV-1) and introducing these definitions, one finds, in the absence of hydrogen,

$$
\log \left(\frac{C_{0}}{C}\right)=\operatorname{gxp}\left[1+\sum_{z \min }^{\operatorname{zmax}} G_{z} \omega_{z}\right]
$$

when hydrogen is present, this becomes

$$
\begin{aligned}
& \log \left(\frac{C_{0}}{C}\right)=\operatorname{gxp}\left[1+m \omega_{H}+\sum_{z=2}^{z \max } G_{z} \omega_{z}\right], \\
& m=2 / A_{H}-1=0.984 ; \quad g=N \sigma_{H} / 2=.07711 .
\end{aligned}
$$

Photon removal cross sections at $E=0.661 \mathrm{MeV}$, correction factors $\beta$ for those cross sections, atomic masses A, correction factors $\alpha$ for those masses and overall correction factors $G$ for 32 elements are listed in Table IV-1. Cubic interpolation (on a logarithmic energy scale) between cross sections listed in ref. (G-4) was used to calculate $661 \mathrm{keV}$ values. FIG(IV-1) shows a plot of $\alpha$ and $\beta$ from $Z=3$ to 50. As Table and plot indicate, $\beta$ factors come to less than $1 \%$ for $Z \leq 16(\mathrm{~S})$; for $Z \geq 30(\mathrm{Zn})$, other photon removal processes, varying with higher powers of $Z$, come into play, hence, Eq. (IV-5) is no longer a good approximation. The $\alpha$ correction is negligibly small only for $\mathrm{C}, 0, N$, $\mathrm{Si}, \mathrm{S}$ and $\mathrm{Ca}$ and becomes large for $\mathrm{Z} \geq 21$, nuclear stability requiring an excess of neutrons (considerations of nuclear stability also explain the noticeable lowering of $\alpha$ for even-numbered elements). To sum up, corrections tend to be especially small for organics and for silicates, carbonates and sulfates, hence, many mineral industrial raw materials, whereas $\alpha$ is large for beryllium and lithium, ingredients of fusion blanket slurries, one of the scenarios where a need for an accurate on-line densitometer may develop. 
TABLE (IV-1): $661 \mathrm{keV}$ PHOTON REMOVAL CROSS SECTIONS AND DERIVED CONSTANTS FOR SELECTED STABLE ISOTOPES

\begin{tabular}{|c|c|c|c|c|c|c|}
\hline Elmt. & $\mathbf{z}$ & $A$ & $\sigma(.661)$ & $a$ & $\beta$ & G \\
\hline$H$ & 1 & 1.00789 & .256118684 & - & - & - \\
\hline He & 2 & - & - & - & - & - \\
\hline Li & 3 & 6.94100 & .769272386 & .135571243 & .001192590 & -.134540334 \\
\hline $\mathrm{Be}$ & 4 & 9.01218 & 1.026056424 & .112312448 & .001543902 & -.110941945 \\
\hline B & 5 & 10.81000 & 1.283907882 & .074930620 & .002588224 & -.072536333 \\
\hline C & 6 & 12.01100 & 1.535976726 & .000915827 & -.000478539 & -.001393928 \\
\hline $\mathbf{N}$ & 7 & 14.00670 & 1.793239275 & .000478343 & .000227845 & -.000250606 \\
\hline 0 & 8 & 15.99940 & 2.052514369 & -.000037501 & .001739866 & .001777433 \\
\hline $\mathbf{F}$ & 9 & 18.99840 & $2.30977691 \mathrm{E}$ & .052551794 & .002042786 & -.050616360 \\
\hline $\mathrm{Ne}$ & 10 & - & - & - & - & - \\
\hline $\mathrm{Na}$ & 11 & 22.98977 & 2.828317259 & .043052627 & .003908606 & -.039312297 \\
\hline $\mathrm{Mg}$ & 12 & 24.30500 & 3.088373565 & .012548858 & .004864073 & -.007745824 \\
\hline Al & 13 & 26.98154 & 3.345641038 & .036378205 & .004834942 & -.031719149 \\
\hline Si & 14 & 28.08550 & 3.610109837 & .003044276 & .006818341 & .003753309 \\
\hline$P$ & 15 & 30.97376 & 3.864186301 & .031438224 & .005832203 & -.025789375 \\
\hline $\mathbf{s}$ & 16 & 32.06000 & 4.129441219 & .001871491 & .007697183 & .005811286 \\
\hline $\mathrm{Cl}$ & 17 & 35.45300 & 4.398711399 & .040983838 & .010264950 & -.031139585 \\
\hline Ar & 18 & - & - & - & - & - \\
\hline $\mathrm{K}$ & 19 & 39.09830 & 4.922455274 & .028090735 & .011548980 & -.016866175 \\
\hline $\mathrm{Ca}$ & 20 & 40.08000 & 5.1825214 .33 & .001996008 & .011742165 & .009722719 \\
\hline Sc & 21 & & & & & \\
\hline $\mathrm{Ti}$ & 22 & 47.90000 & 5.718277860 & .081419624 & .014848729 & -.067779873 \\
\hline $\mathrm{Va}$ & 23 & & & & & \\
\hline $\mathrm{Cr}$ & 24 & 51.99600 & 6.259232911 & .076852066 & .018283271 & -.059973901 \\
\hline $\operatorname{Mn}$ & 25 & 54.93800 & 6.544928119 & .089883141 & .022171131 & -.069704821 \\
\hline $\mathrm{Fe}$ & 26 & 55.84700 & 6.812200506 & .068884631 & .022993355 & -.047475165 \\
\hline Co & 27 & & & & & \\
\hline $\mathrm{Ni}$ & 28 & 58.70000 & 7.379585502 & .045996593 & .029040994 & -.018291386 \\
\hline $\mathrm{Cu}$ & 29 & 63.54600 & 7.670479326 & .087275360 & .032721561 & -.057409584 \\
\hline $\mathrm{Zn}$ & 30 & 65.38000 & 7.958978142 & .082288162 & .035845052 & -.049392733 \\
\hline As & 33 & 74.92160 & 8.853672153 & .119079144 & .047534258 & -.077205225 \\
\hline $\mathrm{Sr}$ & 38 & 87.62000 & 10.456290590 & .132618124 & .074367312 & -.068113265 \\
\hline $\mathrm{zr}$ & 40 & 91.22000 & 11.113781091 & .122999342 & .084827249 & -.048605789 \\
\hline Mo & 42 & 95.94000 & 11.885444853 & .124452783 & .104904874 & -.032603612 \\
\hline $\mathrm{Ag}$ & 47 & 107.86800 & 13.712611943 & .128564542 & .139150399 & -.007303950 \\
\hline $\mathrm{Sn}$ & 50 & 118.69000 & 14.985286664 & .157469037 & .170183013 & -.014084579 \\
\hline $\mathrm{Hg}$ & 80 & 200.59000 & 36.264306793 & .202353058 & .769897565 & .411753379 \\
\hline $\mathrm{Pb}$ & 82 & 207.20000 & 38.505914857 & .208494208 & .833463769 & .451197192 \\
\hline
\end{tabular}




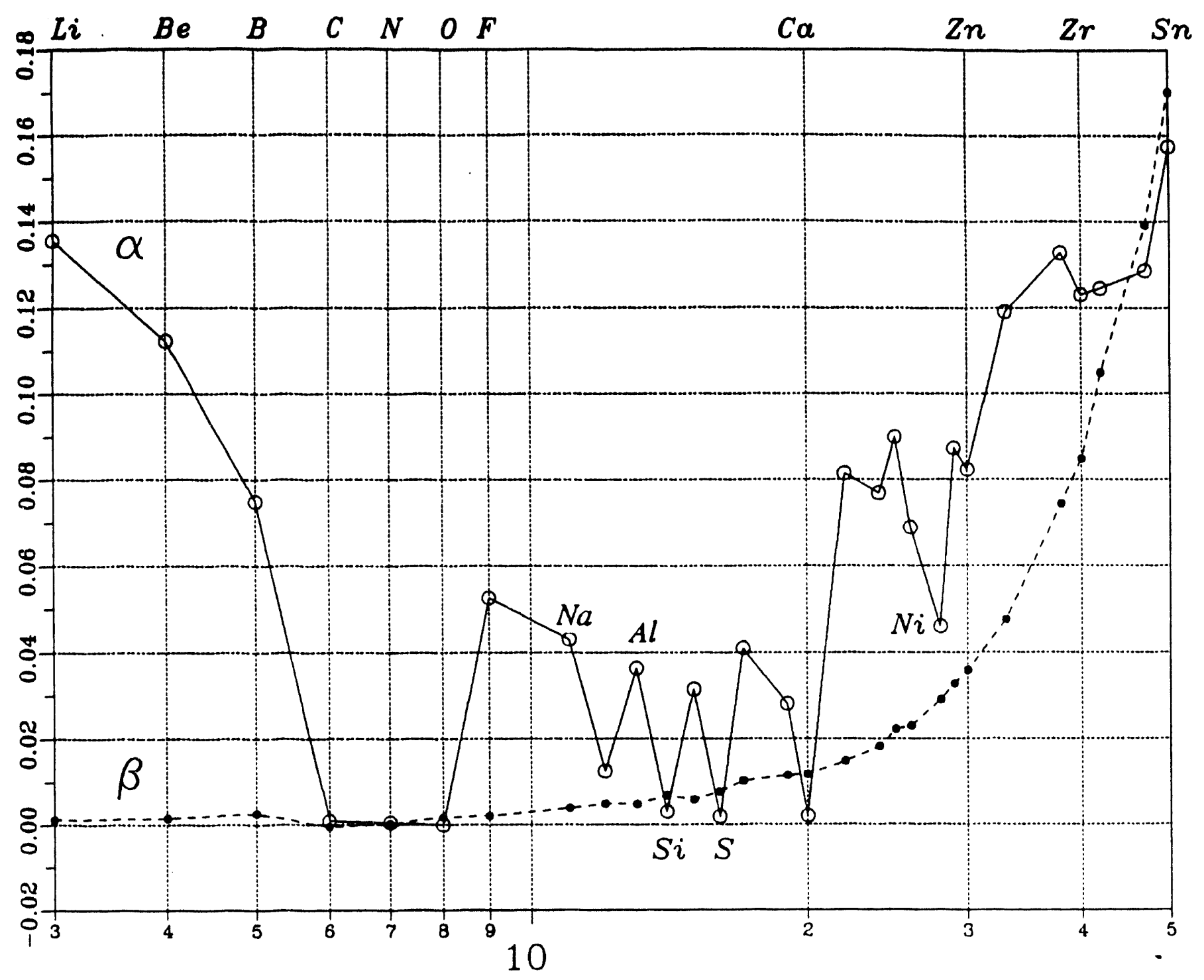

FIG(IV-1): Correction Parameters $\alpha$ and $\beta$ vs Atomic Number $Z$ (661 keV)

(3) Transmission through Quasi-homogeneous, Binary Mixtures

Now, let the medium consist of solid particles suspended in a fluid within a container of wall thickness $\Delta x_{w}$. Let

$\rho_{c}=$ container material density,

$\rho_{s}, \rho_{f}=$ intrinsic densities of solid and fluid, respectively, $\Delta \rho=\rho_{s}-\rho_{f}$,

$\rho_{m}=$ bulk density of the 2-phase medium,

$\phi_{s}=$ solid phase volume fraction, $\phi_{f}=$ fluid phase fraction,

and assume no gas phase is present: $\phi_{s}+\phi_{f}=1$; then we have, 


$$
\rho_{\mathrm{m}}=\rho_{\mathrm{r}}+\phi_{\mathrm{s}} \Delta \rho
$$

Let

$$
\begin{aligned}
\omega_{1 \mathrm{~s}}, \omega_{\text {Jf }} & =\text { elemental welght fractions in each phase, respectively, } \\
\omega_{k c} & =\text { elemental welght fractions of the container wall, }
\end{aligned}
$$

then the foregoing equations can be combined to yleld the relation

$$
\log \left(\frac{C_{0}}{C}\right)=\Delta x_{W} \sum n_{j w} \sigma_{j}+g \times\left[\rho_{g} \phi_{g} M_{s}+\rho_{f}\left(1-\phi_{g}\right) M_{f}\right] .
$$

where

$$
M_{s}=\left[1+m \omega_{H s}+\sum G_{1 s} \omega_{1 s}\right] ; \quad M_{f}=\left[1+m \omega_{H f}+\sum G_{f f} \omega_{f f}\right] .
$$

A reference measurement with the same container, fllled to height $x$ with pure liquid, yields

$$
\left.\log \left(\frac{C_{o}}{C_{f}}\right)=\Delta x_{w} \sum n_{j w^{\prime}}+g x \rho_{f} M_{f} \quad \text { (IV -9, }\right)
$$

whence, subtracting the reference log ratio from the medium present log ratio, one obtains a direct relation of the phase fraction $\phi_{s}$ to $C$ and $C_{f}$ :

$$
\begin{array}{ll}
\phi_{s}=\frac{1}{A g X} \log \left(\frac{C}{C_{f}}\right) ; & (I V-10) \\
a=\Delta \rho[1+a+b], \quad \Delta \rho=\rho_{s}-\rho_{f} ; & (I V-11 \mathrm{a}) \\
b=m\left[\rho_{s} \omega_{H s}+\rho_{f} \omega_{H f}\right] / \Delta \rho, & (I V-11 b) \\
b=\left[\rho_{s} \sum_{1 s} \omega_{1 s}-\rho_{f} \sum_{k f} \omega_{k f}\right] / \Delta \rho . & (I V-11 c)
\end{array}
$$

$\mathbf{a}$ and $\mathbf{b}$ are compound parameters that can be calculated from constants $m, g, x$, laboratory measurements (phase densities), Ultimate analysis (hydrogen fractions), cross section data and additional Major Constituent Elemental Analyses or a Proximate Analysis, as discussed in the next Chapter.

A numerical example: let $x=5 \mathrm{~cm}, \rho_{\mathrm{s}}=1.4, \rho_{\mathrm{f}}=1.0$ (coal-water), $\omega_{\mathrm{Hs}}=$ $0.05, \omega_{H f}=0.111$ hence $\mathbf{a}=-0.101 ; 1+\mathbf{a}=0.899 ; \mathbf{g x}=.3855 ;$ neglect $\mathbf{b}$ then

$$
\mathbf{g A x}=0.139 \text { at } 661 \mathrm{keV} \text { photon energy. }
$$


A note on the application of Eq. (IV-10): in slurry conveyance, phases can and do interact chemically in transit, e.g. water-leached minerals become part of the fluid phase but are accounted for in the phase fraction calculation as part of the solid. If the slurry serves to convey a solld reagent into a Chemical reactor, this actually yields a correct final result: upon evaporation, leached components once more join the solid fraction.

\section{(4) Extension to Three-phase Quasi-homogeneous Media}

In typlcal 3-phase, quasi-homogeneous slurries (fluid/solid/entrained gas, well-mixed), the gas fraction can be large enough to make Eq. (IV-8) inadmissible. For that case,

Put

$$
\rho_{m}=\rho_{s} \phi_{s}+\rho_{f} \phi_{f^{\prime}} \quad \phi_{s}+\phi_{f}=1-\phi_{g} . \quad \text { (IV-8') }
$$

$$
B_{x}=1+m \omega_{H x}+\sum_{1 x} \omega_{1 x} ; \quad x=s, f ;
$$

then, from the foregoing,

$$
\frac{1}{g x} \log \left(\frac{C_{f}}{C}\right)=\rho_{f} \phi_{f} B_{f}+\rho_{s} \phi_{s} B_{s} .
$$

Suppose, now, that another source, of different energy, for which a different set $\alpha, \beta, f$ obtains, has been installed in another well-collimated source-detector unit. For that unit, designated by stars, the constant

$$
\mathrm{B}_{\mathrm{x}}^{*}=1+m \omega_{H x}+\sum_{1 x}^{*} \omega_{1 x} ; \quad x=s, f,
$$

is associated with count $C^{*}$ and reference count $C_{f}^{*}$ :

$$
\frac{1}{g x} \log \left(\frac{C_{f}^{*}}{C^{*}}\right)=\rho_{f} \phi_{f} B_{f}^{*}+\rho_{s} \phi_{s} B_{s}^{*} ;
$$

rearranging and combining counts,

$$
\phi_{s}=\frac{1}{g \times\left[B_{s} / B_{f}-B_{s}^{*} / B_{f}^{*}\right]}\left[\frac{1}{B_{f}} \log \left(\frac{C_{f}}{C}\right)-\frac{1}{B_{f}^{*}} \log \left(\frac{C_{f}^{*}}{C}\right)\right] \quad\left(I V-10^{\prime}\right)
$$

for a 3-phase medium without significant phase separation (the effect of phase separation is considered further on). A similar type of procedure and data processing routine has been used for a laboratory determination of the carbon, ash and moisture content of coal samples (G-5). 


\section{(5) Data Processing Options}

As already mentioned, conventional on-line gamma gauging, using standard commercial instrumentation, relles on calibration: the installation geometry is duplicated, a representative number of carefully made-up solid - liquid mixtures is poured into the mockup; the instrument readout current is recorded for each mixture and plotted against the Solid fraction, thus obtaining a "calibration curve". The plot, covering a limited range of bulk densities, is usually fitted with a straight line: expanding the logarithm in Eq. (IV-10),

$$
\begin{aligned}
\log \left(C / C_{f}\right) & =\log \left[1+\left(C-C_{f}\right) / C_{f}\right]=\frac{C-C_{f}}{C_{f}}\left(1-\frac{1}{2} \frac{C-C_{f}}{C_{f}}+\frac{1}{3}\left(\frac{C-C_{f}}{C_{f}}\right)^{2} \ldots\right) \\
& =Q \phi_{s} ; \quad Q=g A x,
\end{aligned}
$$

hence,

$$
\mathrm{C}=\mathrm{C}_{\mathrm{f}}+\mathrm{Q} \mathrm{C}_{\mathrm{f}} \phi_{\mathrm{s}}+\left[\mathrm{Q}^{2} \mathrm{C}_{\mathrm{f}} / 2\right] \phi_{\mathrm{s}}^{2} \ldots=\mathrm{A}+\mathrm{B} \phi_{\mathrm{s}}+\mathrm{C} \phi_{\mathrm{s}}^{2} \ldots
$$

Dropping the second-order term yields a quasi-linear relation between measured transmission current $C$ and solid phase fraction, requiring constants $A$ and $B$ to be determined by callbration as outlined above(*). Unfortunately, calibra$t i o n$ applies only to one specific mixture and must be repeated for a somewhat different mixture - say an aqueous suspension of coal from a different mine. As both coal Combustion and Chemical processing (gasification, liquefaction) plants routinely use coal from many different mines, accurate composition measurements that make use of conventional equipment would require quasicontinuous calibration; since that is evidently impractical, measurements are, too often, inaccurate. This can be remedied by a Good-Geometry, energydiscriminating on-line Gamma Transmission device.

The accuracy of the medium composition calculation (Eq. (IV-10)) critically depends on accurate intrinsic density data. For heated coal slurries, the "intrinsic density" of coal is best measured by carefully weighing out several samples, mixing these with weighed amounts of fluid, then measuring the volume (e.g., with a sonic level gauge) after bringing the slurry sample to conveyor temperature. This can be compared with results obtained with a gas pycnometer. The Hydrogen weight fraction, also required, can be roughly estimated from the Rank of the coal, noting that the atomic hydrogen/ carbon ratio is 0.5 for anthracite $(90 \% \mathrm{C})$ and 1.05 for Lignite $(30 \% \mathrm{C})$. However, automatic and semiautomatic Proximate and Ultimate Analysis Instruments, introduced in the last few years, together with sampling cutters and classifiers, now offer an accurate as well as convenient determinaton of the hydrogen weight fraction.

Proximate analysis, whlle not useful for the Hydrogen Fraction, yields a reasonably good estimate of Factor $b$ from the Ash Fraction it reports, if a "standard Ash Composition" is assumed. Some numerical examples are provide in Chapter IV.

(*) Since a PC-type computer can nowadays be provided at modest cost, this linearity assumption, convenient when computation was more difficult and expensive, is no longer necessary or desirable. 


\section{(6) Effect of Non-uniform Cross-Beam Phase Distributions}

Non-uniform phase distributions, ranging from a slight phase gradient to full separation into two layers, have a strong effect on the readout of EM sensing (and other) densitometers, considered further on. For gamma transmission,

mon-uniform phase distributions along the beam have no effect whatever,

mphase non-uniformity across the beam requires correction.

In the presence of a cross-beam phase distribution, represented in the following by the duct-average value $\langle\phi\rangle$ and local deviation from average, $\Delta \phi(y)=\phi(y)-\langle\phi\rangle$, the local (narrow-beam) measurement $C(y)$ can be expressed as the product of a stationary component $C_{s t}$ and a fluctuating factor,

$$
\begin{gathered}
C(y)=C_{f} e^{-Q \phi(y)}=C_{f} e^{-Q[\langle\phi\rangle+\Delta \phi(y)]}=C_{s t} e^{\Delta \phi(y),} \\
C_{s t}=C_{f} e^{-Q\langle\phi\rangle},
\end{gathered}
$$

hence, averaging by integration,

$$
\langle C\rangle=\frac{1}{Y} \int C(y) d y=C_{s t} \int \exp [-Q \Delta \phi(y)] d y / Y,
$$

where $y$ is the cross-beam direction, $Y$ the beam width; carets $<>$ denote averaging. Expanding the exponent and integrating term by term,

$$
\langle C\rangle=C_{s t}\left\{1+Q^{2}\left\langle\Delta \phi^{2}>/ 2 \ldots\right\}\right.
$$

or, to second order,

$$
\log \left[\frac{C_{f}}{\langle C\rangle}\right]=Q\langle\phi\rangle\left[1-Q\left\langle\Delta \phi^{2}\right\rangle / 2\langle\phi\rangle\right]
$$

where $\left\langle\Delta \phi^{2}\right\rangle=$ second moment of the cross-beam solids distribution. To estimate the magnitude of the correction term, we may use a value for $\mathbf{Q}$ of.1542 (coalwater, cf. above) and consider a strong phase gradient $\Delta \phi / \phi=0.8$ at $\langle\phi\rangle=0.4$ (slurry pipeline), yielding an error of $2 \%$ according to Eq. (IV-14). For $\Delta \phi / \phi=$ 0.4 , the error comes to $0.4 \%$, for smaller gradients, the error is negligible. Since slurry pipelines must be designed to avoid the formation of a bottom layer, the worst-case estimate of $\Delta \phi / \phi=0.8$ is perhaps unrealistic; in any case, cross-beam error can be eliminated by vertical beam siting. The unavoidable error resulting from the central Magnus-effect concentration of solids can be estimated at $<0.5 \%$. Typical phase non-uniformity in dense-slurry conveyors, featuring over $50 \%$ solids, is too small to make the correction significant. 
Another error, caused by fluctuations in $t i m e$, affects all instruments whose readout is not linearly related to the phase fraction, hence, both gamma transmission and EM interrogation. This error is therefore discussed in section (C) of this Chapter, after reviewing EM sensing.

\section{(7) Statistical error}

The statistical error arising from the Poisson fluctuation of counts is readily estimated by elementary methods. As further discussed below, all photon selection processes (collimation, scattering/absorption in duct walls, medium or pure fluid, conversion in the scintillator and photomultiplier to voltage pulses and selection/rejection of these by the pulse-height discriminator) amount to Bernoulli "games" with a yes/no result. Except for interactions with the flowing medium (which requires special treatment, cf. section (C) below), processes can be treated as a single game and concatenated with the Poisson process that selects the number of photons emitted in the counting interval. This results in overall Poisson statistics, with mean and variance equal to the product of averages. Let

$\mathrm{n}=$ photon rate detected with empty container,

$\mathrm{c}_{\mathrm{f}}=\mathrm{nP}_{\mathrm{f}}=$ photon rate detected with container + fluid,

$\mathrm{c}_{\mathrm{m}}=\mathrm{nP}_{\mathrm{m}}=$ photon rate detected with container + medium,

$P_{m}, P_{f}=$ photon interaction probabilities in medium and pure fluid,

then for a medium count interval $t$ and reference (pure fluid) count interval $t_{f} \gg t, N=n t, C_{f}=c_{f} t_{f}$ and $C_{m}=c_{m} t$ are all Poisson-distributed with variances equal to mean values,

$$
\operatorname{var}(\mathrm{N})=\langle\mathrm{N}) ; \quad \operatorname{var}\left(\mathrm{C}_{\mathrm{f}}\right)=\mathrm{C}_{\mathrm{f}} ; \quad \operatorname{var}\left(\mathrm{C}_{\mathrm{m}}\right)=\left\langle\mathrm{C}_{\mathrm{m}}\right\rangle .
$$

To express the readout $R$ in comparable counts, as in the foregoing, $\left\langle\mathrm{C}_{f}\right\rangle$ is scaled to count interval $t$ : let $\left\langle C_{f}^{\prime}\right\rangle=\left\langle C_{f}\right\rangle\left(t / t_{f}\right)$, then

$$
R=\frac{1}{Q} \log \frac{\left\langle C_{f}^{\prime}\right\rangle}{\langle C\rangle}=\langle\phi\rangle \text {. }
$$

The variance $\operatorname{var}(R)$ in the readout is readily calculated by propagation, where now $\operatorname{var}\left(C_{f}^{\prime}\right)=\left(t / t_{f}\right)^{2} \operatorname{var}\left(C_{f}\right)$ :

$$
\operatorname{var}(R)=\left(\frac{\partial R}{\partial C_{m}}\right)^{2} \operatorname{var}\left(C_{m}\right)+\left(\frac{\partial R}{\partial C_{f}^{\prime}}\right)^{2} \operatorname{var}\left(C_{f}^{\prime}\right)=\frac{1}{Q^{2} C_{m} t}\left[1+\frac{C_{m} t}{C_{f} t_{f}}\right]
$$

hence, the fractional statistical error $E=\sqrt{\operatorname{var}(R)} /<R>$ comes to 


$$
E(R)=\left(\frac{1}{\sqrt{C_{m} t Q}\langle\phi\rangle}\right) \sqrt{1+\frac{C_{m}}{C_{f}}} .
$$

Once more estimating $\mathbf{Q}=.1542$ for a coal/water slurry, let $\langle\phi\rangle=0.6$. The ratio of counts in the square root can be made negligibly small by a relatively long reference count, say $t_{f}=400 \mathrm{t}$. A count rate $c_{m}=2.5 \times 10^{5}$ is feasible with wideband electronics. A 1 -second readout (count) interval t thus implies a $2 \%$ statistical readout error, reduced to $1 \%$ by a 4 -second interval. The error worsens for smaller $\langle\phi\rangle$ and density contrast $\Delta \rho$ and improves for a longer path $x$, hence, for larger ducts.

\section{(8) Summary.}

While available, conventional gamma transmission equipment provides online densitometry that is adequate in many industrial scenarios, calibration difficulties that arise in dense slurry conveyors suggest a different approach, utilizing equipment and data processing options that, although by no means unrecognized, have not been fully exploited in laboratory equipment and scarcely acknowledged at all in the design of industrial instrumentation:

(1) With careful collimation and an energy-selective gamma detector, photon interaction theory accurately predicts beam attenuation by the medium, relative to a reference count with medium absent, in terms of constants and medium composition information;

(2) The entrained phase fraction is directly proportional to the logarithm of the ratio of counts with pure entraining phase present to counts with medium present. For a two-phase medium consisting largely or entirely of light elements, minerals or organics, the constant of proportionality scales with the medium density contrast and path length through the medium; the hydrogen weight fractions of both phases furnishes a small correction; an even smiller correction can be based on a chemical analysis of both phases or a proximate analysis of the entrained material and chemical formula of the entraining fluid. This permits one to dispense with the conventional instrument readout calibration for a specific installation and two-phased medium.

(3) Errors due to cross-beam phase non-uniformity are small and/or can be minimized by vertical beam orientation on a long horizontal duct, taking advantage of the fact that in-line non-uniformity causes no readout error for gamma transmission densitometry. To keep statistical error within $1 \%$ calls for careful design o the electronics.

(4) To obtain the solids feedrate, i.e., the information needed for process control, additional on-line computations and a companion flow velocity or mass flowrate readout are required. A flow velocity measurement can be provided through cross-correlation, using a pair of non-intrusive sensors. Gamma transmission is marginally capable of providing this service for very coarse and dilute slurries, but fails in dense, fine-consist slurries. 


\section{(C) F.M Field Sensing}

\section{(1) Introduction}

E.M.Field or "Capacitive" sensing amounts to a reading of the bulk dielectric constant, averaged over the entire region traversed by field lines that terminate on the "signal electrode" of an electrode pair. For a rectangular container, used by feedhopper moisture meters and similar devices (e.g. in the cereal processing industry), flat electrodes that form two opposite sides of the container, defining a Parallel Plate (Cartesian) geometry, provide a field that is perfectly uniform within and does not stray outside the container, thus sensing the medium without bias. Such high field uniformity and sharp delineation of the sensed region is not achievable for cylindrical containers (ducts of circular cross section). A variety of electrode configurations. designed to pass most of the field lines that terminate on the sional electroce. through the duct interior in fairly uniform fashion, has been described in the instrumentation literature. If the medium within the duct is strictly homegenecus, many of these configurations are adequate, whether the objert of measurement is moisture or composition (bulk density) analysis. Similarly, a pair of such capacitors deployed in a cross Correlation scheme an produce a ransonably accurate reading of a near-uniform (very high Reynolds' number) flow velocity.

However, the phases of real binary media such as flowing slurries tend be non-uniformly distributed; moreover, the radial velocity distribution patterns that characterize duct flow vary considerably with the Reynolds' number. Unier these realistic circumstances, error-minimizing FM Field sensing roquires that

* the entire duct cross section is covered by and lies within the field,

* field lines ending on the signal electrode do not pass outside the duct,

* the field within the duct is highly uniform, noting that the local sensing efficiency varies with the square of the local field strength.

To investigate how well existing capacitive devices satisfy these requirements, equipotentials were plotted for six representative capacitor geometries by interpolation on potential maps produced by solving Poisson's equation. This study, presented below, leads to the conclusion that none really come close to meeting all three specifications. The choice of $\mathrm{EM}$ sensing geometry offered is thus, quite simply, between a rectangular measurement insert into a circular duct, that can place a uniform field over the entire cross section, and a device conforming to a circular profile, for which error is inevitable: at best, the electrode geometry can be chosen so as to produce the least error. For dense, fine-consist slurries, featuring close-to-uniform phase mixing and laminar, non-Newtonian flow, sensing uniformity is relatively unimportant. in density measurement but especially important in velocity measurement. (e.g. . the Cross Correlation-based scheme discussed below). For more dilute, coarse consist slurries, flowing in quasi-turbulent mode, the opposite applies: solids settling and non-uniform sensing can produce large bulk density readout errors, while a close-to-uniform medium velocity allows a relatively accurato velocity measurement (as discussed further on). 
Bulk density (phase fraction) determination by means of EM Field sensing requires a reference measurement (duct filled with pure fluid): together, the two measurements allow the conversion of the readout signal, proportional to the device capacity (as discussed in the following section on Input Circuit configuration), to the bulk dielectric constant. The desired Phase Fraction can be calculated from the latter by making use of the theory of binary dielectric mixtures, also presented below(*). A final topic, the effect of phase non-uniformity, is taken up in the concluding section. Other matters, such as electronics stabilization, data processing and hardware design, are covered in the following chapters, on On-line densitometry and on Cross-Correlation Velocity measurement.

\section{(2) Input Sircuit: Dielectric Constant and Readout Signal}

Channel electronics design may seem like a trivial matter; yet, an improper choice of circuitry and driving frequency can make the readout signal sensitive to parasitic capacities and/or medium conductivity, warranting the following brief discussion. Input circuit details shown in FIG (IV-2) include a Guard strip flanking the Signal Electrode, stray parallel and series elements, as well as Feedback in shunt with the Operational Amplifier. Amplifier gain, Feedback components and frequency $\omega$ comprise disposable elements whose choice determines tc what degree the readout is proportional to the signal electrode capacity, hence, to the bulk dielectric constant of the medium within that capacitor, with a known constant of proportionality. In addition to a measurement with medium present, a reference measurement (capacitor filled with pure fluid) is assumed to have been made and recorded, or else, a dummy capacitor filled with pure fluid has been provided, as discussed further on. Let

$$
\begin{aligned}
& C=\text { capacity of empty capacitor, } \\
& C_{f}=\text { capacity as read with region filled with carrier fluid, } \\
& C_{f s}=\text { capacity as read with uniform fluid/solid mixture, } \\
& C_{S}, R_{S}=\text { capacity \& resistance appearing in series with } C_{f s} \text {, } \\
& C_{F}, R_{F}=\text { feedback capacity and resistance, } \\
& R=\text { resistance appearing in shunt with } C, C_{f} \text { or } C_{f s}, \\
& T_{F}=R_{F} C_{F} ; T_{S}=R_{S} C_{S} ; T_{p}=R_{S}\left(C+C_{S}\right) /\left(R+R_{S}\right) ; T=R C ; \\
& E=\text { amplitude of driving } A C \text {, } \\
& e_{0}=\text { Signal, solid/fluid mixture, } \\
& e_{o f}=\text { Signal reference measurement, fluid only, } \\
& R_{s} \text {, } K_{f}=\text { intrinsic dielectric constants, solid and fluid, } \\
& R_{m}=\text { medium bulk dielectric constant; }
\end{aligned}
$$

(*) This important topic needs special emphasis as it has been largely ignored in E.M. sensing densitometer design. Instead, an ad-hoc assumption of linearity, at variance with all that is known about bulk properties of binary mixtures, is too of ten the basis of equations presented in reports or brochures. 


$$
\begin{aligned}
& \omega=2 \pi f ; f=\text { driving frequency, } \\
& h_{j}=1 / \omega T_{j}, \quad j=S, F, p ;
\end{aligned}
$$

then, in the absence of a dielectric medium between the signal plates, Kirchhoff's laws yield the real component

$$
-e_{0}=E \frac{R_{F}(1+\omega T)\left(1+\omega T_{S}\right)}{\left(R+R_{S}\right)\left(1+\omega T_{F}\right)\left(1+\omega T_{p}\right)} .
$$

The amplitude thus comes to

$$
-e_{0}=E \frac{C}{C_{F}}\left[\frac{C_{S}}{C+C_{S}}\right]\left[\frac{\left(1+h^{2}\right)\left(1+h_{S}^{2}\right)}{\left(1+h_{F}^{2}\right)\left(1+h_{p}^{2}\right)}\right]^{1 / 2} .
$$

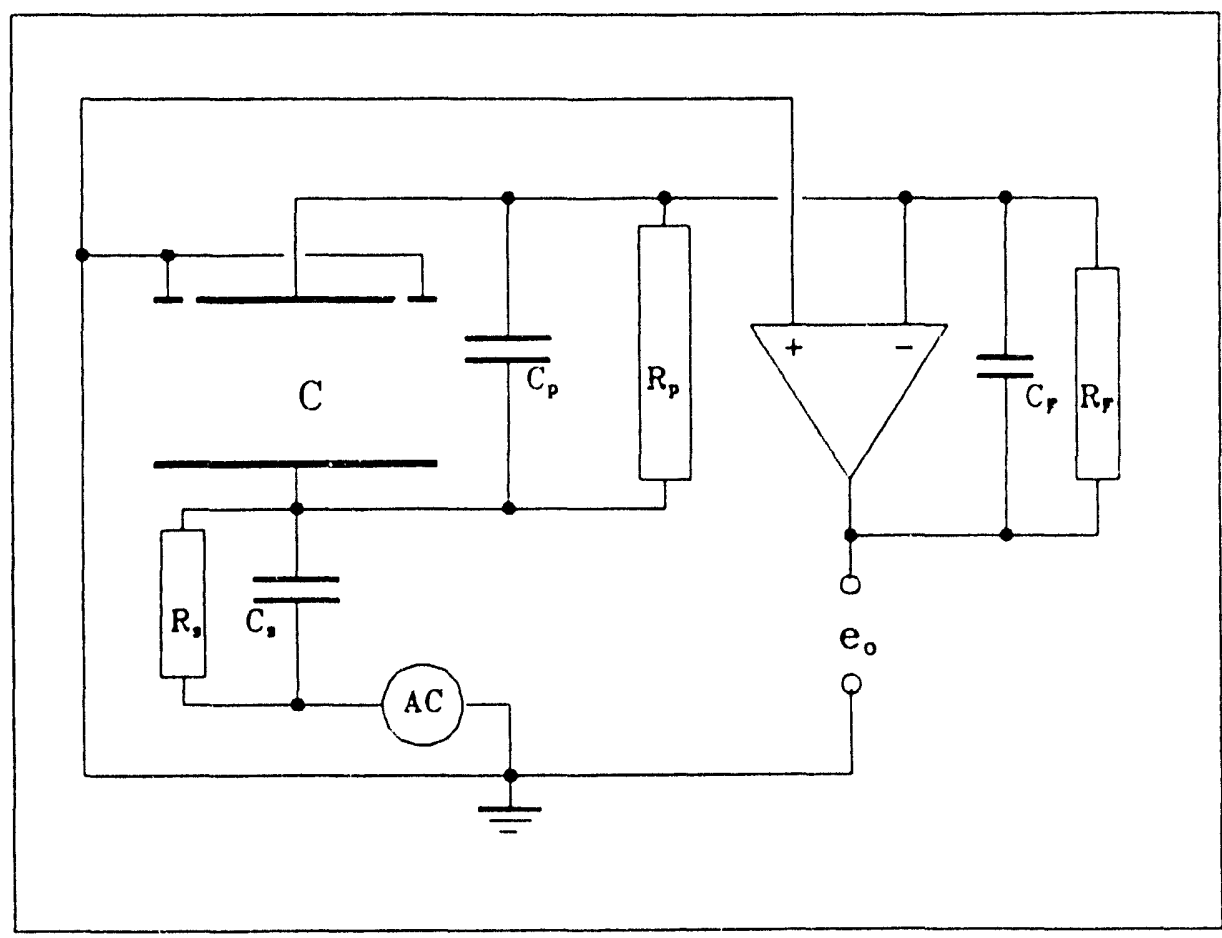

FIG (IV-2): E M Sensing Input Circuit, schematic

To make the series capacity correction factor within the first bracket,

$$
\frac{C_{S}}{C+C_{S}}=\frac{1}{1+C / C_{S}} \text {. }
$$


equal to unity, it is expedient to design the sensing device with electrode plates in direct contact with the medium. To make the second bracket close to unity, factors $h=1 / 2 \pi f R C$ must be made small: this is ensured by the choice of a high frequency $f(*)$ and large shunt resistance $R_{F}$.

Consider now a measurement with a binary mixture and a reference measurement with pure fluid, yielding the read t.s

$$
\begin{aligned}
& e_{0}=E\left(C_{s f} / C_{F}\right) \ldots 22 \text { phases present, } \\
& e_{\text {of }}=E\left(C_{f} / C_{F}\right) \ldots \ldots \text { reference measurement, }
\end{aligned}
$$

then

and

$$
\frac{e_{o}-e_{o f}}{e_{o f}}=\frac{C_{s f}-C_{f}}{C_{f}}=\frac{k_{m}-k_{f}}{k_{f}}
$$

$$
k_{m}=k_{r} \frac{e_{o}}{e_{o f}},
$$

relate readout and bulk dielectric constant. Eq. (IV-20) implies that the Field be identical for readings $e_{o}$ and $e_{o r}$. As discussed in Chapter $v$ under the rubric "dielectric discontinuity error, this is only approximately true when electrodes conform to a circular duct cross section.

\section{(3) Solving the Poisson Equation}

For an electrode system emplaced on a duct, it is expedient to extend signal + lateral guard electrodes more than one duct diameter in the direction parallel to the duct axis. A design study may thus reasonably model the capacitor in two dimensions, simplifying the Poisson equation,

$$
\partial^{2} V / \partial x^{2}+\partial v^{2} / \partial y^{2}=0
$$

or, in cylindrical coordinates,

$$
(1 / r) \partial / \partial r(r \partial V / \partial r)+\left(1 / r^{2}\right)\left(\partial^{2} V / \partial \vartheta^{2}\right)=0
$$

and allowing use of elementary Finite Difference routines for its solution; alternatively, Overrelaxation can be used(E-1), (SQ-7)-(SQ-10). All schemes require the complete specification of boundary potentials and proceed to calculate the potential $V(m, n)$ at mesh points separated by either uniform or otherwise specified intervals within the boundary. The iterative calculaton

(*) In this connection, we note that, contrary to what may be believed, EM sensing can be effectively applied to weakly conducting as well as to dielectric media, provided only that suitably wideband analog electronics are provided for the input stage (50-100 MHz). The alternative, a conauctivity measurement, seems a less effective choice, requiring several sets of electrodes and involving large corrections for medium temperature. 
may start with a small number of points, solve the Poisson equation and then double the number, once more solve the Poisson equation etc., as described in full detail in ref.(SQ-9). A preferred alternative is a quasi-linear initial approximation of the potential over the full mesh, followed by repeated iteration. The number of iterations needed to reach stable values does not seem to vary greatly with the initial approximation, rather, the first few iterations always result in large corrections while subsequent corrections converge slowly. The mesh density must consider the next step, tracing equipotentials on the map by interpolation: here, linear interpolation is straightforward, quadratic-cubic interpolation becomes difficult when equipotentials turn. One would thus want to make mesh intervals commensurate with turning radius and/or interval between successive equipotentials. Field lines are similarly traced from a selected starting point on an equipotential boundary by fitting a circular arc so that it intersects both equipotentials normally.

This defines a set of adjacent, curving field strips of variable width $\Delta w$ connecting the electrodes. The series-capacity of each strip (for a homogeneous dielectric of constant $k$, the reciprocal of the sum $\Delta h / k \Delta w, \Delta h=d i s-$ tance between adjacent equipotentials) can be calculated. The signal capacity of the chosen geometry is the sum of strip capacities. Finally, the local sensing efficiency and overall average sensing efficiency are computed. When electrodes bound regions of different dielectric constant, further modifications of the outlined computation routine are required.

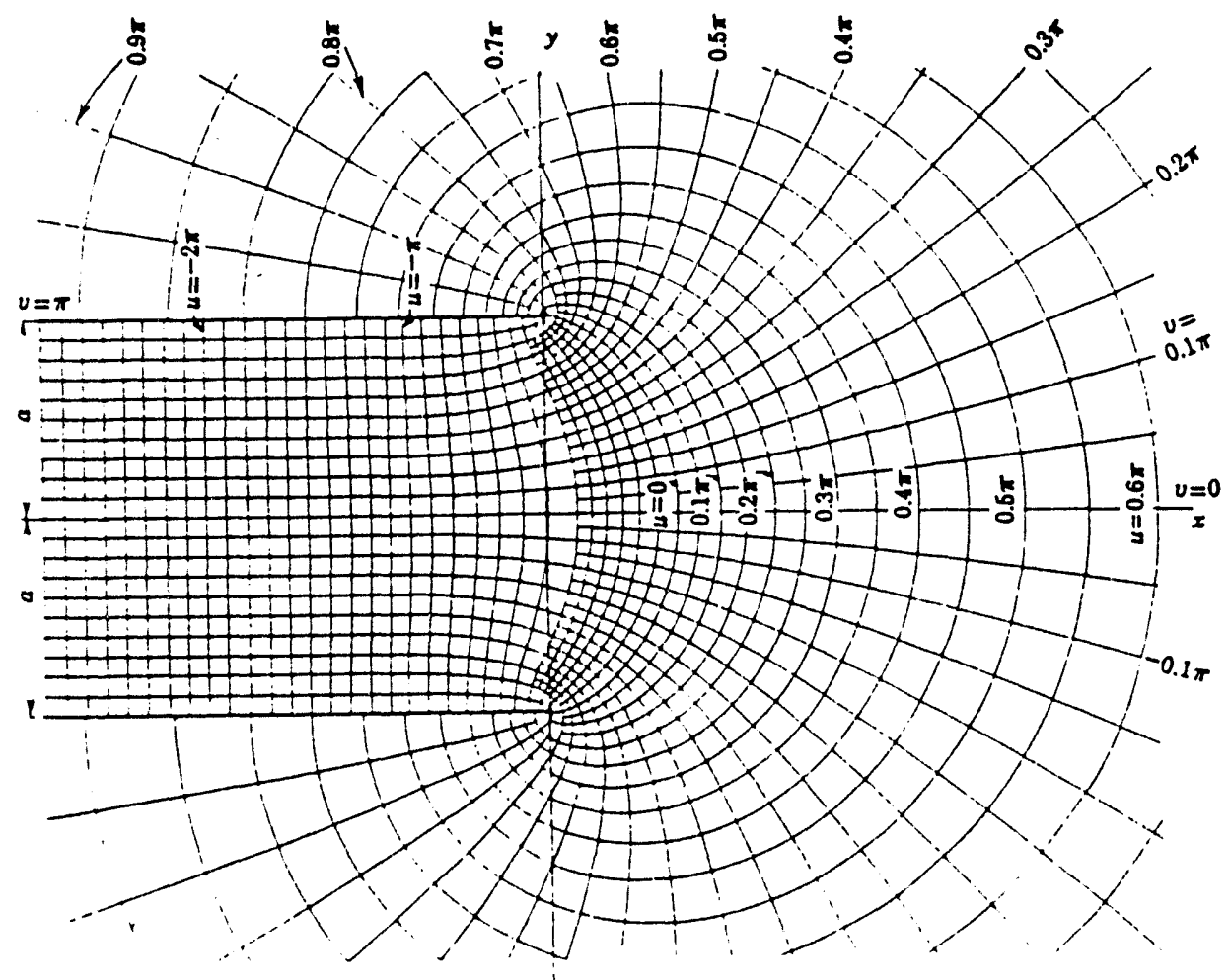

FIG (IV-3): Equipotentials and Field Lines near the edges of a Parallel-Plate Capacitor, via Schwartz Transformation. 
Some schemes (e.g., wrapped-plate) are more readily set up in Cartesian geometry; after finding equipotential and fleld line points, the metric is transformed to polar, requiring the Cartesian ordinate to be expressed as the logarithm of the radius (as discussed in the quoted references and incorporated into FORTRAN programs WP. FOR, excerpted in Appendix B). Other complex-plane transforms, of which several are given by Moon and Spencer(E-1), are of ten useful; as an example, FIG (III-3) (taken from ref $(E-1)$ ), shows the equipotentials and field lines at the edge of a parallel-plate capacitor.

\section{(4) Equipotentials for Representative Electrode Geometries}

Applying the equipotential calculations discussed in the preceding section, we now investigate a number of representative electrode configurations. The first of these, the ANL Mk 1 capacitor $(E-2)$, trying to impose the field uniformity of a Cartesian geometry on a circular duct, surrounds the duct with an arrangement of parallel plates. The second example is the widely used "wrapped-plate" scheme $(E-3),(E-4)$, the third, a "Patch" signal electrode configuration(E-5), (E-6). Strongly biased to sense the peripheral flow, the "adjacent Ring" geometry(E-7) and Spiral-Wrap geometry(E-8)-(E-11) are designed for azimuthal flow averaging in the case of phase separation. Instead of spiral strips, the Auburn International Inc. device(E-12) rotates the signal and driving electrodes among six strips, parallel to the duct axis, on the duct periphery. Ref. (E-13) is an example of ad-hoc equipotential tracing unsupported by Poisson equation solving. Lastly, ref.(E-14), an improved Wrapped-Plate scheme, bypasses the worst inhomogeneity through "guard" strips.

\section{(a) ANL Spoolpiece\#1 (External Parallel-Plate E Shims)}

This device, designed by $W$. W. Managan, is shown in FIGS (IV-4)-(IV-6). A long electrode and a pair of short electrodes of the same cross-sectional geometry was provided for phase fraction and for Cross-Correlation velocity sensing, respectively. First tested in a series of on-line runs at the HYGAS facility, IGT(E-15), a prototype coal gasifier that did not provide means of calibrating the readout, this device was thoroughly investigated in a series of coal-oil slurry runs at the ANL SLTF(E-16). Phase fraction measurements were compared to Grab-Samples, CCF/velocity measurements to Pulsed Neutron Activation as well as full-flow diversion measurements over a wide range of flowrates and phase fractions tc 0.6. Phase fraction results seemed acceptable, whereas velocity measurements (based on the conventional P.L.O. method discussed further on), were inconsistent throughout the series of runs and became especially erratic as laminar non-Newtonian flow set in at solid fractions above 0.5. Some problems with the device had already been found in the preliminary trials at the HYGAS facility, leading to the design studies described in ref. (E-2). The design objective to make the Fleld highly uniform throughout the duct volume was vindicated in these calculations, as demonstrated by FIGS. (IV-5) and (IV-6), calculated for medium dielectric constants $\mathrm{k}=4$ and $\mathrm{k}$ $=60$, respectively. However, these calculations also confirmed the suspicion that the sensed strip width, defined by field lines that just reach the signal electrode, varies with $k$, such that different parts of medium and adjacent electrodes contribute to "medium present" and "pure fluid" reference measurements. Moreover, field lines will shift so as to make stray capacity to the 
adjacent shim part of the readout signal.

Two separate issues emerge from these observations: first, stray capacity reading, secondly, the issue of sampling bias due to reading within a strip laid across a circular duct. To discuss the latter question in a preliminary way, consider a strip near the center of the electrodes where field lines are approximately parallel. Let $a=$ strip width and $E(r)=$ fraction of the annular region between $r$ and $r+\Delta r$ sensed by the strip, then

$$
\begin{array}{ll}
E(r)=1.0, & r \leq a, \\
E(r)=(2 / \pi) \arcsin (a / r), & R \geq r>a ;
\end{array}
$$

As $E(r)$ declines steeply with increasing $r$, the readout is biased towards the central region. Because strip reading is also featured by other capacitive sensing schemes (e.g., the rotating field scheme of Ref.(E-12)), a brief discussion of its performance in turbulent flow may be in order. The quasi-turbulent velocity distribution of slurry particles entrained along a duct of circular cross section can be fairly represented by the Prandtl equation,

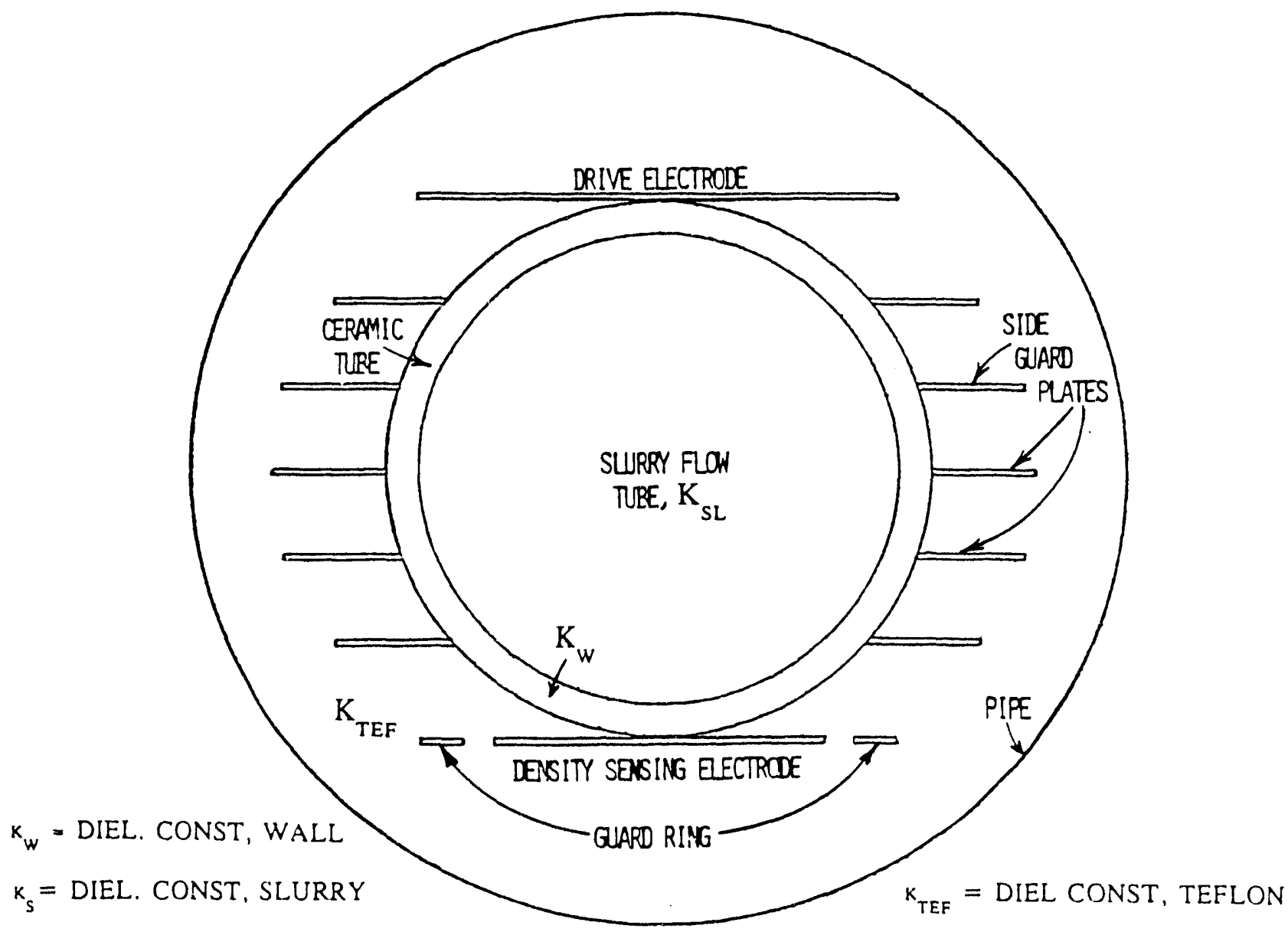

FIG (IV-4): ANL Mk-1 Geometry 


$$
U(r)=\left(\frac{y}{R}\right)^{1 / n}, W(V) d V=-2 n U^{n}\left[1-U^{n}\right] \frac{d V}{V}
$$

$V_{c}=$ centerflow velocity, $U=V(r) / V_{c}$;

$R=$ duct radius, $y=R-r$, the distance from the wall,

$\mathrm{n}=$ index varying from 5 to 10 for turbulent flow,

$W(V)=$ velocity distribution,

or corresponding transit time distribution over path $Z=V_{c} t_{c}=V(r) t(r)$,

$$
\tau(r)=\left(\begin{array}{l}
R \\
y
\end{array}\right]^{1 / n}, \quad W(\tau) d \tau=\left[2 n / \tau^{n}\right]\left[1-\frac{1}{\tau^{n}}\right] \frac{d \tau}{\tau}, \tau=t / t_{c} .
$$

The average velocity $\bar{V}$ and transit time $\bar{t}$ can be calculated from either distribution: for example,

$$
\bar{v}=\int_{t_{c}}^{\infty} w(t)(Z / t) d t=\frac{2 n^{2} Z}{(n+1)(2 n+1) t_{c}} ; \quad \bar{t}=\int_{t_{c}}^{\infty} t w(t) d t=\frac{2 n^{2} t_{c}}{(n-1)(2 n-1)} .
$$

As discussed below, an unbiased sensing scheme will yield a Cross-Correlation Function (CCF) will allow the the duct-averaged velocity $\langle V\rangle$ to be calculated as a weighted integral (sum) ratio:

$$
\bar{v}=\int \operatorname{CCF}(t)(z / t)^{3} d t / \int \operatorname{CCF}(t)(z / t)^{2} d t
$$

for any transit time distribution $W(t) d t$ that can be represented as a sum of weighted delta-functions,

$$
W(t)=\sum m_{j} \delta(t-\pi,) .
$$

As discussed further on, this representation of $W(t)$ corresponds to a CCF composed of a similarly weighted set of response functions:

$$
\begin{array}{ll}
m_{j}^{\prime}=m_{j}, & t<\frac{Z}{v_{c}[1-a / R]^{1 / n}} \\
m_{j}^{\prime}=m_{j} \arcsin \frac{a / R}{1-\left(Z / V_{c} t\right)^{n}} ; & t>\frac{Z}{v_{c}[1-a / R]^{1 / n}} .
\end{array}
$$

The strip reading bias effect can be calculated by numerical integration; however, the readout error cannot be corrected by such a calculation as neither $V_{c}$ nor the index are known before a measurement is made.

The above applies to any type of strip-reading electrode geometry. The reading bias of the ANL Mk1 Sensing Capacitor (and similar geometries that enclose a circular duct between parallel plates) is further increased through 
the series capacity of the Teflon surround between duct wall and electrode surface. To estimate this effect, we shall neglect the difference in dielectric constant between duct wall and surround, as well as the curvature of fleld lines outside the duct. For this simplifled model, sketched in FIG (IV$7)$, the contribution $\Delta C(x)$ to the overall signal from the strip of width $\Delta x$ at $x$ is diminished, relative to its value $k_{m} \Delta x / 2 y$ in the absence of serles capacity, by the factor $F(x)=\Delta C_{m} / \Delta C_{s}$ :

$$
\Delta C(x)=\frac{x_{m} \Delta x}{2 y\{1+F(x)\}} ; \quad F(x)=\beta \frac{r-y(x)+\Delta h}{y(x)}, \quad \beta=\frac{x_{m}}{x_{s}} ;
$$

$\mathbf{R}_{\mathrm{s}}=$ dielectric constant of the surround,

$k_{m}=$ medium dielectric constant.

$\Delta h=$ distance between electrode and duct wall.

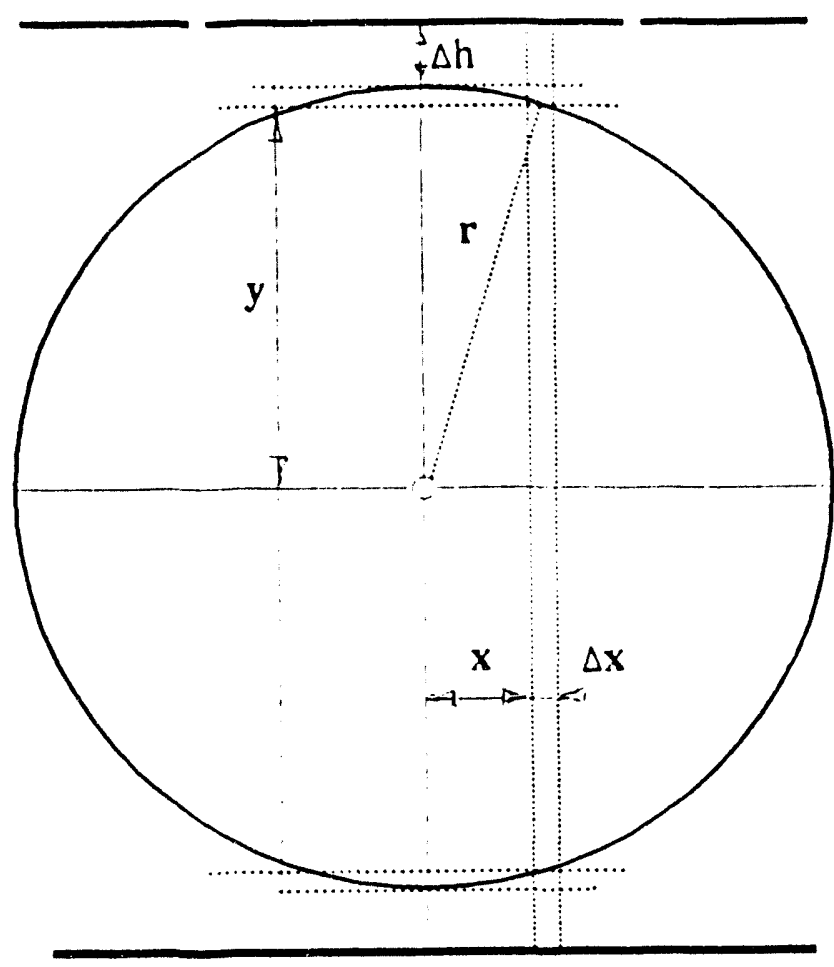

FIG (IV-5): Series Capacity associated with ANL Mk 1, schematic

As $\beta$ can be very large for aqueous slurries, the effect is certainly far from negligible. Were it possible to tinker with the Managan geometry, one might consider replacing the Teflon surround with one cast from a hightemperature epoxy loaded with a suitable mixture of alumina and barium titanate or titanium dioxide that matches the dielectric constant of the surround, hence, $\beta<1$ for coal slurries. One might then compensate for the bias effect by connecting the separate signal electrode strips recommended by the reference design study (say one central, $j=1$ and two lateral pairs, $j=2,3$ ) to different amplifiers with adjustable gains $A(j)$, such that, knowing the dielectric constant ratio $\beta$, 


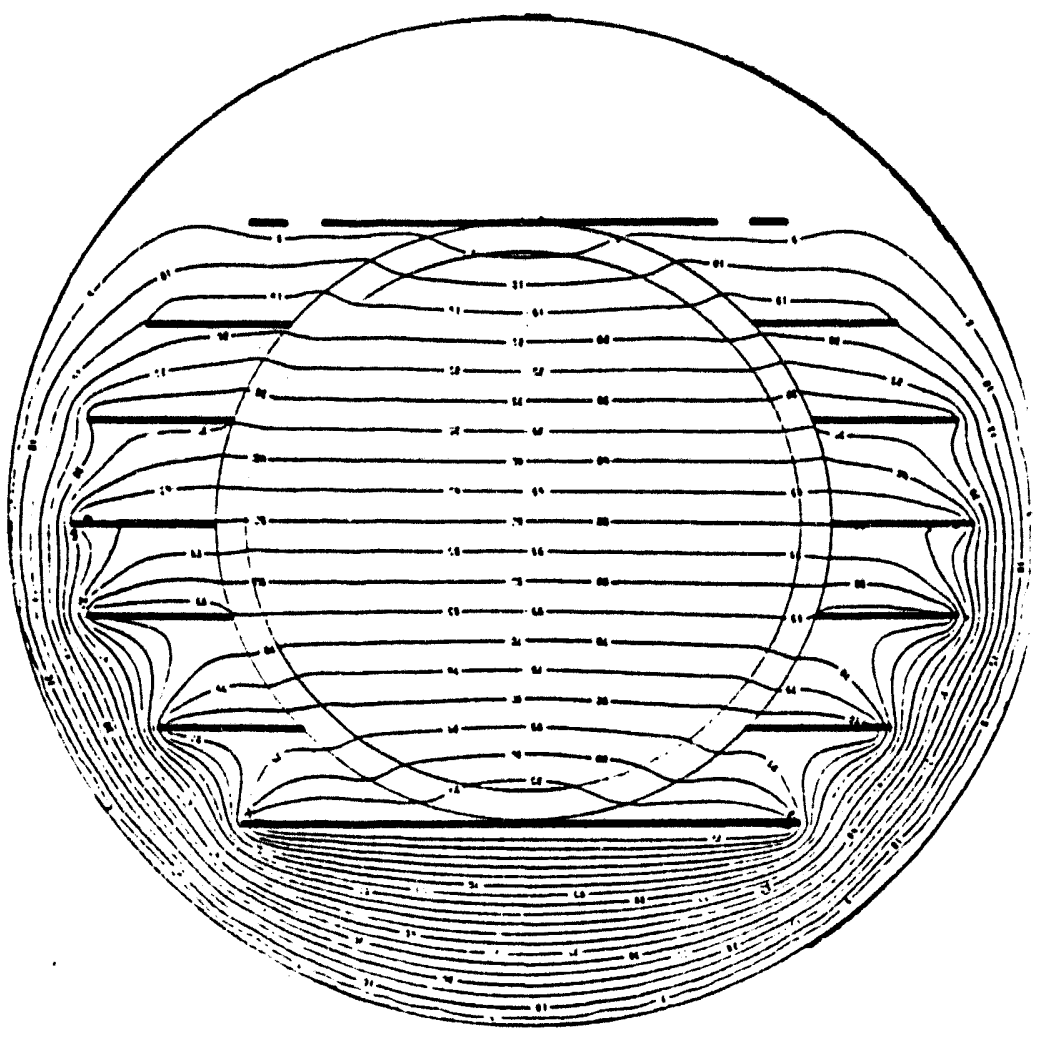

FIG(IV-6): Equipotentials, ANL Mk 1 , for $k_{m}=4.00$

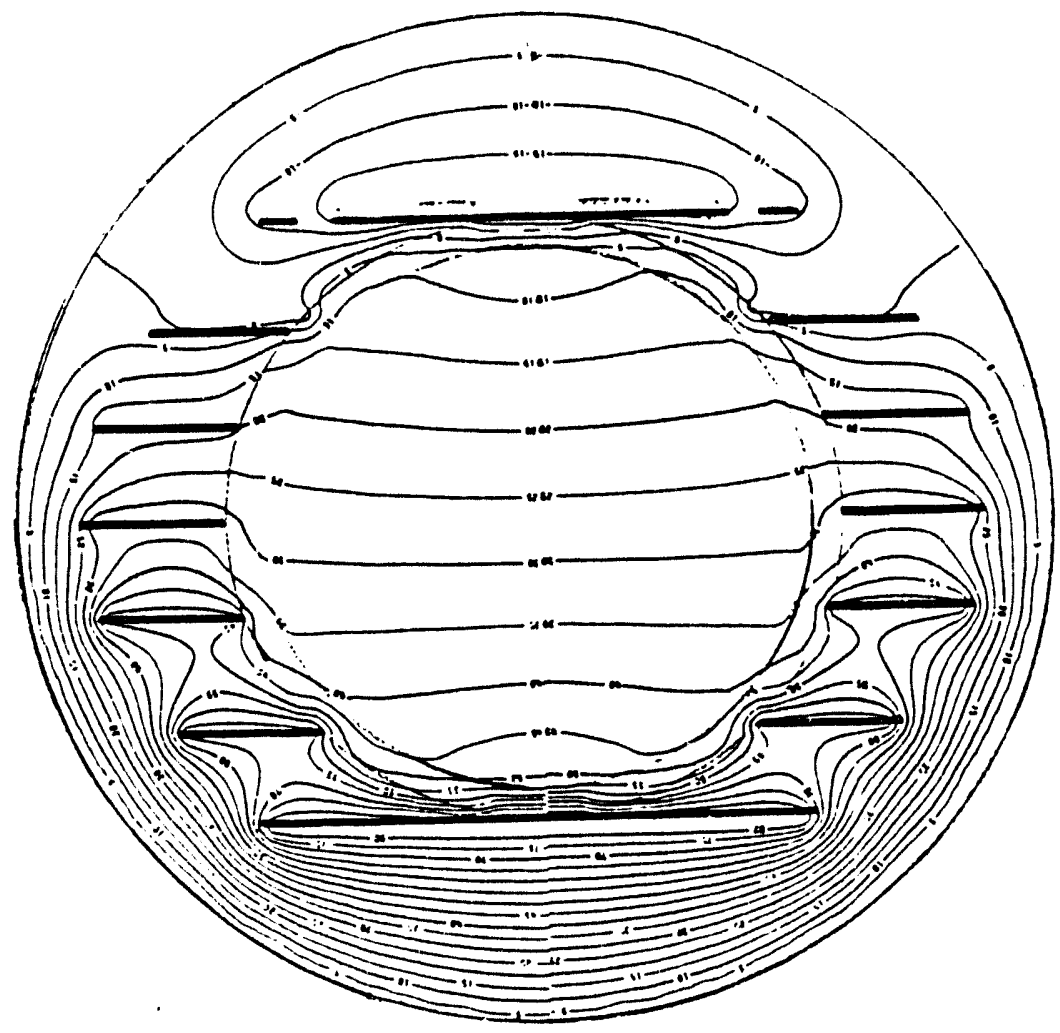

FIG(IV-7): ANL Mk 1 Equipotentials calculated for $k_{m}=60.00$ 


\section{$A(j)=1+\beta\left(1-F^{\prime}(J)\right)$}

where $F^{\prime}(j)$ is averaged for the electrode strip and/or Includes some compensation for finlte strip width bias. Alternatively, strip width bias could be corrected for from a second set of readings limited to the central electrode, requiring another complete set of CCF computations and yielding a narrowstrip/wide-strip ratio of apparent flow velocitles that could be interpreted in terms of the flow mode. While all this should not be difficult to implement, once the on-line computer is programmed to calculate the correction, it cannot be guaranteed to read the flow velocity within an acceptable error, say $1-2 \%$ (let alone an error level sultable for process control, $0.5-1 \%$ ).

\section{(b) Wrapped-Plate Electrodes}

This popular sensing geometry, shown in FIG (IV-8) in cross section, avolds the serles capacity problem of the ANL MkI type of scheme discussed in the preceding section. FIG(IV-8) was calculated by means of the WP*.FOR program set (cf. App.B), designed to match the "asymmetric" driving scheme of the Fenstermacher calculations $(E-2)$. With regard to duct interior coverage and field uniformity, it provides a tradeoff: As shown in the Figure, calculated for a wide-coverage design, the sensing field (indicated by equipotential line density) becomes very strong in the vicinity of the plate edges, both within and without the duct. For a narrow-electrode design, field uniformity improves

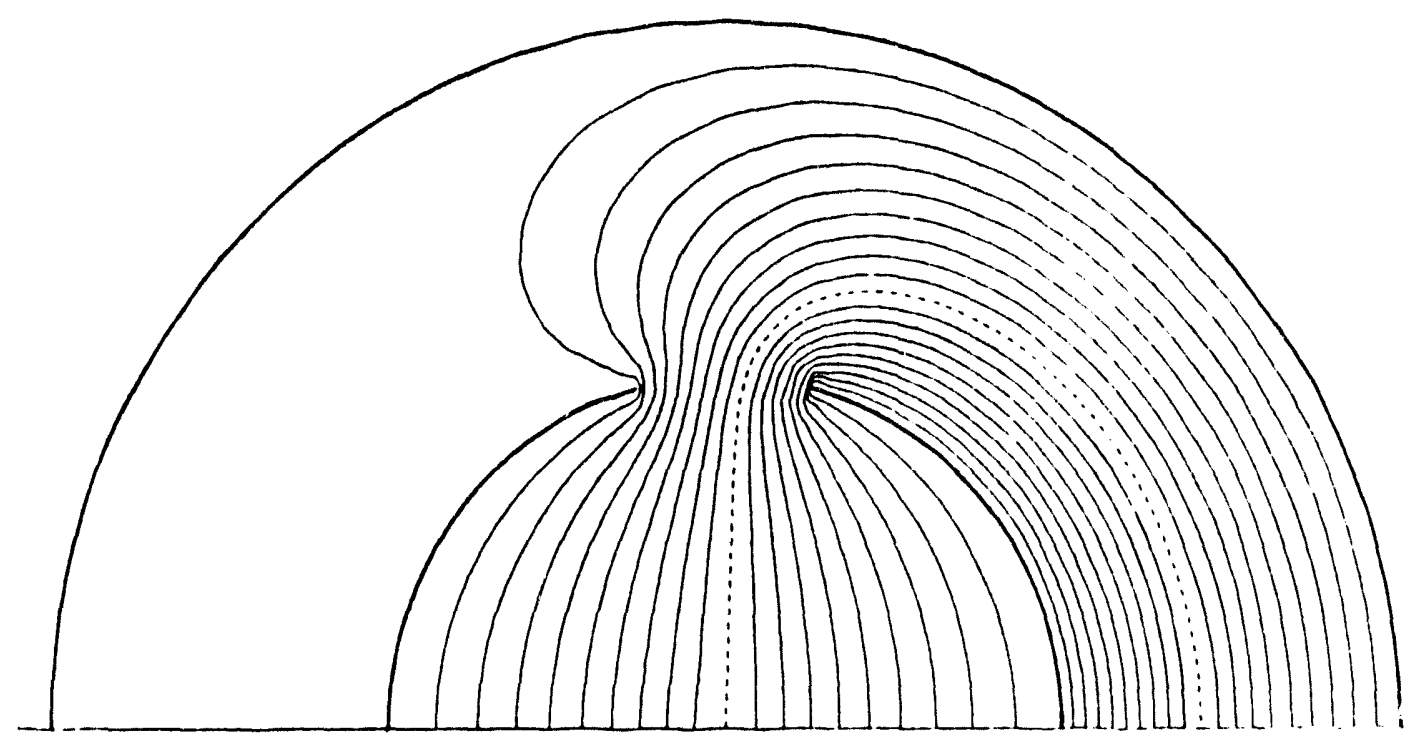

FIG(IV-8): Wrapped-Plate Geometry Equipotentials 
while coverage worsens. If contrasting-dielectric regions (duct wall) are factored in, it further develops that coverage as well as fjeld uniformity vary with the ratio of dielectric constants, hence, with the solid fraction of the medium. All this leads to the conclusion that this geometry, though widely u'sed, senses the duct with an unavoidable (as well as unpredictable) bias.

\section{(c) "Patch" Signal Electrude}

The sensing geometry is shown in FIG (IV-9). Equipotentials for this geometry have not been calculated but evidently resemble those of the preceding scheme in that the field tends to be much stronger in the vicinity of plate edges (and therefore produces a strong sensing bias favoring the peripheral region); it also senses, with some emphasis, the region along the edges of the patch and just outside the duct. The "patch" geometry, simple to construct, thus produces a large readout error.

\section{(d) Adjacent Ring Geometry.}

This geometry, proposed by (amongst others) Irons and Chang (111-10), is shown in FIG (III-10): another scheme that is simple to construct but produces a strong edge-field - sensing, together with especially strong sensing outside the duct. As before, one must expect the ratio of outside to inside sensing, to vary with the dielectric constant ratio. For these various reasons, this geometry cannot be recommended.

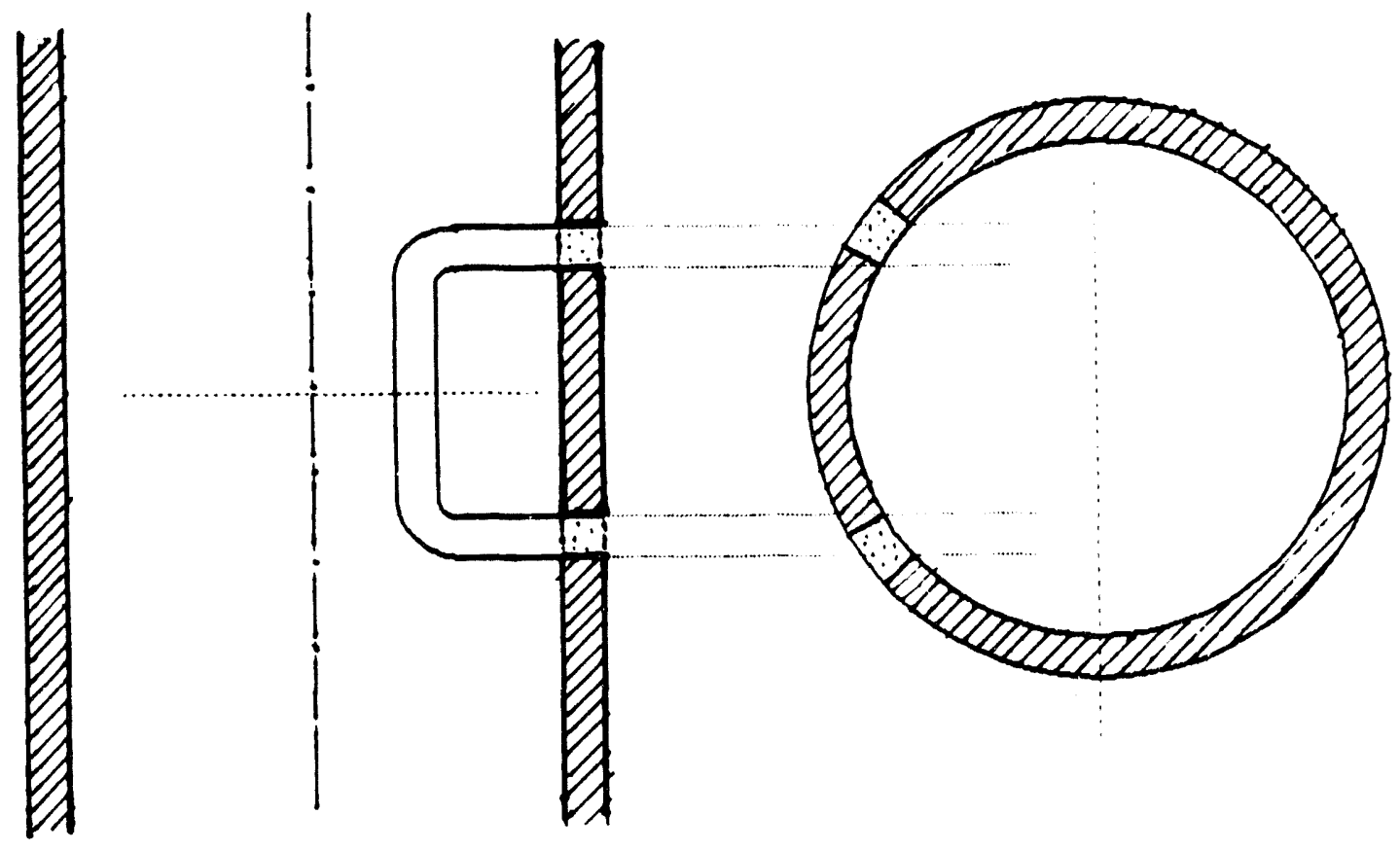

FIG(IV-9): "Patch" Electrode Geometry, schematic 


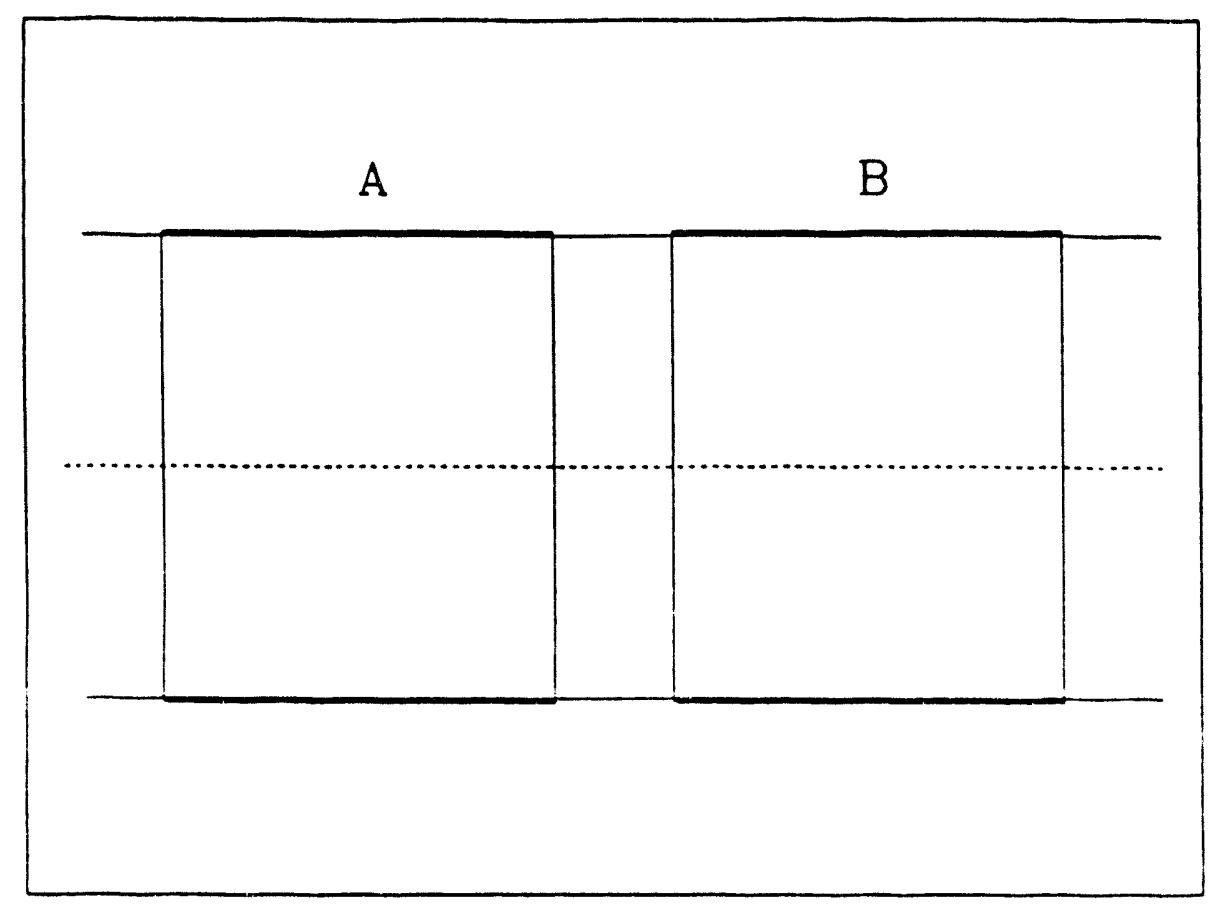

FIG(IV-10): "Adjacent-Ring" Electrode Geometry, schematic

(e) Helical Strip Electrodes.

This scheme, apparently reinvented a number of times(E-8)-(E-11), envisages partial phase separation in a horizontal duct and attempts to cancel, or reduce, the resultant bulk density readout error by rotating the sensing capacitor axis. The somewhat complex subject of phase stratification error is treated in refs. $(E-10)$ and $(E-11)$ and reviewed below. Because rotation schemes have been strongly promoted, it seemed worth while to calculate a set of equipotentials for this geometry. The spiral arrangement is shown in FIG (IV-11) and the equipotential plot, in FIG (IV-12). According to that figure, preferential sensing of the peripheral flow results from both a strong edge-field and an especially weak field average along the duct axis, where a series of zero-field saddle points are featured. Averaging the readout from capacitors of different orientation that sense a two-phase stratified or fully separate medium in a square duct is discussed further on. To the extent to which that exercise applies to stratified flow in a duct of circular cross section, it indicates that the deviation from the signal for uniformly mixed phases is negative for horizontal and positive for vertical plates; the magnitude of the deviation, however, is in the ratio of $2: 1$ hence, straight averaging (the equivalent of rotation) only reduces, but does not remove, stratification error. One must therefore conclude that spiral wrapping not only fails to sense unformly or sense exclusively within the duct, but also fails to deal effectively with stratification error, its ostensible rationale. 


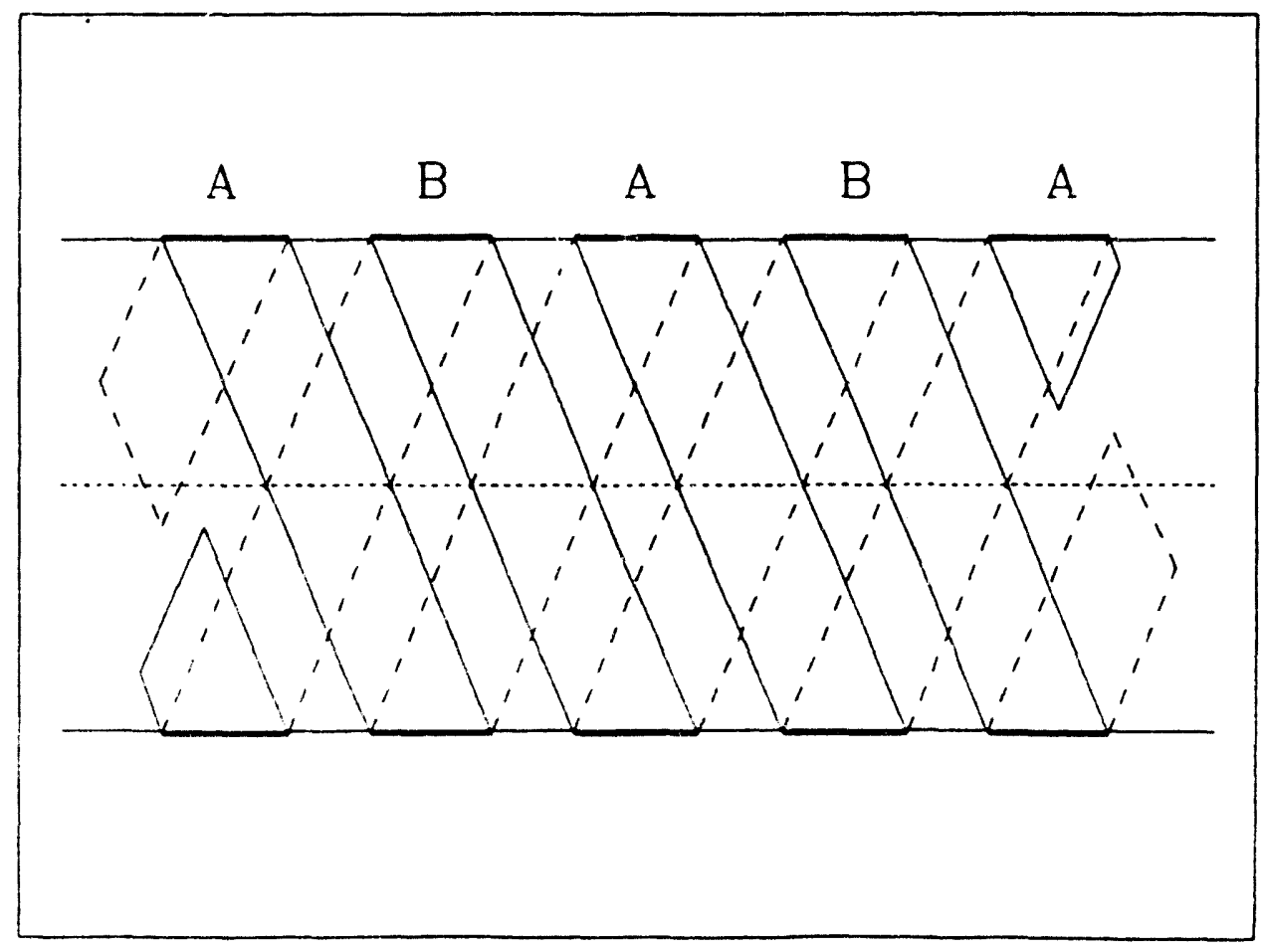

FIG(IV-11): Helical Strip Geometry, schematic

\section{(f) Rotating Field Scheme}

This scheme replaces a helical electrode arrangement with a set of six button or strip electrodes at equal distances around the inner duct wall. Three sensing fields are laid across the duct from the signal electrode to driven electrodes straight through the axis and at 30 and -30 deg from that direction, the other 2 electrodes are disconnected. Signal and driver electrodes are rotated at a suitable frequency; readings are summed. This arrangement at least avoids edge-fields and thus confines sensing to the duct interior, with fair sensing uniformity, somewhat biased toward the central region. As discussed below, rotation somewhat reduces the readout error resulting from stratification of the solids in a long horizontal duct. The system, evidently an improvement over those described earlier, has been patented and is offered commercially $(E-12)$.

\section{(g) Mixed-Geometry Scheme}

Another patented device(E-13), shown in FIG (III-13), features parallel plates outside the duct, enclosed by an outer cylindrical duct at a voltage one half of that of the driving electrode. A highly uniform field within the duct is claimed to result, however, the equipotential plot provided by the patentee is entirely inconsistent with Poisson's equation. This example is included here to caution readers of the patent literature as well as to emphasize the need for calculations instead of free-hand drawings. 


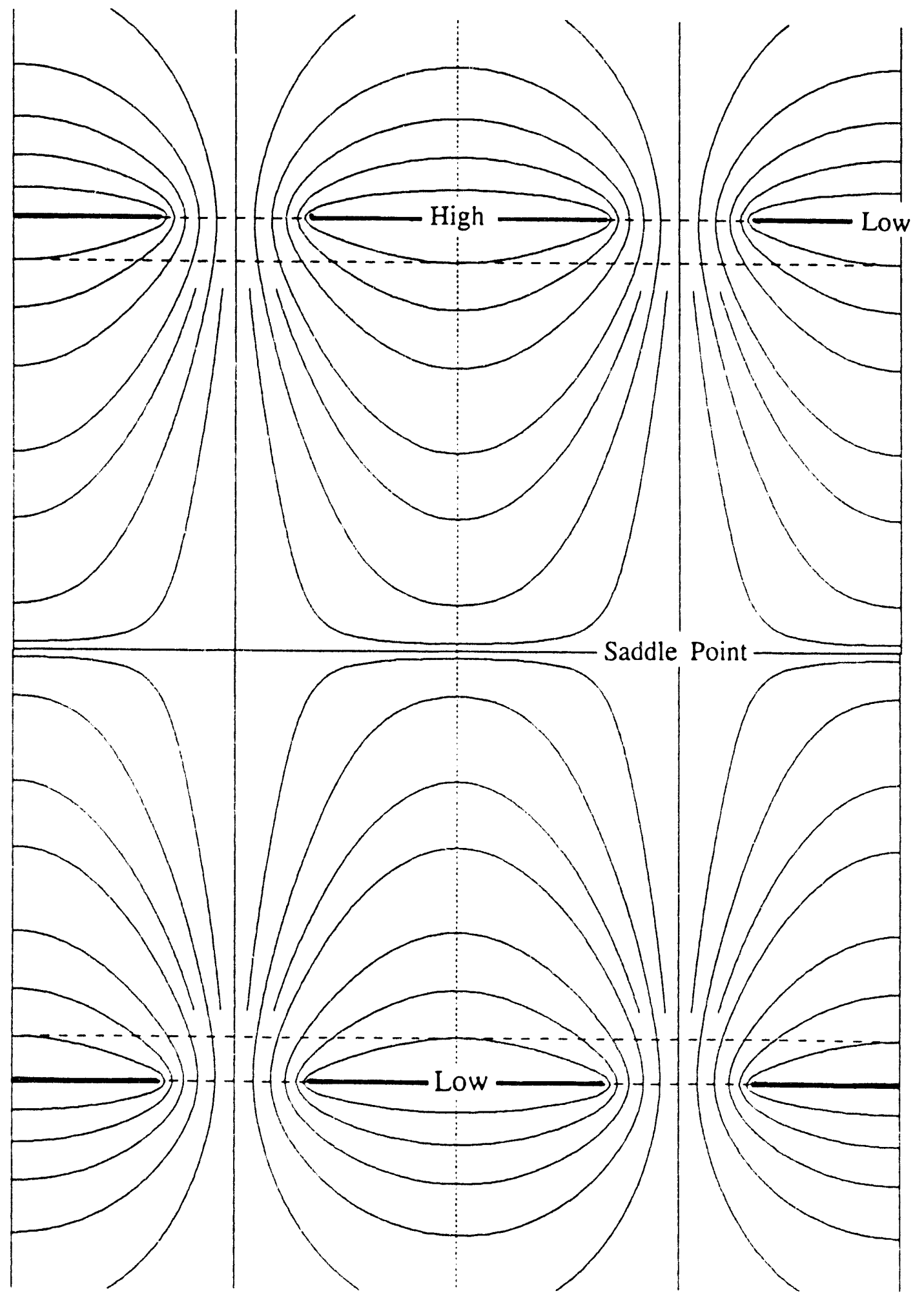

FIG(IV-12): Equipotentials for Helical Strip Electrode Geometry 


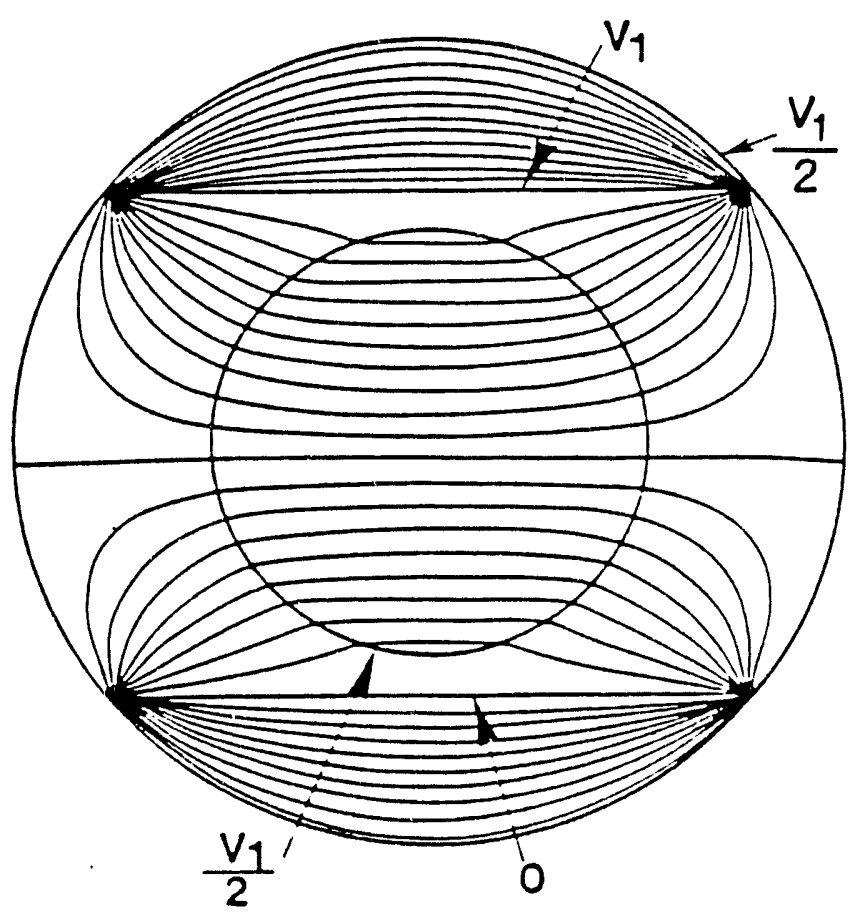

FIG(IV-13): Pseudo-Equipotentials for "Mixed" Geometry

\section{(h) ANL Spoolpiece \# 2 (Wrapped-Plate with Guard Strips)}

In the preceding discussion of the Wrapped-Plate scheme, it was noted that this design offers a certain tradeoff between Field (sensing) uniformity and coverage, both related to sensing/readout bias when medium composition (or, velocity) is non-uniform. While this tradeoff cannot be avoided altogether, it can be significantly improved through the use of Guard Strips. Guard Plates have been routinely used in parallel plate geometry for over 100 years. to define the edge of a sensed region. The Parallel-Plate ANL Mk 1 scheme is an example of this stratagem, as well as of the regulation of equipotentials by means of sets of parallel shims connected to an RC-chain Voltage divider.

If this scheme, designed for parallel-plates, is applied to a wrappedplate electrode pair (E-14) with grounded strip guards, adjacent to the signal electrode, forming the electrode equipotential edge, the signal does not "see" the strong edge-field and the duct is thus sensed with relative uniformity, while a region of the order of $5 \%$ of the duct escapes viewing. The geometry and a set of equipotentials are shown in FIG (IV-14) (calculations assume a uniform dielectric throughout; medium-wall dielectric contrast inevitably will have some effect on equipotential and field line shape, hence, the sensed duct fraction). Electrodes can be emplaced on the outside of an insulating duct or, preferably, form part of the duct wall, connected by dielectric strips and duct segments. The angle which the Guard strip makes with a line through the duct center is more or less arbitrary, the purpose of this extension being to prevent field lines that pass outside the duct from reaching the signal electrode. A prototype spoolpiece was tested in a number of runs on a gas-solids loop. Aithough using electronics that had inadequate bandwidth as well as a primitive cross-correlation computer, results obtained were encouraging(E-17). 


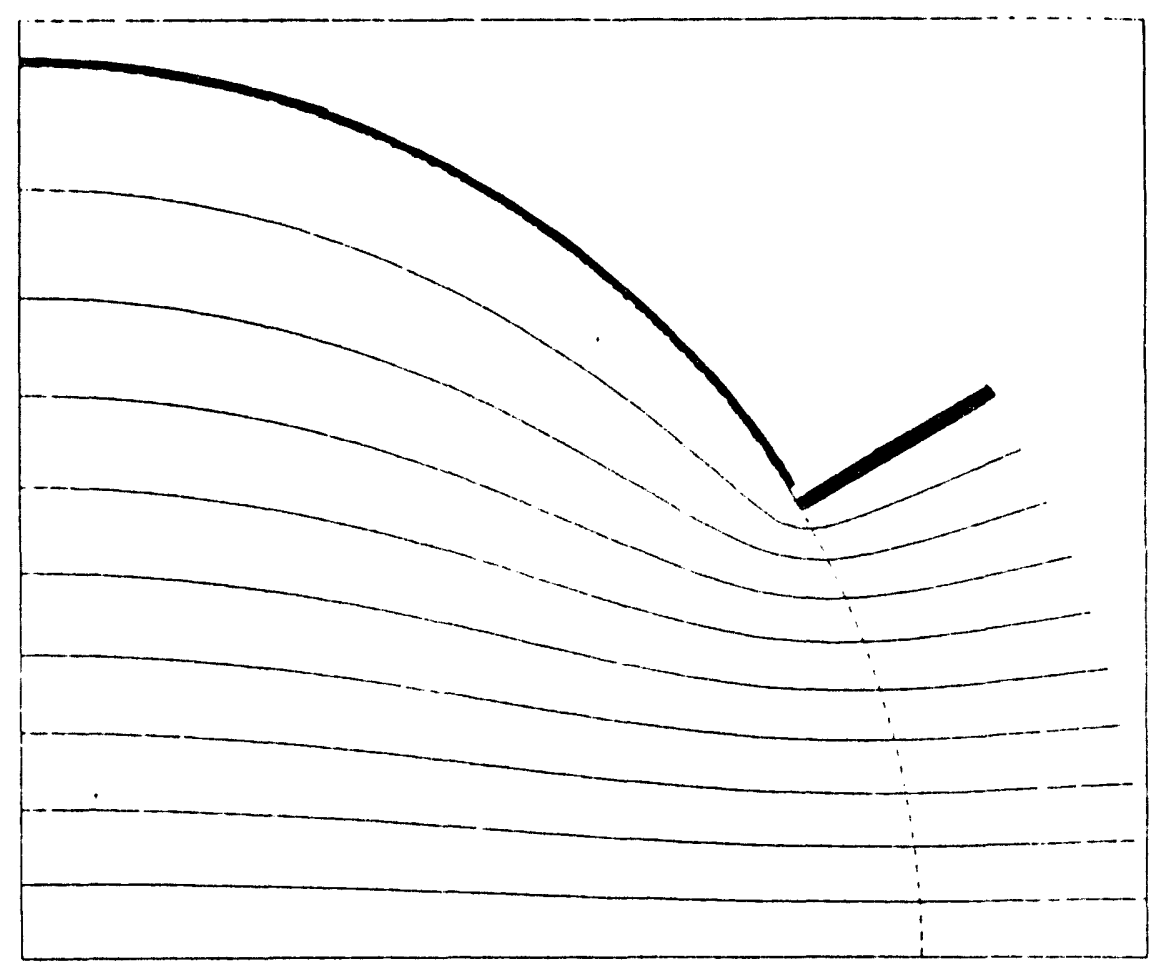

FIG(IV-14): ANL Mk 2 Geometry and Equipotentials

\section{(5) Local Field Strength and Sensing Efficiency}

In the foregoing, it was repeatedly asserted that the effect on the readout of a local inhomogeneity varies with the square of the local field strength. We now examine this proposition. Referring to an arbitrary set of equipotentials that connect a pair of electrodes, as sketched in FIG (IV-15), we label these $1,2, \ldots i \ldots n$ and single out the strip bordered by a pair of adjacent field lines, consisting of a string of cells of width $\Delta w_{1}$ and length $\Delta h_{1}$. The local field strength for constant $\Delta V$ comes to

$$
E_{1}=-\Delta V / \Delta h_{1},
$$

cell capacities (per unit length) are likewise constant,

$$
c_{1}=c=\mathrm{kx}_{0} \Delta \mathrm{w}_{1} / \Delta \mathrm{h}_{1}=\Delta \mathrm{q} / \Delta \mathrm{V}
$$

hence,

$$
E_{1} \Delta w_{1}=-\Delta V c / K_{0}
$$

is a constant as well. The cell volume $\Delta v_{1}=\Delta h_{1} \Delta w_{1}$, a variable, is inversely 


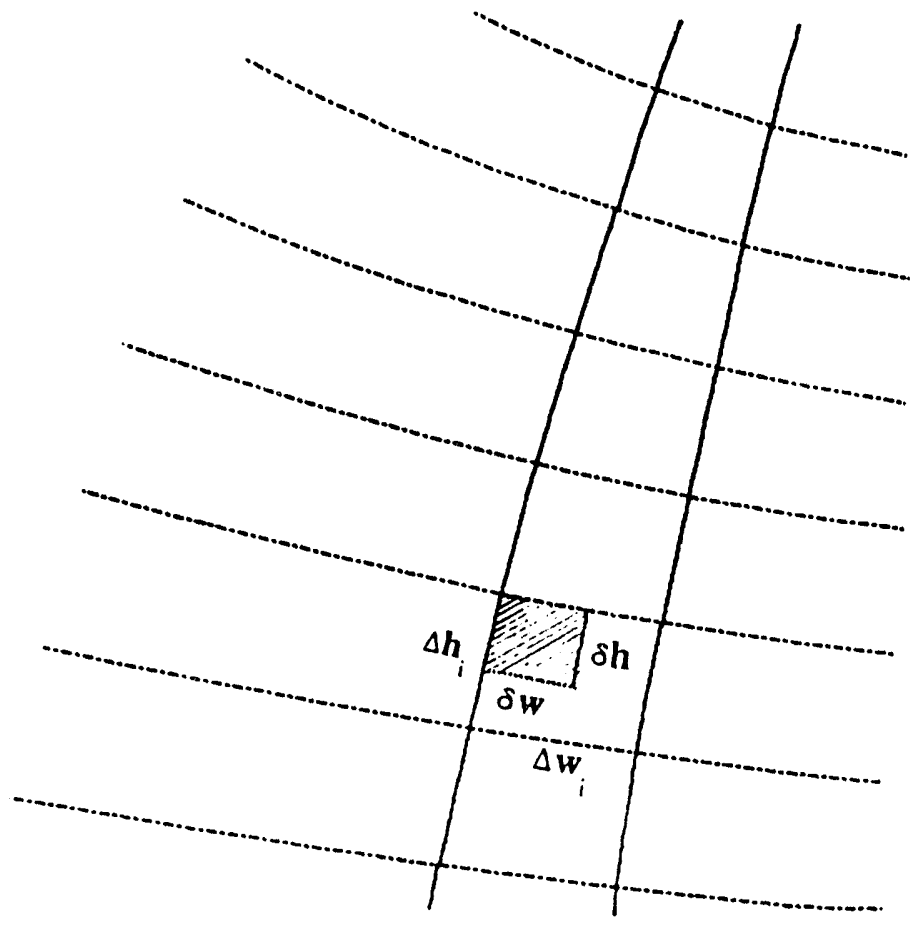

FIG(IV-15): "Cell" Contribution to the Signal, schematic

proportional to the square of the local field strength:

$$
\Delta v_{1}=\Delta h_{1} \Delta w_{1}=c \Delta v^{2} / k_{0} E_{1}^{2} \text {. }
$$

We now introduce a sub-region of width $\delta \mathrm{w}$, height $\delta \mathrm{h}$, volume $\delta \mathrm{v}$ and dielectric constant $\mathrm{k}^{*}$ into cell $\mathrm{i}$, altering the local capacity by $\delta c_{1}$ :

$$
\begin{gathered}
\delta c_{1}=c_{1}^{*}-c=\frac{c f \delta v}{\Delta v_{1}\left(1-f \delta h / \Delta h_{1}\right)}=\frac{f \delta v k x_{0} E_{1}^{2}}{\Delta v^{2}\left(1-f \delta h / \Delta h_{1}\right)} ; \\
f=\left(k^{*}-k\right) / k^{*} .
\end{gathered}
$$

The insertion modifies the capacity $C$ contributed to the readout signal by the entire strip; adding cell capacities in series,

$$
\frac{1}{\mathrm{C}}=\sum_{i=1}^{n} \frac{1}{c_{1}}=\frac{n}{c} ; \quad \frac{1}{c^{*}}-\frac{1}{c}=\left(\frac{1}{c_{i}^{*}}-\frac{1}{c_{1}}\right)
$$

hence, putting $\delta C=C^{*}-C, C / C=1 / n$, such that

$$
\frac{\delta C}{C}=\frac{\delta c_{1}}{c} /\left[n+(n-1) \frac{\delta c_{1}}{c}\right]
$$

and introducing the combined constant 


$$
g=f \delta v k R_{0} / c \Delta v^{2}
$$

one obtains the relationship between the signal effect $\delta C / C$ of the local insertion of an altered region, and the local Field Strength $E_{1}$ :

$$
\frac{\Delta C}{C}=\frac{g E_{1}^{2} / n}{1+f \delta h E_{1} / \Delta V+(n-1) g E_{1}^{2} / n}
$$

Q.E.D.

\section{(6) The Bulk Dielectric Constant of Binary Mixtures}

Although a number of engineering reports and manufacturer's brochures that deal with capacitive densitometry seem to assume that the measured capacity or signal varies linearly with medium composition (phase fraction), that assumption is, at best, a poor approximation and at worst, misleading. Even for highly uniform, molecular mixtures, the Clausius-Mosotti law (E-18), a generally excellent approximation discussed from a modern point of view by refs. (E-19) and (E-20), predicts a non-1inear relationship: for a set of polarizabilities $\alpha_{k}$ and molecular number densities $n_{k}$,

$$
F=\frac{1}{3 k_{0}} \sum \alpha_{k} n_{k}=\frac{H_{0}}{H_{o}+3}
$$

or

$$
H_{0}=\frac{3 F}{1-F}
$$

$$
\begin{aligned}
& \mathrm{x}_{0}=8.85 \mathrm{pf} / \mathrm{m}, \\
& \mathrm{H}_{0}=\mathrm{k}-1,
\end{aligned}
$$$$
\mathbf{K}=\text { mixture dielectric constant }=\mathrm{C}_{\mathrm{m}} / \mathrm{C}_{\mathrm{o}} \text {, }
$$

$\mathrm{C}_{0}=$ reference measurement with an empty capacitor,

$\mathrm{C}_{\mathrm{m}}=$ measured capacity with molecular mixture medium present.

For a mixture of two components, say, a mixture of two alcohols, Eqn. IV24 yields

$$
\frac{k_{m}-1}{k_{m}+2}=\phi_{1} \frac{k_{1}-1}{k_{1}+2}+\left(1-\phi_{1}\right) \frac{k_{2}-1}{k_{2}+2}
$$

hence,

$$
H=\frac{h \phi_{1}}{1+h k_{2}\left(1-\phi_{1}\right) /\left(\mathrm{k}_{2}+2\right)}
$$

where 


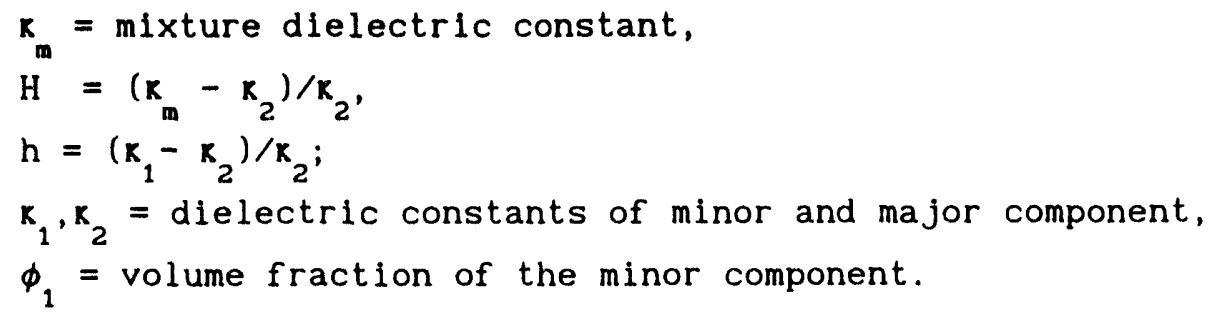

For a suspension of droplets or particles in a gas $\left(k_{2}=1\right)$,

$$
H=\frac{h \phi_{1}}{1+h\left(1-\phi_{1}\right) / 3} .
$$

Note that Eqn. IV-27 is reversible (invariant with exchange of indices); note further that the relation between the "signal" $H$ and $\phi_{1}$ is not linear.

Binary mixtures on a scale well beyond molecular (dispersions of particles or bubbles in a fluid) require a different treatment. A number of relations, agreeing in second order but diverging in third order, between the bulk dielectric constant and the dispersed component phase fraction have been derived, based on different models. Because the assumption of linearity is widely held by instrumentation engineers (mirabile dictu. "linear" measurements have been reported), a simple derivation of the oldest of these equations, due to Maxwell, is presented below.

In FIG (IV-17), parallel plates produce a uniform field $\mathbb{E}$ within a large region filled with dielectric 2 . N spherical dielectric 1 particles of radius a are randomly embedded in a spherical sub-region of radius $A$, centered at the origin. The potential at $(O, R)$ can be calculated in two ways: first, considering sphere $A$ to have an effective or bulk dielectric constant $k$,

$$
\begin{aligned}
& V=-\mathbb{E}\left[R-f A^{3} / R^{2}\right] ; \\
& f=\left(k-k_{2}\right) /\left(k+2 k_{2}\right),
\end{aligned}
$$

secondly, as the superposition of potentials due to the $\mathrm{N}$ individual spheres. One particular such sphere, centered at $\left(r_{1}, \vartheta_{1}\right)$ where the potential comes to

$$
V\left(r_{1}, \vartheta_{1}\right)=-\mathbb{E} r_{1} \cos \vartheta_{1} \text {, }
$$

contributes the effect

$$
\Delta V_{1}=-\mathbb{E} s_{1} \cos \Theta_{1}\left[1-\mathrm{ma}^{3} / \mathrm{s}_{1}^{2}\right],
$$$$
\cos \Theta_{1}=\left(R-r_{1} \cos \vartheta_{1}\right) / s_{1} ; \quad s_{1}=\sqrt{R^{2}+r_{1}^{2}-2 R r_{1} \cos \vartheta_{1}} ;
$$

hence, putting $x_{1}=r_{1} / R, \mu_{1}=\cos \vartheta_{1}, m=\left(k_{1}-k_{2}\right) /\left(k_{1}+2 x_{2}\right)$, 


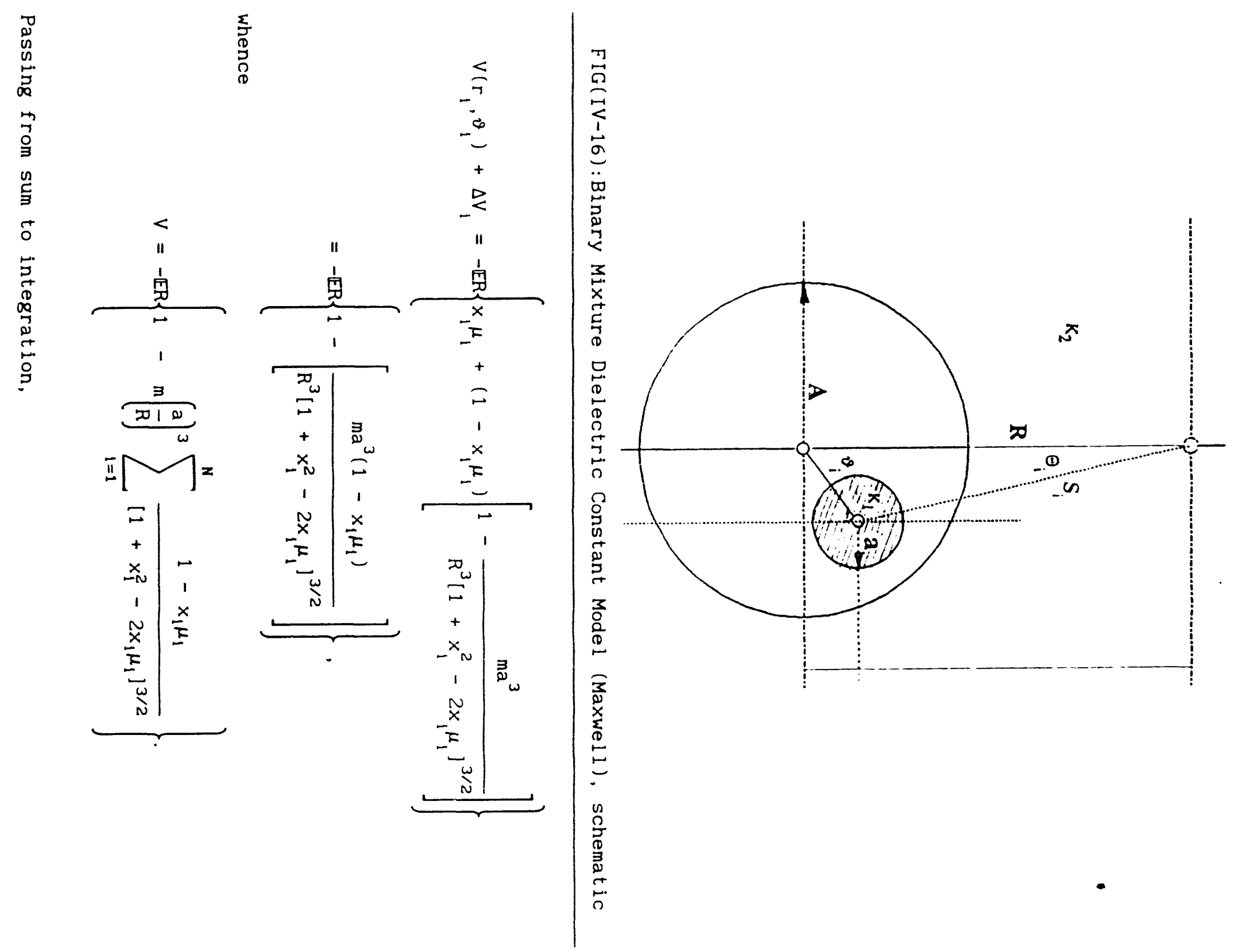




$$
V=-\mathbb{E R}+\frac{3 \mathbb{E R m a}}{2 A^{3}} \int_{0}^{A / R} x^{2} d x \int_{-1}^{+1} d \mu\left[\frac{(1-x \mu)}{\left(1+x^{2}-2 \mu x\right)^{3 / 2}}\right]=-\mathbb{E}\left[R-m \phi_{1} A^{3} / R^{2}\right], \quad(I V-29)
$$

where we have put

$$
\phi_{1}=N(a / A)^{3}
$$

Comparing $(C-12)$ and $(C-13)$, one finds that $M=m \phi_{1}$, or, explicitly,

$$
\frac{R_{m}-K_{2}}{R_{m}+2 k_{2}}=\phi_{1} \frac{R_{1}-R_{2}}{R_{1}+2 R_{2}} ;
$$

rearranging and introducing $H$ and $h$ (defined above), one obtains the MaxwellRayleigh second-order signal/composition relation

$$
H=\frac{h \phi_{1}}{1+h\left(1-\phi_{1}\right) / 3}
$$

originally given by Maxwell (E-21). The derivation reproduced above does not allow for the effect of particles on the local field and therefore is slightly incorrect to second order (and more so to higher orders) in $\phi$; it is also nonreversible (as one would expect with a model specifically introducing spheres 1 suspended in a continuum 2 ).

Reconsidering the problem, Rayleigh(E-22) introduced a regular array of spheres throughout the region and derived an equation that adds a third-order correction term to Eqn. IV-30a, subsequently corrected by Runge(E-23) as well as Meredith and Tobias (E-24); as the third-order term already turns out to be rather complex, fourth-order correction is impractical. Investigations by Molyneux(E-24) and Brown(E-26) suggest that the inherent error in a regular lattice model (relative to a random particle dispersion) may be of third order. All this suggests that the Maxwell-Rayleigh formula is not accurate beyond second order and thus should be applied (if at all) only to dilute suspensions.

A considerably different treatment of this problem, by Bruggeman(E-26), led to the relation

$$
(1+H) h^{3}\left(1-\phi_{1}\right)^{3}=(h-H)^{3} .
$$

This equation is reversible. Expanding and dropping terms of higher than second order,

$$
H=3 h \phi_{1}\left\{3+h-\phi_{1} h\left[1+\frac{h}{3+h}\right]\right\}^{-1} \text {. }
$$

Making judicious use of a more accurate version of the Clausius-Mosotti equation, Bottcher(E-20) suggested the relation

$$
H /(1+H)=3 h \phi_{1} /(h+3+2 H)
$$


which, rearranged and reduced to second order, comes to

$$
H=3 h \phi_{1}\left\{3+h-\phi_{1} h\left[1+\frac{2 h}{3+h}\right]\right\}^{-1}
$$

Landauer(E-27), seeking a formula for the conductivity of binary alloys, a mathematically similar problem, found and expression that turns out to be just another version of Bottcher's relation, Eqn. IV-33. Note that the secondorder approximations (Eqns. IV-30a, IV-32 and IV-34) differ only by small factors of second order in both $\phi$ and $h$. The more accurate Eqns. IV -31 and IV-33 are both reversible.

Looyenga(E-28) and independently, Landau and Lifschitz(E-29), once again reexamining the binary mixture problem, proposed the (reversible) relation

$$
(H+1)^{1 / 3}-1=\phi_{1}\left[(h+1)^{1 / 3}-1\right] .
$$

A comparison of these formulae to available measurements(E-19) shows that Eqn. IV-35 fits the data somewhat better than Eqns. IV-31 and IV-33 and considerably better than Eqn. IV-30a; it is therefore adopted in this report. Since values of $\phi$ up to 0.6 are not uncommon in slurry feedlines, the full equation rather than a second-order approximation is appropriate: let

then,

$$
g=(h+1)^{1 / 3}-1
$$

$$
H=3 g \phi\left(1+g \phi+g^{2} \phi^{2} / 3\right)
$$

expresses the relation between signal and composition. To calculate $\phi$ from the measurement $H$, the on-line computer must solve the equation

$$
\phi=(H / 3 g) /\left[1+g \phi+(g \phi)^{2} / 3\right],
$$

for which an iterative scheme is practical. Eqn. IV-37 is also useful in determining the intrinsic dielectric constant of a specific variety of a generic solid such as coal: aliquots are first ground and combined according to wellestablished sampling principles; a sample of the averaged material is finely ground and compressed to a pill that fits within a small parallel-plate test capacitor. Weighing and pycnometry determine the pill solid fraction $\phi$; the capacitor measures the bulk dielectric constant $k_{m} ; k_{1}$ is calculated from

$$
k_{1} \phi_{1}^{3}=\left[k_{m}-3 k_{m}^{1 / 3} \phi_{2}\left[k_{m}^{1 / 3}-\phi_{2}\right]-\phi_{2}^{3}\right] \text {. }
$$

The plots shown in FIGS (IV-17) and (IV-18) illustrate the predictions of the major formulae reviewed above for coal-oll and coal-water slurries with varying solid fraction. To scale these plots, the linear equation $H(1$ in $)=h \phi$ was subtracted from each bulk dielectric constant parameter $\mathrm{H}$, calculated from Eqns. IV-27 (Molecular Mixture), IV-30a (Maxwel1-Rayleigh), IV-31 (Bruggemann), IV-33 (Bottcher) and IV-35 (Looyenga and Landau). The "Molecular Mixture" for- 


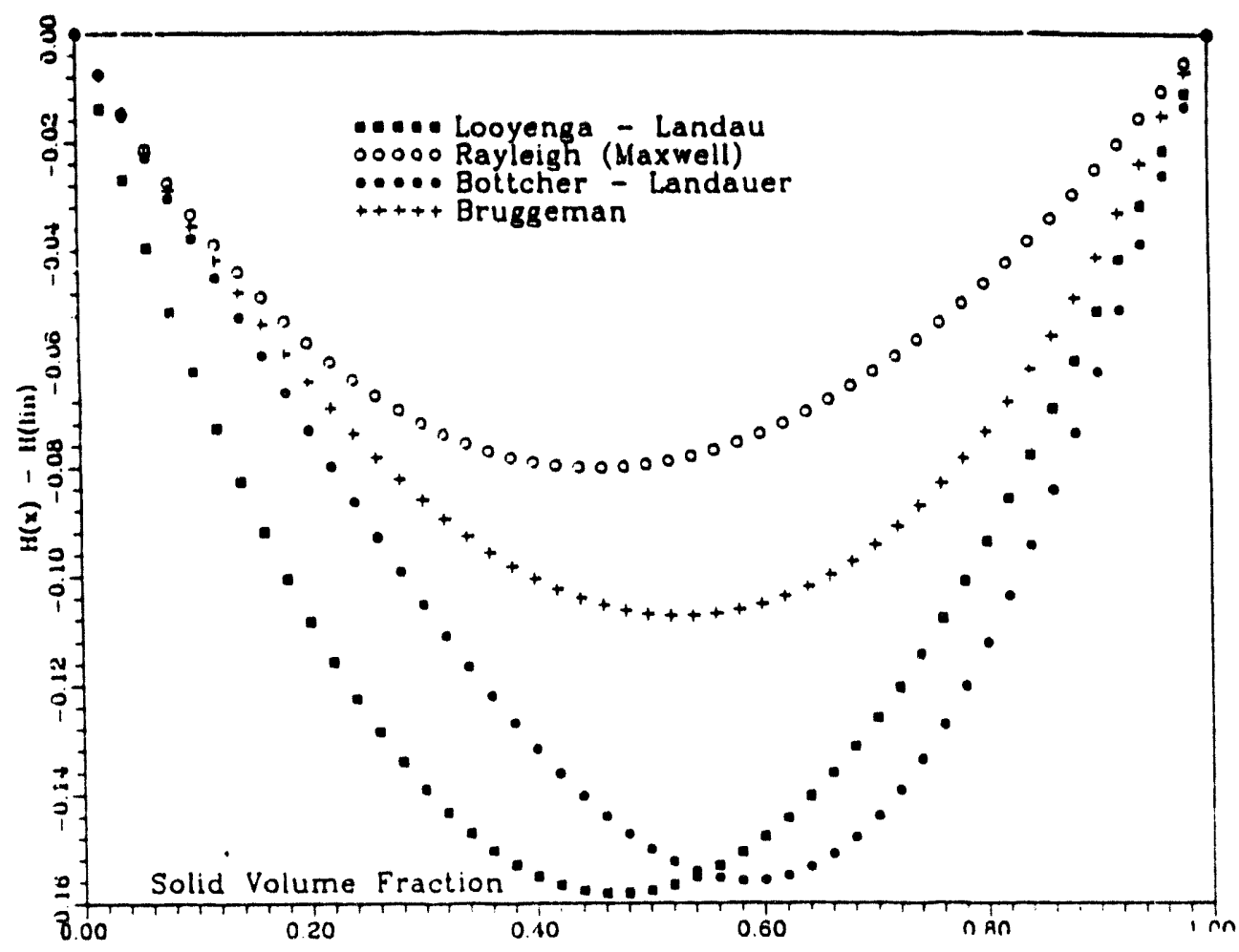

FIG $(I V-17): k_{m}$ Non-Linearity vs. $\phi_{s}$ for different Models: $k_{s}=6.0, k_{f}=60$.

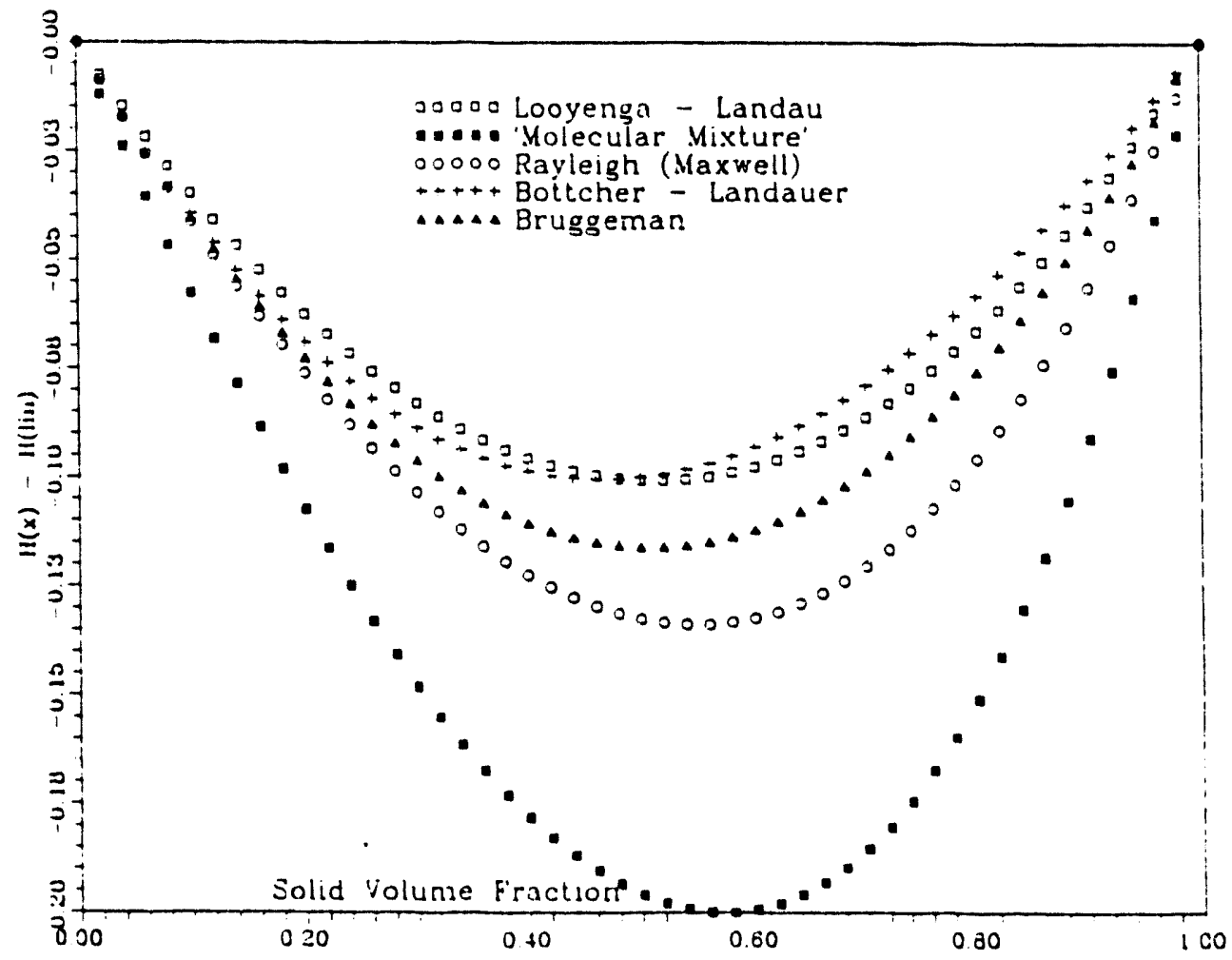

FIG(IV-18): $k_{m}$ Non-Linearity vs. $\phi_{s}$ for different Models: $k_{s}=6.0, k_{f}=2.5$. 
mula, not really pertinent to a binary mixture, was plotted only for the coaloll case, where it evidently deviates from the remaining formulae. Note that realistic solld fractions do not exceed 0.6 ; note further that the Looyenga formula has shown the clostest agreement with careful measurements; note, finally, that according to that formula, non-linear (second and higher order) terms amount to $10 \%$ for coal-o1l, $16 \%$ for coal-water, over the range of solid volume fractions expected in slurry feedlines.

\section{(7) Effect of a Phase Gradient}

As discussed in the preceding chapter, much attention has been focused on the tendency of entrained solids to settle as the slurry flows in a long horizontal duct at or below the "critical" velocity. In fact, that problem hardly exists at the extreme densitles mandated by considerations of economy for Chemical plant feedline conveyors. Phase separation must certainly be considered in connection with pipelines - by the engineer who designs the conveyor. In a well-designed pipeline, one would expect a somewhat stratified medium - vell above critical velocity yet not entirely uniform and thus yielding a certain readout error. The elimination, or reduction, of this phase non-uniformity error has been the ostensible objective of EM sensing designs with rotating or spirally wound electrode configurations, discussed in the preceding. An alternative, simpler scheme will be considered here: vertically and horizontally oriented sets of plates are emplaced on the duct and individually read out; readings are then combined with optimal weights.

To investigate this setup, it is expedient to consider a measurement section with a square cross section of sidelength $a$, eliminating field nonuniformity and similar issues and concentrating the discussion on the effects of phase stratification or separation. We further require a phase distribution model: for simplicity, we assume here a two-layer distribution that considers solids either uniformly distributed throughout the duct, or settled (in a uniform distribution) in a bottom layer whose height $h$ is determined by an assumed limiting solid volume fraction $\Phi=0.6$, while the top layer of helight a-h is assumed to be pure fluid. This somewhat extreme but not perhaps entirely unrealistic model is illustrated in FIG (IV-19).

For a vertical plate set, the layer system defines two parallel capacitors whose capacities per unit length of duct come to

$$
C_{1}=C_{0} K_{H} \beta, \quad C_{2}=C_{0} K_{f}(1-\beta), \quad \beta=h / a ;
$$

from Eq. (IV-36),

$$
\mathrm{k}_{\mathrm{m}}=\mathrm{k}_{\mathrm{f}}(1+\mathrm{g} \Phi)^{3}
$$

substitution in Eq. (IV-39) and summing yields

$$
C_{v}=C_{1}+C_{2}=C_{f}\left[1-B+B(1+g \Phi)^{3}\right]
$$

With the same amount of solids uniformly distributed throughout the duct, the capacity comes to 

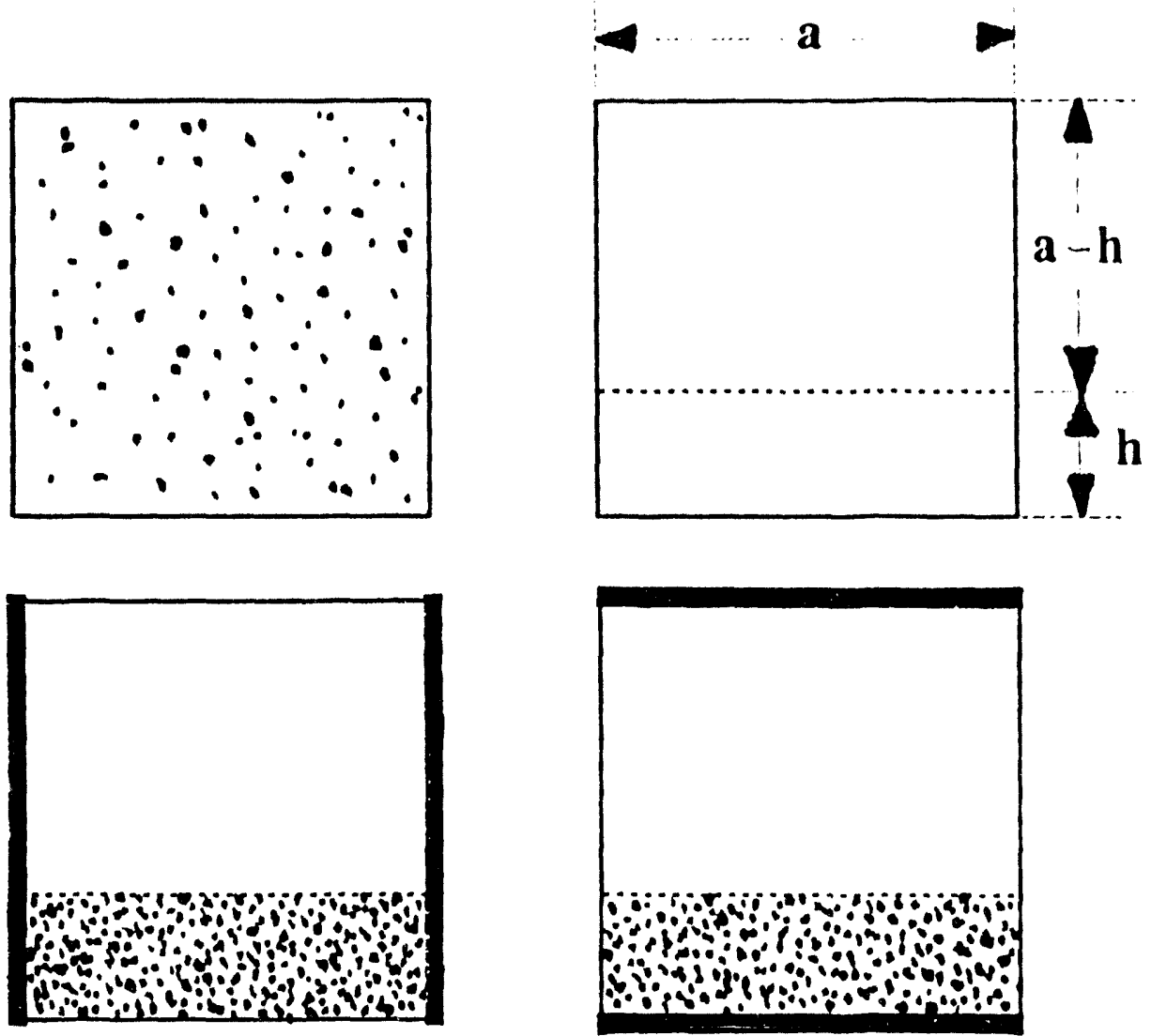

FIG (IV-19): Layered Phase Separation Model, schematic

where

$$
C^{*}=C_{f}[1+g\langle\phi\rangle]^{3}
$$

$$
\langle\phi\rangle=B \Phi .
$$

$C$ * may be considered the "true" reading. Let $\alpha=g\langle\phi\rangle$ and put $k=(1-\beta) / \beta$,

$$
R^{*}=C^{*} / C_{f}-1, \quad R_{v}=C_{v} / C_{f}-1, \quad R_{h}=C_{h} / C_{f}-1
$$

The relative readout error $\varepsilon_{v}=R_{v} / R^{*}-1$ can be written in those terms as

$$
\varepsilon_{v}=\alpha k \frac{1+\alpha(k+2) / 3}{1+\alpha+\alpha^{2} / 3}
$$

or, expanded in a series,

$$
\varepsilon_{v}=\alpha k\left[1+\alpha(k-1) / 3-\alpha^{2} k / 3 \ldots\right]
$$

Next, for a horizontal plate set, the layer capacities add in series, 


$$
C_{h}=C_{f} \frac{(1+\alpha / \beta)^{3}}{B+(1-\beta)(1+\alpha / \beta)^{3}} \text {, }
$$

hence, after a similar treatment, one obtains the fractional readout error

$$
\begin{aligned}
& \varepsilon_{h}=\left(\varepsilon_{v}-k R_{v}\right) /\left(1+k R_{v}\right) \\
& =k \alpha[-2+10(k-1) \alpha / 3 \ldots] .
\end{aligned}
$$

Comparing Eqns. IV -43 and IV-45, one finds that vertical plate sensing (VPS) makes a positive, horizontal sensing (HPS) a negative first-order error - however, the HPS error is twice the VPS error. Thus, the direct or unwelghted average

$$
\langle C\rangle_{u}=\left(C_{v}+C_{h}\right) / 2
$$

makes a first-order error

$$
\varepsilon_{u}=\left(\varepsilon_{h}+\varepsilon_{v}\right) / 2=-k \alpha / 2+11 k(k-1) \alpha^{2} / 6 \ldots
$$

that can be reduced to second order by introducing weights $q$ to combine the two readouts in the weighted average

$$
\langle C\rangle_{w}=\left[q C_{v}+C_{h}\right] /(1+q) ;
$$

in particular, the weight choice $q=2$ eliminates the first-order error:

$$
\varepsilon_{w}=4 k(k-1) \alpha^{2} / 3 \text {. }
$$

Optimum weights vary with both solid volume fraction and dielectric constants, as illustrated in FIGS (IV-20)-(IV-21). Note that the coal-oil slurry illustration indicates a worst-case weighted error of about $0.5 \%$ for dense slurries $(\phi \cong 0.6)$, increasing to $-2 \%$ for the unweighted case.

The foregoing elementary model assumes electrodes that fully cover duct sides. For a more realistic comparison to circular duct geometry, electrodes that do not extend to the duct corners must be assumed. This leads to a more complex error equation for vertical plates but does not change the horizontal plate error; depending on parameter choices (especially, $\beta$ ), the vertical plate error can be slightly larger, or slightly smaller than Eqn. IV-43. A more realistic phase stratification model, featuring e.g. an exponential density gradient, likewise ylelds more complex expressions, without revealing new insights; admitting that the foregoing model is somewhat oversimplified, it does allow the general conclusion that weighted-average sensing and readout can enhance accuracy relative to straight averaging by "rotation".

To implement such a scheme requires separate horizontal and vertical plate sets with separate channel electronics; the weighted average is produced by computer programming. In addition to the weighted sum, it may be useful to compute and indicate the difference, proportional to the degree of phase stratification (which could provide warning of incipient problems and/or support fine-tuning of the conveyor). 


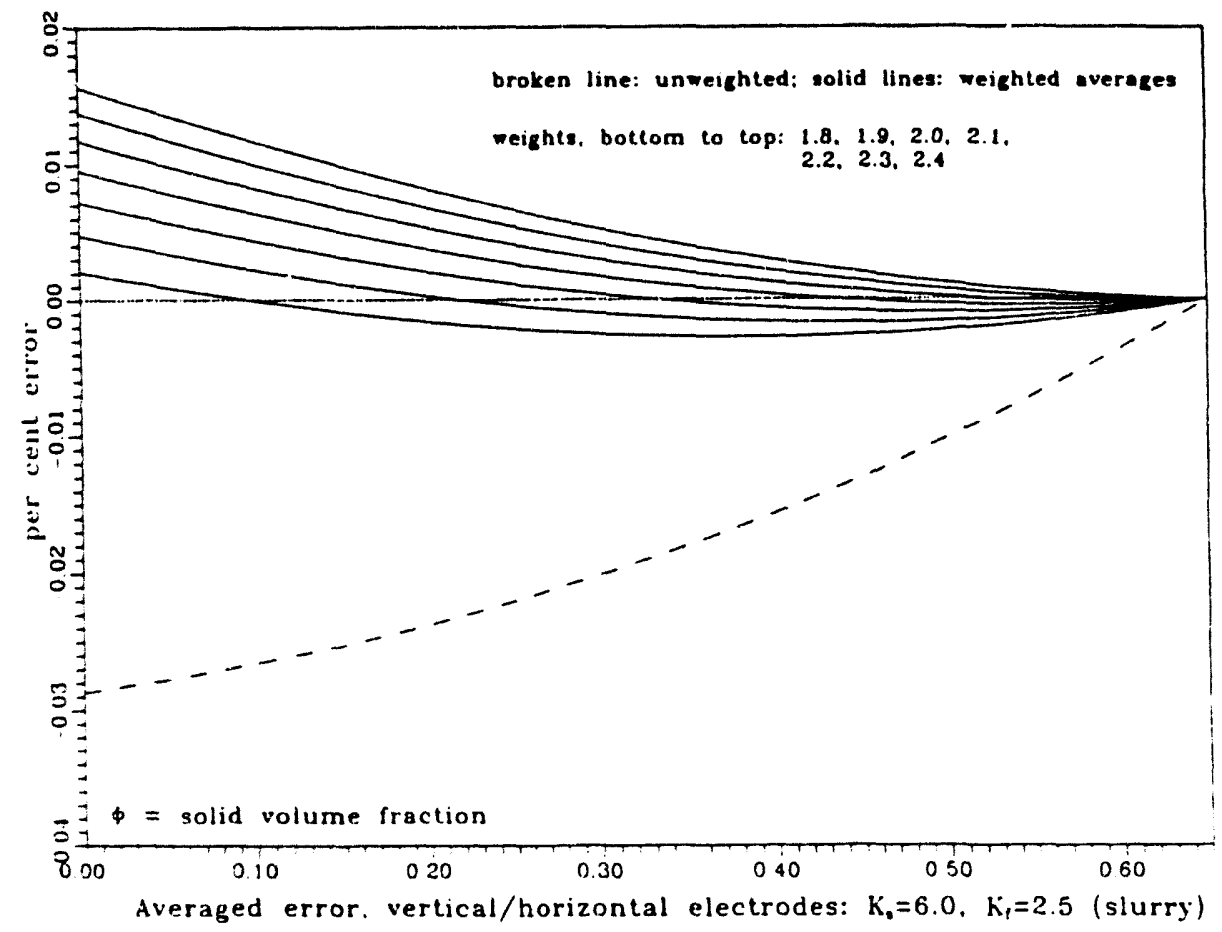

FIG (IV-20): Bulk Density Readout Error for coal/oil slurry, weighted and unweighted average of Horizontal and Vertical Plate sets.

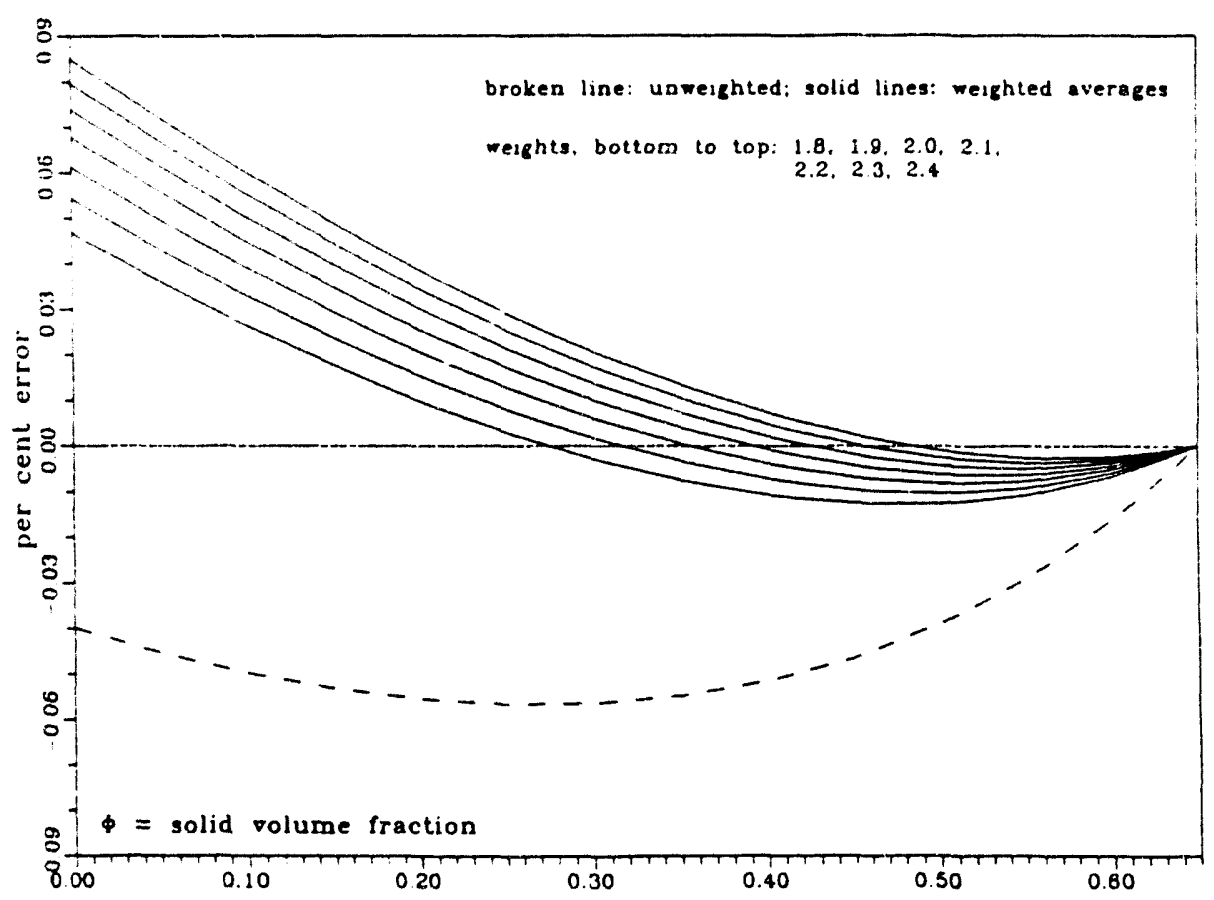

FIG (IV-21): Bulk Density Readout Error for coal/water slurry, weighted and unweighted average of Horizontal and Vertical Plate sets 


\section{(D) Stat,istics associated with Solid Fraction Fluctuations}

The solid fraction within the field of view of a detector installed on a flowing two-phase medium is expected to fluctuate in time, a phenomenon that, on one hand, allows the application of the Cross Correlation velocity measurement scheme discussed further on, and, on the other hand, results in an of $f$ set error of any measurement of the average Solid Fraction, if and when the device response to the Phase Fraction is non-1inear. Since that is indeed the case for both gamma transmission and EM sensing, this offset error is discussed here, for both sensing schemes.

\section{(1) Gamma Transmission}

That Gamma Transmission through a fluctuating absorber invoives an offset error has been noted, for instance, by Harms(E-30); the error is of same lype? as the Cross-Beam Phase Separation Effect, discussed above, and inviludes the (non-off set.l Statistical Error (treated in the foregoing). As before, ail Bernoulli selections with constant probabilities are combined into a parametor $\eta$; the transmission probability through the moving slurry. P. comes to

$$
P_{1}=e^{\left.-A_{1}\langle\phi\rangle+\Delta \phi_{1}\right]}=e^{-A<\phi\rangle}\left[1-A \Delta \phi_{i}+A_{i}^{2} \Delta \phi_{i}^{2} / 2 \ldots\right] .
$$

Let

$N_{1}=$ number of photons emitted towards the detector in count interval i., $\eta_{1} N_{1}=M_{1}=$ photons that are counted if transmitted by the medium.

$C_{1}=M_{1} P_{1}=$ counted photon number, all for the ith counting interval;

numbers $M$ and transmission $P$ are connected through

and

$$
W\left(M_{1}\right)=e^{-\langle M\rangle}\langle M\rangle^{M_{1}} / M_{1} !
$$

$$
B\left(M_{1}, k\right)=M_{1} !\left(P^{k} / k !\right)(1-P)^{M_{1}-k} /\left(M_{1}-k\right) !,
$$

the former being the probability that exactly $M_{1}$ photons are emitted by the source and selected by other processes, given the average number $\langle M\rangle$, the latter the probability that exactly $k$ out of $M_{1}$ photons are transmitted by the medium. Summing over $k$ from 0 to $M_{1}$ and $M_{1}$ from 0 to $\infty$,

$$
\langle C\rangle=\sum W\left(M_{1}\right) \sum k B\left(M_{1}, k\right) ;\left\langle C^{2}\right\rangle=\sum W\left(M_{1}\right) \sum\left[k(k-1)+k \mid B\left(k, M_{1}\right)\right. \text {; }
$$

hence, putting $\langle M\rangle e^{-\lambda<\phi}=\left\langle C_{0}\right\rangle$, the count for a stationary medium,

$$
\begin{aligned}
\langle\mathrm{C}\rangle & =\left\langle\mathrm{C}_{0}\right\rangle\left[1+\mathrm{A}^{2} \operatorname{var}(\phi) / 2\right] ; \\
\left\langle\mathrm{C}^{2}\right\rangle & =\left\langle\mathrm{C}_{0}\right\rangle^{2}\left[1+2 \mathrm{~A}^{2} \operatorname{var}(\phi)\right]+\langle\mathrm{C}\rangle ;
\end{aligned}
$$




$$
\operatorname{var}(C)=\left[\left\langle C_{0}\right\rangle^{2}+\left\langle C_{0}\right\rangle / 2\right] A^{2} \operatorname{var}(\phi)+\operatorname{var}\left(C_{0}\right)
$$

The second term of the count variance results from Poisson gamma source fluctuations; the first term, vanishing for a stationary medium, represents the uncertainty contributed by absorber fluctuations. These fluctuations also produce an of $f$ set in the mean $\langle\mathrm{C}\rangle$.

\section{(2) EM Field Sensing}

Turning now to EM Field sensing, let

$$
\begin{aligned}
& E_{m}=\text { voltage signal from sensor viewing the medium, } \\
& E_{r}=\text { pure fluid reference signal, }
\end{aligned}
$$

then the Looyenga relation can be evaluated in terms of moments $\mu_{j}(\phi)$. If electric noise, etc. can be neglected, the average readout comes to

$$
\left\langle E_{m}>/ E_{f}-1=(1+\alpha)^{3}-1+3 g^{2} \mu_{2}(1+\alpha)+g^{3} \mu_{3}\right.
$$

where $\alpha=g\langle\phi\rangle$, indicating an offset $3 g^{2} \mu_{2}[1+\alpha]+g^{3} \mu_{3}$ (which cannot be corrected for unless moments $\mu_{2}, \mu_{3}$ of the phase distribution are known). To find the variance, note that

$$
\begin{aligned}
& \left\langle E_{m}^{2}\right\rangle=E_{f}^{2}\left\langle(1+g \phi)^{6}\right\rangle \\
& =E_{f}^{2}\left\{(1+\alpha)^{6}+15 g^{2} \mu_{2}(1+\alpha)^{4}+20 g^{3} \mu_{3}(1+\alpha)^{3}+15 g^{4} \mu_{4}(1+\alpha) \ldots\right\} ; \\
& \left\langle E_{m}>^{2}=E_{f}^{2}\left\{(1+\alpha)^{6}+6 g^{3} \mu_{2}(1+\alpha)^{4}+2 g^{3} \mu_{3}(1+\alpha)^{3}+9 g^{4} \mu_{2}^{2}(1+\alpha)^{4}\right.\right. \\
& \left.+6 g^{5} \mu_{3} \mu_{2}(1+\alpha) \ldots\right\} ;
\end{aligned}
$$

hence,

$$
\operatorname{var}\left(E_{m}\right) / E_{f}^{2}=9 g^{2} \mu_{2}(1+\alpha)^{4}+18 g^{3} \mu_{3}(1+\alpha)^{3}+3(1+\alpha)\left(5 \mu_{4}-3 \mu_{2}^{2}\right) .(I V-54)
$$

Neglecting $\mu_{3}, \mu_{4}$ etc,

$$
\begin{aligned}
& \left\langle E_{m}>/ E_{f}=(1+\alpha)^{3}+3 g^{2}(1+\alpha) \operatorname{var}(\phi),\right. \\
& \operatorname{var}\left(E_{m}\right) / E_{f}^{2} \cong 9 g^{2} \operatorname{var}(\phi)(1+\alpha)^{4} ;
\end{aligned}
$$

as was the case for gamma transmission, offset and variance of the result are proportional to the phase fluctuation variance for EM sensing.

\section{(3) Error Removal through Short Sampling}

To correct for the offset, one could attempt to make an independent measurement of the phase fluctuation intensity. This turns out to be difficult as well as time-consuming; instead, it is expedient to linearize the readout by programming the dedicated computer to linearize the readout, using its 
arithmetic function repertoire or, should that be too slow, a Table Lookup and Interpolate operation. The sampling (counting) interval is chosen short enough to keep $\phi$ effectively constant during the measurement. For gamma transmission, logarithms of these short counts are computed and stored in an accumulatorconfigured memory; every few seconds, the accumulated sum of logarithms is divided by the sample number:

$$
\begin{aligned}
\langle R\rangle= & \frac{1}{N} \sum \log \left[\left[\langle\mathrm{C}\rangle+\Delta \mathrm{C}_{\mathrm{k}}\right] \mathrm{e}^{-\mathrm{A}\left[\langle\phi\rangle+\Delta \phi_{k}\right]}\right] ; \\
& \sum \log \langle\mathrm{C}\rangle+\sum \log \left[1+\Delta \mathrm{C}_{\mathrm{k}} /\langle\mathrm{C}\rangle\right]=\mathrm{N} \log \langle\mathrm{C}\rangle+\sum\left[\Delta \mathrm{C}_{\mathrm{k}} /\langle\mathrm{C}\rangle-0.5\left[\Delta \mathrm{C}_{\mathrm{k}} /\langle\mathrm{C}\rangle\right]^{2} \ldots\right] \\
= & \text { Nlog }\langle\mathrm{C}\rangle-1 / 2\langle\mathrm{C}\rangle+1 / 3\langle\mathrm{C}\rangle 2 \ldots \text { for Poisson fluctuations } \Delta \mathrm{C}_{\mathrm{k}} \text {. Thus, } \\
\langle\mathrm{R}\rangle= & \frac{1}{\mathrm{~N}} \sum\left[\mathrm{N} \log \left(\langle\mathrm{C}\rangle-1 / 2\langle\mathrm{C}\rangle-\mathrm{A}\left[\langle\phi\rangle+\Delta \phi_{k}\right]\right]=\log \langle\mathrm{C}\rangle-\mathrm{A}\langle\phi\rangle-1 / 2 \mathrm{~N}\langle\mathrm{C}\rangle,(\mathrm{IV}-55)\right.
\end{aligned}
$$

a direct measurement of the average phase fraction. A count $N\langle C\rangle$ of a few thousand makes the Poisson error $1 / 2 \mathrm{~N}\langle\mathrm{C}\rangle$ negligible.

For EM sensing, one must similarly take, and store, voltage signal samples by integrating over intervals during which the phases are constant, i.e., a time interval short in comparison to the expected transit time of solid particles through the detector station, $\delta t=\Delta z / V$ where $\Delta z=$ capacitor plate length, $V=$ median solids velocity:

$$
\langle\mathrm{R}\rangle=\frac{1}{N E_{\mathrm{f}}} \sum E_{m k}=[1+g\langle\phi\rangle]^{3}
$$

It may be noted that Phase fluctuations can be generated by pumping: certain types of pumps tend to produce strong clusters, appearing at the detector site at pump period intervals, which suggests a policy of counting over at least one pump period, preferably over several. In denser slurries, fluctuations are strongly suppressed and thus unlikely to be a source of error.

To sum up, phase fluctuation error, of ten overlooked and formerly difficult to deal with, can be managed by a data acquisition time strategy that can be implemented with an on-1ine PC.

\section{(4) Flow/No Flow Readout}

The dependence of mean and variance on phase fluctuations, developed in the foregoing for gamma transmission as well as EM Field sensing, can be exploited in a type of readout that is sometimes useful for purposes of warning. For example, in certain reactions, interruption of the solid feed shifts the equilibrium to a more exothermic mode, causing a rapid rise of the temperature that can result in considerable damage. Consequently, that type of plant is usually run in a conservative rather than maximum-efficiency mode. A fast, reliable Flow-No Flow indication could make efficiency attainable at an acceptable risk. 
The disappearance of $\operatorname{var}(\phi)$ as flow stops can be exploited in such an instrument: referring to Eqs. (IV-51) - (IV-54), a gamma transmission monitor whose readout is configured to compute the mean and second moment (a "Campbelling" channel, widely used in Nuclear Reactor instrumentation) and subtract the former from the latter will read zero if and when the flow stops. Similarly, a second moment-computing EM sensing channel will fall to the electronic noise level when the flow stops. The scheme was verified in tests on a vertical downflow feeder line(E-31).

\section{$\checkmark$ MEASUREMENT OF THE BULK DENSITY}

\section{(A) Instrument Design Strategies}

In this Chapter, binary mixture fluid mechanics and sensing scheme specifics that were reviewed in the preceding are applied to the design of densitometers, emphasizing two specific scenarios, chemical reactor slurry feed lines and pipelines, that appear to need better instrumentation.

The superior accuracy available from square duct EM sensing configurations has been pointed out; the difficulty of conventional instrument calibration in the specific applications selected here also has been put into the foreground. Yet, a large industrial plant may provide an on-site test loop and laboratory that makes calibration a feasible chore; square ducts may be considered inadmissible as long as their reliability has not been exhaustively tested. These considerations suggest a classification in terms of different Design Strategies:

(1) Conventional (Circular Duct \& Calibration),

(2) Intermediate (Circular Duct, Self-Calibrating),

(3) Optimized (Square Duct, Self-Calibrating).

This classification, intended only as a framework for the following discussion, is not meant to exclude any possible combination of strategies in an actual installation.

\section{(1) Conventional: Circular Duct, Calibration}

The conventional calibration of on-line densitometers relies on grabsampling. For dilute slurries, direct weighing of a standard container filled to the mark with a grab-sample taken directly from the feed line, together with a determination of the intrinsic densities of components, yields a reading of the solid fraction that can be accurate within < $1 \%$. Repetition with slurry of different makeup can provide a reliable calibration of a densitometer of almost any principle and configuration.

However, for a dense slurry medium at high pressure and temperature whose physical properties may change appreciably when cooled, grab-sampling is considerably less accurate or convenient than laboratory calibration consisting of two steps, bulk density measurement and EM device response measurement, re- 
peated for a number of representative samples at the pressure and temperature of the measurement site. Solid and fluid samples are weighed out and combined; aliquots are introduced into benchtop units designed for each task. Of a variety of schemes used for bulk density determination(D-4), (D-5), those involving floats, buoyancy indicators etc. are not applicable to slurries. Inertial response-vibrating U-tube devices can be designed for elevated temperatures but not pressures. A pycnometer scheme that can work at high pressure and temperature could be based on a container of known volume, equipped with a "chimney" and designed to withstand high pressures; an amount of the made-up slurry that rises into the neck at ambient is introduced; the container is sealed, heated and and pressurized with a Chemically inert hydraulic fluid. The interface between fluid and slurry is read with an acoustic echo device mounted on top of the chimney and equipped with a reference reflector bar.

The rest of the made-up sample is poured into a test loop consisting of a vertical piece of duct on which a replica of the EM sensing device is mounted; the remainder of the loop may be short and mainly of small diameter, as pumping only serves the purpose of agitation. Once again the medium is heated and pressurized with a hydraulic piston while the EM device is read. Both steps are repeated with a number of samples, so as to establish a calibration curve.

Instead of a separate pycnometric bulk density reading as described above, a more convenient, faster and probably more accurate calibration of the E.M. Densitometer can be provided by mounting a Gamma Densitometer on the loop. As discussed in Chapter IV, conversion of the Gamma Densitometer readout. to phase fraction requires a certain amount of information concerning the composition of solid and fluid, available from an Elemental or "Ultimate" and "Proximate" Analysis of the solid and formula of the fluid, together with accurate values of the intrinsic solids density. Of all this information, only the intrinsic solid density may not be available if the solid is coal. Density is not included in the ASTM information package on the basis of which coal is traded, nor is there an ASTM standard density measurement technique: as coal is microporous, different techniques can yield different "intrinsic" density values, as further discussed in section (B).

A bench-top test loop with EM and Gamma Densitometer is sketched in FIG (V-1) (omitting heaters, thermal insulation as well as the special pressure envelope that would be needed for $100 \mathrm{~atm})$. The Figure shows an ANL Mk 2 EM Densitometer. While it is true that any electrode geometry whatsoever can be calibrated, it seems reasonable to prefer those that restrict sensing to the duct interior, with a fairly uniform sensing field: in other words, something like the ANL Mk 2 geometry.

This describes Conventional Phase Fraction measurement (by Calibration). Needing neither Equations nor an on-line Reference measurement, this scheme is evidently limited to a fairly narrow range of slurry compositions. Since the significant variable in EM sensing is the bulk dielectric constant, calibration must certainly be repeated every time there is reason to believe that the dielectric constant of the solid feedstock has changed - this would be the case when a different type of coal (or, mixture of coal of different origin) is used. 


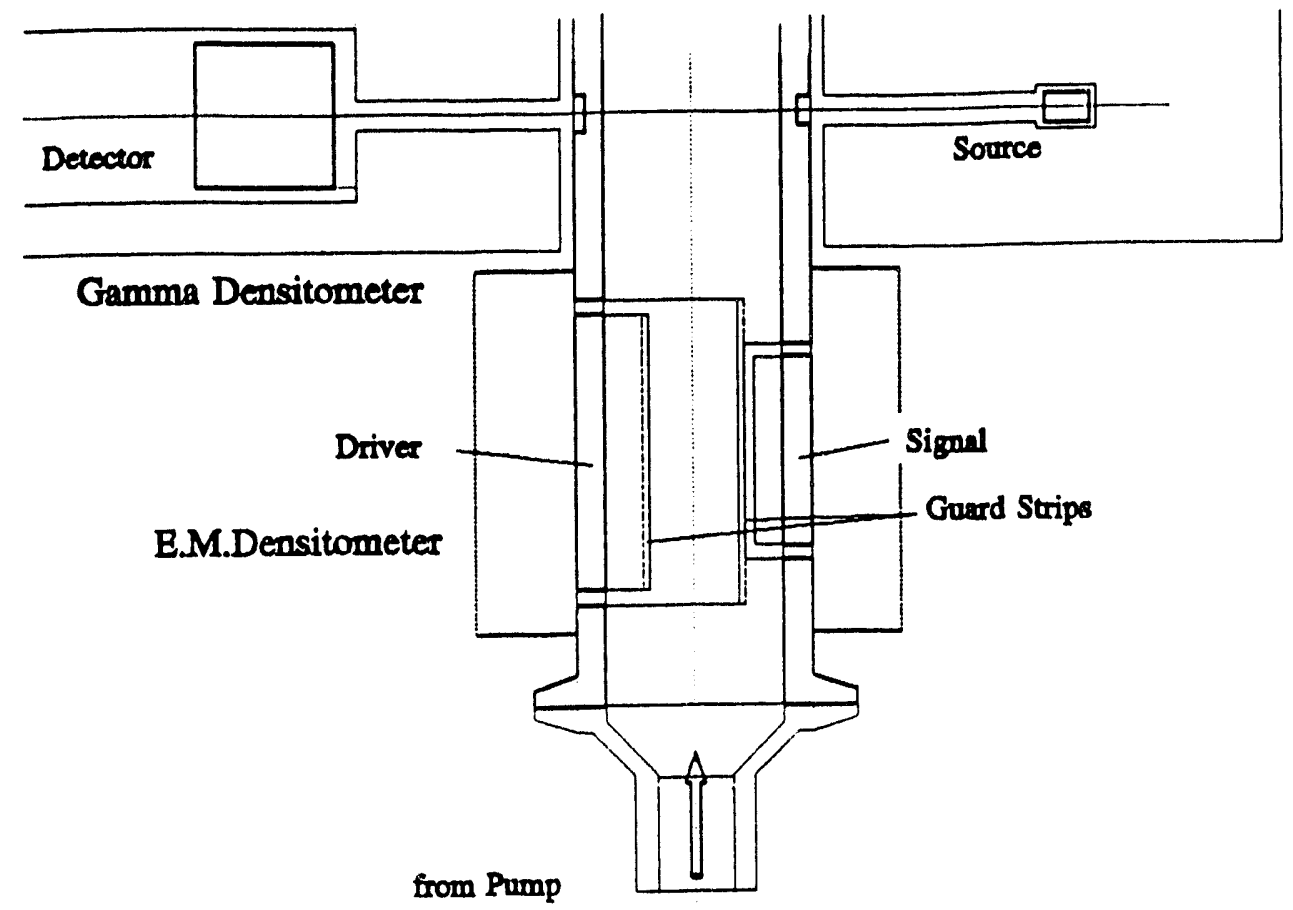

FIG $(V-1)$ : Bench-Top Test Loop for EM Densitometer Calibration

(2) Circular Duct, Self-Calibrating

The attractiveness of self-calibration, i.e., inference of the solid fraction from the readout, a reference readout and calculations that were reviewed in chapter IV, improves with the frequency the solid material is changed and thus requires a new calibration. As methods of measuring the dielectric constant of solids samples are now fairly standard and satisfy the accuracy demanded by self-calibration, the need for this parameter imposes no significant obstacle; the need for extensive on-line computations likewise can be met with relatively inexpensive equipment.

As discussed in Chapter IV, self-calibration requires a sensing geometry that views the duct interior exclusively. That the entire cross section must be sensed with high uniformity when flow may be stratified was also emphasized but is evidently less important for dense slurries, typically showing little stratification and/or Magnus-effect. One might thus conclude that an electrode geometry such as the ANL Mk 2 device's (that avoids sensing outside and covers not all, but a large fraction of the cross section) can produce an essentially error-free reading. Unfortunately, that is not the case: another error, not yet specifically treated, results from the dielectric discontinuity at the insulating wall that separates electrodes (unless field lines run strictly parallel to the wall, as they do only in parallel-plate geometry). This will now be briefly considered. 


\section{(a) Dielectric Discontinuity Error}

We begin this discussion by recalling the elementary fact that the volume sensed by an EM Field device of axial geometry is bounded by electrode surfaces and those field lines that just reach the edge of the signal electrode. Other field lines, terminating on the Guard electrode, pass through the surroundings; some traverse the wall that separates the electrodes and are refracted, to the extent to which the wall surface forms a dielectric discontinuity. The resultant effect on the sensing area is illustrated schematically in FIG $(V-2)$, for ANL $M k 2$ and narrow sensing strip (Auburn) geometry. Sensing area edge Field lines are drawn for three hypothetical dielectric mismatches (the effect is strongly exaggerated for purposes of illustration). Now, since the bulk dielectric constant of the reference fluid differs from that of the slurry, these two measurements will cover a slightly different duct area. Eq. (IV-20), implying identical geometry, must be corrected for this difference, specific for the electrode geometry and dielectric constant of the insulating wall section, as evident from the calculations in ref. (E-2).

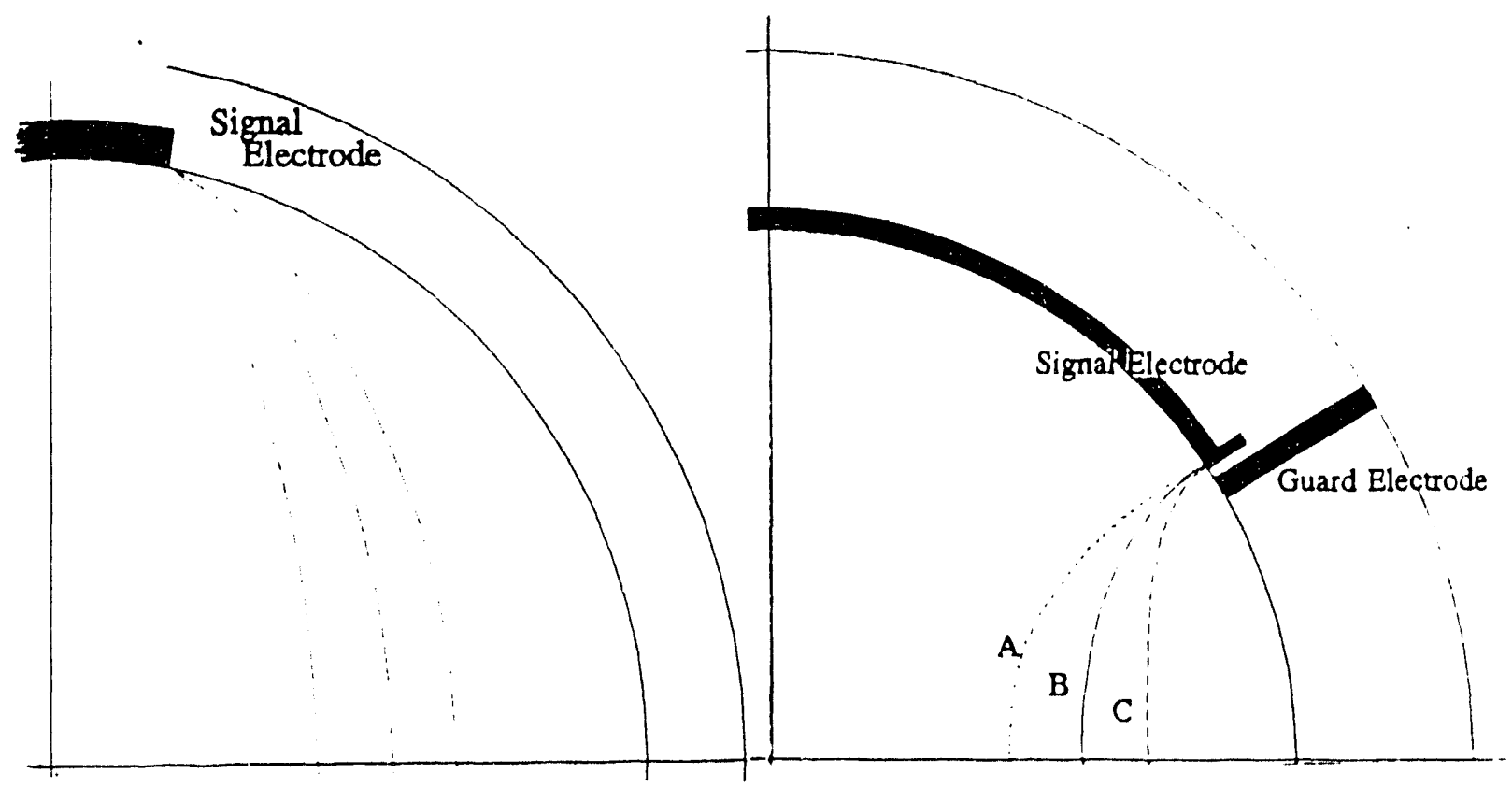

FIG(V-2): Effect of Dielectric Discontinuity, Schematic

Correction appears feasible, yet difficult and involves a process of successive approximation: the solid volume fraction is first calculated with the initial assumption of equal area coverage; then, that value of $\phi$ is used to calculate a new bulk dielectric constant for which the field edges are modeled, yielding a correction for the ratio of capacities hence, a second ap- 
proximation for $\phi$, etc. To execute this routine, a set of field calculations for different bulk dielectric constants must be provided, yielding a Table of covered area vs. bulk dielectric constant that can be stored in the on-line computer memory. The reference measurement likewise needs an accurate calculation of the covered area, supplemented with an accurate measurement that is best made on a Test Loop. All dielectric constants must be corrected for temperature effect, calling for a series of measurements on samples of the solid, or a good measurement at the temperature of the measurement site.

\section{(3) Rectangular (Square) Duct}

The tendency of field lines to bulge towards, or away from, the insulating wall separating signal and driving electrode is absent in Cartesian geometry, allowing a directly comparable reference measurement and providing $100 \%$ duct coverage for any medium. At the same time, the field is highly uniform; all calculations are straightforward. For these persuasive reasons, a square duct section seems preferable to any other configuration for feed line densitometry, where typical duct sizes are not expected to exceed $10-\mathrm{cm}$ diameter.

In any commerci=1 operation, the impedance to flow represented by on-line instruments, reflected in the required pump energy, must be carefully considered. An abrupt transition from circular to square would imply considerable added impedance as well as edge erosion. The transition piece design discussed in the next section deals with this problem: minimizing impedance, it preserves the duct cross sectional area throughout.

\section{(a) Transition Piece Design}

A square, or rectangular, measurement section implies two requirements: first, to provide a smooth transition from circular to square without changing the cross sectional area, secondly, to provide adequate strength or otherwise prevent deformation or rupture of the square duct under high internal pressure and subject to constant erosion. A smooth transition can be effected by fabricating connectors with the profile

$$
\begin{aligned}
x(z) & =3 H(z / L)^{2}[1-2 z / 3 L], \quad 0 \leq z \leq L, \\
\pi R^{2} & =\pi r(z)^{2}+4 r(z) x(z)+x(z)^{2}=H^{2} .
\end{aligned}
$$

where $x(z)$ is the side-length at distance $z$ from the circular end, $R=$ radius of duct, $r=$ radius of corner at $z, L=$ length and $H=$ square side length; $0<x<H, R>r>0$. An isometric view of one quarter of the transition piece is shown FIG (V-3). Fabrication(") may involve casting but is best controlled by machining on a programmable machine tool, in two halves (SS-316 seems a suit-

(*) Prototypes suitable only for limited testing were fabricated by wrapping a mandrel with glass tape soaked in epoxy resin; the result of a few layers was cut lengthwise and removed from the mandrel, then re-glued with epoxy and finished by more wrapping. 
able material for the transition pieces). Square ends are welded or bolted to a Strongback frame that supports electrodes, Guard plates and ceramic blocks between electrodes. Ceramic strips join Electrodes, guard plates and the duct stub, cemented in place with AREMCO, COTRONIC or some other high-strength ceramic-to-metal bonding agent.

\section{(b) Dummy Duct and Pressure Envelope}

To deal with the problem of providing reference pure-fluid measurements at the temperature of the instrument site, a careful measurement, with the duct filled with pure fluid, may be sufficient if corrected for temperature effects (including change in dimensions and change in dielectric properties). However, there are some advantages to a permanent dummy spoolpiece that shares thermal insulation with the on-line capacitor and thus assumes its temperature. The dummy device can be scaled down in size without affecting the readout, and used in a "track-switching" mode to stabilize channel electronics against drift, as discussed further on.

For operation at high pressure, the square section and dummy capacitor may be enclosed in an outer envelope. The space between duct and outer envelope is filled with an inert dielectric liquid; a small expansion chamber, mounted on the duct at some distance from the electrodes, may be provided to equalize pressure on both sides of the duct wall. Re-entrant, thermally insulated and/or circulation-cooled cavities (accessed through end-plates of the outer envelope) should be provided for the electronics. A complete design, suitable for extreme pressures, would evidently entail considerable engineering development as well as cost of fabrication.

\section{(4) Pipeline On-Line Densitometry Design Strategies}

It remains to add some notes on densitometry design strategies for a pipeline. As considered in Chapter III, the solid volume fraction here is sufficiently smaller, and the consist sufficiently coarser, than in a reactor feed line to make the flow mode turbulent and the solids distribution considerably less uniform, possibly somewhat stratified. Calibration, with a representative number of different slurry compositions, is clearly impractical; even a reference measurement with pure water may be difficult to organize.

Pipelines are likely to demand flow measurement at a large number of different sites. A square section could be provided at one such site, and a densitometer could be installed at that point, together with another at an adjacent duct section of circular cross section line. The second densitometer and all other similar densitometers are then calibrated against the error-free square duct unit.

The design of an EM sensing densitometer does not necessarily change with scale; apart from stray capacity, the electronics as well as device geometry are essentially independent of pipe diameter. If the flow mode can be scaled, i.e., the Reynolds' number and consist can be adjusted to a smaller duct size, studies supporting, e.g., the choice of weights for a set of vertical/horizontal plates can be undertaken on a Test Loop. Likewise, the duct area effect by 
fleld line response to the
vestigated in the laboratory.

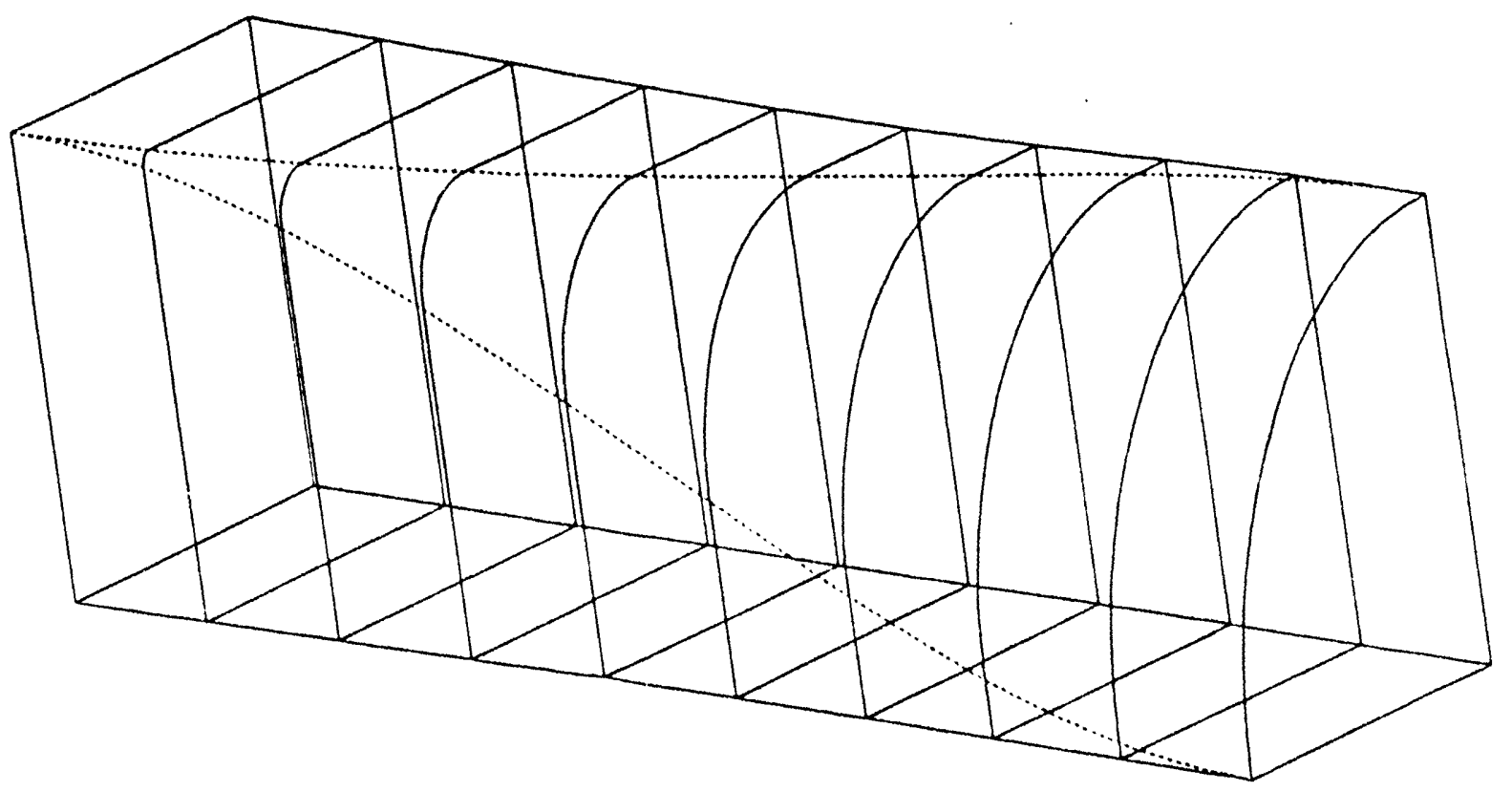

ission system could be developed

For on-site verification, a gamma transmis orientation, allowing a sorts

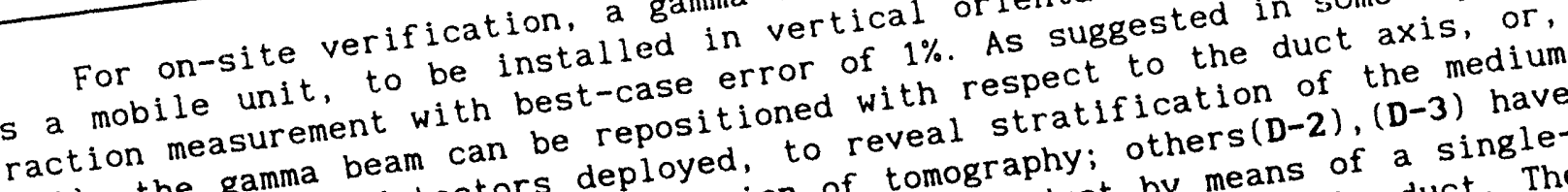
$(D-1)$, the gamma detectors deployedion of tomography; by means of a singleseveral beams and detectementary version of in a duct by meapout the duct. The in rough detail detailed 2-D density rotated eccentrically, however, consider a obtained fairly detal a carriage and useful and interesting, ho to be kept to a beam unit mounted obtainable may be une requires stratification.

information thus flow in a pipeline measures to be necessiod

ing that assured fould not expect such

(B) Gamma Transmission Densitometry

(1) Equipment and Siting Considerations

As indicated in the preceding chapter, gamma transmission can out and a reference readout, well wa 70 
discussed in section (A); we now review some practical aspects.

Collimation is required on both the source and the detector side of the duct, welghing altogether 2-3 tons. Lead, consisting of bricks that may have to be recast or machined to $f i t$, is the princlpal component. Collimator edges and source surround may be fabricated from a machinable tungsten alloy such as "Hevimet". Collimator design can take advantage of some avallable Monte Carlo programs that allow 2-D and 3-D implementation of fairly complex geometries.

The detector should allow the highest possible rate of individual (pulse) counts, eliminating some highly efficient scintillators such as BGO and CsI. Scintillators must deliver a short pulse, yet offer adequate light yield and thus, energy resolution. NaI(Tl) appears as the best compromise choice. If the system is to be used for callbration only, a lower count rate and longer reading intervals can be tolerated, suggesting silicon solid-state detectors a possible alternative to scintillation.

All detectors are temperature-sensitive and must be protected from above $40 \mathrm{deg} C$ or below-freezing temperatures. Ruggedized (Satellite Instrumentation) versions of detector/photomultiplier packages are available.

\section{(2) Electronics: Light Pulser Stabilization}

The discussion in Chapter III emphasized the need for a high rate capability if the error, mainly contributed by counting statistics, is to be reduced to the $1 \%$ level while providing a new reading every few seconds. Operational amplifiers boasting a bandwidth well over $100 \mathrm{MHz}$ have become available in the last few years. The pulse rise-time delivered by these amplifiers is steep enough to allow pulse clipping to 70-100 ns length from the natural $250 \mathrm{~ns} 1 / \mathrm{e}$ length of $\mathrm{NaI}(\mathrm{Tl})$ scintillations. Short pieces of RG-174 or a similar type of miniature coaxial cable, feeding into circuits that match the cable impedance, are a useful and simple means for pulse clipping. Aside from this, the design, and fabrication, of a wideband preamplifier is neither difficult, nor needs further discussion.

Photomultipliers (and, to some extent, scintillation crystals) are subject to aging; gain per stage tends to drift, especially as the count rate varies. To offer reliable service for calibration purposes, the gamma channel electronics must be stabilized, which implies a stable test input in the form of light flashes, controlled by a pulser and produced by a LED. F or best stabilization, the LED spectrum should roughly match the sensitivity curve of the photomultiplier; blue LED types, now avallable, are thus preferred. It is convenient to mount the LED near the PMT base and to guide light pulses to the photocathode by means of optical fibers.

Stabilization schemes for scintillation and proportional counters were formerly configured as Analog Servo-Loops of first order, with the drawback that tracking failed when the count rate declined. Servo-Loops of second order are now readily configured by feeding pulses that fall into adjacent channels to opposite sides of an add-subtract scaler connected in parallel to a digital memory; contents of the latter are translated into a bias voltage applied to the PMT HV unit in paraphase such that, if more pulses fall into the high 
channel, PMT gain is reduced. The bias memory remains constant if input counts cease. Circuits of this type are available or can be constructed with either autonomous or computer-directed control.

\section{(3) Calculations and Measurement of Solids Intrinsic Density}

It was averred in Chapter IV that gamma transmission could deliver an absolute readout of the solid volume fraction if furnished with

(a) the intrinsic densities of solid and fluid,

(b) the Chemical compositions of solid and fluid.

If all principal components are low-Z elements, then instead of (b),

(b') the Hydrogen weight fraction of solid and fluid,

(b") a few of the principal components of solid and fluid

suftice. Some sample calculations, reproduced below, illustrate this. is indicated by these numbers, measurement accuracy is strongly affected by the accuracy of the intrinsic density information, much less affected by the Hydrogen weight fractions of components and only slightly affected by the carbon and iron content of coal. None of the other major or minor constituents of coal $(D-7),(D-8)$ has an effect that exceeds a few tenths of one per cent.

Recalling Eqs. (IV-10) and (IV-11),

$$
\begin{aligned}
& \phi_{s}=\frac{1}{\operatorname{Ag} \times} \log \left(C / C_{f}\right) \\
& A=\Delta \rho[1+a+b], \Delta p=\rho_{s}-\rho_{f} ; \quad([V-11 a) \\
& \mathbf{a}=\mathbf{m}\left[\rho_{\mathrm{s}} \omega_{\mathrm{Hs}}+\rho_{\mathrm{f}} \omega_{\mathrm{Hf}}\right] / \Delta \rho, \quad(I V-11 b) \\
& \mathbf{b}=\left[\rho_{\mathrm{s}} \sum G_{1} \omega_{1 \mathrm{~s}}-\rho_{\mathrm{f}} \sum G_{\mathrm{k}} \omega_{\mathrm{kf}}\right] / \Delta \rho . \quad(I V-11 \mathrm{C}) \\
& \mathrm{m}=2 / \mathrm{A}_{\mathrm{H}}-1=0.984 ; \quad \mathrm{g}=N \sigma_{\mathrm{H}} / 2=.07711
\end{aligned}
$$

Values of the constants $G$ are tabulated for all major and many minor coal constituents in TABLE (IV-1); Fluid density $\rho_{f}$ is readily found and corrected to medium temperature, Hydrogen fraction of the fluid is likewise readily calculated from its Chemical formula. The Hydrogen and Carbon weight fractions of coal are reported in an "Ultimate" Analysis; of all other weight fractions, only the $\mathrm{Fe}$ fraction is needed. This can be inferred from a "Ten Component Ash Analysis" often, through not always, provided for coal that lists ash component element oxides as a fraction of ash weight, together with the "Proximate" Analysis of coal (Ash, Volatiles, Moisture \& Fixed Carbon as a fraction of coal weight). If no "10-Component" Analysis is available, an estimate that is sufficiently accurate in many cases can be calculated from a "Standard Ash" model and a Proximate Analysis. Thus, all the data needed by Eqs. (IV-10) and $(I V-11)$ are available, except solids intrinsic density $\rho_{s}$. 
For a laboratory measurement of that constant, three schemes can be considered. A Helium Pycnometer is widely judged the most reliable means of measuring intrinsic densities(D-6). Pycnometer error-minimizing design is discussed in Appendix C. Liquid Pycnometry with the liquid that is used for slurrying appears as another potentially accurate means. Coal porosity, an important factor in the "intrinsic" density, varies with rank, i.e., with the age of the coal deposit; pores are originally filled with methane (which gradually escapes as coal is mined, crushed, ground and washed). When coal is immersed in a liquid, it swells, presumably as pores become enlarged; heating likewise affects the physical structure of pores while pressure tends to increase fluid penetration.

Pycnometer measurement starts with grinding to $<100$ mesh. Wet measurement. proceeds to mix weighed solids and fluid samples, to the maximum labout 70 solid volume \%). If a measurement at ambient pressure is sufficient, a commercial densitometer (based on sensing the inertial response of a U-tube filled with the samplel is preferable; a polynomial is filted to the measured points and extrapolated to 100\% solids. For higher pressures and temneratures. the procedure and equipment described above, for the Bulk Density measurement, applies.

Recognition that gamma transmission is absolutely unaffected by temperature, pressure or solids distribution along the gamma beam suggests the alternative use of a benchtop Gamma Transmission unit for intrinsic solids density measurement. A vessel is filled to height $h$ with a slurry made of the solid at specified $\phi$ with any convenient fluid and inserted into the vertical gamma beam; the measurement is repeated with pure fluid. The foregoing equations are then applied to the measurement to extract $\rho_{i s}$ :

$$
\rho_{s}=\left[\frac{1}{\operatorname{gh} \phi_{s}} \log \left(C / C_{f}\right)+\rho_{t}\left(1+m \omega_{H t}+\left[G_{k} \omega_{k f}\right)\right] /\left(1+m \omega_{H s} \cdot \sum_{i} \omega_{i s}\right)\right.
$$

A single measurement suffices, however, a series with different values of $\phi_{\mathrm{s}}$ can be occasionally run for verification. The bench-top unit does not need special wideband electonics but must provide Good-Geometry collimation.

Table (V-1) lists factors $G_{1}$ and weight fractions $\omega_{1 s}$ for major constituents of Ohio \# 9 coal, a high-ash bituminous type suitable for Gasification of intrinsic density $=1.51$. Weight fractions are in part from an Ultimate \& Proximate Analysis (C, H, N, S, Ash \& Moisture) and in part from a 10-Component Ash Analysis(D-9) in terms of Oxides, listed in Table (V-2) together with translation to Metal weight fractions of ash, then weight fraction of Coal (using the "Ash Fraction" of the Proximale Analysis). These are used in Table $(V-1)$ except for the Oxygen value, estimated from Ultimate and Sulfur Analyses. As readily apparent, products $G_{1} \omega_{1 s}$ are negligibly small except for $\mathrm{C}, \mathrm{Al}$ and Fe; the Sum of products amounts to less than $0.5 \%$. For the fluid we list water and Diala-AX oil, a benign Transformer oil (Shell) with specific gravity $\rho_{\mathrm{f}}=0.886$ and $86.5 \% \mathrm{C}$ by weight, corresponding to a formula $\mathrm{CH}_{1.86}$. 
TABLE $(V-1):$ COMPUTATION FACTORS FOR MAJOR CONSTITUENTS OF OHIO \# 9 COAL AND TWO FLUIDS

\begin{tabular}{|c|c|c|c|c|c|c|c|c|}
\hline Elemt & 2 & $\omega_{s Z}$ & $\mathrm{G}_{\mathbf{z}}$ & $\omega_{s} G_{z}$ & $\omega_{f Z}^{\prime}$ & $\omega_{f z}^{\prime} G_{z}$ & $\omega_{\mathrm{rz}}$ & $\omega_{r z} G_{z}$ \\
\hline $\mathrm{H}$ & 1 & .0435 & - & - & .135 & & 119 & \\
\hline C & 6 & .5961 & -.001394 & -.00106 & .865 & -.001384 & - & \\
\hline 0 & 8 & .0514 & +.001777 & +.000914 & - & - & .888 & +.00158 \\
\hline $\mathrm{N}$ & 7 & .0086 & -.000251 & -.000002 & & & & \\
\hline Al & 13 & .0324 & -.031719 & -.00103 & & & & \\
\hline S & 16 & .0436 & +.005811 & +.00025 & & & & \\
\hline $\mathrm{Si}$ & 14 & .0634 & +.003753 & +.00024 & & & & \\
\hline $\mathrm{Na}$ & 11 & .0006 & -.039312 & -.000024 & & & & \\
\hline K & 19 & .0047 & -.016688 & -.000078 & & & & \\
\hline $\mathrm{Ca}$ & 20 & .0032 & +.009723 & -.000031 & & & & \\
\hline $\mathrm{Fe}$ & 26 & .0289 & -.047475 & -.003720 & & & & \\
\hline $\mathrm{Ti}$ & 22 & .0013 & -.067780 & -.000088 & & & & \\
\hline
\end{tabular}

TABLE $(V-2):$ 10-COMPONENT ASH ANALYSIS, OHIO \# 9

\begin{tabular}{|l|c|c|c|}
\hline Species & Oxide wt $\%$ & Metal wt $\%$ & $\%$ of Coal \\
\hline $\mathrm{SiO}_{2}$ & 53.9 & 25.8 & 6.344 \\
$\mathrm{Al}_{2} \mathrm{O}_{3}$ & 24.9 & 13.18 & 3.241 \\
$\mathrm{Fe}_{2} \mathrm{O}_{3}$ & 16.8 & 11.75 & 2.89 \\
$\mathrm{TiO}_{2}$ & 0.9 & 0.54 & 0.13 \\
$\mathrm{P}_{2} \mathrm{O}_{5}$ & 0.11 & - & \\
$\mathrm{CaO}$ & 1.8 & 1.3 & .32 \\
$\mathrm{MgO}$ & 0.7 & 0.42 & 0.10 \\
$\mathrm{Na}_{2} \mathrm{O}$ & 0.3 & 0.22 & 0.055 \\
$\mathrm{~K}_{2} \mathrm{O}$ & 2.3 & 1.91 & 0.47 \\
$\mathrm{SO}_{3}$ & 0.3 & - & \\
\hline
\end{tabular}

The terms of Eq (IV-10), calculated for Ohio \# 9 coal, are tabulated in TABLE $(V-3)$. Note that, even though this is a High-Ash coal with especially large corrections for $\mathrm{Fe}$ and $\mathrm{Al}$, the $\mathrm{b}$ term is still slightly less than $1 \%$.

TABLE (V-3): TERMS OF EQ. (IV-1C) FOR OHIO \# 9 COAL

\begin{tabular}{|l|l|c|c|}
\hline \multicolumn{1}{|c|}{ TERM } & \multicolumn{1}{|c|}{ FORMULA } & (0i l) & (Water) \\
\hline Density Contrast & $\Delta \rho=\rho_{\mathbf{s}}-\rho_{f}$ & .624 & .500 \\
Hydrogen Correction & $\mathbf{a}=\rho_{\mathrm{s}} \omega_{\mathrm{sH}}-\rho_{\mathrm{f}} \omega_{\mathrm{fH}}$ & -.08646 & -.08921 \\
Final Correction & $\mathbf{b}=\left[\rho_{\mathrm{s}} \sum \omega_{\mathrm{sj}} \mathrm{G}_{\mathrm{j}}-\rho_{\mathrm{f}} \sum \omega_{\mathrm{fk}} \mathrm{G}_{\mathrm{k}}\right] / \Delta \rho$ & -.009287 & -.0168 \\
\hline
\end{tabular}


Results of or-line Ohio \# 9 coal-water slurry measurements at the ANL-SLTF are shown in TABLE $(V-4)$. The difference ratio $\Delta \phi_{\mathrm{s}} / \phi_{\mathrm{s}}$ between grab-sample and gamma transmission measurements is evidently insignificant, allowing for some uncertainty in the pycnometric work as well as in the transmission measurements (that did not use stabilized electronics).

TABLE (V-4): EXPERIMENTAL

\begin{tabular}{|c|c|c|}
\hline$\phi($ pyc $)$ & $\phi(\gamma \mathrm{Xm})$ & $\Delta \phi / \phi(\mathrm{pyc})$ \\
\hline .191 & .192 & -.0052 \\
.280 & .279 & +.0036 \\
.311 & .318 & -.0258 \\
.440 & .443 & -.0068 \\
.551 & .558 & -.0127 \\
\hline
\end{tabular}

For completeness, calculated corrections for a low-ash coal (Inland mine, IL, Survey Sample \#C-15868, $\rho_{S}=1.38$ ) are presented below, in $\operatorname{TABLE}(\mathrm{V}-5)$ :

TABLE $(V-5)$ : COMPUTATION FACTORS FOR MAJOR CONSTITUENTS OF C-15868 (INLAND) LOW-ASH COAL AND TOLUENE

\begin{tabular}{|l|c|c|c|c|c|c|}
\hline Elemt & $Z$ & $\omega_{\mathrm{s} Z}$ & $\mathrm{G}_{\mathrm{Z}}$ & $\omega_{\mathrm{s} Z} \mathrm{G}_{\mathrm{Z}}$ & $\omega_{\mathrm{f} Z}^{\prime}$ & $\omega_{\mathrm{f} Z}^{\prime} \mathrm{G}_{\mathrm{Z}}$ \\
\hline $\mathrm{H}$ & 1 & .0490 & - & - & .0875 & \\
$\mathrm{C}$ & 6 & .7513 & -.001394 & -.001047 & .9118 & -.001271 \\
$\mathrm{O}$ & 8 & .0877 & +.001777 & +.000155 & - & - \\
$\mathrm{N}$ & 7 & .0152 & -.000251 & -.000004 & & \\
$\mathrm{Al}$ & 13 & .0130 & -.031719 & -.000412 & & \\
$\mathrm{~S}$ & 16 & .0085 & +.005811 & +.000049 & & \\
$\mathrm{Si}$ & 14 & .0238 & +.003753 & +.000089 & & \\
$\mathrm{Na}$ & 11 & .0010 & -.039312 & -.000034 & & \\
$\mathrm{~K}$ & 19 & .0017 & -.016688 & -.000078 & & \\
$\mathrm{Ca}$ & 20 & .0091 & +.009723 & -.000088 & & \\
$\mathrm{Fe}$ & 26 & .0048 & -.047475 & -.000223 & & \\
$\mathrm{Ti}$ & 22 & .0006 & -.067780 & -.000041 & & \\
\hline
\end{tabular}

Toluene, used in HIGAS, is adopted here as the silurrying fluid; one finds that

$$
\begin{array}{lll}
\rho_{\mathrm{s}} \omega_{\mathrm{Hs}}=.06762 ; & \rho_{\mathrm{f}} \omega_{\mathrm{HF}}=.08753 ; & \mathbf{a}=-.03844 \\
\sum \omega_{\mathrm{Js}} G_{J}=-.001634 ; & \sum_{\mathrm{kf}} \omega_{\mathrm{k}}=-.001271 \text { (Toluene); } & \mathbf{b}=-.000701
\end{array}
$$

whence $\mathbf{b}$ is negligible and even a is quite small for this example. One would expect most types of coal to fall within these limits, noting especially that the gamma transmission scheme is quite insensitive to sulfur content and that, on the other hand, a hydrogen correction is significant for all slurries, particularly for those based on water. 


\section{(C) EM Sensing Densitometry}

\section{(1) Hardware Design Notes}

EM sensing electrode configurations for circular and for square duct profiles have been developed in Chapter III and further discussed in Section (A) of this Chapter. It remains only to add a few details, shown in the sketches reproduced in FIG $(V-4)$ and $(V-5)$. FIG $(V-4)$ shows a possible design for a 2-in Sch. 40 duct. Electrodes are sliced from SS-316 pipe; 0.125 -in thick plates are welded to electrode edges as indicated. SS-316 sheet-stock Guard electrodes and Signal electrode are bonded through intermediary ceramic fillets $B$ that can be fabricated from machinable ceramic. The Driver electrode is fabricated to the same dimensions, except that the intermediate ceramic fillet is replaced by steel and axial length extends 1.5 in beyond the signal plates. Guard strips extend 2 -in beyond signal plates in the axial direction and are welded at both ends to 4-in long pipe stubs that are equipped with standard flanged connectors (preferably of a type that can be accurately aligned).

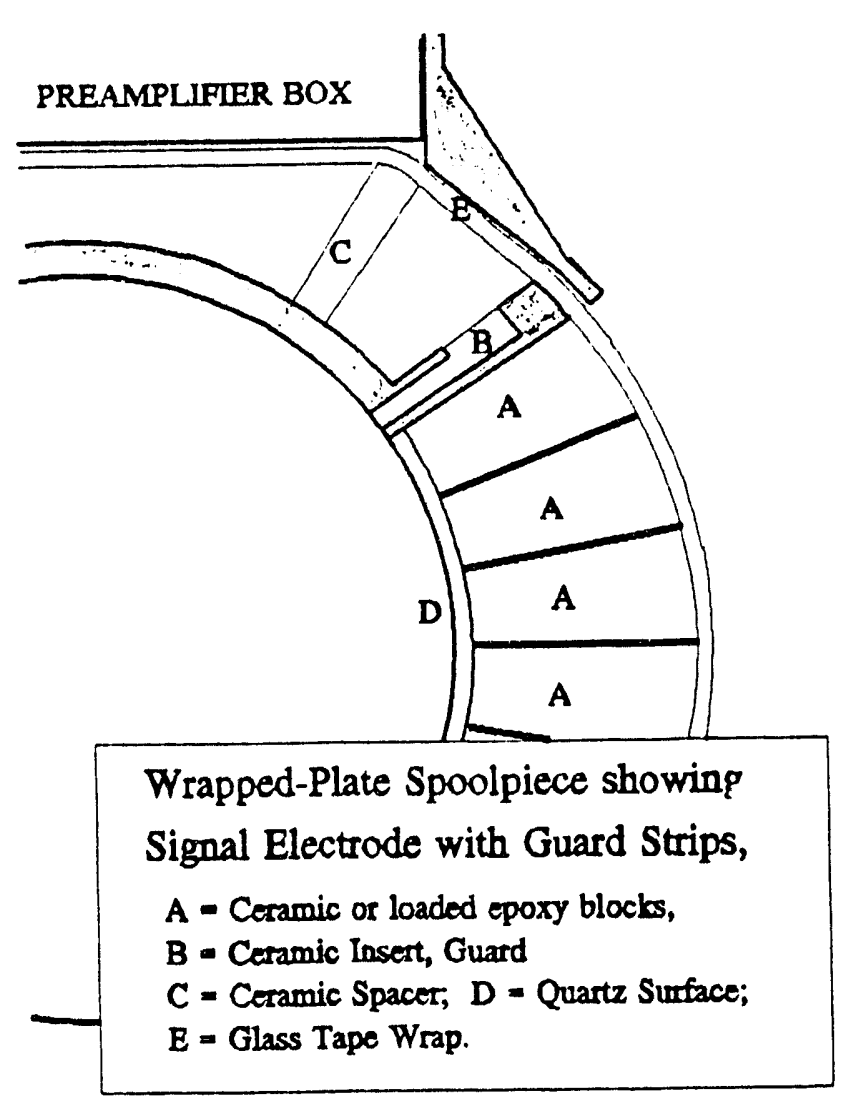

FIG(V-4): Design Details, ANL Mk 2 EM Sensing Spoolpiece, Circ. Duct
To form the two lateral insulating sectors, dielectric blocks A, 0.75 -in wide, are interleaved with radial nickel shim-plates. This assembly likewise extends slightly beyond the Driver Electrodes at both ends; foils protrude beyond the ceramic block structure at one end to attach voltage divider network components. The blocks can be fabricated from ceramic, or cast with barium-titanate-loaded epoxy resin to produce some desired dielectric constant (cf. preceding discussion on dielectric mismatch error). The inner surface of the insulating sectors is covered with a curved piece D of fused quartz or hardened glass, sliced from tubing.

Leads are welded to the top of the signal and driver electrodes; the entire unit is assembled with bonding agent; spaces outside duct walls are filled with ceramic-loaded resin, with ceramic standoff slabs $C$ emplaced as shown. The duct is wrapped with multiple layers $\boldsymbol{E}$ of glass tape soaked in resin. Holes are drilled and tapped to attach brackets to the Guard plates.

Brackets secure Preamplifier and Oscillator boxes to the unit. Cable connections enter the circuit boxes at either end. 
To promote structural integrity, relieve strain on ceramic seals and serve as an external electrical shield, split plates, 4.5 in by 4.5 in, are clamped to the pipe stubs at both ends and interconnected with a box, assembled from aluminum plates. For higher duct pressure and temperature, a wore complex design, including thermal insulation layers of such materials as FIBERFRAX, is needed; thermal expansion coefficients of materials must be matched and parts thickened. An outer envelope consisting of a 4-in Sch 80 pipe with heavy end-walls welded to the end-stubs, is filled with a dielectric fluid, e.g., 3-M FLUOROINERT. A Tee on one of the pipe stubs supports a diaphragm or one-turn bellows for pressure equalization.

FIG(IV-5) is a sketch of an assembled prototype square duct that can be disassembled for inspection and cleaning. Signal, Guard and Driver electrodes

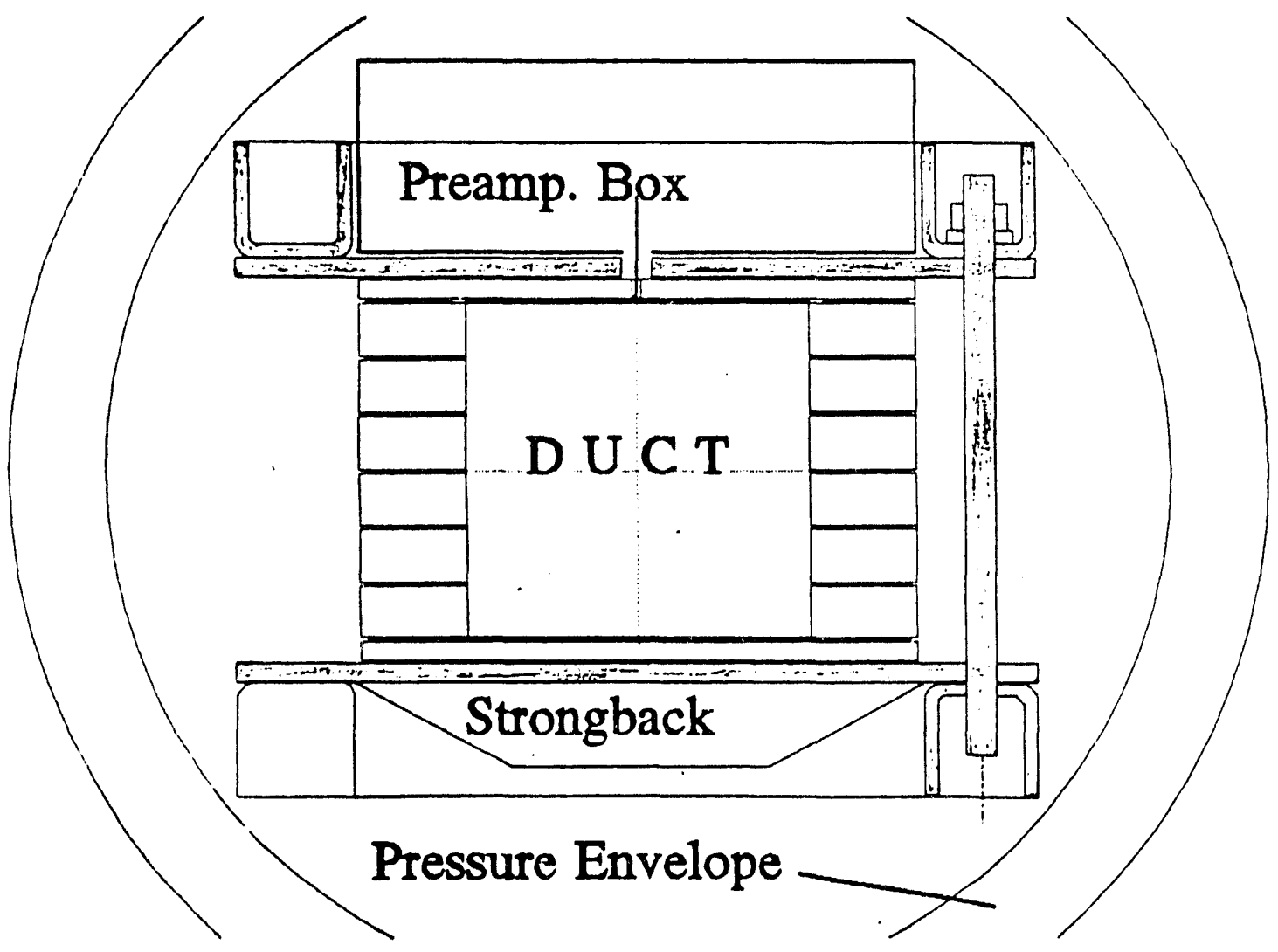

FIG( V-5):Design Details, EM Sensing Spoolpiece for Square Duct

of 0.065 -in SS-316 sheet stock are bonded to 0.25 -in ceramic slabs drilled to allow clearance for leads welded to signal and driver plates. 0.25-in thick steel plates welded to channels are held against the ceramic slabs by a number of lateral bolts. Transverse Strongback ribs welded to top and bottom external plates resist deformation. The insulating sides of the box consist of ceramic slabs and nickel foil, again protruding at one end to allow connection to the Voltage Divider; blocks and foils are bonded with ceramic cement.

Forming a seal with 0.003-in thick Teflon sheet between side-walls and driver plate, the duct is assembled by tightening bolts with a torque wrench. 
At the same time, the strongbacks are clamped to the square ends of transition pieces (whose round ends are flanged to allow insertion into a circular duct). Circuit boxes can be fitted as indicated, into the compartments formed by the rails and transverse sheet-stock reinforcements of the Strongbacks. The entire unit can be encased in an external circular 6-in pipe or aluminum sheet box.

\section{(2) Electronics: Channel Switching Stabilization}

The full benefit of a design that can dispense with calibration can only be realized by a unit whose electronic component is stable enough to dispense also with frequent checkout or re-zeroing. Analog amplifiers are not stable enough to deliver a readout that remains within $1 \%$ over months of continuous operation; feedback stabilization is always useful but, in the long run, cannot cope with component aging effects.

The circuitry involved in EM Field sensing involves, at the driving side, an oscillator turning out a high-frequency signal of amplitude $E$ and frequency f. Within broad limits, the frequency does not affect the readout, hence, frequency stability is not an issue. However, the preamplifier readout is directly proportional to $E$, whose possible instability must be compensated for.

The preamplifier itself can be stabilized by internal feedback. After the preamplifier, a diode bridge rectifier converts to DC; high-frequency components of the signal are trimmed by a low-pass filter designed for good phase stability and reasonably sharp cutoff characteristics, such as a Bessel filter. The diode bridge output varies with temperature and is thus subject to drifts that cannot be eliminated by feedback; likewise, the following DC amplifier tends to have threshold and gain drifts. Its output is sampled by an ADC circuit or digitized in a VFC, the latter offering immunity to aliasing but somewhat poor stability at the wide frequency range $(\approx 2 \mathrm{MHz})$ demanded by the "frequent sampling" scheme discussed in the foregoing.

Certain difficult measurements in Observational Astronomy (e.g., of the radiation intensity of single stars or galaxies) have long made use of "trackswitching", an arrangement in which a reference signal and the signal to be measured are alternately passed through the same electronic unit: gain fluctuations are canceled in the unknown to reference output ratio. As noted repeatedly in the preceding, EM sensing requires such a ratio, $C_{m} / C_{f} . C_{f}$, the capacity measured with the duct filled with pure fluid, can be made available at any time by providing an adjustable dummy capacity designed to match the temperature coefficient of the liquid dielectric. One careful calibration run with pure fluid suffices to trim the dummy to exactly equal the capacity of the on-line device. The dummy and device are connected as indicated in FIG $(I V-5)$, via DPDT mercury-wetted relay to the inputs of iwin preamplifiers connected to twin channels, right up to the computer (digital) input. A low switching frequency is sufficient to make thermal drift and aging effects in each chain negligible from one set of readings to the next.

Let overall Channel Gains $G_{A}, G_{B}$ be initially equalized, then, at some later time, Chain $A$ gain drift by $\delta G_{A}$ and chain $B$ by $\delta G_{B}$, where both magnitude and sign of $\delta G_{A}, \delta G_{B}$ are assumed to be entirely uncorrelated.A reading with $A$ 
connected to $C_{m}$ ard $B$ to $C_{f}$ yields the pair of digital numbers

$$
N_{m}(1)=C_{m}\left[G+\delta G_{A}\right], \quad N_{f}(1)=C_{f}\left[G+\delta G_{B}^{*}\right] ;
$$

Successive switch reversals yield

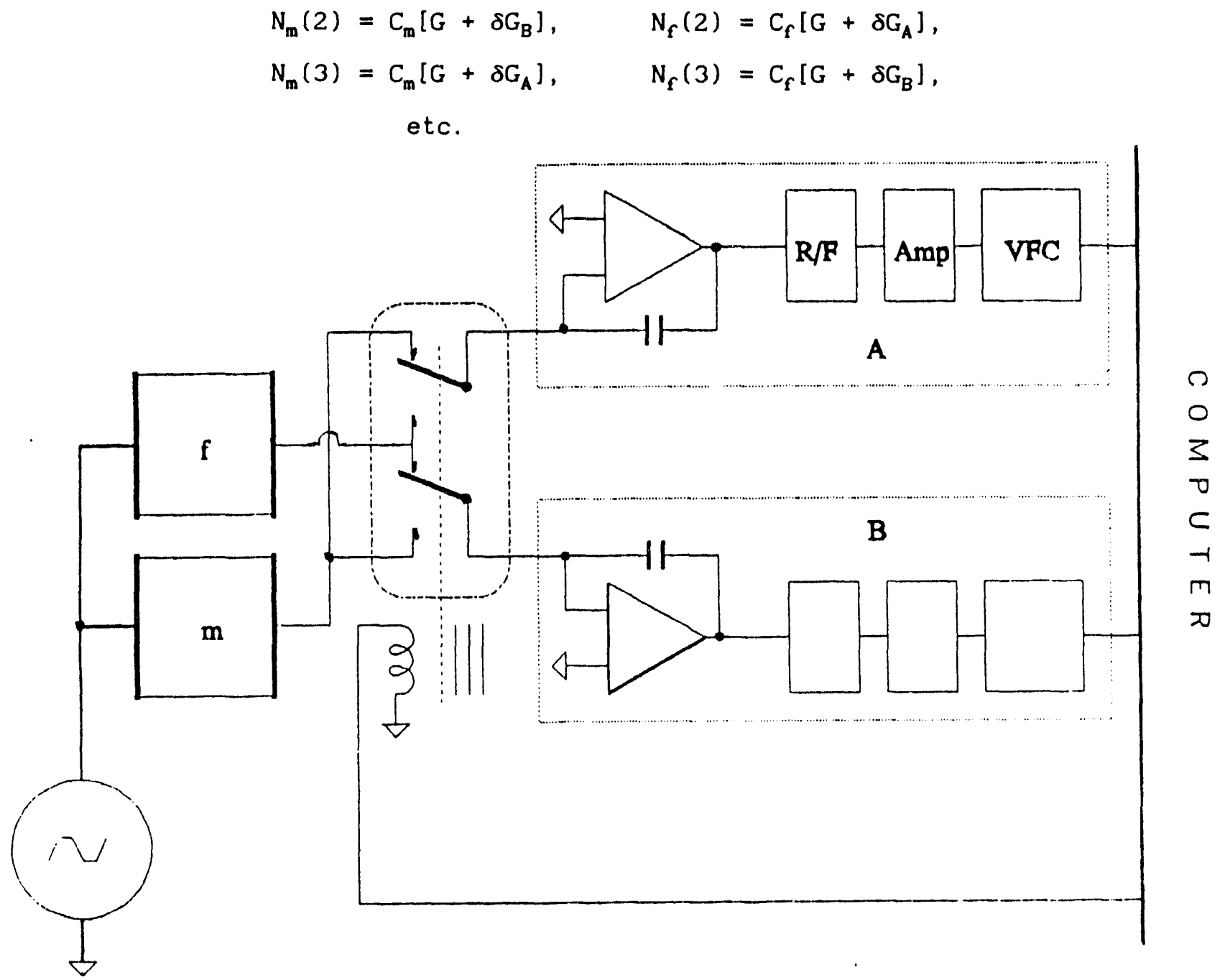

FIG(IV-6): Track-Switching Channels, schematic

If the switching frequency just equals the readout frequency, exactly one pair of samples is delivered for each configuration change, as indicated. In I ractice, sampling frequency may be much higher, whence each of the configurations actually consists of many individual samples. For individual samples or sets, drifts are cancelled by pairing duct readings with dummy readings from the preceding configuration:

$$
N_{m}(i) / N_{f}(i-1)
$$

Unity is subtracted from these digitally computed ratios to obtain the driftfree parameter 


$$
N_{m} / N_{f}-1=\left(C_{m}-C_{f}\right) / C_{f}=3 g \phi_{s}\left(1+g \phi_{s}+g^{2} \phi_{s}^{2} / 3\right),
$$

from which (assuming the duct is rectangular) $\dot{\phi}_{\mathbf{s}}$ can be directly calculated. The overall arrangement is shown schematically in FIG (V-6).

\section{VELOCITY MEASUREMENT}

\section{(A) Introduction}

Specifications for solids feed rate measurement in industrial slurry conveyors, reviewed in chapter $I$, include the requirement of non-intrusive means and the more difficult need for an absolute readout; the first mandated by the abrasive nature of the medium and the danger of plugging, the second by the cost and difficulty of calibrations (that must be repeated every time the medium varies). Subsequent chapters dealt with the on-line measurement of density in the framework of these requirements. We now turn to the other component of a complete instrument scheme: measurement of the duct-average velocity, volumetric flow rate, or mass flow rate.

For uniform, homogeneous media, any flow velocity measurement device constitutes a compiete Flow meter, usually referred to as such. A list of commercially available flow meters was reviewed in Chapter $I$ and winnowed for non-intrusive types, leaving a shorter list from which certain types were eliminated on the basis of inherent error, hence, inability to dispense with calibration; others, potentially accurate, were found to have other inherent limitations. This classification is reexamined in the following section. To put flow velocity measurement into perspective, two absolute-reading, highly accurate, but impractical velocity measurement schemes are added to the list of those that are commercially available and commented on; the former are once more listed in TABLE (VI-1), followed by detailed reviews of each listed type, while TABLE (VI-2) lists the two absolute-reading schemes, again followed by comments.

Table (VI-2) also lists an on-line flow velocity scheme, Cross-Correlation (CCF) Flow measurement, that has some evident similarities with the other two schemes in that Table but differs from them in requiring only simple, readily provided sensing means. To implement CCF of $f$-line calls for a substantial memory that can pick up a 3-4 digit number in a few milliseconds; on-line implementation requires the execution of a multiply-add operation in less than 10 $\mu s$. Neither requirement could be met until recent progress in computer technology eliminated this obstacle to the application of CCF in flow measurement. A remaining problem that needs to be addressed is that, al though the CCF scheme produces a direct velocity readout in principle, this readout must be corrected, in practice, by a "meter factor" that capriciously varies between less and more than unity and between a few to over 50 per cent. As the meter factor requires calibration and even with that, the method has so far worked only for a limited velocity range and strongly turbulent flow, little use has been made of it in industrial plants. This problem is taken up in the following, after a review of other means of flow measurement. 


\section{(B) Review of Available Techniques}

Maximum duct sizes in Table (VI-1) refer to of $f$-the shelf devices; accuracies are as claimed by vendors. Some of the schemes can be customized for larger ducts; costlier versions with better accuracy are available for others.

TABLE (VI-1): COMMERCIALLY AVAILABLE, NON-INTRUSIVE FLOW METERS

\begin{tabular}{|l|c|c|c|}
\hline \multicolumn{1}{|c|}{ Type of device } & max. duct size & readout & accuracy \\
\hline SS-Diagonal Path & 12 in & transit time & $1 \%$ \\
SS-Doppler & $3-4$ in & freq. (shift) & $1-2 \%$ \\
Magmeter & $3-4$ in & velocity & $0.2-1 \%$ \\
Coriolis' Force FM & 1.5 in & mass flow & $1 \%$ \\
\hline
\end{tabular}

\section{(1) Acoustic Diagonal-Path and Acoustic-Doppler}

The first two schemes interrogate the medium by means of compression (acoustic or Supersonic, hereinafter referred to as SS) waves (V-1). Both are commercially available from many vendors, using either clamp-on or built-in transducers. As the schemes share the advantages, as well as some of the generic problems, of all Ss-type sensors, it is instructive to discuss thern in some detail.

The Diagonal-Path (DP) Scheme, much used in homogeneous fluid and/or dilute slurry flow rate measurement, sets a pair of acoustic transducers such that the direct path $S=D / \sin \Theta$ between transducers runs diagonally across a duct of diameter $D$, at the angle $\Theta$ to the axis.

Let

$$
\begin{aligned}
\Delta S_{1} & =i \text { th of } N \text { path segments; } \\
L & =\sum \Delta S_{1} \cos \vartheta_{1}, \vartheta_{1}=\text { angle between axis and local path } \Delta S_{i} ; \\
D & =\sum \Delta S_{k} \sin \vartheta_{k}=S \text { sin } \Theta ; \\
V_{1} & =\text { local stream velocity }(0.001-0.004 \text { of the sound velocity } U \text { ), } \\
\Delta t_{d} & =\text { sonic pulse segment transit time in the downstream direction, } \\
\Delta t_{u} & =\text { sonic pulse transit time in the upstream direction, } \\
t_{u}, & t_{d}=\text { overall (wall-to-wall) transit times, }
\end{aligned}
$$

$\theta_{u}, \theta_{d}=$ angles, indicated in FIG $(V-1)$, between the axis and the path relative to the moving medium, in the up- and downstream direction, respectively.

Choosing Segment lengths $\Delta \mathrm{S}_{i}$ so as to make Segment transit times $\Delta t_{u}$ and $\Delta t_{d}$ as well as angles $\theta_{u}, \theta_{d}$ constant, $t_{u}=N \Delta t_{u}, t_{d}=N \Delta t_{d}$.

In the familiar river ferry analogy, sound pulses moving diagonally downstream "steer" towards point A in Fig ( $V-:)$; upstream pulses towards point $B$, whence 


$$
\begin{aligned}
& \Delta S_{1} \cos \vartheta_{1}=\Delta t_{u}\left[U \cos \theta_{u}-V_{1}\right]=\Delta t_{d}\left[U \cos \theta_{d}+V_{1}\right], \\
& \Delta S_{1} \sin \vartheta_{1}=\Delta t_{d} U \sin \theta_{d}=\Delta t_{u} U \sin \theta_{u}
\end{aligned}
$$

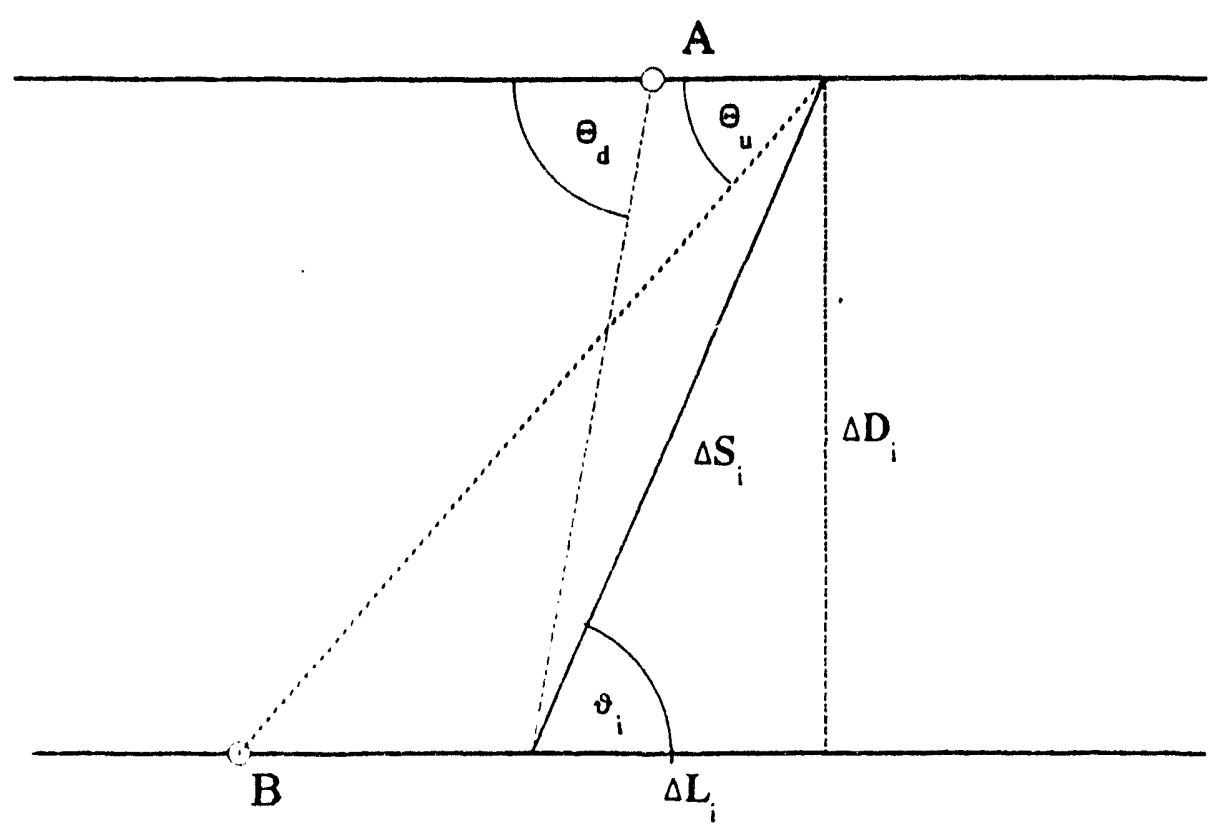

FIG (VI-1): Acoustic Diagonal Path Scheme, schematic.

Summing over $N$ segments and putting $\sum V_{1} / N=V^{*}$,

$$
\begin{gathered}
L=U t_{u} \cos \theta_{u}-V^{*} t_{u}=U t_{d} \cos \theta_{d}+V^{*} t_{d} ; \quad D=U t_{u} \sin \theta_{u}=U t_{d} \sin \theta_{d} ; \\
S^{2}=L^{2}+D^{2}=\left(U t_{u}\right)^{2}\left[1-\left(V^{*} / U\right)^{2}\right]-2 L V^{*} t_{u}=\left(U t_{d}\right)^{2}\left[1-\left(V^{*} / U\right)^{2}\right]+2 L V^{*} t_{d}
\end{gathered}
$$

whence, rearranging and dropping $\left(\mathrm{V}^{*} / \mathrm{U}\right)^{2}<0.001$,

$$
V^{*}=\left(U^{2} / 2 L\right)\left[t_{d}-t_{u}\right] \text {, }
$$

scaling the measured Pulse transit time difference to the "averaged" velocity $V^{*}$. Since $U$ varies with medium temperature, Eq. (VI-1) requires a calibration at the temperature of the measurement site(*).

Straightforward though the scheme may appear, it should be now be pointed out that the velocity $V^{*}$ computed by $E q(V I-1)$ is not the true duct-average, but a quasi-linear average: for example, the true average for a duct of circular cross section comes to

(*) Note however that in a test loop, the sound velocity in any medium at any temperature can be directly measured on-line, by stopping the flow. 


$$
\bar{V}=\frac{2}{R^{2}} \sum V_{1} r_{1} \Delta r=\frac{8}{D^{2}} \sum_{1=1}^{N / 2} i V_{1}\left[U t_{u} \sin \theta_{u} / N\right]^{2}=\frac{1}{N} \sum \frac{1}{N} V_{1}
$$

where we have put $\Delta r=\Delta S_{1} \cos \vartheta_{1}=U t_{u} \sin \theta_{u} / N$ and $r_{1}=1 \Delta r$; in the same terms,

$$
V^{*}=\sum_{l=1}^{N / 2} v_{1} \Delta r / R=\left[V_{1}\left[U t_{u} \sin \theta_{u} / N R\right]\right.
$$

Passing from sum to integral and representing the velocity distribution for turbulent flow by the Prandtl expression,

$$
V=V_{c}\left(1-\frac{r}{R}\right)^{\frac{1}{n}}
$$

one finds that $V^{*}=V_{c} n /(n+1), \quad \bar{v}=2 V_{c} n^{2} /(n+1)(2 n+1)$, hence,

$$
V^{*}=(1+1 / 2 n) \bar{V} \text {. }
$$

As discussed in Chapter III, turbulent flow of a low-viscosity fluid such as water is best modeled by $\mathrm{n}$ ranging from 7 to 10; lower indices, from 3 to 6, characterize the turbulent, or "transition", flow of a slurry. This leads to an error estimate between $7 \%(n=7)$ and $11 \% \quad(n=4)$ for the diagonal path read-out scheme and turbulent flow of a slurry. The instrument makes an error of at least $5 \%$ for pure fluid flow, however that is considered a "meter factor" since calibration is normally assumed. Supposing that the instrument is calibrated for $n=7$, then the error of the calibrated readout is only $-1.5 \%$ for $n=9$ and $+1.0 \%$ for $n=6$, encompassing a very wide range of flow velocities for a uniform fluid. For laminar-Newtonian or Pseudoplastic flow, characteristic of dense slurries, the calibration scheme fails; moreover, dense slurries tend to scatter most of the acoustic energy, hence sonic (compression) waves that reach the receiving transducer at all spread over many paths (whose average cannot be predicted by available theory). It thus becomes clear that the SSDiagonal Path scheme, highly suitable for dilute slurries that allow calibration against another instrument or diverter, is less suitable for dense slurries, and entirely unsuitable for dense slurries flowing in laminar mnde.

The second SS scheme, also commercially available $(V-2)$, makes use of the Doppler wavelength shift of sonic waves that are scattered by moving particles. It needs just a single sonic transducer that can be mounted externally on the duct surface $(\mathbf{V}-3)$. In notable contrast to the preceding scheme that integrates over a path traversing the duct, the Doppler scheme senses individual particles. I argument similar to the foregoing derives the Doppler relation between the transducer wavelength $\lambda$, wavelength $\lambda_{i}$ of the back-scattered wave, particle velocity $V_{1}$ and sound velocity $U$ : for a transducer beam pointing downstream at angle $\vartheta$, one finds that 


$$
\lambda^{\prime} v=U-v_{1} \cos \vartheta ; \quad \lambda \nu=U
$$

- hence

$$
\begin{gathered}
\lambda_{i}^{\prime}=\lambda\left(1-v_{1} \cos \vartheta / U\right), \\
v_{1}=(U / \cos \vartheta)\left(\lambda-\lambda_{1}^{\prime}\right) / \lambda
\end{gathered}
$$

The observed aggregate shift is the result of scattering by many individual particles traveling at different -locities, along the path covered by the sonic beam. The resulting wavelength distribution resembles the velocity distribution, to the extent to which the sonic beam path yields a fair sampling of the duct cross section (in contrast, the diagonal path scheme just measures the wall-to-wall transit time of sonic pulses).

The "sampling efficiency" of a parallel beam of diameter $2 a$ comes to

$$
\begin{aligned}
& E(r)=(2 / \pi) \arcsin (a / r), \quad a<r<R, \\
& \text { (VI-5a) } \\
& E(r)=1 \text {, } \\
& 0<r<a \text {. } \\
& \text { (VI-5b) }
\end{aligned}
$$

Since $\mathscr{E}(r)$ declines steeply as $r$ approaches the duct radius $R$ and the medium velocity declines, the measured wavelength distribution average tends to have a larger value than the duct-average. We note here that, for turbulent flow, the Prandtl relation transforms into the velocity distribution

$$
P(V) d V=2 n X^{n-1}\left(1-X^{n}\right) d X, \quad X=V / V_{c}<1 \quad(V I-6)
$$

with a strongly asymmetric peak at

$$
x_{\max }=\left[\frac{n-1}{2 n-1}\right]^{1 / n}
$$

As was noted for the DP scheme, a careful calibration can translate the wavelength spectrum peak to the true average particle or flow velocity, at least over a certain range of indices $n$. For Newtonian-laminar flow, the velocity has a step-function spectrum,

$$
P(V) d V=d X, \quad X<1 ;
$$

for Pseudoplastic flow,

$$
\begin{aligned}
P(V) d V & =\frac{1-s}{(1-X)^{s}} d X, X<1, \\
s & =\frac{1-m}{1+m},
\end{aligned}
$$

$m=$ Metzner Flow Behaviour Index $(s=0$ for Newtonian, $i$ for plug-flow). Eq. ( $V-9)$ shows an abrupt cutoff at the centerflow velocity while the average gradually increases from 0.5 to 1 ,

$$
\bar{x}=\frac{1}{2-s} \text {. }
$$


SS-Doppler measurements on slurries have shown distributions somewhat resembling step-functions $(\mathbf{V}-2)$. The claim of a patent $(\mathbf{V}-\mathbf{4})$ that the duct-average velocity can be inferred as one-half of the "knee" velocity was evidently based on the supposition, not confirmed by experience, that Newtonian-laminar (or, near-Newtonian-laminar) flow predominates in slurry conveyance.

To sum up, neither one of the SS-acoustic schemes offers a reading accuracy good enough for feed rate control in industrial dense slurry conveyance. Even for turbulent flow of lighter slurries, lack of sensing uniformity, characterizing both schemes, calls for a calibration that, for reasons repeatedly noted in this report, is impractical for either type of slurry conveyor.

\section{(2) Magnet ic FM and Coriolis' Force FM}

The third and fourth schemes, Magmeter(V-5) and Coriolis Flow meter (V-8), are non-intrusive yet manage to sense the entire duct cross section with high uniformity and, moreover. produce readout signals directly proportional to the local velocity; in combination, these two characteristics yield the true average velocity. Magmeters, imposing a uniform magnetic field, respond to the Lorentz force; Coriolis' Force devices stimulate a response through mechanical vibration on a hairpin duct section: both forces are vector cross-products of the stimulant and the medium velocity:

$$
\begin{aligned}
& \vec{F}=2\left[\int q(r) \vec{V}(r) \quad r \quad d r\right] \times \vec{B} \quad(\text { Lorent } z) ; \quad(V I-11) \\
& \vec{F}=4\left[\int \rho(r) \vec{V}(r) \quad r \text { dr }\right] \times \omega \quad \text { (Coriolis) } \quad(V I-12)
\end{aligned}
$$

where $q(r)=$ local free electron density, $\rho(r)=10 c a l$ bulk mass density of the medium. Inherent in the manner in which these schemes produce the average is their independence from the flow mode. Both devices are commercially available from many vendors. Both are based on well-understood principles. Magnetic Induction, the principle on which Magmeters rely, was discovered 170 years ago by Faraday and used by him to measure the flow of the Thames river; the first commercial Magmeters appeared in the early fifties. In 1974, Pulsed-DC Magmeters were introduced, improving sensitivity by a factor of 10 . Unfortunately, DC and Pulsed-DC devices are sensitive to Phase Fluctuation Noise, hence, Magmeters of AC type, immune to Fluctuation Noise, must be used for slurries even though this type of Magmeter does not perform well in laminar mode( $\mathrm{V}-6)$. As the solid fraction increases, medium conductivity declines and signal strength weakens, to the point where the signal-to-noise ratio becomes prohibitively large. Efforts to solve these problems(v-7) are continuing but have not been successful so far.

Coriolis' Force Flow meters(V-8), responding to mass flow, are not affected by non-uniform phase distribution within the duct and certainly operate equally well in conducting and dielectric media. Well-established for about 10 years and offered by a number of firms, these devices are not as yet available (nor likely to be) for ducts of 2-in and larger diameters: severe mechanical problems tend to arise in installing such meters at industrial plant sites. A possible drawback of any Coriolis' Force scheme is the increased pumping power needed to overcome the considerable flow impedance of hairpin or similar configurations (a straight-through device is offered commercially but involves 
bellows mounting of the vibrated duct section, impractical for slurries).

Both Magmeters and Coriolis' Force Flow meters are customarily calibrated; zero shifts of AC-type Magmeters, caused by deposits on electrode surfaces, require occasional trimming. As neither instrument is temperature-sensitive, ambient temperature calibration is permissible in principle; in practice, dense slurry viscosity varies too much with temperature to make such a calibration a viable option.

TABLE VI-2: SPECIAL FLOW VELOCITY MEASUREMENT TECHNIQUES

\begin{tabular}{|c|c|l|l|}
\hline Type of FM & max. duct size & readout & accuracy \\
\hline RPI & unlimited & $\begin{array}{l}\text { single particle } \\
\text { transit time }\end{array}$ & excellent (1) \\
PNA & $\cong 8$ in & $\begin{array}{l}\text { dispersed activity } \\
\text { transit time } \\
\text { excellent (2) } \\
\text { natural phase inhomogeneity } \\
\text { transit time }\end{array}$ & $\begin{array}{l}\text { very poor (3) } \\
\text { of detector }\end{array}$ \\
\hline
\end{tabular}

Notes:

(1) If recommended data processing is used and "hot" particles are accurately fabricated to match entrained solids

(2) good (2-3\% error) for laminar(non-Newtonian) flow, excellent ( $<1 \%$ error) for Turbulent flow

(3) $5-50 \%$ error in reported CCF tests with conventional sensors and data processing methods.

\section{(3) Radioactive Particle Injection and Pulsed Neutron Activation}

The R.P.I (Radioactive Particle Injection) and P.N.A (Pulsed Neutron ACtivation) schemes are both capable of excellent accuracy; they also are both unsuitable for on-line service in industrial plants: aside from reading much too slowly for process control, their cost is very large, equipment is delicate and requires specially trained personnel, finally, both involve a radioactivity hazard. The unusually high accuracy that either scheme is capable of suggests its use for purposes of calibration, in a temporary on-site installation or (preferably) on a Test Loop(*). This is probably impractical today but could become so if adequate planning and resources are provided.

RPI, an outgrowth of earlier salt or dye injection schemes(V-9), requires the fabrication and injection of radioactive particles that simulate the size, size distribution and density of entrained solids. Radioactive halflife should be short enough to render the injected activity harmless in a few days. In contrast to salt/die schemes that require an intrusive conductivity detector or transparent duct and medium, an injection interlock is the only installation needed by RPI. Two gamma detectors, enclosed in suitable shielding, are sited close to the duct, downstream from the injector, at a known mutual distance $Z$. As they pass each detector, "hot" particles produce count rate excur-

(*) Accuracy is excellent only when equipment is installed on a long, straight duct that may well not be available at the intended measurement site. 
sions whose peaks accurately mark the closest approach or moment when the particle passes the detector center. Channel counts are summed over $\cong 1 \mathrm{~ms}$ (during which time particles travel $\leq 0.5 \mathrm{~cm}$ ), and stored. A computer program analyzes these records and determines Upstream and Downstream peak times $\mathrm{TU}_{k}$ and $\mathrm{TD}_{\mathbf{k}}, \mathrm{k}=1,2, \ldots \mathbb{N}, \mathbb{N}=$ count rate excursion (injected particle) number.

Individual particle velocities are grouped in velocity channels to portray the velocity distribution, or simply averaged over $\mathbb{N}$ to obtain the true duct-average particle velocity

$$
\bar{v}=\frac{1}{N} \sum_{k=1}^{N} \frac{Z}{T U_{k}-T D_{k}}
$$

with a statistical error proportional to $1 / \sqrt{ } \mathbb{N}$ and accuracy related to that of the peak timing routine. A RPI run on a test loop that returns each particle within minutes can thus produce measurements with a precision of $1 \%$, yet a feasible number (50-100) of injected particles. As described here, "hot" particle injection differs from the conventional injection of a radioactive solution into a duct carrying a liquid, similar to die or salt-solution tagging $(\mathrm{V}-9),(\mathrm{V}-10)$. Liquid sample injection, described by Allen(V-10) and Hull(V11), has been extensively reviewed by Clayton(V-12), $(V-13)$; "hot" particle injection was described by Sauerwein et al(V-14), Fortier and Chen(V-15) and Dunn and Gardner(V-16). The high accuracy of liquid injection-based flow measurement in either "Integrated Pulse" or "Constant Velocity Injection" mode is well-established. However, recent, more stringent regulations with regard to the disposal of radioactive waste make liquid injection, an irreversible process, less acceptable than the use of retrievable particles(").

The RPI scheme was tested several years ago at ANL, in a series of runs at the GSTF (Gas-Solids Test Facility). To make these measurements, $10 \mathrm{NaI}$ Scintillator gamma detection channels were provided with electronics that allowed count rates in excess of $1,000,000 / \mathrm{sec}$ without pileup loss; lossless transfer to magnetic storage was provided for by means of a shift register-based interisediate multiscaler memory with "Ping-Pong" feed. Particle velocity and local pressure, measured at a number of points downstream from injection, were modeled by differential equations with a particle density-dependent entrainment coefficient. Making use of the ATSR research reactor (conveniently located in the same building as the Test Loop) to activate the "hot" particles, this work involved the collaboration of experts in three disciplines. A comprehensive report describing procedures, electrunics, hardware and results was written but (for various reasons) has remained unpublished.

(*) With either solutions or particles, the radiation hazard must be minimized through use of fast-decaying activities, produced by a nearby accelerator or research reactor. For solids such as ground/crushed coal, individual particles of various sizes are soaked in a radioactive solution and lightly coated with sealant. Mineral particles containing sodium, or glass beads, can be directly irradiated. For particle diameters $\geq 1 \mathrm{~mm}$, a technique developed at ANL, fabrication of particles from epoxy loaded with indium oxide, is practical and yields initial peak count rates per particle of a few hundred thousand/sec. Halflife of the induced activity is $<1$ hour. 


$$
\begin{aligned}
& \text { 震 }
\end{aligned}
$$

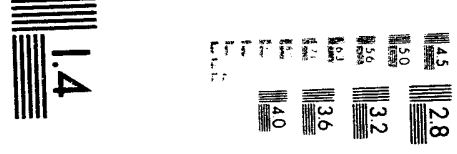

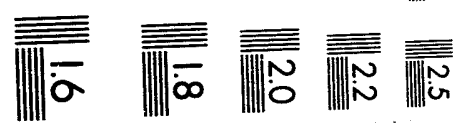



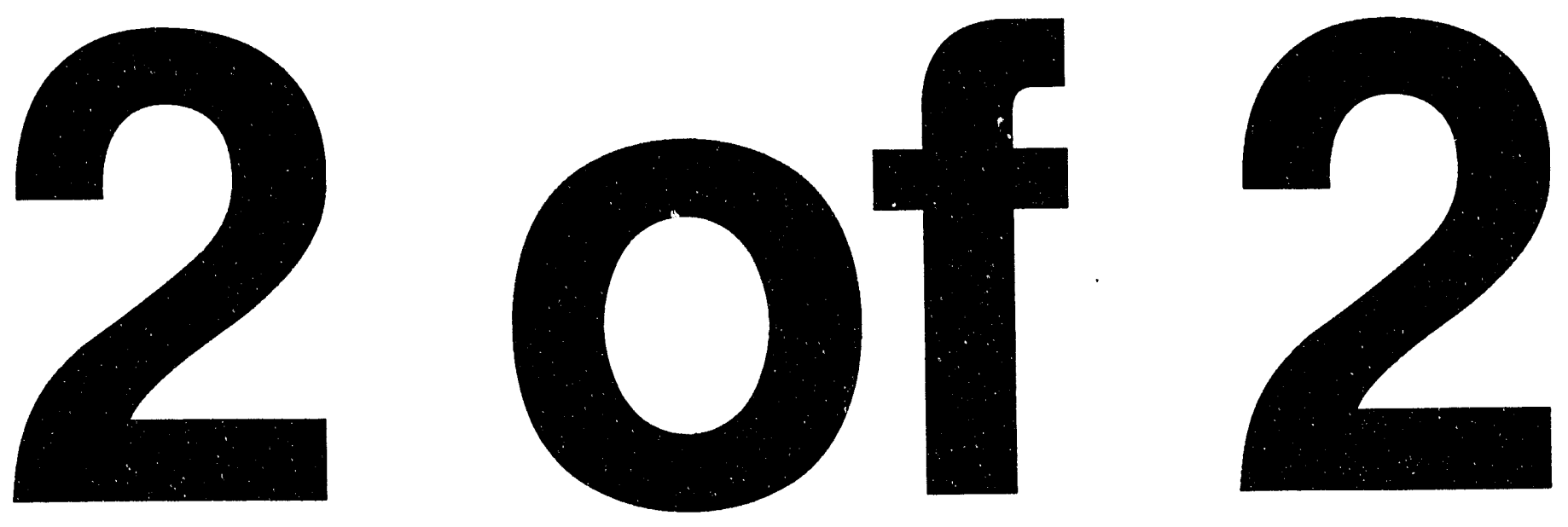
Applicable to entrained-solids flow in any medium and for any duct diameter, RPI produces information without calibration or modeling, on an absolute basis. Pulsed Neutron Activation (PNA) also yields absolute results but, to do so accurately, requires fluid-mechanics modeling (as reviewed in refs. $(\mathrm{V}-17)-(\mathrm{V}-20))$. In lieu of a pair of gamma detectors, PNA uses a pulsed neutron generator upstream and a gamma detector downstream. Generator pulse and detector response counts are acquired in "Multiscaler" format, initiated by the accelerator firing circuit. When a statistically adequate number of counts has accumulated (a process that may take 1-30 min), the profile is processed according to specific programs designed for different flow modes (quasi-turbulent, laminar-Newtonian, Bingham or Pseudoplastic) to extract the duct-averaged velocity as a weighted-integral ratio, corrected for truncation (in the case of laminar flow). As described in the reports cited above, the decay and background-corrected profile

$$
G_{1}=\left(C_{1}-B\right) e^{\lambda t_{1}}
$$

$t_{1}=i \Delta t, \Delta t=$ scaling interval, is summed with weights,

$$
\langle V\rangle=2 \sum_{1} \frac{G_{1}}{t_{1}^{2}} / \sum_{k} \frac{G_{k}}{t_{k}}
$$

for turbulent flow, to yield the integral ratio deduced from modeling,

$$
\langle V\rangle=Z \frac{S G(H, t) d t / t^{2}}{\int G(H, t) d t / t}
$$

where

$$
G(H, t)=\exp -\frac{(Z-\bar{v} t)^{2}}{2 H t}
$$

is the decay-adjusted Taylor-Aris dispersion(V-21), $(\mathbf{V}-22)$, expressed in terms of the time-dilating dispersion parameter $H$. Laminar models are presented in refs. $(\mathrm{V}-17)$ and $(\mathrm{V}-18 \mathrm{a})$. Earlier work $(\mathrm{V}-23)$ made use of Peak offset Timing (P.O.T.) for turbulent flow(*). To compare data processing schemes, a series of careful measurements was made on a Test loop equipped with a full flow-diverter: whereas P.O.T. yielded errors up to $6 \%$, Eq. (VI-16) agreed with the diverter within $0.2 \%$, the reproducibility of the latter.

\section{(4) Cross-Correlation Flow Measurement}

The last method listed in Table VI-2 is generically known as Cross-Correlation, or CCF, Flow metering. CCF and PNA have a number of similarities and a number of differences that are worth pointing out:

(a) The CCF "Readout", the computed Cross Correlation Function, has a characteristic peaked-asymmetric shape. The PNA "Readout", the count rate profile, has a very similar shape. Deterministically, both readouts are Transit

(*) P.O.T., a convenient but inaccurate processing technique, has been widely adopted for Cross Correlation (where it continues to produce large errors). 
Time Distributions convolved with an instrumental response function.

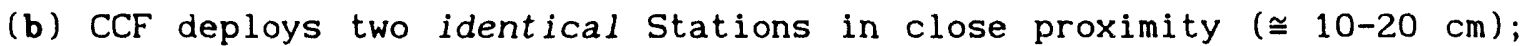
PNA features an Upstream neutron generator/Downstream gamma detector at a mutual distance of $\cong 1 \mathrm{~m}$.

(c) CCF responds to pre-existing natural local inhomogeneities in some bulk variable, carried along with the local stream velocity, hence Signals $U(t)$ and $D(t)$, featuring more or less similar excursions whenever an inhomogeneity passes through the field of view $\Delta z$ of each station, have the same average and variance. The Upstream "signal" of PNA is a delta function, generated by an artificial, Unique event.

(d) Both schemes process by integrating channel Signal samples over successive sampling intervals $\Delta t<\Delta z / V(\Delta z=$ detector station lenght, $V=$ mean flow speed). For PNA, zero time is set by the neutron generator; CCF, monitoring a stationary process, has no zero-time.

The Cross-Correlation Function CCF $(k)$ is computed at successive offsets $t(k)=k \Delta t$ of sample string pairs $\left\{U_{1}, U_{2}, \ldots U_{N}\right\},\left\{D_{1}, D_{2}, \ldots D_{N}, \ldots D_{N+M}\right\}$ and their averages $\langle U\rangle$ and $\langle D\rangle$ :

$$
\operatorname{CCF}(k)=\sum\left[U_{1}-\langle U\rangle\right]\left[D_{1+k}-\langle D\rangle\right], \quad k=1,2, \ldots M \quad(V I-18)
$$

In treatises on cross-correlation(CC-1a), (CC-1b), this is usually presented in alternative integral forms,

$$
\operatorname{CCF}(\tau)=\frac{1}{T} \int_{0}^{T} u(t) d(t+\tau) d t=\frac{1}{T} \int_{0}^{T} u(t-\tau) d(t) d t \quad\left(V I-18^{\prime}\right)
$$

where functions $u, d$ are implicitly assumed to be zero-average, AC signals (as encountered in signal communications and allied fields of Electrical Engineering ).

Cross Correlation processing per se is a widely used stratagem that has been variously applied in nuclear reactor kinetics, acoustic noise and vibration diagnostics, seismic studies, signal communications and other fields, often just to establish that a correlation, indicating a problem of some sort, exists, or is relatively stronger for some specific pairing of signal channels than for another. For flow velocity measurement, accurate rather than qualitative information is needed and must be extracted from Eq. (VI-18); as already mentioned, this has not been successful. The lack of accuracy of conventional CCF flow measurement invites comparison with PNA, whose accuracy is wellestablished, with due recognition of the similarities and differences pointed out in the foregoing. At the same time, a discussion of conventional CCF velocity measurement data processing methods is in order. As those methods developed over a long time while means of computation underwent a revolutionary change, this discussion, introducing the more detailed treatment of CCF in the next section, takes the form of a historical review. 


\section{(C) Cross-Correlation Velocity Measurement}

\section{(1) Historical keview and present Status}

The data acquisition and processing methods now almost universally used for Cross Correlation flow measurement are not based on modeling but, instead, gradually developed in the course of a long history of almost 50 years. The following brief review of this history should put the matter of data processing into context.

When Cross Correlation was first applied to Flow velocity measurement in 1946, signals were produced with chart-recorders while multiply-add operations were executed by hand, on mechanical calculators(CC-2). The amount of labor this entailed precluded a sample number that could make statistics better than barely adequate, let alone a number of time-offset points that could encompass the entire transit time distribution. Given those circumstances, it was just possible to produce a rough plot of a few CCF points in the vicinity of the peak. Thus, P.O.T., reading the Peak offset Time by "eyeballing", was the only practical means of data processing. Subsequent reports on velocity measurements by CCF, appearing at a rate of one or two per year, fall into two categories: Emulating Mitra's measurement of ionospheric velocities(CC-1), the scheme was occasionally applied as a method of last resort, for scenarios where no alternative was available; principally, "feasibility studies" were reported, harnessing a variety of fluctuating bulk variables and sensing devices to the task. In addition to acoustic wave, light and gamma beam transmission, EM Field and Microwave sensors, responding to phase fluctuations, sensors responding to temperature, electric charge, magnetic susceptibility and fluorescence fluctuations were investigated. Neither type of report usually concerns itself with error analysis. Where a means of comparison, such as a Diverter, was available, large errors were invariably found but treated (in the traditional mode of instrumentation engineering practice) as "meter factors". Results were thus judged satisfactory if a straight line, indicating a "linear" response, could be drawn through data points (linearity being a specific advantage in calibration since only a few points need to be measured to establish a slope).

As computers began to be available for research activities and off-line CCF computations became feasible, the cost of computer time still at first militated against computation of the CCF at a large set of points(CC-2). The discovery of the FFT scheme(CC-3)-(CC-7), drastically reducing the number of multiplications needed to execute a Fourier Transform, gave impetus to more detailed off-line Correlation processing for various purposes, including Flow Velocity measurement(CC-8) (for on-line flow measurement, FFT, requiring a full-size digital computer, was both too costly and too slow). Several research groups were thus led to design and construct simple and relatively inexpensive Correlators based on Parity Correlation(CC-9), which can be implemented with sets of fast-logic ENOR(CC-10). As the CCF peak shape is strongly distorted by Parity Correlation(CC-11), P.0.T. still remained the only data processing option for those devices, trading accuracy for speed. The advent (some 10 years ago) of the Booth's Algorithm MAC (Multiply-Accumulate Chip) (CC-12) obsoleted Parity Correlation. Pari passu, the development of small computers of PC type, equipped with additional slots for interface and high- 
arithmetic hardware, obsoleted FFT processing: Direct execution of Eq. (V-18) can now be implemented with equipment costing less than $\$ 2000$, producing a 100-point Cross Correlation Function for $2 \times 4000$ samples in 16 milliseconds. As direct computation does not distort the shape, it allows the possibility of model-based data processing and thus may finally obsolete the inadequate but well-entrenched P.0.T. scheme of velocity estimation.

\section{(2) Underlying Processes and Data Processing in CCF and PNA}

Cross-Correlation is treated not only in textbooks on Statistics, but has become a topic for texts(CC-13), monographs(CC-14) and survey articles(CC-15), (CC-16) in various branches of engineering. To achieve generality, formal discussions of engineering applications of Cross-Correlation(CC-15) represent specific objects (the duct and its flowing contents) about which information is required as unspecified Dynamic Systems with Signal Input and Signal Output ports (other examples of "Dynamic Systems" are structures, mechanisms, electrical circuits or, say, currency exchanges). The relation between an arbitrary signal $x(t)$ applied to the Input and the signal $y(t)$ appearing at the Output port is described by

$$
y(t)=\int g\left(t^{\prime}\right) x\left(t-t^{\prime}\right) d t^{\prime}
$$

if the Output signal is noiseless and the System linear (neither of which is necessarily the case). Integration runs from 0 to $\infty$ and $g\left(t^{\prime}\right)$ is the System Response, the information sought by many applications of the Correlation technique. To obtain it, one can in principle "stimulate" the System with a delta function $x\left(t-t^{\prime}\right)=\delta\left(t-t^{\prime}\right)$ (e.g., produce a foreign exchange price list by converting one dollar at a time). In many applications, this is not practical. A second method is to pass the Output through a special reversing filter; unfortunately, this Matched Filter scheme tends to be just as impractical. The third (and only practical) method involves the Autocorrelation Function (ACF) and Cross-Correlation (CCF) Functions of $x(t)$ and $y(t)$,

$$
\operatorname{ACF}(\tau)=\frac{1}{T} \int_{0}^{T} x(t-\tau) x(t) d t ; \quad \operatorname{CCF}(\tau)=\frac{1}{T} \int_{0}^{T} x(t-\tau) y(t) d t .
$$

Applied to the foregoing response integral, Eq. (VI-19), one finds that

$$
\operatorname{CCF}(\tau)=\int g\left(t^{\prime}\right) \operatorname{ACF}\left(\tau-t^{\prime}\right) d t^{\prime}=\int g\left(\tau-t^{\prime}\right) \operatorname{ACF}\left(t^{\prime}\right) d t^{\prime} \quad(V I-20)
$$

from which the response can be extracted by de-convolution. These relations are supposed to apply in general. For the specific case of a flowing slurry viewed by two sensors, ref. (CC-15) argues, on the basis of note (a) of the foregoing comparison to PNA, that the System Response $g(\tau)$ can be interpreted as the Transit time distribution $W(\tau)$, also pointing out that sensing bias may in practice distort the CCF relative to the true transit time distribution (but, unfortunately, overlooking the possibility of applying PNA Moments Ratio data processing to CCF flow measurement).

Now, $g(\tau) \equiv W(\tau)$ in the limit of an ACF narrow enough to be fairly represented by a delta function - in which case the CCF becomes the transit time 
distribution(CC-17). However, for typical EM Field sensing electrodes, a delta-function approximation of the ACF would not be very accurate. Besides, there is some question exactly what transit time distribution is implied. Finally, differences between delta-function tag injection and random fluctuations in the phase distribution, pointed out in notes $(b)-(d)$, certainly could invalidate or modify the identification $g(\tau) \equiv W(\tau)$.

The essential difference between PNA and CCF, revealed in points (b)-(d), is one of underlying assumptions: PNA, injecting (in effect) a liquid tag, assumes rapid radial and axial dispersion according to the Taylor model; yet, that assumption may not be appropriate for particles and clusters (especially somewhat larger particles that yield substantial CCF signals). As entrainment is less effective for large particles, one would not be expect them to be engaged by large and small turbulent eddies and thus to disperse as rapidly as a liquid tag but rather, to follow a more staid trajectory - at least over the relatively short $(\approx 10 \mathrm{~cm})$ distance between CCF stations. This is also suggested by the persistence of phase inhomogeneity patterns and clusters, without which there would scarcely be sufficient correlation to make CCF work at all.

These considerations suggest a model that assumes:

- individual particle (and cluster) velocities remain quasi-constant while traversing the relatively short distance between the stations. The velocity (and Transit time) distribution thus measures the number of inhomogeneities that have certain local velocities.

- the mean distance between such inhomogeneities per unit duct length is large in comparison to the station length $\Delta z$.

- a unit step sensor response while a unit inhomogeneitiy is present in the field of view, of length $\Delta z$, of each detector.

- Negligible inhomogeneity length, $\delta z \ll \Delta z$.

For the first exercise, we shall also assume

- Uniform velocity $V$, represented by the distribution function

$$
W\left(t^{\prime}\right)=\delta\left(t^{\prime}-Z / v\right) \text {. }
$$

Putting $T=Z / V$ and integrating from 0 to $\infty$,

$$
\begin{aligned}
& \int W(t-\pi) d t=1, \\
& \int W(t-\pi) G(t) d t=G(\pi),
\end{aligned}
$$

hence,

$$
\int W(t) G(t-\tau) d t=G(T-\tau)
$$

for any function $G(t)$. Let 


$$
\begin{aligned}
\Delta t & =\text { sampling interval, } \\
Z & =\text { sensor mutual separation, } \\
\mathbb{K} \Delta t & =\mathrm{Z} / \mathrm{V}, \\
\mathrm{K} \Delta \mathrm{t} & =\Delta \mathrm{Z} / \mathrm{V}, \\
\mathbb{N} \Delta \mathrm{t} & =\mathrm{T}=\text { averaging base }(\mathbb{N}=\text { number of samples processed }), \\
\mathrm{M} & =\text { mean number of inhomogeneities in duct length } \mathbb{R}=\mathrm{V} \mathbb{T}, \\
\mathrm{S} \Delta \mathrm{t} & =\text { mean time between inhomogeneities; } \mathrm{MS}=\mathbb{N} .
\end{aligned}
$$

\section{Signals}

$$
U(t)=C_{u}^{\prime}(t) / C_{u}-1, \quad D(t)=C_{d}^{\prime}(t) / C_{d}-1
$$

consist of steps (unit height, duration $T$ ) in random sequence, zero between steps: $C_{u}^{\prime}, C_{d}^{\prime}=$ sensor response in the presence, $C_{u}, C_{d}$ in the absence of an inhomogeneity (note that, for purposes of distinguishing between inhomogeneities (relatively sparse) and solids per se, measurements $C_{u}, C_{d}$ include homogeneous solids). Averaging over $\pi,\langle U\rangle=\langle D\rangle=K / S$. Let

$$
\begin{gathered}
U_{1}-\langle U\rangle=u_{1}, D_{1+k}-\langle D\rangle=d_{1+k} \\
u_{1}, d_{1+k}=1-K / S \text {, particle present, }=0 \text {, particle absent. (VI-23) }
\end{gathered}
$$

For this model, the kth Cross-Correlation Function term comes to

$$
\left.\begin{array}{rlr}
\operatorname{CCF}(k) & =\frac{1}{\mathbb{N}} \sum\left[u_{1}\right]\left[d_{1+k}\right] & \\
= & & \\
= & k-K<-K \text { and } k-K>+K, \\
= & -K<K-K<0, \quad(1-K / S)^{2}[K-(K-K)] & 0<k-K<+K .
\end{array}\right\}(V I-24)
$$

Eqs. (VI-22) describe a Response Function $F(k-K)=F(t-\pi), t=k \Delta t, \mathbb{T}=K \Delta t$, in the shape of an equilateral triangle rising to $(K / S)(1-K / S)^{2}$ at time $\pi$, with base length $2 \mathrm{~T}$ and area $(\mathrm{KT} / \mathrm{S})(1-\mathrm{K} / \mathrm{S})^{2}$. To simplify the following discussion, the factor $(1-K / S)^{2}$ is set equal to unity, supposing that

$$
K / S=M T / g=\text { "duty factor" }<1 .
$$

The Autocorrelation Function $A C F(k)$ for this model is readily found by analogy with the foregoing, 


$$
\begin{aligned}
\operatorname{ACF}(k) & =\operatorname{CCF}(k, K=0) & \\
& =0, & k<-K \text { and } k>K, \\
& =\left(\varepsilon^{2} / S\right)(i-K / S), & -K<k<0, \\
& =\left(\varepsilon^{2} / S\right)(1-K / S), & 0<k<K,
\end{aligned}
$$

again for a delta-function velocity distribution. Eqs. (VI-24) and (VI-25) indicate that the CCF is the ACF shifted by $K \Delta t$, as also predicted by Eq. (VI-20) when the velocity distribution Eq. (VI-21) is substituted for $g(t)$ (as suggested by ref.(CC-15)). Both CCF and ACF are symmetric with respect to timereversal; neither is a delta function.

Following up the example of PNA data processing discussed in the preceding, we shall explore the possibility of velocity computation by means of moment ratios in which we may represent the transit time distribution by the CCF :

$$
\mathcal{R}_{1}=M_{1} / M_{1-1}=\frac{\operatorname{SCCF}(t) \mathrm{dt}[Z / t]^{1}}{\operatorname{SCCF}(t) \mathrm{dt}[z / t]^{1-1}} .
$$

Converting Eqs. (VI-24) to time functions, we find, for example,

$$
\begin{aligned}
M_{1}=z \int C C F(t) d t / t & =\frac{M Z}{N \Delta t}\left[\int_{\pi-T}^{T}[t+T-\pi] d t / t+\int_{\pi}^{T+T}[\pi+T-t] d t / t\right] \\
& =\frac{V T^{2}}{S \Delta t}[(1-x) \log (1-x)+(1+x) \log (1+x)]
\end{aligned}
$$

where $x=T / T=\Delta z / Z$. Expanding the logarithmic terms, one readily obtains

$$
Z \operatorname{SCCF}(t) d t / t=\left(V^{2} / S \Delta t\right)\left(1+x^{2} / 6+x^{4} / 15 \ldots\right),
$$

hence,

$$
\mathcal{R}_{1}=\frac{2 \int \operatorname{SCF}(t) d t / t}{\operatorname{SCCF}(t) d t}=\left(\frac{V T^{2}}{S \Delta t}\right)\left(\frac{S \Delta t}{T^{2}}\right)=v .
$$

Any other moments ratio turns out to yield the same result; equally accurate (and, of course, easier) is the traditional procedure of plotting the CCF and reading the P.O.T. (Peak Offset Time) by "eyeball" or Savitzky-Golay smoothed differentiation.

To sum up these exercises of the one-velocity model: Eq. (VI-20) applies, moments-ratio modeling likewise applies. Proceeding by small increments, we next introduce a two-velocity model $\left(V_{1}, V_{2}\right.$ with weights $\left.m_{1}, m_{2}\right)$ :

$$
W(t) d t=m_{1} \delta\left(t-\pi_{1}\right)+m_{2} \delta\left(t-\pi_{2}\right)
$$


where

$$
m_{1}=M_{1} /\left(M_{1}+M_{2}\right), \quad i=1,2
$$

are normalized weights, $M$ are numbers as in the foregoing. The wejghted average velocity $\langle V\rangle$ for this model is

$$
\langle V\rangle=m_{1} V_{1}+m_{2} V_{2}
$$

the CCF consists of two triangular peaks centered at $\pi_{1}$ and $\pi_{2}$, of widths $T_{1}, T_{2}$ and heights $m_{1} K_{1} / N, m_{2} K_{2} / N$ :

$$
\begin{aligned}
& \operatorname{SCCF}(t) d t=(1 / \mathcal{T})\left[M_{1} T_{1}^{2}+M_{2} T_{2}^{2}\right]=\left(\Delta z^{2} / \mathcal{T}\right)\left[M_{1} / V_{1}^{2}+M_{2} / V_{2}^{2}\right] ; \quad(V I-29) \\
& R_{1}=\frac{Z \operatorname{SCCF}(t) d t / t}{\operatorname{SCCF}(t) d t}=\left(1+x^{2} / 6 \ldots\right) \frac{m_{1} / V_{1}+m_{2} / V_{2}}{m_{1} / V_{1}^{2}+m_{2} / V_{2}^{2}} \cdot \quad\left(V I-27^{\prime}\right)
\end{aligned}
$$

where the $x^{2}$ term can be dropped for $\Delta z / Z \ll 1$. Eq. (VI-27') implies that $\mathcal{R}_{3}$, effectively raising the power of $V$ by 2 , is the moments ratio we seek. The second and third moments are readily calculated,

$$
\begin{aligned}
& M_{2}=\int \operatorname{CCF}(t) d t[z / t]^{2}=\frac{\Delta z^{2}}{\mathfrak{T}}\left(1+x^{2} / 2+x^{4} / 3 \ldots\right) ; \\
& M_{3}=\int \operatorname{CCF}(t)[z / t]^{3}=\frac{\Delta z^{2}}{g} \frac{M_{1} V_{1}+M_{2} V_{2}}{1-x^{2}},
\end{aligned}
$$

hence, dropping terms of 4 th order in $\Delta z / Z$,

$$
R_{3}=\frac{Z^{3} \int\left(\operatorname{CCF}(t) d t / t^{3}\right.}{Z^{2} \int C C F(t) d t / t^{2}}=\frac{m_{1} V_{1}+m_{2} V_{2}}{1-x^{2} / 2}
$$

which, aside from the small factor $x^{2} / 2$, agrees with Eq. (VI-28).

Eq. (VI-30) is readily generalized to any transit time distribution $W(t)$ that can be represented by a finite number $Q$ of delta functions,

$$
W(t) d t=\sum_{j=1}^{Q} m_{j} \delta\left(t-T_{j}\right) .
$$

The duct-average velocity can thus be accurately calculated from an undistorted CCF measurement, whereas the P.O.T. method is clearly incapable of accuracy for any velocity distribution that differs significantly from the one-velocity model.

Note further that transit time distribution (VI-31) yields an ACF that is 
strictly symmetric as long as inhomogeneities remain invariant over a time span within which the ACF has a significant magnitude, while the CCF is typically skewed for all transit time distributions that are produced in ducted flow. The functions can be expressed in terms of their single-velocity components $A_{k}$ :

$$
\begin{aligned}
\operatorname{CCF}(t)=\frac{1}{\mathbb{N}} \sum m_{k} A_{k}\left(t-T_{k}\right)=\frac{1}{\mathscr{T}} \int d t^{\prime} \sum m_{k} A_{k}\left(t-t^{\prime}\right) \delta\left(t^{\prime}-T_{k}\right) ; & (V I-32) \\
\operatorname{ACF}(t)=\frac{1}{N} \sum m_{j} A_{j}(t) & (V I-33)
\end{aligned}
$$

which evidently disagrees with Eq. (VI-20). The reason for that disagreement lies in the assumption of Eq. (VI-20) of an ACF (1.e., function A) whose shape does not vary with the velocity, whereas in the model used here the ACF base width scales with $\Delta z / V$. Admittedly, this model still is rudimentary; a more complex model could introduce clusters of varying length and persistence (fading). Unfortunately, no modeling at all seems to have been tried in either recent or earlier reports in the CCF Flow measurement literature; at the same time, not many reports describe measurements that extend over a significant velocity range. Moreover, such measurements as have been reported invariably used biased sensing(*). While the question whether Eqs. (VI-32) and (VI-33), or Eq. (VI-20), are better representations of $\operatorname{CCF}(t)$ and ACF $(t)$ cannot be convincingly answered, there is little doubt concerning the improved accuracy of Moments Ratio data processing, if and when that method is applicable.

Difficulties with the moments-ratio scheme arise if, for example, very long density excursions, simultaneously present in both stations, are produced by pump action and yield finite values for $W(t)$ at or below $t=0$. Other problems are discussed further on. The moments-ratio fails altogether if $W(t)$ does not decline faster than $1 / t$ with increasing $t$. As a result, laminar flow, which features such a distribution, cannot make use of the Moments Ratio. How that problem can be dealt with is discussed below.

It is worth stressing again that, to make effective use of Eq. $(\mathrm{V}-30)$, two requirements must be satisfied by the instrumentation:

- unbiased sensing of the entire duct,

- channel readouts undistorted by electronic filtering of the analog signals delivered by the detectors.

With regard to sensing bias, the literature includes reports on many different sensors, configured in various geometries. Unfortunately, as already noted, whether EM Field interrogation, or acoustic beam transmission, or some other scheme was used, close-to-uniform sensing has been the exception rather than the rule.

(*) unfortunately, that seems to be the case for the large number of reports by the University of Bradford, one of the groups that have especially concentrated on CCF velocity measurement(CC-18) - (CC-21); likewise, the Delft Institute group (CC-22). 
Filtering, a standard and indeed useful ploy when a narrow-band signal is masked by broad-band noise, has of ten been misapplied to produce sharper CCF peaks (whose offset is then more easily read in the classic "eyeballing" P.O.T. mode). Inevitably, this discards the information contained in the original peak shape. Thus, heavy-handed filtering and P.O.T. data processing may well have further worsened the error already produced by biased sensing in a number of CCF velocity measurements.

To test the Moments Ratio computation procedure, it would be clearly desirable to construct a square or rectangular test loop and/or test section that can be inserted into a basic loop of circular cross section. Having thus achieved an unbiased, whole-duct measurement capability, the foregoing model, or more sophisticated models, can be applied to measurements made with appropriately designed electronics. In the absence of a Test loop, a Monte Carlo simulation of turbulent/transition flow may be feasible (the simulation would, of course, not need to be restricted to square duct shape). Until either is done, the only certainty about CCF flow velocity measurement is that the conventional data processing method, aggravated by conventional, heavily biased sensing and filtering, cannot and will not work well enough to make its application in industry practical.

\section{(3) CCF Computation in Detail}

\section{(a) Hardware Considerations}

To consider the detailed means of CCF calculation, we shall assume that sensor analog voltages, suitably amplified, are sampled and digitized at the rate $1 / \Delta t$ until a large number $\mathbb{N}$ of samples (say, 4096), consisting of Fixedpoint, positive integers of 24 or 32 bit length, is on hand. The averages $\langle U\rangle$ and $\langle D\rangle$ of these $2 \mathbb{N}$ samples (covering the readout interval $\mathbb{N} \Delta t=\pi$ ) are computed. Averages are updated every time a new sample arrives, and thereupon subtracted from it, yielding a fixed-point integer \& sign of 2 byte size $(-127$ to +128 ). At the end of another interval $\pi$, a set of 4096 pairs $\delta U, \delta D$ of these numbers is ready for the CCF computation according to Eq. (VI-18).

The conventional alternative to this sample setup routine has long relied on the use of capacitive coupling in the analug electronics to "automatically" produce zero-average analog voltages before sampling and digitizing. However, a sudden change in the mean channel voltage, say due to a flow blockage, pipe burst or other untoward incident, can drive the stage following the coupling capacitor into saturation until the capacitor has had time to discharge through its associated resistor: meanwhile, the channel is paralyzed. Replacing the coupling capacitor with the described digital computation eliminates this hazard and also improves long-term channel stability.

Sample strings are batch-transferred to a board equipped with FIFO-configured, Dual-Ported SRAM, a MAC with Barrel Shifter and other logic. The basic configuration of this board is shown in FIG (VI-2), omitting details that may vary considerably, to illustrate the calculational procedure: 
The computation cycle starts with $\mathbb{N}$ Upstream and $\mathbb{N}+\mathbb{M}$ Downstream samples ( $M$ = number of CCF points) loaded into the 3 FIFO blocks U, D1 \& D2. A $25 \mathrm{MHz}$ Clock, advancing samples along both FIFO chains, successively cycles them throug! the $U$ and D MAC input ports. The MAC forms their product and adds this to the contents of the product sum store or Accumulator. After $\mathbb{N}$ clock cycles, the entire U-block has passed once through the MAC and is now in its original location. At this point, the clock is stopped; one sample is transferred from D1 to D2; the sample formerly occupying the last (front) bin of D2 is discarded. MAC Accumulator contents are transferred to the Shifter; the Accumulator is cleared, the Shifter passes on a downshifted CCF point to the CCF store. This takes an estimated 4 clock cycles, whereupon the $25 \mathrm{MHz}$ clock is enabled and another CCF point is computed, until the D1 store has been emptied. The time required for one complete CCF computation comes to

$$
(4096+4) \times M / 25=164 M \mu s .
$$

The CCF point store now contains the complete CCF. An interrupt is issued to the main CPU; the CCF store contents are transferred to the CPU-accessible main RAM memory and converted to Floating-Point format for further processing (that may occupy the CPU \& resources for a good part of the time remaining to the end of interval $\pi$ ). Concurrently, 4096 fresh samples are received and trimmed. At $\mathbb{T}$, the average velocity is announced; the new sample strings are read into the U1, D1 and D2 stores $\Rightarrow$ the cycle recommences.

\section{(b) Numerical Examples; comparison to FFT}

The process described in the preceding section will now be applied to a turbulent slurry flowing at, say, $\langle V\rangle=5 \mathrm{~m} / \mathrm{s}$. Choosing $Z=$ distance between sensor stations $=10 \mathrm{~cm}$ and $\Delta z=$ station length $=2.5 \mathrm{~cm}, \mathrm{f} \cong \Delta z /\langle V\rangle \Delta t=$ number of "frames" (in the moving picture analogy of this process) during which a point of the medium moving at the velocity $\langle V\rangle$ remains in the field of view of the detector station. For the chosen set of parameter values, $f=10$. Let $Z=$ distance between stations $=15 \mathrm{~cm}$ then $F=60$ frames pass while a point starting with velocity $\langle V\rangle$ at the center of the $U$-sensor reaches the center of the D-sensor.

To estimate the number of frames $M$ required for the CCF computation, we make use once more of the Transit time distribution generated by the Prandt 1 equation,

$$
\begin{aligned}
W(\tau) d \tau & =\left(1-1 / \tau^{n}\right)\left(2 n d \tau / \tau^{n+1}\right), \\
\tau & =t / t_{c}=v_{c} t / Z,
\end{aligned}
$$

let $T=$ ratio of the transit time within which $99 \%$ of the medium passes between the two detectors to the minimum (centerflow) transit time:

$$
0.99=2 n \int_{1}\left(1-1 / \tau^{n}\right) d \tau / \tau^{n+1}=1-2 / T^{n}+1 / T^{2 n} ; \quad 1 / T^{n}=1-\sqrt{0.99} .
$$

Let $n=6$, appropriate for a slurry, then $T=2.4173$,

$$
\begin{gathered}
V_{c}=1.30<V>=6.50 \mathrm{~m} / \mathrm{s}, \quad t_{c}=20.31 \mathrm{~ms}, \quad F_{c}=t_{c} / \Delta t \cong 47 \text { frames; } \\
t(0.99)=T \times t_{c}=49.10 \mathrm{~ms}, \quad F(0.99) \cong 100 \text { frames. }
\end{gathered}
$$




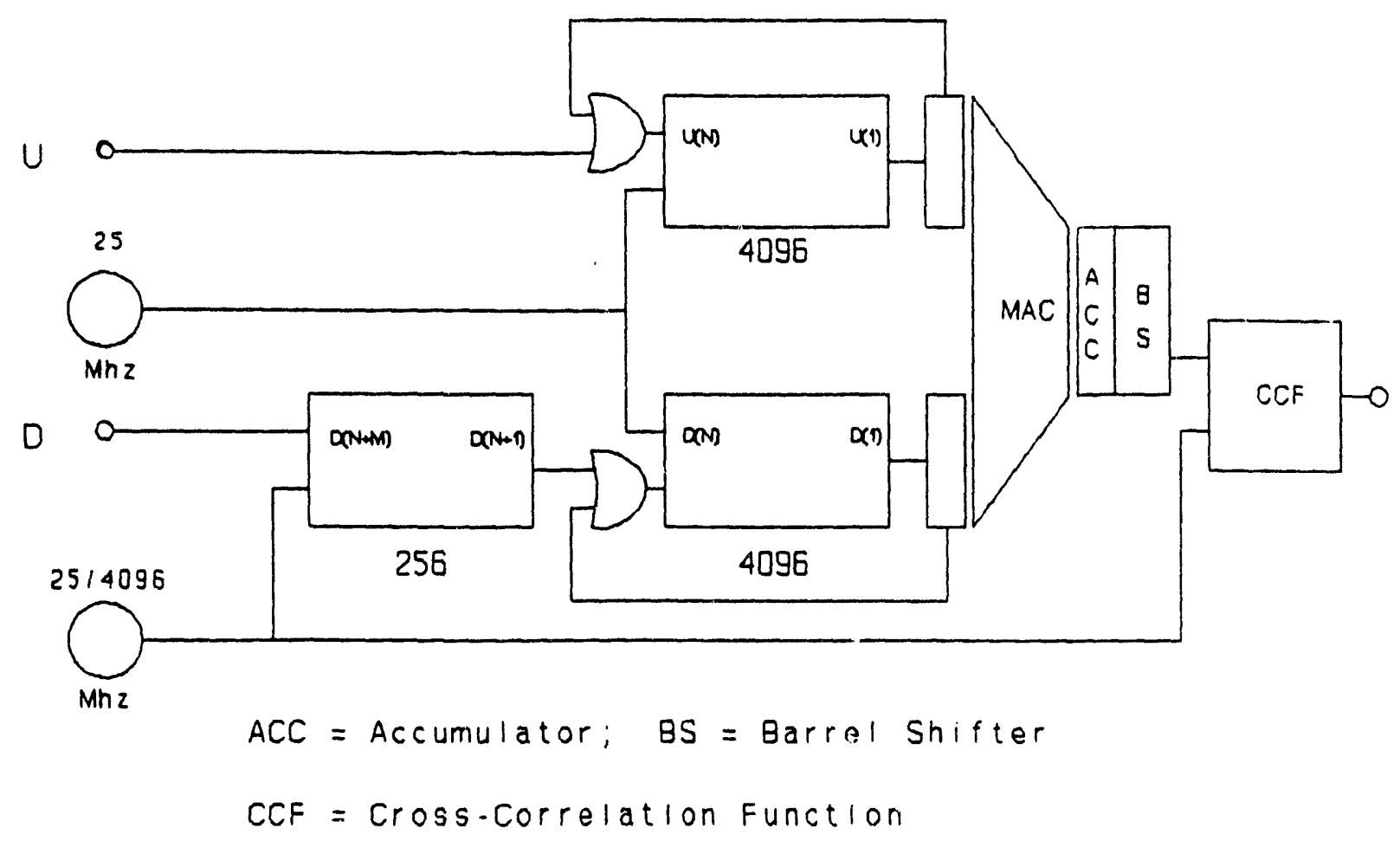

FIG (VI-2) CCF Computation Hardware, schematic

This suggests that for this turbulent flow model, a number $M=F(0.99)+$ $f=110$ should be sufficient; $M=128$ better, 256 ample. The assumption underlying the foregoing estimate is that particles and clusters move in an axial direction and at the velocity indicated by the Prandtl distribution; in fact, turbulence redistributes lighter particles fairly rapidly, necessitating short correlation base paths $Z$ and even shorter station lengths $\Delta z$. This becomes awkward for a pipeline conveyor of, say, $50 \mathrm{~cm}$ diameter: particles must be large indeed to cause a detectable excursion when the plate configuration has a capacity of less than $0.2 \mathrm{pf}$. To compensate for this, $4 \mathrm{k}$ samples are in play; if this is insufficient, the readout interval $\pi$ can be lengthened from 2 to 4 seconds, making $8 \mathrm{k}$ samples available for correlation.

Consider, now, the FFT alternative: starting again with $2 \times 4096$ samples, $2 \times 12 \times 1024$ multiplications (plus other operations), all in Floating-Point, are required to convert from real-time to 2048-point frequency spectra. The spectra are convolved - 2048 more multiplications - a final transform back to the time regime produces the CCF (at rather more points than needed): another 24576 multiplications, or, a total of 39,000. A 38076 co-processor can execute one floating-point multiplication in something like $1.5 \mu \mathrm{s}$. Thus, just the multiplication component of FFT computations takes $58 \mathrm{~ms}$, almost times longer than the direct 128-point calculation. Actually, multiplication time is no longer indicative of total computation time; Overhead in the FFT Butterfly routines must be accounted for as well. Special FFT chips can reduce Overhead 
and execute Multiplications in Fixed-Point, however at a sacrifice of accuracy: FFT processing, no bargain for speed, it also is error-prone. An advantage has been claimed for inferring the velocity from the slope of the complex phase (CC-8); however, as the phase has a unique slope only for a unique velocity or symmetric (quasi-Gaussian) velocity distribution, this scheme is as inaccurate as inferring the velocity from POT, or FFT processing.

FFT has become indispensable in LDA, FTIR and other highly sophisticated instrumentation systems; it is widely applied in Communications, Filtering and a variety of useful tasks; however, so far as CCF velocity measurement is concerned, the foregoing discussion leads to but one conclusion: for that particular task, FFT is the wrong choice(").

\section{(c) Calculation of the Average Velocity, Turbulent Flow}

We now briefly reconsider the calculation of $\langle V\rangle$ from a measured CCF. Eq. $(V-30)$ shows how this can be done, as the ratio of the third to the second moment of $\mathrm{z} / \mathrm{t}$. How well the moments ratio succeeds depends on how accurately the instrumentation can reproduce the CCF shape. Concerning the latter, it has been pointed out that a narrow function $\mathbf{A}(t)$, approaching a delta-function, will produce a $\operatorname{CCF}(t)$ that closely resembles $W(t)$.

Realistically, a minimum sensor width is needed to secure a healthy signal/noise ratio. The model used in the preceding did not take the finite size of particles and clusters into account; in a real slurry, these may be fairly large. The resultant broadening of the function $A(t)$, hence, $\operatorname{CCF}(t)$, relative to $W(t)$ is still symmetric, hence does not shift the moments ratio. A more difficult CCF data processing problem is caused by the cluster "halo" effect: in Astrophysical cluster formation processes that may be an appropriate analogy here, the immediate surroundings of clusters tends to be depleted of mass, producing "halo" regions of less than average solid concentration. As such a structure moves through each sensor station, the ACF (response) function acquires a pattern of correlation and anticorrelation peaks and valleys. Negative (anticorrelation) oscillations convolve with the $W(t)$ function to produce a CCF that also contains negative regions (this effect, though having a physical origin, superficially resembles a $\sin (\tau) / \tau$ peak shape, one of the effects of heavy filtering).

Halo and long-cluster structures constitute a special topic of Multiphase Fluid Mechanics that has not been sufficiently investigated; consequently, there is no model which can be used in applying Eq. (VI-30) to slurries where such structures are important. To proceed, it may be necessary to de-convolve the transit time distribution and response function components, modeling the latter to resemble the shape of the Autocorrelation Function, or modeling the halo effect (which can be calculated only by making a number of ad-hoc assumptions).

( Nevertheless, it is still occasionally rediscovered; thus, a recent engineering report on CCF velocity measurement hails FFT as a "breakthrough". 


\section{(d) Laminar Flow.}

The foregoing integral ratio calculation of the average velocity fails for laminar flow: as the transit 'ime distribution declines with $1 / t$, its tail becomes too long for processing by the moments ratio scheme (even 500-frame CCF processing neglects much of the long-transit peripheral flow). Moreover, clusters that move near the wall will dissolve before reaching the downstream sensor, while centerflow clusters will tend to survive. As a result, the result must be biased, however uniformly the stations sense the duct. Yet, laminar flow is what one must expect in dense-slurry feed lines, and thus cannot be neglected. In this quandary, a peculiarity of laminar flow that was noted in Chapter II can be exploited. To restate this observation,

(a) for every flow pattern that features certain symmetries, there exists a contour along which the local velocity just equals the duct-average,

(b) over a range that encompasses slurry compositions one would expect to find in feedlines, that contour varies remarkably little with the Metzner Flow Behaviour Index $n$, cf. FIG(III-3) and (III-4).

It is now further noted that the region within a duct sensed by EM Field electrodes is defined by the electrode surfaces on one hand, and those field lines that just reach the signal electrode edge, on the other hand. The sensed region can thus be adjusted by introducing electrodes at certain potentials and arranging the geometry so as to distort the edge field lines in a desired manner. Secondly, we note that, if the Upstream and Downstream sensors are configured to sense different, partly overlapping regions, only those phase fluctuations (particles/clusters) that move along the overlapping region will contribute to the CCF.

Combining these observations, it appears that a CCF that represents a narrow velocity spectrum in the immediate vicinity of the average velocity can be selected by a judicious manipulation of field lines. The velocity distribution that characterizes flow within the selected region can be fairly represented by a single delta-function, $W(t)=\delta\left(t-t^{*}\right)$, where $t^{*}=Z /\langle V\rangle$. A mere 20 points should be sufficient for accurate peak location by means of SavitzkyGolay smoothed differentiation. Neither oscillations, nor overlap of $t=0$, will affect the calculation.

A preliminary investigation of Field Line adjustment was made, for a square duct and without taking the dielectric constant of the lateral wall into account(*). Equipotentials and Field lines were calculated for a number of configurations of edge-protected sensing electrodes, adjacent Field Manipulation electrodes at different Voltages, and sets of Shim electrodes at different Voltages, embedded in the lateral wall. A representative result is shown in FIG (VI-3). The velocity contours for Flow Behaviour indices $n=1$ and $n=6$ are plotted in addition to field lines, indicating that field lines have been adjusted to encompass a reasonably large fraction of the velocity contours; FIG (VI-4) shows the overlap of Upstream and Downstream sensors, set at

(")For a strictly Cartesian geometry and applied Potential, Dielectric discontinuity has no effect on field shape; for any other electrode and potential scheme, field lines will cross the discontinuity and suffer refraction. 
$90 \mathrm{deg}$ from each other. Duplicating the CCF calculation for the two pairs of sensor plates that define up/down and left/right overlapping regions, most of the velocity contour can be effectively covered.

A similar scheme can be developed for a duct of circular cross section, where velocity contours are circles while the calculation of equipotentials and Field Lines becomes more difficult; coverage of velocity contours is expected to be less favorable. This calculation is planned for the future. The field-adjustment scheme has some flexibility that may be exploited to provide additional information: by varying the potential in a manner prescribed by calculation, different parts of the velocity spectrum can be acquired and cor-

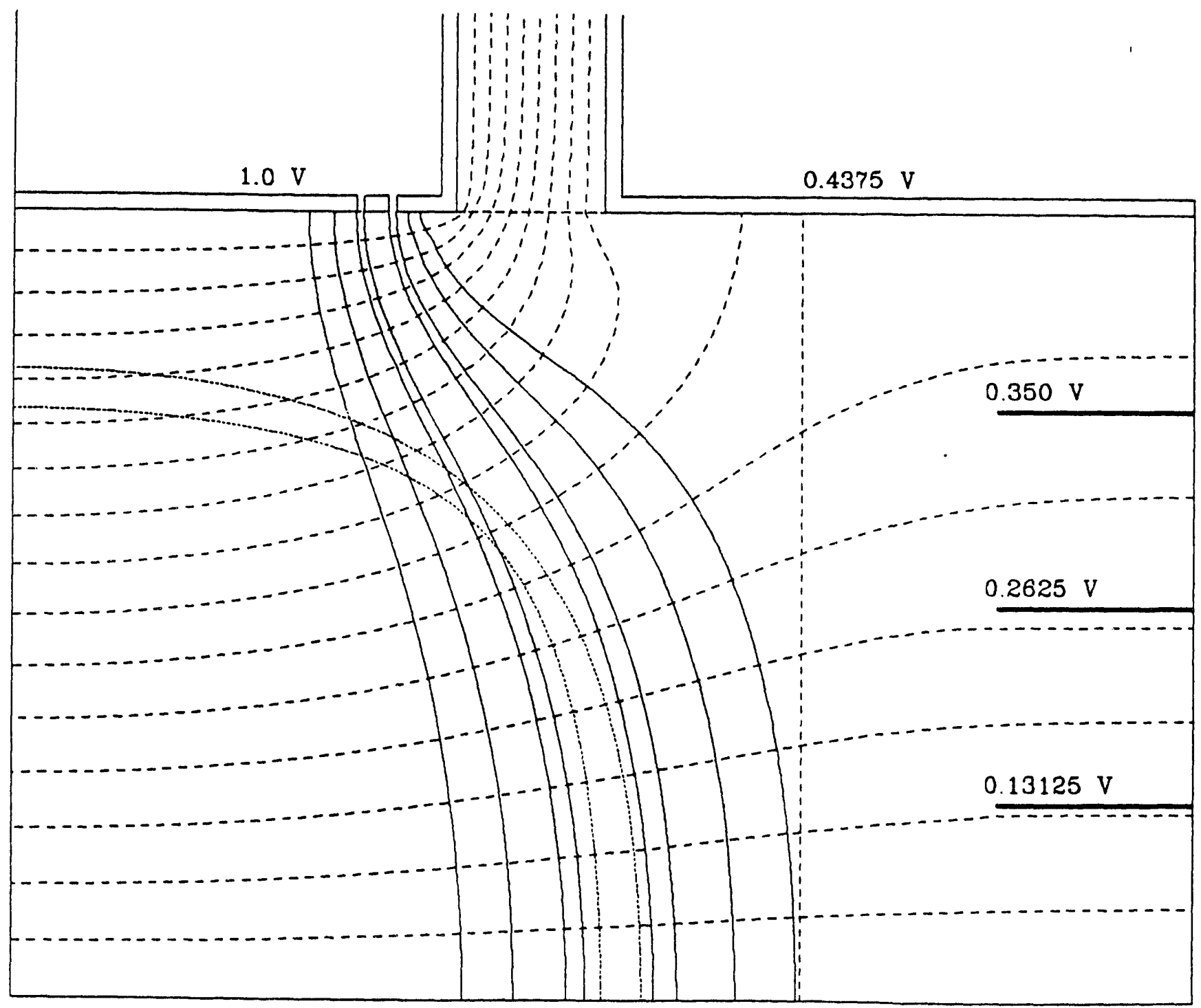

FIG(VI-3) Field-Adjustment Scheme Equipotentials and Field Lines 


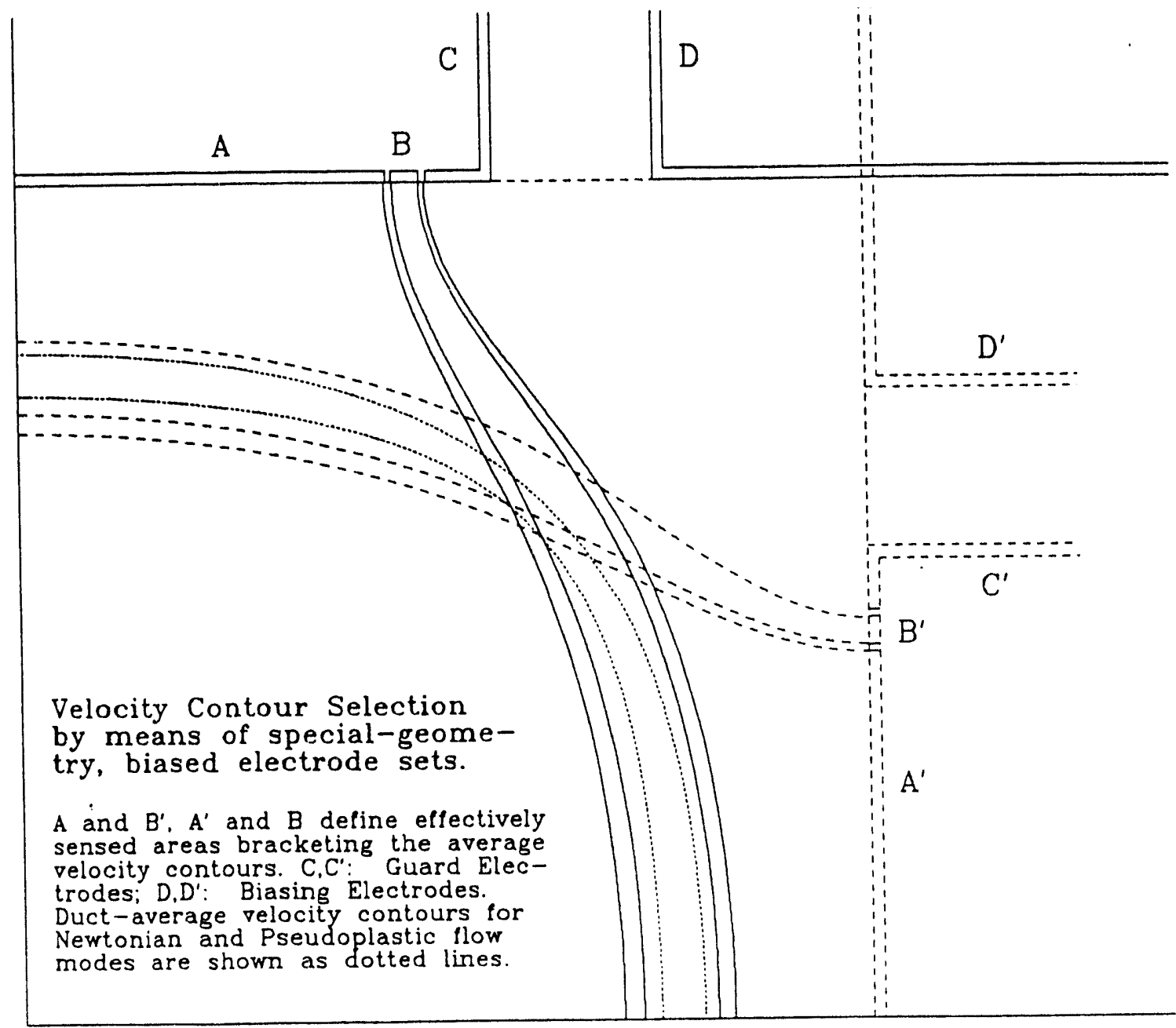

FIG(VI-4): Overlap of Field-Line-Selected Regions and Velocity Contours

related with the Flow Behaviour Index. If additional calculations support this promising scheme, experimental confirmation is called for. A simple test loop, fabricated from plastic and using Methyl Cellulose or a similar viscosi-ty enhancing Chemical, can provide this at low cost.

The input circuit for the Contour-Select scheme must drive symmetrically with respect to the duct center. A possible configuration is shown in FIG (VI5). Output $\mathbf{A}$ of the Upstream sensor is paired with Output B of the Downstream sensor, and vice versa. In other respects, the circuit is self-evident.

In closing, a possible problem that affects all CCF velocity measurements for extremely dense, fine-consist slurries should not go unmentioned: as the medium becomes more uniform, say of the consistency of grease, the size and frequency of inhomogeneities may decline to the point where they will no longer yield a readily distinguishable CCF. This suggests a practical investigation of this possible problem, using the exact medium of a feedline where a CCF velocity measurement is contemplated, before proceeding further. 


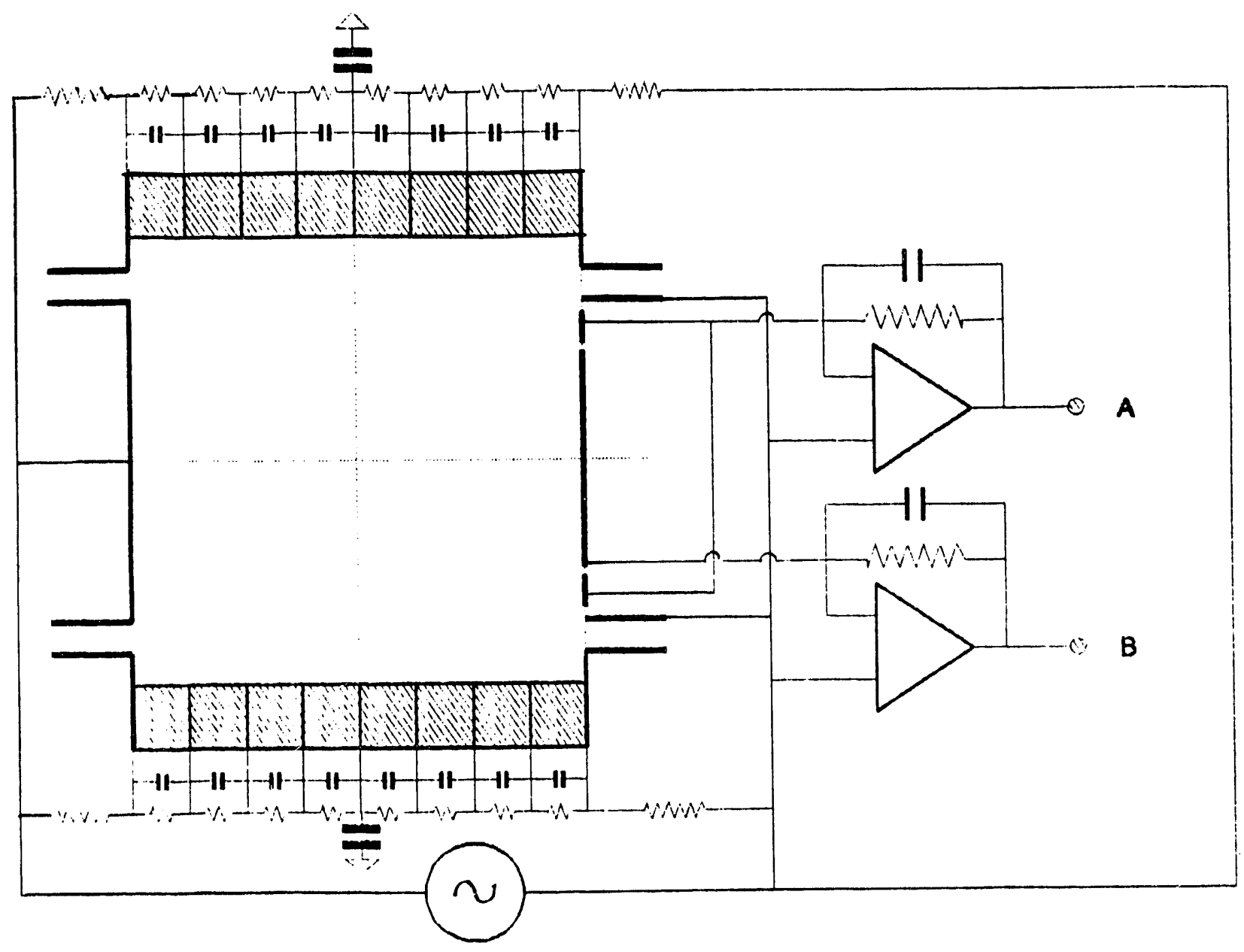

FIG(VI-5): Driving Circuit and Input Amplifiers, Contour-Select Scheme 


\section{SYSTEM DESIGN}

\section{(A) Review of Component Performance}

The preceding detailed description of Flow meter components ustablistred certain performance characteristics that must be taken into account. when rombining Densitometer, Velocity meter and Electronics to provide an oplimized System. In addition to performance, cost must be considered here. As some of the design features that have the best performance (or, in the absence of tests so far, the best potential) are also amongst the more expensive (here again, a realistic estimate is difficult at this time), mix-and-match combinations of conventional, relatively inexpensive devices and the less rostly of the EM sensing schemes reviewed in the preceding are in order for sconarios; that can accommodate such combinations, while applications that must have the best in performance should consider plant efficiency and energy savings that might of fet higher instrument cost.

Reviewing, first, the Densitometry task, TABIL (VII-1) siums; up the? relative performances of the two non-intrusive, on-line schemes whose delailed characteristics are described in the preceding.

TABLE (VI 1-1): PERFORMANCE CHARACTERISTICS, DENSITOMETRY

\begin{tabular}{|c|c|c|c|}
\hline SCHEME & ACCURACY & ADVANTAGES & ORAWBACKS \\
\hline $\begin{array}{c}\text { Gamma Trians- } \\
\text { mission }\end{array}$ & $\begin{array}{c}\text { best }(51 \%) \\
\text { abs. reading. }\end{array}$ & $\begin{array}{l}\text { no calibration: } \\
\text { lab \& book info }\end{array}$ & $\begin{array}{l}\text { fragile } \\
\operatorname{cosily}\end{array}$ \\
\hline $\begin{array}{l}\text { FM fld sens. } \\
\text { (r:HRC I)tu: }\end{array}$ & $\begin{array}{l}\text { good excopt } \\
\text { for lols } \operatorname{error}\end{array}$ & $\begin{array}{l}\text { rugged, controms } \\
\text { los rire. ofuct }\end{array}$ & 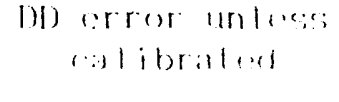 \\
\hline $\begin{array}{l}\text { FM fld inens; } \\
\text { (SoluALE: I). }\end{array}$ & $\begin{array}{l}(: \times(:) \mid \operatorname{lan} t \\
(\because 1 /), \text { abs }\end{array}$ & $\begin{array}{l}\text { no (alliforil inn; } \\
\text { lats \& book infes }\end{array}$ & 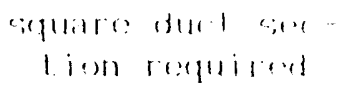 \\
\hline
\end{tabular}

Gamma Transmission Densitometry (n.b., using the type of exuipment described here), clearly the most accurate amongst available non-intrusive drnsitometry schemes, is not really suited for permanent installation in an industrial plant, but certainly could be deployed on a temporary basis. A feed Line site that has been set up so that a gamma device can be readily installed in initial runs and, occasionally, in routine operation could facilitate verification of the EM-sensing, on-line densitometer, provided that the fredstock does not vary considerably from week to week. Alternatively, a liamma densilometer and duplicate EM-sensing device could be installed in a Test. loop. Throse schemes describe an intermediate system, costlier than an EM-sensing scheme that relies entirely on calibration against conventional means and thus a good choice for scenarios where those means are unworkable.

Performance rharacteristics of Velocity (Flow) Meters lhat werr rovicwed in Chapter VI are summarjzod in TABIE (VII-?). As nolod in lhis liablo, mom al

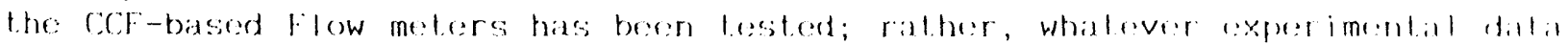

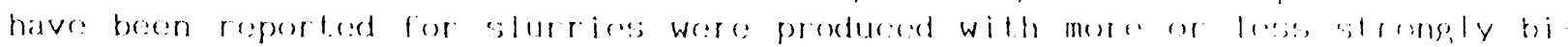

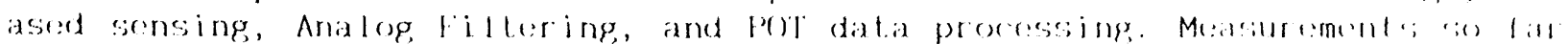
for laminar flow have been force-fitted onto "lincar" performemes plots logether with data measured in turbulent flow, showing, a yero-oflesol that in 
fact indicates a large percentage error (not obvious in this type of plot.). Thus, laminar flow measurements must be considered to have failed altogether. Little is known about Magmeter performance in slurries. As noted in Chapter VI, accuracy-enhanring schemes that improve Magmeler performance for cloan media cannot lalerate slurries and thus must be abandoned. It is also nol clear to what extent dense $160 \%$ by volumej coal slurries at high temperalures reduce the overall conductivity, hence, the EMF that is read out. Coriolis force Flow meters likewise have not been tested with hot, high-pressure media; for any other slurry service, this type of Flow meter has been well-received by the Chemical processing industry.

TABLE(VII-2)： PERFORMANCE CHARACTERISTICS, VELOCITY MEASUREMENT

(A) EM SENSING/CCF

\begin{tabular}{|c|c|c|c|}
\hline DUCT/FLOW MODE & ACCURACY & ADVANTAGES & DRAWBACKS \\
\hline Cir.Det./Turb. & $\begin{array}{l}\cong 1-2 \% \text { possible } \\
\text { w. Momts. Rat.io } \\
\text { processing }\end{array}$ & $\begin{array}{l}\text { conventionial de- } \\
\text { sign; rel. inex- } \\
\text { pensive, robust }\end{array}$ & $\begin{array}{l}\text { untested; existing expl. } \\
\text { data inacrurate (biasml } \\
\text { sensing. por datia prom. }\end{array}$ \\
\hline Sq. Det./Turb & $\begin{array}{l}\text { not lested; exp } \\
\cong 1 \%: 100 \% \text { sen- } \\
\text { sing uniformity }\end{array}$ & $\begin{array}{l}\text { design inherently } \\
\text { more accurate than } \\
\text { rirc. dot. design }\end{array}$ & $\begin{array}{l}\text { expect fabricn. of sq. } \\
\text { duct section rol. mor: } \\
\text { costly, also untested. }\end{array}$ \\
\hline Cir.Dct./Lam. & $\begin{array}{c}\text { ? spec. electr. } \\
\text { gcom. not yet. } \\
\text { designed }\end{array}$ & $\begin{array}{l}\text { to offer better } \\
\text { accuracy than } \\
\text { circ. design can }\end{array}$ & $\begin{array}{l}\text { not known; untested; } \\
\text { cost of design, labrica- } \\
\text { tion higher than cirr.? }\end{array}$ \\
\hline sif. Dr:t. & $\begin{array}{l}\cong 1-2 \% \text { est. } w \\
\text { opt. imum contour } \\
\text { selort design }\end{array}$ & $\begin{array}{l}\text { the only workable } \\
\text { cot geo. designed } \\
\text { for laminar llow. }\end{array}$ & $\begin{array}{l}\text { untested; square du: is } \\
\text { required \& may bo wepen- } \\
\text { sive. }\end{array}$ \\
\hline
\end{tabular}

(B) MACIMI:TH:R

\begin{tabular}{|c|c|c|c|}
\hline Cir.Dct. /all & $\begin{array}{c}\text { - } 1-2 \% \text { for dense } \\
\text { slurry/lam; } \\
\cong 1 \%, \text { turb? }\end{array}$ & $\begin{array}{c}\text { conforms to } \\
\text { circ. duct; } \\
\text { widely used }\end{array}$ & $\begin{array}{c}\text { needs conducting medium; } \\
\text { Has not workod woll } \\
\text { in slurries, so lar }\end{array}$ \\
\hline
\end{tabular}

(C) CORIOLIS' FORCE FLOWMETER

\begin{tabular}{|c|c|c|c|}
\hline Cir.Dct./all & $\cong 1 \%$ turbult. ; & $\begin{array}{c}\text { same as for } \\
\text { MAGMETER. }\end{array}$ & $\begin{array}{c}\text { introduces flow lmped- } \\
\text { ance; not available } \\
\text { for }>1.5 \text { in dia. duct. }\end{array}$ \\
\hline
\end{tabular}

As concerns pipeline applications, there is some hope that Cross-Correlation can be made accurate enough for turbulent flow lencountered in pipeline conveyors). As CCF is a computation-intensive scheme, one can confidently oxpect a progressively declining cost of the computation equipment and sofl.ware component; CCF also seems a cost-effective solution for a large duct. diamoter Magmeters of more than $1-\mathrm{ft}$ diameter are rare and expensive; (oriolis' flow Melers for more than 1.5-in ducts have not bexen successtull).

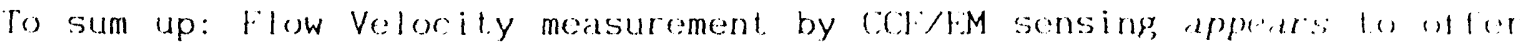
accurate performance for either laminar or turbulent flow but must be considered as untested; Magmeter performance for dense slurries needs some improve- 
ment. (that may well be forthcoming from ongoing laboratory work of instrument. firms); Coriolis' force l-M likewise must still be demonstrated for feedlines.

\section{(B) Cornbined Calculations}

A computer of PC-386(486) type, supported by a dedicated corrolation computer board of type described in Chapter VI, has adequate speed and memory for the complatations involved in the acquisition (via plug-in Interface Board), processing, display and storage of the information connected with the solids feedrate. Such computers are available from a number of vendors with "industrial" abuse-resistant boxes; plug-in boards of many types are likewiso aviailable at low cost. The necessary soltware package, written in large part in Assembler, would have to be developed; other programs could be written in lonTRAN or C.

As discussed in Chapter IV, I:M density sensor samples must be: read it at rate that minimizes fluctuation during sampling(*). At intervals that corrospond to several thousand such samples, the calculations indicated in lhapler IV are performed; at an even slower rate, Track-Switching is implementer; all under computer control. The last. step in density processing, a siucressivivapproximation solution of the cubic reversed-Looyenga equation, yields the solid volume fraction $\phi$.

At the same time, samples intended for the CCF velocity measurement are: read, averaged and trimmed to bipolar - 1 byto format, then transterred lo the: speed-board which proceeds to execute the operations described in litaptor VI.

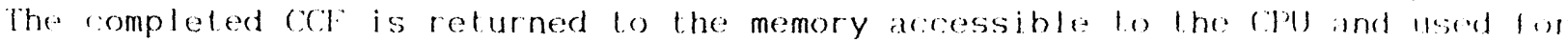

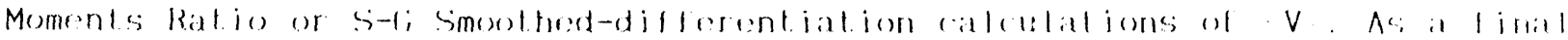

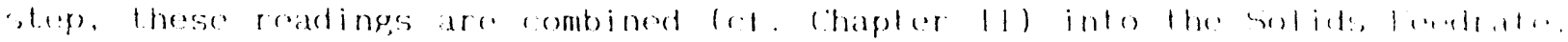

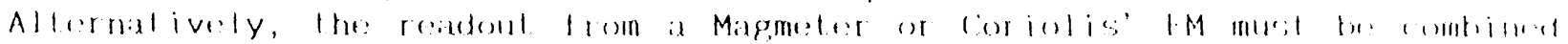

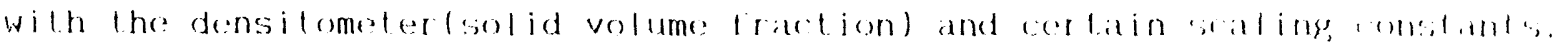

Another computer and program would be needed for a lossl loxp anm wllm. laboratory devices, discussed in Chapter $V$, for calibration/verifiration ol density readout and for ineasurement of intrinsic solids parameters (dieleatric. constant and specific gravity) that are poorly known for coal, yet needed in medium density measurement.

All computations require the input of component-specifir informition le.g., a set of solid component weight fractions; density and formula of the fluid, duct and sensor device dimensions). This, and other intormation inpul. can be implemented by an interactive program of "menu" lype.

Storage, integration, Display and Transfer to another computer for Process Control are typical options that can be implemented in arbitrary fashion, as preferred by plant personnel or management. Modular programs can be provided to facilitate any customized version.

At some future time, a program that corrects for dielectrir discontinuity may be available and can be stored on disk, ready for appliarlion in an initial setup and later contirmalion.

(")ll the Iluctuation error turns out to be small or negligible, lhe lasalsampling scheme can be replaced by sampling at readout intervals. 


\section{SUMMARY AND CONCLUSION}

\section{(A) Summary}

The on-line measurement of the solids feedrate in Industrial dense-slurry conveyors, the subject of this report, is needed by the Chemical Processing Industry; yet, it cannot be provided by means that are commercially available, or described in the literature. Although this literature is extensive and covers devices based on a large variety of principles, one finds that the extremely hostile medium, together with the reliability, accuracy and readout delay that would make it useful for process control, define specifications which conventional devices and systems cannot meet. The first and second Chapters of this report review and comment on those difficult specifications, summarized, for reference, in TABLE (VIII-1).

TABLE (VIII-1): CONDITIONAL SPECIFICATIONS FOR FLOW MEASUREMENT

IN DENSE, ABRASIVE LIQUID/SOLID MEDIA

\begin{tabular}{|c|c|c|c|c|c|c|c|}
\hline $\begin{array}{l}\text { SOLID } \\
\text { FRACT }\end{array}$ & $\begin{array}{l}\text { TEMP } \\
\text { deg C }\end{array}$ & $\begin{array}{c}\text { PRESS } \\
\text { atm. }\end{array}$ & \begin{tabular}{|l|} 
TYPICAL \\
FLOW MODE
\end{tabular} & $\begin{array}{l}\text { TYPICAL INDUSTR I AL } \\
\text { APPL ICATION }\end{array}$ & $\begin{array}{l}\text { TYP DUCT } \\
\text { DI AMETER }\end{array}$ & $\begin{array}{l}\text { MAX \% } \\
\text { ERROR }\end{array}$ & $\begin{array}{l}\text { MAX } \\
\text { DELAY }\end{array}$ \\
\hline $\begin{array}{r}0.5 \\
0.6\end{array}$ & $\begin{array}{c}\mathrm{amb}- \\
300^{\circ}\end{array}$ & $\begin{array}{ll}1- \\
50\end{array}$ & $\begin{array}{l}\text { Lam. /Non - } \\
\text { Newtonian }\end{array}$ & $\begin{array}{l}\text { Chem. Reactor, Fluidized } \\
\text { Bed furnace sol. feedline }\end{array}$ & $\begin{array}{r}0.5 \text { to } \\
2 \text { in }\end{array}$ & $\begin{array}{l}\cong 0.5 \\
-1 \%\end{array}$ & $\begin{array}{l}\cong 1 \\
\sec \end{array}$ \\
\hline $\begin{array}{r}0.3- \\
0.4\end{array} \mid$ & amb. & 1 & $\begin{array}{l}\text { Turbulent- } \\
\text { Transition }\end{array}$ & $\begin{array}{l}\text { In-plant, Over land (Pipe- } \\
\text { line) solids conveyance }\end{array}$ & $\begin{array}{r}0.5 \text { to } \\
48 \text { in }\end{array}$ & $\begin{array}{l}\cong 1.0 \\
-2 \%\end{array}$ & $\begin{array}{l}2-4 \\
\sec \end{array}$ \\
\hline
\end{tabular}

From the set of Conditional Specifications, a corresponding set of Instrument Specifications can be inferred, summarized in TABLE (VIII-2):

TABLE(VII I-2): INSTRUMENT SPECIFICATIONS

\begin{tabular}{|c|c|}
\hline$\#$ & SPECIFICATION \\
\hline 1 & - Two independent measurements, TASK (A) \& TASK (B) \\
\hline $\begin{array}{l}2 \\
3\end{array}$ & $\begin{array}{l}\text { Instruments for both tasks must have: } \\
\text { - Non-Intrusive Construction } \\
\text { - Absolute Readout (dispensing with Calibration need) }\end{array}$ \\
\hline $\mid \begin{array}{l}4 \\
5 \\
6\end{array}$ & $\begin{array}{l}\text { Specification } 3 \text { can be satisfied through: } \\
\text { - Uniform Sensing within, No Sensing Outside Duct } \\
\text { - a well-developed Theoretical Framework } \\
\text { - Error Correction Capability }\end{array}$ \\
\hline
\end{tabular}

Requirement \# 1, dual, independent measurements, logically follows from the rule: Number of Modes $=$ Number of independent measurements required for sensing individual phase discharge. As Process Control usually requires the rate of flow of the solid component rather than the overall flow rate, two independently varying parameters must be measured; these measurements, designated here as TASK (A) and TASK (B), can be chosen from among Phase Fraction, 
Medium Velocity and Total Mass Flowrate (all of which are measurable). This dual measurement requirement, seldom emphasized though generally accepted, has the effect if raising the cost of the complete System, consisting of two independent measurement channels and, in addition, an on-line computer. Though the cost of the latter is decreasing from year to year, software can add significantly to the initial cost: needed programs include reading individual channels, correcting and converting the readings, combining them to the solids Feedrate (and perhaps other useful, but not crucial, derived variables), calculating probable error and passing the information on the a Process Controller; a more mundane, inexpensive task is that of displaying (in any desired format), integrating and, finally, storing the information. Requirements $2-4$ shown in TABLE(VIII-2) are now briefly discussed:

Requirement \#2, Non-Intrusive Construction is mandated by the highly abrasive-erosive nature of dense-slurry media, which, as universally agreed, tend to destroy whatever protrudes into the stream; to this, one may add the danger of plugging threatened by such protruding hardware and even by local duct constrictions.

Requirement \# 3, Absolute Readout Capability, has not been emphasized but logically follows as one becomes aware how severely the medium, possibly heated and at high pressure, inhibits conventional calibration and how onerous repeated of $f-l i n e$ calibration becomes when the solid feedstock is highly variable (such as, e.g., coal). If not absolute-reading in the strictest sense, a reasonably usable instrument would be one that can deliver a direct readout after an initial calibration, possibly repeated at, say, one-year intervals. Conversion of the device readout to the desired variable could involve information published in the form of Tables, information such as fluid and solid Chemical composition, intrinsic density and dielectric constant, together with temperature-variability of the latter two parameters. To the extent to which this information is difficult to obtain (which is notoriously the case for coal), laboratory determinations would be resorted to. Now, as it happens, all commercially available on-line flow meters that can deliver an absolute readout are intrusive; clamp-on and duct surface-mounted devices all seemingly require calibration. The question thus arises whether, and under what conditions, these available schemes can be made absolute-reading in the sense specified above. This question can be usefully recast in the form of Necessary Conditions, Requirements \#4-6; together, they form a Sufficient set.

Requirement \#4, Uniform sensing within, No sensing without the duct, is a logical consequence of the Fluid Mechanics of slurries, which therefore is briefly reviewed in Chapter III. The salient point here is the local variation (across the duct) of both the solid fraction and, especially, the medium velocity. To determine the potential of some specific measurement device to provide such uniform coverage evidently calls for

Requirement \#5, a well-developed theoretical framework that allows the quantitative calculation of the local sensing efficiency for the device in question. Aside from non-uniform coverage of the duct per se, other errors, arising from various causes, may be present in the readout; if so, then one is led to postulate

Requirement \#6, error correction capability. The ready availability of an on-line computer makes fairly sophisticated error-correction feasible, provided that available knowledge concerning the sensing device allows one to develop the necessary sof tware. 
Having thus developed a set of Instrument Specifications, it is expedient to begin by examining the capability of existing non-intrusive schemes and devices with regard to meeting the specifications. This examination, dealing first with on-line densitometers and then, with velocity meters, and reviewing the principles on which devices are based in some detail, leads to certain specific recommendations. These reviews and conclusions form the bulk of this report. In the course of examining existing measurement schemes, some of these, non-intrusive and highly effective in more benign situations, turn out to be inherently unable to sense the duct uniformly; amongst others, Acoustic (Supersonic) Devices fall into that category. Acoustic sensing also lacks the support of a sound theoretical framework (comparable, say, to the theory of Gamma Transmission) and ipso facto has no Error-Correction Capability. This inevitably suggests that, for dense-slurry flow measurement, acoustic devices are less promising than other available schemes. This judgment, it must be emphasized, is limited to Dense Slurry Conveyors and other situations that militate against conventional calibration. Acoustic sensors in Diagonal Path or Doppler systems are widely and successfully applied in turbulent flow of pure fluids and dilute slurries, where calibration is readily feasible.

Devices that best meet the set of Specifications imposed by the medium are listed in Table (VIII-3), with rubrics for Conducting and Dielectric Slurries and for the two selected tasks of Solid Volume Fraction and Flow Velocity measurement.

TABLE (VIII-3): TASKS (A) AND (B): ACCEPTABLE DEVICES

\begin{tabular}{||c||c|c||}
\hline TASKS & (A): SOLID VOLUME FRACTION & (B): FLOW VELOCITY \\
$\begin{array}{c}\text { Conducting } \\
\text { Slurry }\end{array}$ & $\begin{array}{c}\text { E.M. Field Interrogation } \\
\text { Gamma Beam Transmission }\end{array}$ & $\begin{array}{c}\text { Magmeter } \\
\text { Coriolis' FM } \\
\text { (using EM sensing) }\end{array}$ \\
\hline $\begin{array}{c}\text { Cielectric } \\
\text { Slurry }\end{array}$ & Same as above & $\begin{array}{c}\text { Coriolis' FM } \\
\text { (using EM sensing) }\end{array}$ \\
\hline
\end{tabular}

The instruments listed in this Table are discussed in detail in this report; their pertinent characteristics are summarized below.

(1) SOLID VOLUME FRACTION - Gamma Transmission: Commercially available instruments for on-line use are designed to trade of $f$ accuracy for resistance to damage. Lacking energy selectivity and adequate beam collimation, these instruments respond to scattered and secondary radiation, rendering their readout site-sensitive and unresponsive to theoretical interpretation. In contrast, equipment routinely used in laboratories (but less damage-resistant) is energy-selective, configured in Good Geometry and equipped with high countrate electronics. With these features, theoretical interpretation becomes feasible, yielding an absolute determination of the Phase Fraction with certain additional information that can be obtained by of $f-l$ ine measurement. As the transmitted fraction is largely unaffected by phase distribution (stratification), beam coverage of the entire duct is not important. Gamma Transmission 
has an inherent 3-phase (Solid/Liquid/Gas) capability that is unique and (like the 2-phase scheme) can be rendered highly accurate and self-calibrating. Performance characteristics of Commercial and Energy-Selective Gamma Transmission Systems are compared in TABLE (VIII-4).

TABLE (VI I I-4): GAMMA TRANSMISSION DENSITOMETRY

\begin{tabular}{|c|c|c|}
\hline CONCERN & RESPONSE: COMMERCIAL & RESPONSE: ENERGY-SELECTIVE \\
\hline $\begin{array}{l}\text { Scattered rad. } \\
\text { Error }\end{array}$ & \begin{tabular}{|c||} 
duct wall \& medium-scatt. $\&$ \\
secondary radiation registers
\end{tabular} & $\begin{array}{l}\text { Energy-Sel. \& collimation eli- } \\
\text { minate scat. rad. registration }\end{array}$ \\
\hline $\begin{array}{l}\text { Fluctuating } \\
\text { Phase fraction } \\
\text { Error }\end{array}$ & Can' $t$ be eliminated & $\begin{array}{l}\text { Elimination by Short } \\
\text { Interval Acquisition } \\
\text { Strategy }\end{array}$ \\
\hline Overall Error & $>5 \%$ & $<0.5 \%$ \\
\hline $\begin{array}{l}\text { Electronics } \\
\text { Concerns }\end{array}$ & $\begin{array}{l}\text { Current-read Analog Electron-| } \\
\text { ics can't be Stabilized; need } \\
\text { additl. Digitization Stage. }\end{array}$ & $\begin{array}{l}\text { Pulse-read Analog Electronics } \\
\text { can be Stabilized (light puls.) } \\
\text { Digitization provided by Scaler }\end{array}$ \\
\hline Calibration & $\begin{array}{l}\text { Indispensable; must be done } \\
\text { every time the Chem. Composi- } \\
\text { tion of the medium changes }\end{array}$ & $\begin{array}{l}\text { Replaced by Calculation using } \\
\text { intrins. density, Chem. Comp. } \\
\text { (for coal, H-fraction suffices) }\end{array}$ \\
\hline $\begin{array}{l}\text { Other Con- } \\
\text { siderations }\end{array}$ & $\begin{array}{l}\text { Relatively inexpensive } \\
\text { Damage Resistant } \\
\text { Industry-Accepted }\end{array}$ & $\begin{array}{c}\text { Relatively costly } \\
\text { Delicate } \\
\text { Novel in Indust. plant install. }\end{array}$ \\
\hline
\end{tabular}

(2) SOLID VOLUME FRACTION - E.M. Field Sensing: Of a variety of existing E.M. Field (or, "capacitive") devices, none meets the requirement of unbiased sensing: paradoxically, many electrode geometries that are described in reports and patents are found, by solving the Poisson Equation and plotting the equipotentials, to sense in an especially strongly biased manner. For a duct. of circular cross section, the poor sensing uniformity of the widely used surface-conforming "Wrapped-Plate" scheme can be significantly improved by the Guard \& shim geometry of the ANL Mk 2 device. However, uniformity and other needed performance characteristics become good enough to obtain acceptable readout accuracy only when a square duct section is provided. EM Sensing requirements are summarized in TABLE (VIII-5).

TABLE (VIII-5): E.M.FIELD SENSING REQUIREMENTS

\begin{tabular}{|l|l|}
\hline Device Geometry Design Goals: & Uniform Sensg. Inside, No Sensg. Outside Duct \\
\hline Requirement (a) : & Reference Measurement, $e_{f}$ \\
\hline Requirement (b): & Equation connecting $e_{o}, e_{f}$ and $k_{m}, k_{f}$ \\
Requirement (c) : & Equation connecting $k_{m}, k_{f}, k_{s}$ and $\phi_{s}$ \\
\hline
\end{tabular}

The Equations called for in this Table exist and can be incorporated into an on-line computation routine. To convert the densitometer voltage signal to the bulk dielectric constant, one needs a reference measurement (duct filled with pure fluid); with that, EM Field sensing can dispense with calibration, whether for densitometry or for cross-correlation velocity measurement. TABLE 
(VI I -6) summa cular or squar

TABLE (VI I I -6

\begin{tabular}{|c|}
\hline CONCERN \\
\hline $\begin{array}{c}\text { Sensing Unif, } \\
\text { Error }\end{array}$ \\
\hline $\begin{array}{l}\text { Dielectric Di: } \\
\text { nuity Error }\end{array}$ \\
\hline $\begin{array}{c}\text { Cross-Corr. V, } \\
\text { Measuremer } \\
\text { Turbulent/Tra! } \\
\text { Flow mode }\end{array}$ \\
$\begin{array}{c}\text { Cross-Corr. V. } \\
\text { Measuremen } \\
\text { Laminar-Newto } \\
\text { Pseudoplastic }\end{array}$ \\
\hline
\end{tabular}

The vario the preceding by the square text describes cubic equation

For the $b$ be avoided and neglected in $t$ related to a 1 As described i forward means ject to interf quency is high ty meters, vir zes EM sensing characteristics for both tasks, in either cirluct configuration.

\section{7}

E.M. FIELD SENSING CHARACTERISTICS, CIRCULAR \& SQUARE DUCTS

\begin{tabular}{|c|c|c|}
\hline & CIRCULAR DUCT CROSS SECTION & SQUARE DUCT \\
\hline nity & fair at best (Wrapped-Pl w.Guard) & near-perfect \\
\hline i um & $\begin{array}{l}\text { varies with electrode geometry; } \\
\text { Some error unavoidable }\end{array}$ & $\begin{array}{l}\text { error correctable } \\
\text { by vert/horiz set }\end{array}$ \\
\hline ti- & $\begin{array}{l}\text { error unavoidable; correction is } \\
\text { feasible but difficult }\end{array}$ & $\begin{array}{l}\text { No Diel. Disconti- } \\
\text { nuity Error }\end{array}$ \\
\hline city & $\begin{array}{l}\text { Conventional POT inevitably produ- } \\
\text { ces large error. Moments Ratio } \\
\text { eliminates that error, leaves only } \\
\text { errors described above. }\end{array}$ & $\begin{array}{l}\text { Convent. POT again } \\
\text { yields large error; } \\
\text { with Moments Ratio, } \\
\text { highly accurate. }\end{array}$ \\
\hline city & $\begin{array}{l}\text { Both Conventional \& Moments Ratio } \\
\text { Fail; calculations suggest Contour } \\
\text { Selection scheme with est. error } \\
\text { of } \cong 2 \%\end{array}$ & $\begin{array}{l}\text { Both POT, Mom. Ratio } \\
\text { Fail; calculations } \\
\text { show Contour Selec- } \\
\text { tion is Feasible }\end{array}$ \\
\hline
\end{tabular}

errors listed in the first column (and discussed at length in kt), such as the "dielectric discontinuity" error, are avoided zometry, another reason for adopting that configuration. The he design of a circular-to-square transition piece, based on a

$t$ achievable accuracy, a number of minor causes of error must rectified. A Phase fluctuation error in the density readout, literature but present whenever any readout is non-linearly ctuating variable, affects all on-line densitometry schemes. Chapter IV, the use of an on-line computer offers a straighteliminating this type of error. Conducting slurries are subence by resistive signal components unless the driving frenough to make them negligible (E.M. sensors become conductivially impossible to calibrate, at low frequencies).
(3) VELOC stream and Do! going back to were primitive the former im: quence of pris and well-meant too inaccurate curate is att RPI and PNA, , accurate means finds that $P N$. reported with
$Y$ by CROSS-CORRELATION of EM Sensor Signals from identical Upstream sensors has had a perhaps surprisingly long history, e late forties. During most of that time, means of calculation hence, procedures for data evaluation equally primitive; when sved, the latter were, unfortunately, retained. As a conseive data processing, use of strongly biased sensor geometry ut clumsy filtering. Cross-Correlation has thus far been $f . \mathrm{r}$ or Industrial application. That the method is potentially acied by two generically similar velocity measurement schemes, t, while unsuited to on-line deployment, are amongst the most if velocity measurement in Test loops. Pursuing this lead, one (Pulsed Neutron Activation) has had a similar history: first radequate data processing, it became highly accurate only with 
the development of Moments Ratio data processing, by Fluid Mechanics modeling. As shown in this report, the same process, when applied to CCF, yields a similar Moments Ratio for turbulent flow. The necessary calculations are nowadays readily performed, at more than adequate speed, by PC-type, inexpensive computation equipment, incorporating if necessary a "fast" hardware plug-in board.

The modeling treatment reveals, however, that Cross-Correlation transit timing must fail in laminar flow (as indeed it has, in a number of tests). To cope with laminar flow, the prevailing mode in slurry feedlines, a Contour Selection scheme is proposed in this report. As the flow mode becomes more and more Pseudoplastic (i.e., with declining Metzner Flow Behaviour Index), the general flow profile changes considerably, yet the "Specific" contour (where the local velocity just equals the duct-average) remains almost unchanged. A set of calculations indicate that the specific contour can be bracketed by certain combinations of plate geometries and voltages, in a manner that. selects only those particles/clusters that move close to the selected contour.

\section{(B) Conclusion}

While there is wide agreement on the necessity of providing non-intrusive devices for on-line flow measurement in dense slurries, the additional requirement of an absolute readout capability, suggested in this report, is perhaps somewhat unusual. This requirement seems to follow from the technical difficulty of calibration on one hand, and the need for frequent calibrations when a substance like coal is conveyed, on the other hand.

The first task this report sets itself is to select non-intrusive sensing schemes that appear to have the potential for absolute reading: Gamma Transmission and E.M. Field sensing Densitometers and E.M. Field sensing, CrossCorrelation velocity meters.

Each of these sensor types is already available and used in on-line Flow measurement; however, detailed investigation reveals performance deficiencies that call for correction by means of specific design of the sensing geometry and channel electronics. For Gamma transmission, current practice in designing systems used for research rather than Industrial Plant deployment can be taken advantage of. That energy-selection and collimation are needed to validate the exponential attenuation law can be assumed well-known; likewise, relations between cross sections and atomic number are hardly novel. However, it may not have been widely appreciated that for a low- $Z$ medium especially rich in eveneven atomic species, such as coal, a medium composition-independent formula based on $Z / A=0.5$ and $\sigma(Z)=Z \times \sigma(H)$ comes fairly close to the true at tenuaticn coefficient. Thus, a correction that needs only the hydrogen content of solid and fluid improves the result to < $1 \%$; a $\mathrm{final}$ correction, based on a few more major components, removes even that small error. Calculations and measurements for both high-ash and low-ash coal support these observations. These useful facts can be exploited in an on-line densitometer or in a benchtop laboratory measuremen: of solids (coal) intrinsic density; the former can be used in a miniature test-loop for calibration of cther on-line densitometers, the latter supports the former isince density is not reported as part of the ASTM informationl. 
Turning to the design of E.M. Field or "capacitive" sensing systems for on-line densitometry, a variety of device geometries has been described or is commercially available, yet, none have adequate sensing uniformity. This is readily revealed by calculations that, for some reason, don't appear to have been made in connection with these designs. The poor quality of sensing uniformity thus seemed to call for some sort of drastic improvement in geometry. Two solutions are proposed in this report: first, a modified "Wrapped-Plate" geometry for circular ducts, secondly, a transition to a square duct shape (whose sensing uniformity as well as duct coverage is clearly unbeatable). A square duct shape further offers a remedy for stratified-flow error and of fers an indication of the degree of stratification, in horizontal ducts.

Just like Gamma transmission, an absolute E.M. sensing readout requires a theoretical framework that allows the conversion of E.M. readout signals to the phase fraction. Since E.M. sensing has conventionally relied on calibration, that framework is not mentioned in the instrumentation literature, but can be found elsewhere. The case thus had to be made for basing the calculated conversion of signals on little-known theoretical formulae. In turn, this called for reviewing what is known about binary dielectric mixtures, thereupon applying the mixture formula in model calculations for stratified media.

The effect of phase fluctuations on density measurement seems to have been overlooked for EM sensing (though well-known for Gamma Transmission). The resulting offset error can be eliminated by a "rapid sampling" data acquisition strategy: Another useful result of this exercise in elementary Statistics is a simple Flow/No Flow indicator based on a single sensor channel. E.M. sensors intended for absolute readout are affected by a "dielectric discontinuity" error, connected with the need for a reference measurement, that remains to be effectively dealt with; it is absent in square ducts.

The conventional processing of Cross-Correlation data, based on adherence to outdated procedures originally adopted only because computation equipment allowed no alternative, has handicapped this potentially useful technique of flow measurement. To remedy that situation, modeling, successful in PNA, can be employed, yielding a moments ratio, readily computed from the CCF, for turbulent flow. Laminar flow constitutes a special case; few Flow meters of any design yield accurate velocity readings in that flow mode. A preliminary set. of calculations, reported here, suggests that velocity contour selection through special plate geometries and applied voltages could yield velocity indication with an error margin of a few per cent.

None of these general conclusions were apparent in 1978, at the beginning of the Flow Measurement Initiative that has been repeatedly referred to. Rather, it seemed that a large variety of diverse instruments was already available. This led to the conventional approach of subjecting selected devices to series of tests at Industrial and Test Loop sites. As PC-type computers were not yet available, calculations were costly and difficult, hence, generally de-emphasized. Today, calculations have become easy and inexpensive even on a sophisticated level. At the same time, the evident failure of conventionally configured capacitive (as well as acoustic) devices in the two slurry measurement series makes it evident that any further tests can only be inconclusive and/or misleading unlesc preceded by detailed design calculations that allow the fabrication of an effective device. Once such a design is available, then confirmation by measurements, especially in laminar flow and over a wide range of Flow Behaviour indices, is called for. 
Other useful calculations include the extension of the specifics developed in this report to gas-entrained solids flow, increasingly the method of choice for conveying solids within a plant. The velocity measurement algorithm for turbulent flow can be tested inexpensively yet convincingly through MonteCarlo simulation.

Measurement tasks that are needed to support design calculations include a series of coal intrinsic density and dielectric constant measurements, useful not only for on-line densitometry but also in correlating coal characteristics such as reactivity and heat of combustion. Already available nonintrusive Flow velocity meters (such as Magnetic or Coriolis' Force meters) have not been sufficiently tested with dense, pre-heated slurries and may need to be improved to perform adequately in that service; nor has their potential for absolute readout been developed.

In closing, we should like to add that more Fluid Mechanics data on dense slurries are also highly desirable, both for meter development and for design of efficient conveyance systems. For example, lit.te information is available on particle and fluid velocity distributions for various particle sizes and concentrations in dense slurry flow. The RPI (Radioactive Particle Injection) scheme described in Chapter. VI is the only existing means that can provide this information for dense slurries as well as dense gas-entrained solids flow. However, its practical implementation requires equipment and large installations that are available at relatively few laboratories, including ANL. (where promising results have already been obtained in preliminary work).

\section{ACKNOWLEDGEMENTS}

The authors acknowledge the support and encouragement of Drs. L. I.cSige and $E$. Bennett, without which this report would not have been issued, with sincere gratitude. Dr. O'Fallon's patient and understanding leadership in the first phase of the program was likewise appreciated. We are indebted to Dr. Schmalzer, manager of Fossil Energy Programs, for an up-to-date review on the use of slurry feed in coal utilization. Discussions with our colleagues C. Herzenberg, F. Levert, D. Bobis and M. Farahat helped to clarify ideas; for any mistakes in this report, the blame is ours alone. F. Lenkzsus designed a prototype fast board that produced the first. CCF computation at a practicial speed but has now, inevitably, been obsoleted by the rapid pace of progress in Electronics. The late Bill Managan should be credited with being the first to apply Poisson Finite-Difference calculations to E.M. Flectrode design. Calculations in this report were encoded in FORTRAN and exocuted on a 20 MHz DOSS computer with 386 repl and co-processor. The manuser ipt wass written and edited in CHI 1.2; Figures were plotted with CRAPHER and DRAW PFRIFECT. 


\section{LITERATURE CITED}

CHAPTER I

( I-1) var. auth., "HYDROTRANSPORT 1"-"HYDROTRANSPORT -", Proc. of a series of Intnt1. Conf. on the Hydraulic Transport of Solids in Pipes, BHRA-Fluid Eng., Cranfield, Bedford MK 43 OAJ, England, 1974 -

( I-2) var. auth., Proc. Conf. on Instr. \& Contr. for Fossil Energy Processes:

( I-2a) ANL-78-62(CONF-780656), Newport Reach CA;

$(\mathrm{I}-2 \mathrm{~b}) \quad$ ANL-79-62(CONF-790855), Denver CO;

$(\mathrm{I}-2 \mathrm{c}) \quad$ ANL-80-62 (CONF-80602), Virginia Beach VA;

$(\mathrm{I}-2 \mathrm{~d}) \quad$ ANL-81-62(CONF-810607), San Francisco CA;

$(\mathrm{I}-2 \mathrm{e}) \quad$ ANL-82-62(CONF-820612), Houston TX.

( I-3a) Porges, K.G., Cox,S.A., Brewer,W.E. \& Hacker, D.S.: "System Description of the ANL Slurry Loop Testing Facility (SLTF)", ANL/FE-84-20 (1986)

(I-3b) Porges, K.G., Lenkzsus, F.R., Cox. S.A., Kampschoer, C. J., Cohn, C.E., Groh, E.F., Lewandowski, E.M. \& Hacker, D.S.: "A Slurry Test Loop for Flowmeter Evaluation and Calibration", (I-2d) ff.

(I-3c) Porges, K.G., Kampschoer, C.J., Cox, S.A. \& Brewer, W.E.: "Some Recent Slurry Flow Measurements with the ANL-SLTF", (I-2e) ff.

CHAPTER II

(I I-1a) Aude, T.C. Cowper, N.T., Thompson, T.C. \& Wasp,E.J. : "Slurry Piping Systems: Trends, Design Methods, Guidelines", Chem. Eng.78, 74 (1971)

(II-1b)Aude,T.C.\& Pitts, J.D.: "Slurry Pipeline Design", Tech. Conf. on Slurry Transport, Columbus $\mathrm{OH}$, Feb 1976

(II-2) Buckwalter, R. K.: "Instrumentation for Slurry Pipelines", InTech, Jun 1979 , p 65

(II-3) Carleton, A.J. \& Cheng, C-H: "Pipeline Design for Industrial Slurries", Chem. Eng., Apr 1977, p 95

(II-4) Wasp, E.J.: "Comparative Economics of Slurry Lines", Slurry Pipelining Course, Banff, Alberta Mar 1976

(I I-5) Prakash, A. \& Bendale, P.G.: "Design of Slurry Reactor for Indirect Liquefaction Applications", Viking Systems Int. Pittsburgh, PA 1991

(II-6) Report to the APS by the Study Group on Research Planning for Coal Utilization and Synthetic Fuel Production, Rev. Mod. Phys. 53 No 4, Oct. 1981 
(II-7) B. Cooper, ed.: "Scientific Problems of Coal Utilization", Proc. Conf. at Univ. of West Virginia, May 1977 CONF-770509

CHI'TER III

(SQ-1) Cornish, R.J.: "Flow in a Pipe of Rectangular Cross Section", Proc. Roy. Soc. A120 691 (1928)

(SQ-2) Lea,F.C. \& Tadros, A.G.:"Flow of Water through a Circular Tube with a Central Core and through Rectangular Tubes.", Phil.Mag. 11235 (1931)

(SQ-3) Kosicki, W., Chou, C.H. \& Tiu, C.: "Non-Newtonian Flow in Ducts of arbitrary cross-sectional shape", Chm. Eng.Sci. 21665 (1965)

(SQ-4) Wohl, M.H.:"Dynamics of Flow between Parallel Plates and in Noncircular Ducts", Chm. Eng. 183, May 1968

(SQ-5) Schecter, R.S.: "On the Strady Flow of a Non-Newtonian Fluid in Cylinder Ducts", A. I.Chm. Eng. Jor. I 445 (1961)

(SQ-6) Wheeler,J.A. \& Wissler,E.H.: "The Friction Factor-Reynolds' Number Relation for the Steady Flow of Pseudoplastic Fluids through Rectangular Ducts:, A. I.Chm. Eng. Jour. 11207 (1965)

(SQ-7a)Press, W.H., Flannery, B.P., Teukolski, S.A. \& Vetterling, W. T. : Numerical Recipes - the Art of Scientific Computing", Cambridge 1989

(SQ-7b)Southwell, R.V.: "Relaxation Methods in Theoretical Physics", McMillan, NY 1946

(SQ-8) Stanton, R.: "Numerical Methods for Science and Engineering", Prentire:Hall, Englewood Cliffs, NJ 1961

(SQ-9) Scarborough, J. B.: "Numerical Mathematical Analysis", Johns Hopkins, Baltimore 1958

(SQ-10)Forsythe, G. \& Wasow, W.R.: "Finite Difference Methods for Partial Differential Equations", Wiley, NY 1960

(F-1) Rouse, H. \& Ince, S.: "History of Hydraulics", Iowa Inst. of Hydr., Iowa City 1959

(F-2) Navier, L.M.H.: "Mémoire sur les Lois du Mouvement des Fluides", Mém. Acad.Roy.Sci. $\underline{6} 389$ (1823)

(F-3) Stokes, G.G.: "On the Theories of the Internal Friction of Fluids in Motion", Trans.Camb. Phil.Soc. 8 (1845)

$(F-4)$ Wilkinson, W. L.: "Non-Newtonian Fluids", Pergamon, NY 1960) 
(F-5) Dodge, D.W. \& Metzner, A.B.: "Turbulent Flow of Non-Newtonian Systems", A. I.Chm. Jour. 5189 (1959)

(F-6) Bogue, D.C.\& Metzner, A.B.: "Velocity Profiles in Turbulent Pipe Flow", I.\& E.C.Fund. 2143 (1963)

(F-7) Li, W.H. \& Lam, S-H.: "Principles of Fluid Merchanics", Addison-Wesley, NY, 1976

(F-8) Bird, R.B., Stewart, W.E. \& Lightfoot, E.N.: "Transport Phenomena", J. Wiley, NY 1960

(F-9) Li, W.H. \& Lam, S.H.: "Principles of Fluid Mechanics", Addison-Wesley, Reading, MA 1976

(F-10) Govier, G.W. \& Aziz, K.: "The Flow of Complex Mixtures in Pipes", Van Nostrand, NY (1972)

(F-11) Zandi, I. (ed): "Advances in Solid-Liquid Flow in Pipes and its Application", Pergamon, NY 1971

(F-12) Chereminisoff, N.P. \& Gupta, R. (eds): "Handbook of Fluids in Motion", Ann Arbor Science (1985)

(F-13a)Cheng,D.C.: "Viscosity-Concentration Equations and Flow Curves for Suspensions", Chem. \& Industry, May 1980

(F-13b)Metzner, A. B.: "Non-Newtonian Rheology", Adv. in Chem. Eng. I-77, Academic Press, NY (1956)

$(F-13 C)$ Thomas, D.G.: "Non-Newtonian Suspensions", Ind. \& Eng. Chem $\underline{55} 27$ (196.3)

\section{CHAPTER IV}

(G-1) Heitler, W.: "The Quantum Theory of Radiation", Cambridge 1936

(G-2) Jauch, J.M. \& Rohrlich, F.: "The Theory of Photons and Electrons", Springer, NY 1976

(G-3) Davisson, C.M., Ch II and App. I of Vol I, "Alpha, Beta and Gamma-Spectroscopy", K.Siegbahn ed.; North Holland, Amsterdam 1965

(G-4) Storm, E. \& Israel, H. I.: "Photon Cross sections from $1 \mathrm{KeV}$ to $100 \mathrm{MeV}$ for Elements $Z=1$ to $Z=100 ", L A-3753$ (1967)

(G-5) Fookes, R.A., Gravitis, V.L. \& Watt, J.S.: "Determination of Ash Content of Coal by Mass Absorption Coefficient Measurements at Two X-ray Energies", IAEA-SM-216/5, 167; Proc.Sympos. on Nucl. Tech. and Mineral Resources, Vienna 1977 
(G-6) Nagy, M. \& Varga, K.: "Determination of the Ash Content of Coal by Gamma Transmission", Proc. Sympos, on Rad. Instr. in Indust. \& Geophysics, IAEA, Warsaw 1969

(G-7) Pepelnik, R., Fanger, U., Michaelis, W. \& Bössow, E.: "Anwendung von Gamma-Strahlen beim Rohrleitungstransport", Rohre \& Rohrleitungsbau 17, $289(1978)$

(G-8) Kayray, INC, 516 W. Campus Dr., Arlington, IL 60606

(G-9) Ohmart Corp, 4241 Allendorf Dr., Cincinnati, OH 45209

(G-10) Texas Nuclear Inc, Box 9267, Austin, TX 78766

(G-11) Johnson, L.O., Wood, D.B. \& Lassahn, G.D.: "LOFT Advanced Densitometer for Nuclear Loss-Of-Coolant Experiments", ISA/ISBN 87664-443, 727(1979)

(E-1a) Moon, P. \& Spencer, D.E.: "Field Theory for Engineers", Van Nostrand, N.Y. 1961

(E-1b) Sackett, S. \& Healey, R.: "JASON - a Digital Computer Program for the Numerical Solution of the Linear Poisson Equation" UCRL-18721 (1969)

(E-2) Fenstermacher, M.E.: "A Study of Computer Techniques to Solve Poisson's Equation for mapping the Cross Section of a Multi-Electrode Capacitor with multiple Dielectric Regions", ANL 1978 (unpubl.)

(E-3) Shu, M.T. \& Weinberger, C.B.: "A Simple Capacitance Sensor for Void Fraction Measurement in Two-Phase Flow", Ind. Eng. Fundam. 2.1 175 (1982)

(E-4) Ekrann, S., Boe, A., Schmidt, F. Jacobsen, E., Time, R.W. \& Vat.ne, H., US Patent 4,751,842 (21 Jun 1988)

(E-5) Beck, M.S., US Patent 3,593,078 (23 Jul 1971)

(E-6) Beck, M.S., US Patent 3,636,763 (25 Jan 1972)

(E-7) Irons, G.A. \& Chang, J.S.: "Particle Fraction and Velocity Measurement in Gas-Powder Streams by Capacitance Transducers", Int.J. Multiph. Flow g 289 (1983)

(E-8) Prellwitz, S.B. \& Pysnik, J., US Patent 3,635,082 (18 Jan 1972)

(E-9) Gregory, G.A. \& Mattar, L.: "An In-Situ Fraction Sensor for Two-Phase Flows of Non-Electrolytes", J.Can.Pet.Tech. 1248 (1973)

(E-10) Albouelwafa, M.S.A. \& Kendal1, E.J.M.: "Analysis and Design of Helical Capacitance Sensors for Volume Fraction Determination", Rev.Sci. Instr. 50 872. (1979)

(E-11) Same Authors: "The use of Capacitance Sensors for Phase Percentage Determination in Multiphase Pipelines", IEEE. Trans. IM-29, 24(Mar 1980) 
(E-12) Merilo, M., Dechene, R.L. \& Cichowas, M.W.: "Void Fraction Measurement with a Rotating Field Conductance Gauge", J.of Heat Transf.g9 (2), 1977

(E-13) Thorn, R., US Patent 4,713,603 (15 Dec 1987)

(E-14) Porges, K.G.A., US Patent 4,899,101 (6 Feb 1990)

(E-15) Managan, W.W., Eichholz, J.J. \& Doering,R.W.: "Field Test of a Capacitive Transducer on the HYGAS Pilot Plant Coal Feedline", (I-2c) ff.

(E-16) Bobis, J.P., Sheen, S.K., Porges, K.G.A., Karplus, H.B.,Farahat, M.M. \& Brewer, W.E.: "Preliminary Report on Development of Non-Intrusive Flow Instrumentation at the ANL SLTF", ANL $\backslash F E-83-18$ (1983)

(E-17) Bobis, J.P., Porges,K.G.A., Brewer,W.E. \& Bernovich, L.T.: "Particle Velocity \& Solid Volume Fraction Measurements with a New Capacitive Flow Meter at the ANL S/GTF", ANL/FE-86-4 (1986)

(E-18) Mosotti, O.F.: "Effetti di Polarisazione nelle Materie sull'Influenza di un Campo Elettrico", Mem.Soc. Ital.Sci. (Modena) 14, 49 (1850)

(E-19) van Beek, L.K.H.:"Dielectric Behaviour in Heterogeneous Systems", Prog. Diel. I (Birks, J.B., ed.), 69; Heywood Books, London 1967

(E-20) Bottcher, C.J.F.: "Theory of Electric Polarization", 2d ed., Elsevier, Amsterdam 1972

(E-21) Maxwel1, J. C.: "Treatise on Electricity and Magnetism", 3d ed. Cambridge 1904

(E-22) Rayleigh, G. S.: "On the Influence of Obstacles Arranged in Rectangular Order upon the Properties of a Medium", Phil. Mag. 34, 481 (1892)

(E-23) Runge, I.: "Zur Elektrischen Leitfähigkeit metallischer Aggregate", Z. Tech.Phys. $\underline{6}, 61$ (1925)

(E-24) Meredith, R.W. \& Tobias, C.W.: "Resistance to Potential Flow through a Cubic Array of Spheres", J. Appl. Phys 31, 1270 (1960)

(E-25a)Molyneux, J.E.: "Effective Permittivity of a Polycrystalline Dielectric", J. Mathemat. Phys. 11, 1172 (1970)

(E-25b)Brown, W. F.: "Dielectric Constants of Non-Polar Fluids", J. Chem. Phys. $\underline{18}, 1193$ \& 1200 (1950); "Solid Mixture Permittivities",J.Chem. Phys. 23, $1514(1955)$

(E-26) Bruggeman, D.A.G.: "Berechnung verschiedener Physikalischen Konstanten von heterogenen Substanzen", Annal. Phys. 24, 636 (1935)

(E-27) Landauer, R.: "The Electrical Resistance of Binary Metallic Mixtures", J. Appl. Phys. 23, 779 (1952) 
(E-28) Looyenga, H.: "Dielectric Constants of Heerogeneous Mixtures", Physica 31, 401 (1965)

(E-29) Landau, L.D. \& Lifschitz, E.M.: "Eletrodynamics of Continuous Media", Pergamon, Oxford 1960

(E-30a)Harms, A.A. \& Forrest, C.F.: "Dynamic Effects in Radiation Diagnosis of Fluctuating Voids", Nucl. Scj. Eng. 46408 (1971);

(E-30b)Harms, A.A. \& Laratta, F.A.R.: "The Dynamic Bias in Radiation Interrogation of Two-Phase Flow", Int. J. Heat \& Mass Transf. 16 No 7 (1973)

(E-31) Porges, K.G. \& Doering, R.W.: "Mass Flowrate Measurement through Gamma Transmission-Correlation Processing", (I-2a) ff.

CHAPTER V

(D-1a) Heidrick, T.R. \& Satvold, J.R.: "Cross Section-Averaged Density and Mass Flux Measurements in Two-Phase Flow through Pipes", Measurements in Polyphase Flows, ASME $1978 \mathrm{p} 1$

(D-1b) Kondic, N.N. \& Hahn, O.J.: "Theory and Application of the Parallel and Diverging Radiation Beam Method in Two-Phase Systems", IVth Internat 1. Conf. on Heat Transfer, Paris 1970

(D-2) Korbel, K., Plechta, J. \& Przewlocki, K.: "Investigations of Kinematic Behaviour of Hydromixtures Flowing in Horizontal Head Pipelines", Jour. Hydrol. Sci. 4, 37 (1977)

(D-3a) Michalik, A, Praxmeier. T. \& Przewlocki, K.: "A Radiometric Method determining Spatial Distribution of Density in Closed media". Nukleonika 13, 107 (1968)

(D-3b) Michalik, A.: "Density Patterns of Inhomogeneous Mixtures in Industrial Pipe Lines Measured by Radiometric Scanning", La Houille Blanche 1, 53 (1973)

(D-4) Liptak, B (ed): "Instrument Engineer's Handbook", Chilton, NY (1969)

(D-5) Doebelin, E.O,: "Measurement Systems: Application and Design", Mc GrawHill, NY (1970)

(D-6) Davis, A.: "A Discussion of Some of the Methods of Physical Characterization Used for Coal", p 13, "Scientific Problems of Coal Utilization", B. R. Cooper, ed., DOE Sympos. Ser. $\underline{46}$ (1978)

(D-7) Porges. K. G.: "The Performance of a Light Pulser-operated Digital Stabilizer", Nucl. Inst \& Meth. 124, 235 (1975)

(D-8) Ruch, R.R., Gluskoter, H.J. \& Shimp, N.F.: "Occurrence and Distribution of Potentially Volatile Trace Elements in Coal", Illi. State Geol. Survey, Urbana, IL 1974 
(D-9) Abernethy, R.F., Peterson, M.J. \& Gibson, F.H.: "Major Ash Constituents in US Coals", USDI, Bureau of Mines, 1969

CHAPTER VI

(V-1) Fitzgerald,J.W. \& Doolittle, R.D.: "Feasibility Studies of Acoustic Flow Meters for Coal. Conversion Programs", Task II, Contract 31-109-3807, ANL/CT (1977)

$(V-2)$ ref. $(E-15)$, p. $63 \mathrm{ff}$.

(V-3) Faddick, R., Pouska, G., Connery, J., diNapoli, L. \& Punis, G.: "Ultrasonic Velocity Meter", Hydrotransport 6, paper B4 (1979)

(V-4a) Karplus, H.B., US Patent 4,413,531 (Nov 1983)

(V-4b) Karplus, H.\& Canfield, D.R.: "Development and Testing of a High-Temperature Acoustic Dopper Flow Meter", ANL/FE 81-64 (Sept 1981)

(V-4C) Karplus, H.B., Lee, S. \& Simpson, T.: "The ANL Doppler Flowmeter", ANL/ FE-85-14 (1985)

(V-5) Fitremann, J.M.: "Théorie des Vélocimètres et Débitmètres Électromagnétiques en Écoulement Biphasique", CR. Acad. Sci. 274 ser C, 440 (1974),

$(V-6 a)$ Theenhaus, R.: "Modern Developments and New Applications of Magnetic Flow Meters", Mod.Dev.Flow Meas., C.G.Clayton, ed., PPL Conf 10, 347 P.Peregrinus, London 1972

(V-6b) Shercliff, J.A.: "The Theory of Electromagnetic Flow Measurement", Cambridge 1962

(V-7a) Mills, R.C.\& Mannherz, E.D.: "A New Concept in Magnetic Flow Meter Design". Advances in Instrumentation 37 pt 3,1115 (1982)

(V-7b) Banta, F.D.: "Magnetic Flowmeter Development", ibid., 1183

(V-8) Plache, K.: "Coriolis/Gyroscopic Flow Meter", Mech. Eng. Mar. 1979, p.36

(V-9) Replogle, J.A., Myers, L.E. \& Brust, K.J.: "Flow Measurements with Fluorescent Tracers", J. Hydraul. Div., ASCE HY-5, 1 (1966)

(V-10) Allen, C.M. \& Taylor, E.A.: "The Salt-Velocity Method of Water Measurement", Trans. ASME $\underline{45}, 298(1928)$

(V-11) Hul1, D.E.:"The Total Count Technique: A New Principle in Flow Measurement", Int. J. Appl. Rad. Iso. ㄴ, 1(1958)

(V-12) Clayton, C.G., Spackman, R.\& Bell, A.M.: "The Accuracy and Precision of Flow Measurement by Radioactive Isotopes", Symp. Rad. Trac. in Industry \& Geophysics, IAEA, Prague 1966. 
(V-13) Clayton, C.G.: "Measurement of Flow of Liquids and Gases using Radioactive Isotopes", Iso. \& Rad. Tech. 1,93 (1966)

(V-14) Sauerwein, K., Hossner, R. \& Rotter, W.: "Radioaktive Isotope im Bergbau", Atomwirtschaft 1, 71 (1956)

(V-15) Fortier, A. \& Chen, C.P.: "Ecoulement Turbulent Stationnaire Biphasique Air-Solide dans un Tube Cylindrique à forte Concentration Massique", Jour. de Méc. 15, 155 (1976)

(V-16) Dunn, T.S. \& Gardner,R.P.: " The Development of Radiotracer Methods for Laminar Flow Measurements in Smal1 Channels: II. The Tracer-Sphere Method", Int.J.App.Rad. Iso 28, 355 (1977); "III. The Liquid Tracer Method", ibid. . 369

(V-17) Porges, K.G.A.: "Flow Characterization and Calibration of Slurries by Pulsed Neutron Activation", ANL/FE-84-16 (1984)

(V-18) Porges,K.G., Cox,S.A., Doering, R.W., Kampschoer,C.J. \& Herzenberg, C. : "Calibration of Flow and Feed Rate Meters In Situ by means of Pulsed Neutron Activation", ( I-2c) ft

(V-19) Porges, K.G.: "On-1 ine Mass Flow Measurement by Non-Intrusive Nuclear Techniques", Trans. ANS $\underline{45}, 762$ (1985)

(V-20) Porges, K.G., Cox, S.A., Herzenberg, C. \& Kampschoer, C.: "Flow Speed Measurement and Rheometry by Pulsed Neutron Activation", Jour. Fluids Eng. 111, 337 (1989)

(V-21) Taylor, G. I.: "The Dispersion of Matter in Turbulent Flow through a Pipe". Proc. Roy. Soc. Lond. A-223, 446 (1954)

$(V-22)$ Aris, R.: "On the Dispersion of a Solute in a Fluid Flowing through a Tube", Proc. Roy. Soc. Lond. A-237, 67 (1956); ibid. A-252, 538 (1959)

(V-23) Boswell, C.R. \& Pierce, T.L.: "Flow Rate Determinaton by Neutron Activation Analysis", Mod. Dev. Flow Meas., C.G. Clayton, ed., PPL Conf 10 , 264; P.Peregrinus, London 1972

(CC-1) Mitra, S.N.: "A Radio Method of Measuring Winds in the Ionosphere", Proc. IEEE 96, 441 (1946)

(CC-2) Randal1, R.L., Pekrul, P.J. \& Grayban, G.R.: "Development of Noise Analysis Techniques for Measuring Reactor Coolant Velocities", NAA - SR 11193 (1966)

(CC-3) Bogert,B.P., Healy,M.J.R. \& Tukey, J.W.: "The Quef rency Alanysis of Time Series", Proc.Symp. on Time Ser.Anal., M.Rosenblatt, ed, Wiley NY 1974

(CC-4) Cooley, J.W. \& Tukey, J.W.: "An Algorithm for the Machine Calculation of Complex Fourier Series", Math. Comp. 19, 297 (1965) 
(CC-5) Gold, B.\& Rader, C.M.: "Digital Processing of Signals", Mc Graw-Hill NY (1976)

(CC-6) Cooley, J.W., Lewis, P. \& Welch, P.: "The Fast Foruier Transform Algorithm and its Applications", IBM RC-1744 (1967)

(CC-7) Stockham, T.G.: "High-Speed Convolution and Correlation", Proc.AFIPS 28 , $229(1966)$

(CC-8) Nishihara. H. \& Konishi, H.: "A New Correlation Method for Transit-time Estimation", 2d. Spec. Meetg. on Reactor Noise, Gatlinburg, TN 1977

(CC-9) Pacilio, N.: "Reactor Noise Analysis in the Time Domain", USAEC TID24512 (1969)

(CC-10)Beck, M., Jordan, J.R. \& Kelly, R.G.: "Cross-Correlation Flow Measurement using a Microelectronic Hard-Wired Programmed Digital Computer", IEEE Conf. on the Use of Digital Computers in Measurement, IEEE Conf.P. 103, 104 (1978)

(CC-11)Soucek, B. : Microprocessors and Microcomputers",Wiley, NY 1976

(CC-12) TRW Inc, PO Box 2472, La Jolla, CA 92038

(CC-13)Bendat,J.M. \& Piersol,A.G.: "Measurement and Analysis of Random Data", John Wiley, NY 1960

(CC-14)Lange, F.: "Correlation Techniques", Iliffe, London 1967

(CC-15)Mesch, M. , Fritsche,R. \& Kipphan,H.:"Transit Time Correlation, a Survey of its Applications to Measuring Transport Phenomena", Trans. ASME, DeC 1974 , p. 414

(CC-16)Kipphan,H. \& Mesch,F.: "Flow Measurement of Fluids", H.H.Dijstelbergen, E.A.Spencer, eds., p $409 \mathrm{ff}$., North-Holland 1978

(CC-17)Le Guennec, B., Alquier, M., Santos-Cottin, H. \& Margrita,M.: "Analysis of a Horizontal Solid-Liquid Pipe Flow by a Cross-Correlation Method", Int. J. Multiph. Flow $\underline{4}, 511$ (1978)

(CC-18)Beck,M., Mendies, J. \& Gatland, H.B.: "Measurement and Control in Hydraulic Transport Systems using Cross Correlation Measurement and Fluidic Diverters", Hydrotransport $\underline{3}$, 3d Int. Conf. on Hydr. Trspt. of Solids in Pipes, 1974

(CC-19)Beck, M., Drane, J., Plaskowski, A. \& Wainwright, N.: "Particle Velocity and Mass Flow Measurement in Pneumatic Conveyors", Powder Tech. 2,269 (1968)

(CC-20)Beck,M. \& Ong, K.H.: "Slurry Flow Velocity, Concentration and Particle Size Measurement using Flow Noise and Correlation Techniques", Meas. \& Control 8453 (1975) 
(CC-21)Beck, M.: "Correlation in Instruments: Correlation Flowmeters", J.Phys. E. Sci. Instrum. 14,7 (1981)

(CC-22)Boonstoppel, F., Veltman, V. \& Kerngrouwen, F.: "The Measurement of Flow by Cross Correlation Techniques", Proc. Conf. on Ind. Tech. for Online Computers, London 1968, IEE Conf. Publ. $\underline{43}$ (June 1968)

(A-1) Petroff, M. D. \& Doggett, W. O.: "Application of a Statistical Correlation Function for Determining Daughter Half-lives in the Range $10^{-4}$ to $10^{-1}$ second", UCRL-3347 (1955), Rev. Sci. Instr. 27, 838 (1956)

(A-2) Doggett, W. O.: "A Time-Correlation Technique for Investigating Three Connected Nuclear Transformations", Inst. Int. de Statistique 32, paper 42, Tokyo (1966)

(A-3) Williams, A. \& Sara, R. I.: "Correlation Counting Applied to the Determination of Absolute Desintegration Rates", Nucl. Inst. \& Meth. 60, 189 (1967)

(A-4) Dumont, A. M. \& Camhy-Val, C.: "Data Analysis in Time-Correlation Mean Lifetime measurements", Nuc1. Inst. \& Meth. 106, $\underline{413}$ (1973)

(A-5) Seifritz, W. \& Stegemann, D.: "An On-Line Reactivity Meter Based on Reactor Noise Using Two-Detecor Cross-Correlation", Nucl. Appl. $\underline{6}, 209$ (1969)

$(A-6)$ Buhl, A.R. \& Ackermann, N.J.: "Precision of Shutdown Measurements Using the Two-Frequency Reactor Noise Technique in an LMFBR", IEEE. Trans. Nucl. Sci. NS-18\#1, 430 (1971)

(A-7) Williams, A., Lewis, V. E. \& Smith, D.: "Correlation Techniques in Radioactivity Measurement", Nucl. Inst. \& Meth. 112, 285 (1973)

(A-8) Tilli, K. J.: "Correlation in the Statistical Analysis of a Reverse Fourier Neutron Time-Of-Flight Experiment", Nuc. Inst. \& Meth. 192, 387 (1982)

(A-9) Fanger, U., Pepelnik, R. \& Michaelis, W.: "Determination of ConveyorFlow Parameters by Gamma-Ray Transmission Analysis", Proc. Sympos. on Nucl. Tech. and Mineral Resources, Vienna 1977 IAEA-SM-216-40, p.539

(A-10) Frisch, O.R., AECL-5 (1947)

(A-11) Cramer, H.: "Mathematical Methods of Statistics", Princeton 1949

(A-12) Veltman, B.P.: "Quantisierung, Abtastfrequenz und statistische Streuung bei Korrelationsmessungen", Regelungstechnik 14, 151 (1966)

(A-13a)Bazerghi, H. \& Serdula, K.J.: "Estimation and Reduction of Errors in Flow Measurements which use Cross-Correlation Techniques", Prog. Nucl. En. 1, 629 (1977) 
(A-13b)Bazerghi,H.: "Mesure de Débit d'un Liquide utilisant deux Sondes Ultrasoniques et la Téchnique de Correlation". Thèse, M.S.; Ec. Poly., Montréal, 1977

(A-14) Beck, M.S., Coulthard, J., Hewitt, P.J. \& Sykes, D.: "Flow Velocity and Mass Flow Measurement Using Natural Turbulence Signals", Mod. Dev. In Flow Meas., C.G. Clayton, ed.; PPL Conf Publ. 10, P. Peregrinus, London 1971; P 301, APPENDIX.

(A-15) Flemons, R. S.: "A New Non-Intrusive Flowmeter", Proc. Sympos. on Flow Meas, in Open Channels \& Conduits, L.K. Irwin, ed., NBS, Gaitherburg, MD (1977) 


\title{
APPENDIX A
}

\author{
INTRINSIC STATISTICAL ERROR IN CROSS-CORRELATION-BASED MEASUREMENT
}

\section{(1) INTRODUCTION}

Like other, more straightforward measurement schemes, Cross Correlationbased measurements involve both systematic and statistical errors; unlike other schemes, each one of a number of applications of that statistical scheme involves a method-specific, additional error that is treated in this Appendix. Systematic error is present in any kind of physical measurement, as is the other generic type, "statistical" error: characteristic of repeated measurements, this component error is subject to improvement through additional replications of a measurement; the effect of fluctuations in the measured variable on the calculated result can be traced through propagation.

In inferring the flow speed from a calculation of the Cross Correlation Function (CCF) with samples from an Upstream/Downstream sensor pair, two systematic errors, that of the instrument and that of the manner in which the instrument is read, have been repeatedly stressed in the preceding report: the "instrument" error being caused by sensing bias, "reading method" error resulting from data filtering and peak-reading. in this specific application of Cross-Correlation. When the method of measurement is based on statistical reasoning, a third generic type of error comes into play.

CCF (Cross Correlation Function) - based calculation and data acquisition has been applied in certain nuclear and reactor physics problems where alternative direct methods are severely impacted by statistical error: for example. as a substitute for delayed-coincidence counting in the measurement of millisecond nuclear decay (A-1)-(A-4); applications in reactor kin tics are likewise alternatives to hardware electronics schemes $(\mathbf{A}-5),(\mathbf{A}-6)$. The supposition that Cross-Correlation of fered better statistics than direct delayed-coincidence measurements for the same time span was not substantiated by further investigation $(\mathbf{A}-\mathbf{7})$, which indicated that both methods yielded an equivalent statistical quality of information (as indeed predicted by information theory(A-8)). Similar conclusions were reached with regard to the response of critical assemblies whenever alternative means were available.

Statistical quality of CCF processing may be worsened by the superposition of another "layer" of statistics on those of the CCF calculation per se, e.g., when Gamma transmission is used for purposes of CCF velocimetry. Other sensing methods that have benign intrinsic statistics thus offer better overall performance. These matters are reviewed below, in sufficient detail to obtain quantitative estimates of the Fractional statistical Error (FSE). The approach adopted here is based directly on statistics and probability theory, more specirically on refs $(\boldsymbol{A}-\mathbf{1 0})-(\mathbf{A}-\mathbf{1 2})$; combinatorial statistics are treated in refs. $(\mathbf{A}-\mathbf{1})$ and $(\mathbf{A}-\mathbf{A})$.

The existing literature on CCF velocity measurement, consisting largely of "feasibility studies", does not offer much help in statistical evaluation: error is usually negiected altogether while those few reports that ment ion the topic at all offer the reader a variety of quite different formulae, based on arguments that are not developed explicitly. More thorough and consistent er- 
ror treatments, developed for the other cross-correlation schemes mentioned above, cannot be directly applied to velocity measurement.

\section{(II) SIMPLIFIED MODEL AND COMBINATORIAL STATISTICS}

To avoid complexity in a purely statistical estimate, it is expedient to adopt a simplified model in which detector length $\Delta z$ is small, there are no clusters and the velocity distribution is narrow, such that both the ACF and the CCF are limited to single sample-width. For this model, upstream (subscript $u$ ) and downstream (subscript d) Solid Volume Fraction (SVF) samples $\phi$, of sample number $i, i+k$ respectively are related as follows:

$$
\phi_{d, 1+k}=K \phi_{u, 1}+(1-K) \phi_{1}^{*} \quad(A-1)
$$

where $\mathrm{K}$ is the correlation coefficient or fading constant, of the foregoing; the starred SVF in the second term represents the uncorrelated component of the ith sample. Averaging (denoted by $\langle\phi\rangle$ ) over a large number $N$ of consecutive samples (or, over a time $T=N \tau, \tau=$ sampling interval),

$$
\phi_{u}, \phi_{d}, \phi^{*},=\langle\phi\rangle .
$$

In practice, arithmetic manipulations are carried out with signal rather than SVF samples; the signal samples being certain functions of the SVF that vary with the sensing scheme. For the present, we wish to discuss the moments and mixed moments of SVF samples, for example, the CCF or mixed second moment: making use of Eq $(A-1)$, this can be written

$$
\begin{gathered}
\mathrm{CCF}=\frac{1}{\mathrm{~N}} \sum_{1}\left(\phi_{u, 1}-\langle\phi)\left(K\left[\phi_{u, 1}-\langle\phi\rangle\right)+(1-K)\left(\phi_{1}^{*}-\langle\phi\rangle\right)\right.\right. \\
=K \operatorname{var}(\phi),
\end{gathered}
$$

neglecting terms $<1 / \mathrm{N}$. As further discussed below, the same statistical considerations apply to the signal samples U,D.

To evaluate higher moments, we must consider how these moments are related to their corresponding sample products:

Amongst all $\mathrm{N}^{\mathrm{q}}$ products of $\mathrm{q} \mathrm{N}$-sample sums,

$\mathrm{N}$

$N(N-1)$

$N(N-1)(N-2)$

feature correlated subscripts.

feature correlated pairs, mutually uncorrelated,

feature correlated triplets, mutually uncorrelated, etc.

$N(N-1) \ldots(N-q+1)$, form a $q-d i m e n s i o n a l$ mat $r i v$.

For $q=2$, there are evidently $\mathrm{N}$ diagonal and $\mathrm{N}(\mathrm{N}-1)$ of $f-d i a g o n a l$ terms. As the various terms are computed, some turn out to be proportional to $1 / \mathrm{N}$, some to 
higher powers of $1 / \mathrm{N}$; since $\mathrm{N}$ is always a large number, only terms in $1 / \mathrm{N}$ need be retained.

These considerations may now be directly applied to obtain a quantitative estimate of the effect of sample combinatorics on the statistical quality of the CCF. Writing the variance of the CCF as the difference between the average of squared sums and the averaged CCF,

$$
\operatorname{var}\{\mathrm{CCF}\}=\left[\left\langle\left(\frac{1}{N} \sum U D\right)^{2}\right\rangle-\left\langle\frac{1}{N} \sum U D\right\rangle^{2}\right], \quad(A-5)
$$

we apply combinatorial grouping, finding, for example, that the mixed product of sums,

$$
\left[\frac{1}{\mathrm{~N}} \sum U \mathrm{D}\right]\left[\frac{1}{\mathrm{~N}} \sum \mathrm{U}\right]\left[\frac{1}{\mathrm{~N}} \sum \mathrm{D}\right]
$$

becomes, upon evaluation, a set of three terms of which:

the first term, $\frac{1}{N^{2}}\left\langle U^{2} D^{2}\right\rangle$, combines all elements in a single group,

the second term, $\left.\frac{N-1}{N^{2}} \mid\langle U D\rangle^{2}+\langle D\rangle\left\langle U^{2} D\right\rangle+\langle U\rangle\left\langle U D^{2}\right\rangle\right]$, includes all double groups,

the third term, $\frac{(N-1)(N-2)}{N^{2}}\langle U D\rangle\langle U\rangle\langle D\rangle$, features three groups.

Keeping only terms of order $\mathrm{l} / \mathrm{N}$ or larger, and similarly evaluating all other terms of Eq $(A-5)$, one thus obtains its explicit version,

$$
\begin{aligned}
\operatorname{var}\{\mathrm{CCF}\} & =(1 / N)[(\mathrm{I})+(\text { II })-(\text { III })+(\text { IV })] ; \\
\text { (I) } & =\left\langle U^{2} D^{2}\right\rangle-\langle U D\rangle^{2} \\
\text { (II }) & =6\langle U\rangle\langle D\rangle(\langle U D\rangle-\langle U\rangle\langle D\rangle) \\
(\text { II }) & =2\langle U\rangle\left[\left\langle U D^{2}\right\rangle-\langle U\rangle\langle D\rangle^{2}\right]+2\langle D\rangle\left[\left\langle U^{2} D\right\rangle-\langle U\rangle^{2}\langle D\rangle\right] \\
\text { (IV) } & =\left[\left\langle U^{2}\right\rangle-\langle U\rangle^{2}\right]\langle D\rangle^{2}+\left[\left\langle D^{2}\right\rangle-\langle D\rangle^{2}\right]\langle U\rangle^{2}
\end{aligned}
$$

as can be verified, with a certain amount of arithmetic. Eq. (A-6) is of fundamental significance in that it furnishes the basis for the statistical evaluation of all cross-correlation schemes, details varying with the specific sensor model, as will now be considered.

\section{I MOMENTS FVALUATION FOR CAPACITIVE SENSING}

Evaluation of the terms I-IV for capacitive sensing requires the relation between signal and SVF, discussed in Chapter III, 


$$
S_{1}=\frac{C_{m 1}-C_{E}}{C_{E}}=3 g \phi_{1}(1+g\langle\phi\rangle)^{2}+3 g^{2}(1+g\langle\phi\rangle)\left(\phi_{1}-\langle\phi\rangle\right)^{2}+g^{3}\left(\phi_{1}-\langle\phi\rangle\right)^{3}(A-7)
$$

where $S$ stands for $U$ or $D$. For purposes of variance estimation, we shall again avoid complexity and drop the second and third terms, thus obtaining a quasilinear relation between signal and SVF. Let $3 g(1+g\langle\phi\rangle)^{2}=a$; after considerable arithmetic (again omitted here), one finds, in terms of SVF moments

$$
\begin{aligned}
& m_{1}=\left\langle(\phi-\langle\phi\rangle)^{3}\right\rangle,(A-8) \\
& I= a^{4}\left\{\left[K^{2} m_{4}+\left(1-2 K^{2}\right) m_{2}^{2}\right]+2 K(K+1) m_{3}\langle\phi\rangle+2(1+K) m_{2}\left\langle\phi^{2}\right\rangle\right\} ; \\
& I I=6 a^{4} K m_{2}\langle\phi\rangle^{2}, \\
& I I I=-2 a^{4}\left\{K(1+K) m_{3}\langle\phi\rangle+2(1+2 K) m_{2}\langle\phi\rangle^{2}\right\}, \\
&: V=2 a^{4} m_{2}\langle\phi\rangle^{2},
\end{aligned}
$$

hence, dividing by $\mathrm{CCF}^{2}$ and taking the square root, one obtains the Fractional Statistical Error FSE $=\varepsilon$,

$$
\left.\varepsilon=\left[m_{1} / m_{2}^{2}+\left(1-2 K^{2}\right) / K^{2}\right] / N\right]^{1 / 2}
$$

It. now remains to interpret the SVF moments $m$, in terms of a model:

Let $V=$ detector slice volume $=\pi D^{2} \Delta z / 4, v=$ particle volume $=d^{3} \pi / t, v=$ number of particles sampled, $q=v / v=2 d^{3} / 3 D^{2} \Delta z$; then

$$
\begin{gathered}
\phi=n v=N q, \\
\mathrm{~m}_{j}(\phi)=\mathrm{q}^{3} \mathrm{M}_{j}(N) .
\end{gathered}
$$

Supposing that $N$ is Poisson-distributed, then

hence, the FSE comes to

$$
M_{2}=\langle N\rangle, M_{4}=3\langle N\rangle^{2}+\langle N\rangle
$$

$$
\varepsilon=(1 / \sqrt{ } N)\left[1+q /\langle\phi\rangle+1 / K^{2}\right]^{1 / 2} . \quad(A-10)
$$

Assuming the set of values

$$
\begin{gathered}
d=0.2 \mathrm{~cm}, D=5 \mathrm{~cm}, \Delta z=1 \mathrm{~cm}, \mathrm{q}=2 \times 10^{-4},\langle\phi\rangle=0.2, \mathrm{~N} \sim 10^{3}, \\
q /\langle\phi\rangle=10^{-3} .
\end{gathered}
$$

For a fading constant $K$ near unity, the FSE for this representative set of parameters is very small. Even when the several simplifying assumptions on which this derivation was based (a unique velocity and linear relation between 
signal and SVF) are allowed for, and further noting that the final error of the velocity determination still involves the error in the averaging process over the CCF, Eq. (A-10) strongly supports capacitive sensing from the statistical point of view. The overall error includes systematic errors that may have resulted from conventional, biased detection geometries but can be effectively eliminated by means discussed in this report.

\section{MOMENTS EVALUATION FOR GAMMA TRANSMISSION SENSING}

For Gamma transmission sensing in conjunction with CCF (Cross Correlation Function) velocity measurement, evaluation of the terms of $\mathrm{Eq}(\mathrm{A}-8)$ involves the Poisson distributions of both gamma sources, superposed on solids fluctuations.

Proof-of-principle CCF-velocity measurements based on gamma transmission sensing have been reported(A-9), however, without providing a discussion or treatment of the statistical error. In sketching out such a treatment. Eq (A$1)$ is used to model the SVF fluctuations; in the following, subscripts are omitted to simplify expressions.

Let $\mathbb{N}_{u}, \mathbb{N}_{d}=$ number of source photons that would impinge on the channel detector during the sampling interval $\tau$ in the absence of absorbers (duct and medium). Assume that mean source strengths have been carefully equalized:

$$
\left\langle\mathbb{N}_{u}\right\rangle=\left\langle\mathbb{N}_{d}\right\rangle=\langle\mathbb{N}\rangle .
$$

As discussed in Chapter IV, count moments $C$, are calculated by averaging,

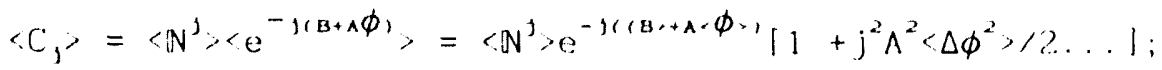

likewise, mixed moments,

$$
\begin{aligned}
& \left\langle C_{u} C_{d}\right\rangle=\left\langle\mathbb{N}_{u}\right\rangle\left\langle\mathbb{N}_{d}\right\rangle e^{\left.-2(\cdot B\rangle+A<\phi_{\nu}\right)}\left[1+A^{2}\left\langle\left(\Delta \phi_{u}+\Delta \phi_{d}\right)^{2}\right\rangle / 2 \ldots\right] \\
& =\mathbb{C}^{2}\left\{1+A^{2}\left[\left\langle\Delta \phi^{2}\right\rangle+\left\langle\Delta \phi_{u} \Delta \phi_{d}\right\rangle\right] \ldots\right\} \\
& \left.=\mathbb{C}^{2}\left\{1+A^{2}(1+K)<\Delta \phi^{2}\right\rangle\right\} \text {. }
\end{aligned}
$$

The CCF thus can be expressed in terms of the correlation coefficient $K$ and the SVF/photon transmission scaling constant A,

$$
\mathrm{CCF}=\mathbb{C}^{2} \mathrm{KA}^{2} \mathrm{~m}_{2},
$$

$\mathbb{C}$ being the photon \# counted after transmission through a quiescent medium of same composition,

$$
\mathbb{C} \times\langle\mathbb{N}\rangle e^{-(B+A+\phi,)} \text {. }
$$

The terms in Eq $(\mathrm{A}-8)$ can be expressed as averages over transmission factors 


$$
\begin{aligned}
& P_{x}=\exp -\left(A \Delta \phi_{x}\right), \quad x=u, d \\
& (1)=\mathbb{C}^{2}\left[\mathbb{C}^{2}\left[\left\langle\mathrm{P}_{u}^{2} \mathrm{P}_{d}^{2}\right\rangle-\left\langle\mathrm{P}_{u} \mathrm{P}_{d}\right\rangle^{2}\right]+\mathbb{C}\left[\left\langle\mathrm{P}_{u}^{2} \mathrm{P}_{d}\right\rangle+\left\langle\mathrm{P}_{u} \mathrm{P}_{d}^{2}\right\rangle\right]+\left\langle\mathrm{P}_{u} P_{d}\right\rangle\right], \\
& \text { ( I I })=6 \mathbb{C}^{4}<\mathrm{P}_{\mathrm{u}} \mathrm{P}_{\mathrm{d}}>\left\langle\mathrm{P}_{\mathrm{u}}>\left\langle\mathrm{P}_{\mathrm{d}}>\right.\text {, }\right.
\end{aligned}
$$

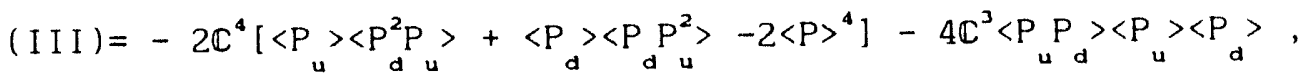

$$
\begin{aligned}
& \text { (IV) }=\mathbb{C}^{4}\left[\left\langle\mathrm{P}_{u}^{2}\right\rangle\left\langle\mathrm{P}_{\mathrm{d}}\right\rangle^{2}+\left\langle\mathrm{P}_{\mathrm{d}}^{2}\right\rangle\left\langle\mathrm{P}_{u}\right\rangle^{2}-2\left\langle\mathrm{P}_{\mathrm{u}}\right\rangle^{2}\left\langle\mathrm{P}_{\mathrm{d}}\right\rangle^{2}\right]+\mathbb{C}^{3}\left[\left\langle\mathrm{P}_{\mathrm{u}}\right\rangle^{2}\left\langle\mathrm{P}_{\mathrm{d}}\right\rangle+\left\langle\mathrm{P}_{\mathrm{d}}\right\rangle\left\langle\mathrm{P}_{\mathrm{u}}\right\rangle^{2}\right] .
\end{aligned}
$$

Explicitly, Term (I), for example, comes to

$$
(I)=\sum_{u} W\left(\mathbb{C}_{u}\right) \sum_{d} W\left(\mathbb{C}_{d}\right) G\left(\phi_{u}, \phi_{d}\right) d \phi_{u} d \phi_{d} F\left(\mathbb{C}_{u} \mathbb{C}_{d} \phi_{u} \phi_{d}\right) ;
$$

$W$ are Poisson distributions and $F$ involves dual Bernoulli distributions B:

$$
\begin{aligned}
F=\sum_{k=1} \mathbb{C}_{u} ! B\left(\mathbb{C}_{u}, \phi_{u}\right)[k+k(k-1)] \sum_{j=1} \mathbb{C}_{d} ! B\left(\mathbb{C}_{d}, \phi_{d}\right)[j+j(j-1)] \\
=\left[\mathbb{C}_{u}\left(\mathbb{C}_{u}-1\right) P_{u}^{2}+\mathbb{C}_{u} P_{u}\right]\left[\mathbb{C}_{d}\left(\mathbb{C}_{d}-1\right) P_{d}^{2}+\mathbb{C}_{d} P_{d}\right],
\end{aligned}
$$

upon averaging over the individual Poisson and joint SVF distributions. Collecting terms,

$$
\operatorname{var}\{\mathrm{CCF}\}=2 \mathbb{C}^{3} \mathrm{~A}^{2} \mathrm{~m}_{2}+\mathbb{C}^{2}\left[1+(1+K) \mathrm{A}^{2} \mathrm{~m}_{2}\right]
$$

hence,

$$
E=\left(1 / \sqrt{ } N_{C K A} m_{2}\right)\left[1+(2 \mathbb{C}+1+K) A^{2} m_{2}\right]^{1 / 2}
$$

To estimate the FSE, we again choose representative parameter values:

$\Delta z=0.2 \mathrm{~cm}$, hence, $\mathrm{q}=10^{-3} ;\langle\phi\rangle=0.4 ; \mathrm{A} \cong 0.15$ (for a coal-water mixture, cf. CH III); $K \sim 1$; suppose that pure Poisson statistics distribute $2 \mathrm{~mm}$ particles, then

$$
A^{2} m_{2}=A^{2} q\langle\phi\rangle=2.55 \times 10^{-5}\langle\phi\rangle \approx 10^{-5}
$$

At a $2 \times 10^{5} / \mathrm{s}$ count rate and $1 \mathrm{~ms}$ sampling interval, $\mathbb{C} \cong 200 ; 1 / \mathbb{C A}^{2} \mathrm{~m}_{2} \approx 500$.

Calculated on the basis of $N=4 \times 10^{6}$ samples (which require $4 \times 10^{3} \mathrm{~s}$ to collect), the statistical error (FSE) still comes to as much as $25 \%$. The example clearly demonstrates that Gamma transmission CCF sensing is statistically counterindicated, unless:

*Solids are coarser than $2 \mathrm{~mm}$, thus increasing $\mathrm{q} \sim \mathrm{d}^{3}$, 
* The particle distribution deviates significantly from the Poisson law, towards a Polya distribution (clusters etc.),

* The gamma transmission contrast $\Delta \rho$, only 0.4 for coal-water, is significantly stronger (as e.g., for a metallic ore-water mixture), thus increasing the scaling constant $A$,

* The sample $\mathbb{C}$ is significantly increased by abandoning photon counting (and thereby discrimination against secondary radiation, cf. Chapter III). The factor $q$ can also be increased by narrow-beam sensing, again adversely affecting accuracy.

Ref (A-10), it may be noted, applies gamma transmission sensing to a duct carrying coarse sea-bottom material (such as manganese nodules), featuring some particles of several $\mathrm{cm}$ diameter. Other reported uses of gamma transmission typically use current rather than count acquisition and narrow-beam sensing (both measures, while ameliorating the FSE, also increase the systematic error and are thus not necessarily beneficial).

As mentioned in the foregoing, overland coal-slurry pipelines may feature a fairly coarse consist; in-plant conveyors (especially reactor feedlines) are more likely to run a fine consist. For these conveyors, gamma transmissionbased CCF velocimetry is evidently counterindicated, even though gamma transmission densitometry is the most accurate and reliable amongst available on-line density/composition measurement schemes.

Amongst others, Vetter $(\mathbf{A}-12)$, Bazerghi(A-13) and $\operatorname{Beck}(\mathbf{A}-14)$ have given formulae for the evaluation of Statistical error in CCF Flow measurement. Of these, Bazerghi's treatment is the most explicit and provides comparisons with measurements using a CCF scheme designed by Flemons $(A-15)$. However, none of these treatments is based on modeling of the process, hence, none seerns to yield useful equations that allow numerical estimates. 


\section{APPENDIX B: SELECTED FORTRAN PROGRAMS}

\section{(1) LAMINAR FLOW VELOCITY CONTOURS IN A SQUARE DUCT}

(a) \& (b) Velocity maps, Newtonian and Pseudoplastic

The first set of programs reproduced in this appendix solves the NavierStokes equation, as discussed in the foregoing text. The first program produces the velocity map for Newtonian Flow; that map is used as the initial approximation for the Pseudoplastic flow, index $m=0.9$ map - which in turn is used for $m=0.8$, etc. Maps are averaged and compared to the theoretical average velocity, related to the Centerflow velocity as discussed in Chapter [1. An adjustable multiplicative constant is varied and the calculation repeated until averaged and formula velocities agree. Details are more fully discussed in Chapter II. The Flow behaviour index $m$ together with the parametric viscosity $K$ allow a two-parameter approximation law for Pseudoplastic flow,

$$
\tau=K(d V / d n)^{m}
$$

that relates scalar stress $\tau$ to the normal velocity gradient. Though lacking a thorough theoretical foundation in terms of microscopic phenomena, this law has found wide acceptance and is thus used throughout this report.

(a) Laminar-Newtonian Velocity Map. Square Duct

C SQDCT1.FOR VELOCITY MAP, LAMINAR-NEWTONIAN FLOW IN A SQUARE DUCT

\$NODEBUG

\$NOFLOATCALLS

DOUBLE PRECISION GFAC $(60), \operatorname{VAV}(00), \operatorname{VBER}(60), \mathrm{VBAR}, \mathrm{VER}$

REAL*8 $V(65,65), U(36,36), X(36), Y(36)$

CHARACTER* 16 TRYNAME, DCTNAME, NUMNAME

DATA N, NN, NA, DEL, GF, VCT/33, 65, 1, 0.015625, 1. 0, 2. 10/

$C \operatorname{SET} V(I, J)=0, I=1, J=1 \ldots N N ; I=N N, J=1 \ldots N N$

DO $1 \mathrm{I}=1, \mathrm{NN}$

$V(I, 1)=0$

$V(I, N N)=0$

1 CONTINUE

DO $2 \mathrm{~J}=1, \mathrm{NN}$

$V(1, J)=0$

$\mathrm{V}(\mathrm{NN}, \mathrm{J})=0$

2 CONTINUE

C ( 1 ) CALCULATE INITIAL APPROX. V $(\mathrm{I}, \mathrm{J})$ FORMULA VALUES, IST QUADRANT

$\mathrm{VBAR}=\mathrm{VCT} / 2.25$

VER $=V B A R-1$.

$\mathrm{Q}=3 .{ }^{*} \mathrm{VCT} * \mathrm{DEL} * 2$

DO $4 \quad I=1, N$

$X(I)=$ DEL*FLOAT $(I-1)$

DO $3 \mathrm{~J}=1, \mathrm{~N}$

$Y(J)=$ DEL*FLOAT $(J-1)$

$V(I, J)=16 .{ }^{*} V C T * X(I) *(1 .-X(I)) * Y(J) *(1 .-Y(J))$ 
3 CONTINUE

4 CONTINUE

C (2) OPEN TRY FILE; HEADER, COPY 2 AD LINES TOP/RIGHT FOR ITERATION WRITE $(*, 60)$

60 FORMAT( $/ 8 X$, , Specify f i lename TRSNN. INF' ' $)$

READ (*, 99) TRYNAME.

$\operatorname{OPEN}(10$, FILE$=$ TRYNAME, STATUS=' NEW' $)$

WRITE $(*, 61)$ TRYNAME

WRITE $(10,61)$ TRYNAME

61 FORMAT(/18X,' SQ1H.FOR ITERATION FILE ',A12,/)

C RETURN TO 5 FOR NEXT PASS

5 WRITE $(*, 62) N A-1, V C T, V E R, G F, Q$

WRITE $(10,62)$ NA-1, VCT, VER, GF, Q

62 FORMAT ( $/ 20 \mathrm{X}$, ' PASS \#', I2,' PARAMETERS :', $/ 3 \mathrm{X},{ }^{\prime} \mathrm{VCT}={ }^{\prime}, \mathrm{F} 9.7,2 \mathrm{X}$,

$X^{\prime}$ VER $={ }^{\prime}, E 12.6,2 X,{ }^{\prime} \mathrm{GF}={ }^{\prime}, F 9.7,2 X,{ }^{\prime} \mathrm{Q}={ }^{\prime}, \mathrm{E} 12.7 / 1$

C COPY 1ST QUADRANT ONTO OTHER QUADRANTS: DEFINE. $V(I, J)$ FOR FULL DUCT

DO $7 \quad I=1, N-1$

DO $6 \mathrm{~J}=1, \mathrm{~N}$

$\mathrm{V}(\mathrm{N}+\mathrm{I}, \mathrm{J})=\mathrm{V}(\mathrm{N}-\mathrm{I}, \mathrm{J})$

6 CONTINUE

7 CONTINUE

DO $9 \mathrm{~J}=1, \mathrm{~N}-1$

DO $8 \quad I=1,2 * N-1$

$V(I, N+J)=V(I, N-J)$

8 CONTINUE

9 CONTINUE

IF (NA.GT. 1 ) GOTO 13

C (3) 60 BOUSTROPHEDON PASSES; UP/DN, RT/LEFT; SYMMETRIZE QUAD IDIAG

C AVG); DISPL-RECORD 8 CTLINE $V(I, N)$ EVERY 3D PASS.

WRITE $(*, 63)$

WRITE $(10,63)$

63 FORMAT $\left(3 X_{,}^{\prime}, V(5,33)^{\prime}, 1 X,{ }^{\prime} V(9,33)^{\prime}, 1 X,{ }^{\prime} V(13,33)^{\prime}, 1 X, ' V(17,33)^{\prime}\right.$

$\left.X 1 X,{ }^{\prime} V(21,33)^{\prime}, 1 X,{ }^{\prime} V(25,33)^{\prime}, 1 X,{ }^{\prime} V(29,33)^{\prime}, 1 X,{ }^{\prime} V(33,33)^{\prime} / 1\right)$

13 DO $22 \mathrm{KA}=1,60$

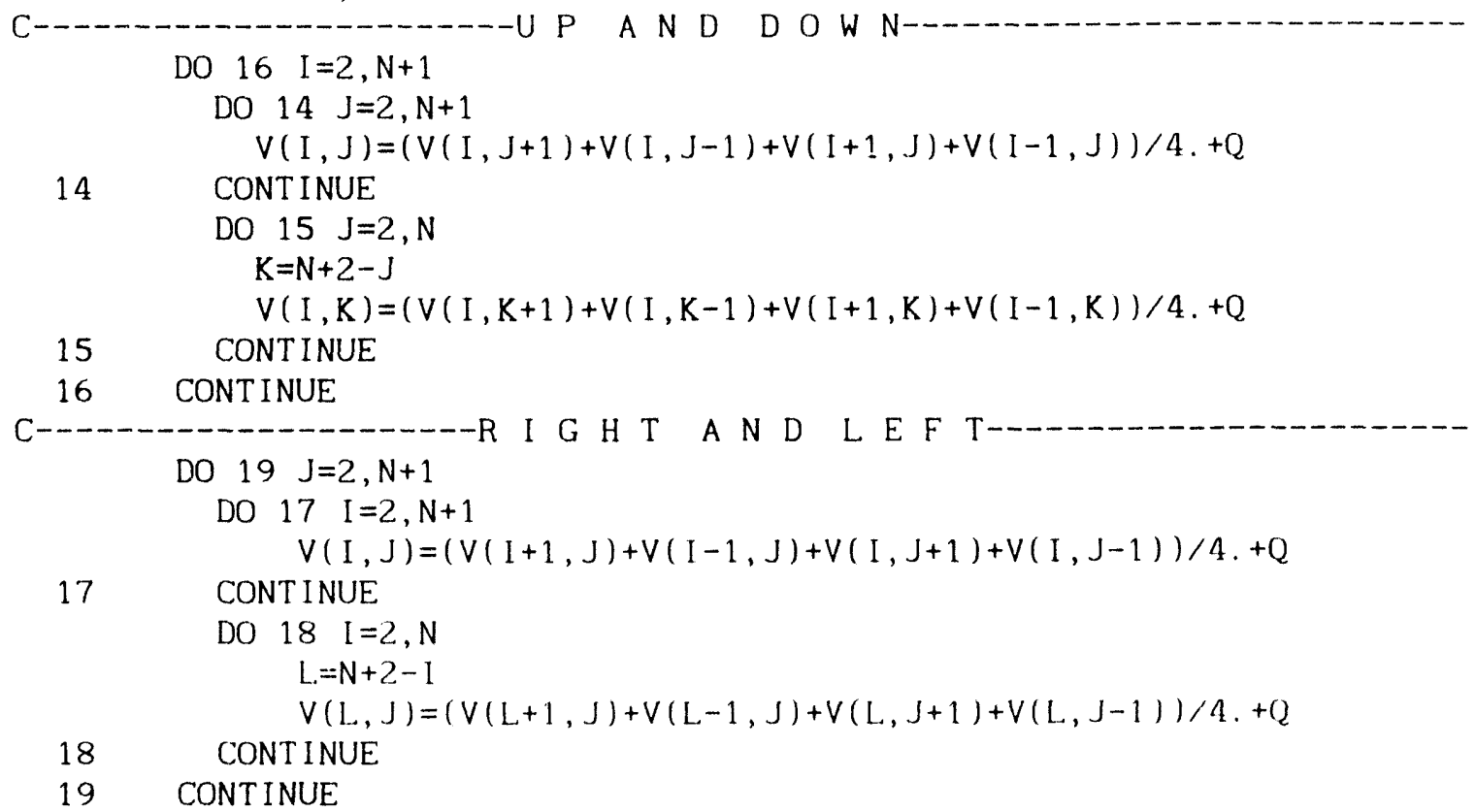




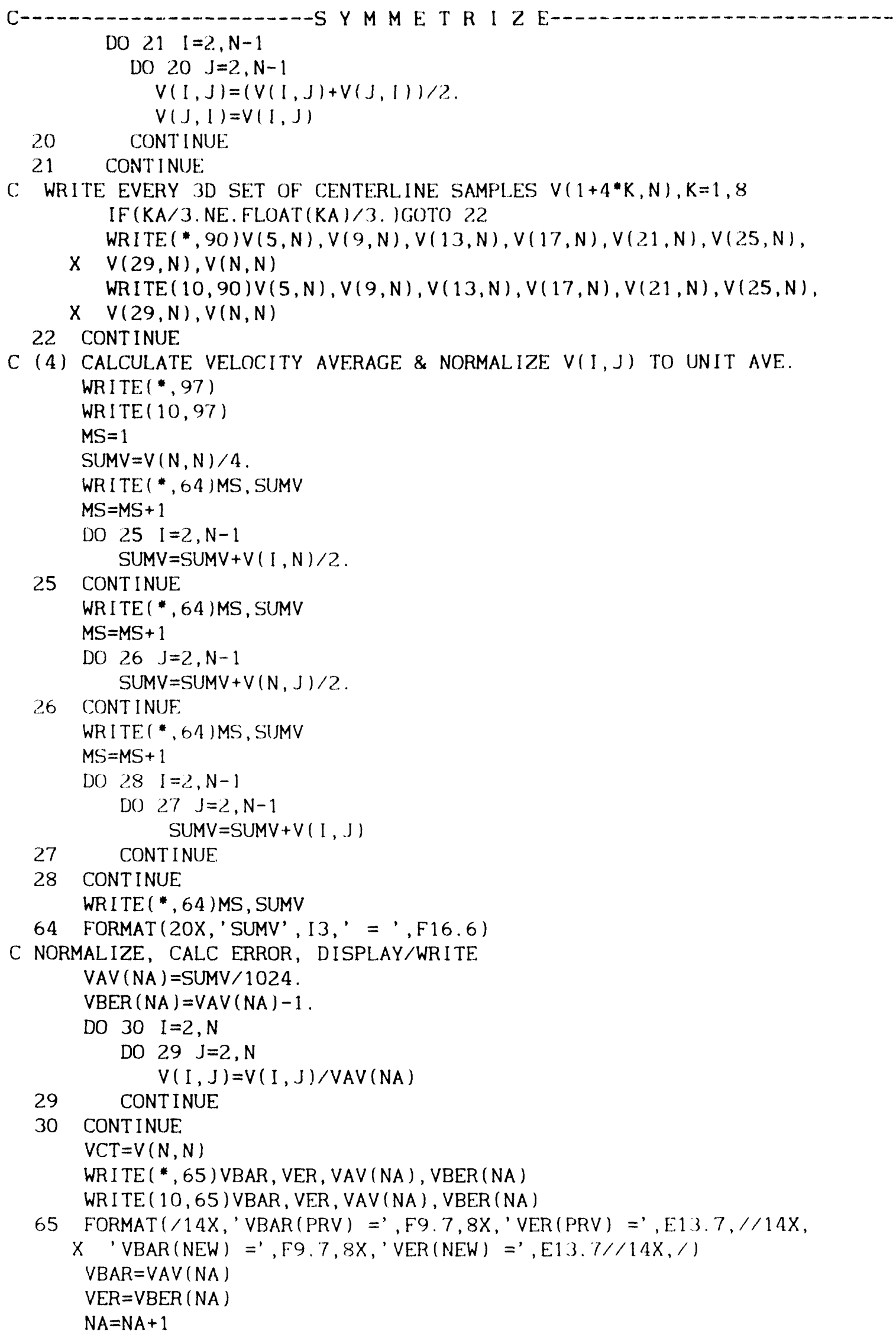


C (5) GF ADJUST OPTION; RECALCULATE Q; QUIT/RECYCLE OPTION WRITE (", 66)GF

66 FORMAT (8X,'G-FACTOR WAS: ',F9.7.2X,'; NFW G-FACTOR(F9.7): '\) READ(*,75)GFAC(NA)

75 FORMAT(F9.7) $Q=\operatorname{GFAC}(N A) *(9 . * \mathrm{~V}(33,2)-4.50 * V(33,3)+\mathrm{V}(33,4)) / 264$.

WRITE $(*, 67)$

67 FORMAT (/8X,' QUIT ? (0) REPEAT ? (1) '

READ(* " INX

IF (NX.EQ. O) GOTO 34

$\mathrm{GF}=\mathrm{GFAC}(\mathrm{NA})$

IF (NA.EQ. 60)GOTO 70

GOTO 5

70 WRITE $(*, 71)$

71 FORMAT( $/ 12 X$, 'TOO MANY ITERATIONS; QUIT(1)? WRITE anyway $(0)$ ? ' $)$ READ(*, ")NM

IF (NM. EQ. 1 )GOTO 100

C (6) CLOSE TRY FILE; REVERSE ABSC ISSA, WRITE INFO FILE \& NUM FILE

34 NMAX $=$ NA

WRITE $(10,62)$ NMAX, VCT, VER, GF, Q

WRITE( *, 62) NMAX, VCT, VER, GF, Q

$\operatorname{CLOSE}(10)$

WRITE $(*, 97)$

PAUSE'

$(5)^{\prime}$

C REVERSE ABSCISSA FOR NEXT STEP

DO $36 \quad I=1, N$

DO $35 \mathrm{~J}=2, \mathrm{~N}$

35 CONTINUE

36 CONTINUE

(: OPEN DCTS. INF FILE, WRITE (A) ITERATION SEQUENCE, (B) FINAL UI I,J) WRITE $(*, 68)$

68 FORMAT( $/ 12 X$,'Spec if y f i lename DCTSNN. INF ( $S=$ Index) , $)$

$\operatorname{READ}(*, 99)$ DCTNAME

OPEN ( 10, FILE=DCTNAME, STATUS=' NEW' $)$

WRITE $(10,98)$ DCTNAME

WRITE $(*, 98)$ DCTNAME

WR I TE $(10,62)$ NMAX, VCT, VER, GF , Q

WRITE $(10,69)$

WRITE $(*, 69)$

DO $37 \mathrm{NA}=1, \mathrm{NMAX}$

WRITE $(10,91) N A, \operatorname{VAV}(N A), \operatorname{VBER}(N A), \operatorname{GFAC}(N A)$

WRITE(, 91$)$ NA, $\operatorname{VAV}(N A), \operatorname{VBER}(N A), \operatorname{GFAC}(N A)$

37 CONT INUE

69 FORMAT ( $/ 7 \mathrm{X}$, ' $^{\mathrm{N}}$ ' , 6X, ' $\mathrm{V}($ AVE ) ' , 8X, ' ERROR \%', 8X, 'G-FACTOR' /)

$\operatorname{WRITE}(10,97)$

WRITE $(*, 97)$

$\operatorname{WRITE}(10,81)$

WRITE $(*, 81)$

DO $51 I=1, N$

$\operatorname{WRITE}(10,90) \quad(U(I, J), J=2,9)$

WRITE $(*, 90) \quad(U(I, J), J=2,9)$

51 CONTINUE

$\operatorname{WRITE}(10,82)$ 


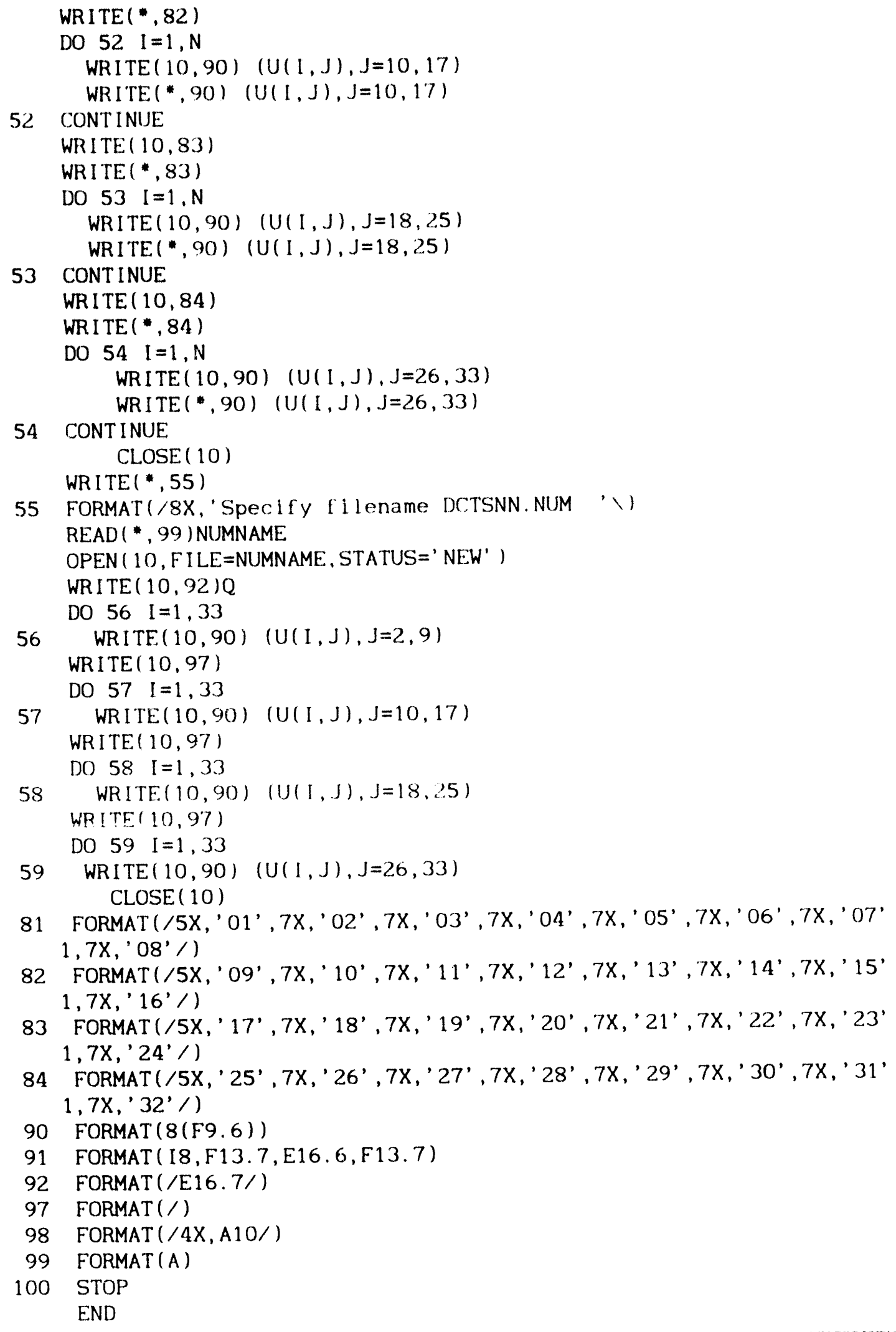


(b) Laminar-Pseudoplast ic Velocity Map. Square Duct

C SQDCT2.FOR VELOCITY MAP, IAMINAR-PSEUDOPLASTIC FLOW IN A SQUARE DUCT C(REQUIRES NEWTONIAN SOLUTION, SQDCTI).

\$NODEBBUG

\$NOFLOATCALLS

\$LARGE

REAL" 8 V $(65,65), U(40,40), F(40,40), \operatorname{VAV}(100), \operatorname{VBER}(100), G F(100)$

CHARAC IER* 16 TRPNAME, DCTNAME, DATNAME, INI TNAME, ARNAME

DATA N, NF, NN, NL, NA, N I , NUM, KAMAX, LCT, DEL/33, 0,65, 1, 1, 1, 1, 48, 1 , $\mathrm{X} 0.015625 /$

$\mathrm{C}-\ldots$

C Norm.: $\quad 0<X, Y<2 a, \quad x=X / 2 a, y=Y / 2 a ; 2 a=$ sidelength; $D E L=\operatorname{de} 1 x=1 / 64$;

C $v=V /\langle V\rangle ; \quad D V W=D E L * d v / d y($ wa 11$) ; \quad Q=D V W / 16 ; \quad P=(2 D V W) * * E M / 16=(32 Q) * * E M / 16$

C Taylor exp: $D V W=[9 v(N, 2)-4.5 v(N, 3)+v(N, 4)] / 3 . \quad(N=N+1$ for relabel).

$C$ Initl ass : prev. calc results for $m=m-0.1$ (start $w$. Newt, $m=1$ )

GF $(N A-1)=0.850$

C (1) CHOOSE FLOW BEHAVIOUR INDEX: $m=1-0.1 \mathrm{k}, \mathrm{k}=1,2, \ldots 5$ WRITE (*, 1$)$

1 FORMAT $\left(/ 8 X,{ }^{\prime} F . B\right.$. Index $m$ for INITL. APPROX (Newt $\left.=1.0\right)::^{\prime} \backslash$ ) READ(* *)EM

C (2) READ IN PREV. CALC AS INITIAL. APPROX., IST QUADRANT WRITE $(*, 50)$

50 FORMAT(/8X. 'Name file to read: DCTnXX.NUM (previous PLSQ]' ') READ (* 99) INITNAME

OPEN (10, FILE=INITNAME, STATUS=' OL.D' )

$\operatorname{READ}(10,92) \mathrm{Q}$

WRITE: $(*, 92) 0$

DO $51 \quad I=1,33$

$\operatorname{READ}(10,90)(1)(1, .3), J=?, 9)$

WRITE (*,90) (U(1,.J),J=?,9)

51 CONTINUE

$\operatorname{READ}(10,97)$

WRITE (*, 97)

DO $52 \quad I=1,33$

$52 \operatorname{READ}(10,90) \quad(U(I, J), J=10,17)$

$\operatorname{READ}(10,97)$

WRITE $(*, 97)$

DO $53 \quad I=1,33$

$53 \operatorname{READ}(10,90) \quad(U(I, J), J=18,25)$

$\operatorname{READ}(10,97)$

WRITE $(*, 97)$

DO $54 \quad I=1,33$

$54 \operatorname{READ}(10,90) \quad(U(I, J), J=26,33)$

CLOSE. ( 10 )

C REVERSE ABSCISSA (MAX TO TOP RICHT) \& RELABEL:

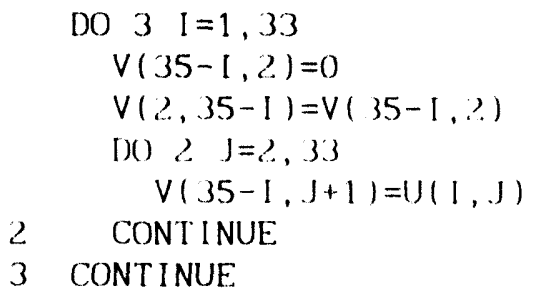




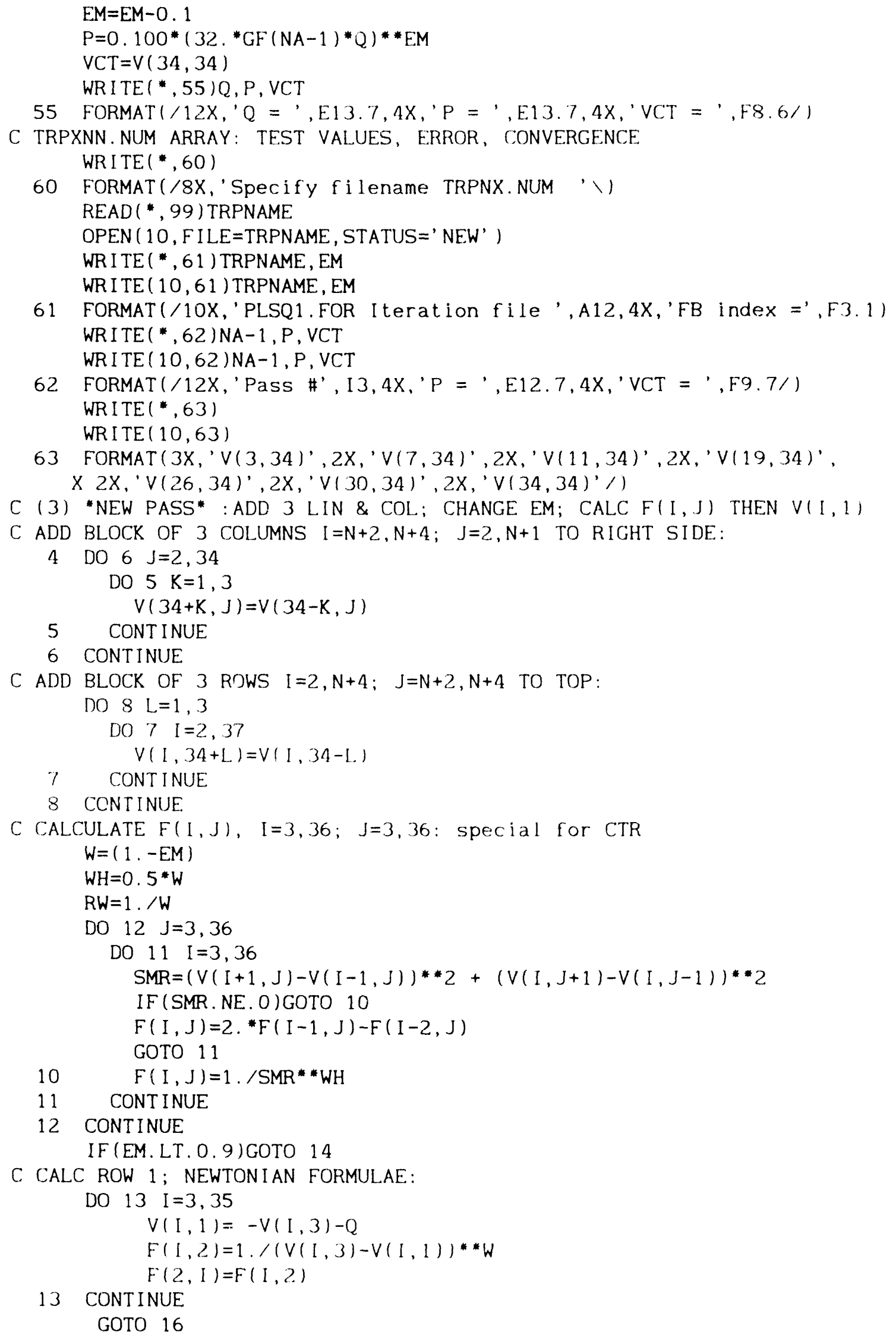


C CALC ROW 1; POWER-LAW FORMULAE:

14 DO $15 \quad I=4,35$

$A 3=V(I+1,3) *(F(I+1,3)+F(I, 3))+V(I-1,3) *(F(I-1,3)+F(I, 3))$

$A 4=V(1,4) *(F(1,4)+F(1,3))$

$B=V(I, 3) *(F(I+1,3)+F(I-1,3)+F(I, 4)+4 * F(I, 3))$

$F(I, 2)=(A 3+A 4-B+P), V(I, 3)$

$F(2,1)=F(1,2)$

$V(I, 1)=V(I, 3)-(V(I, 3) /(A 3+A 4-B+P)) * * R W$

15 CONTINUE

WRITE $(*, 97)$

$\operatorname{WRITE}(10,97)$

ANUM $=V(3,4) *(F(3,4)+F(3,3))-V(3,3) *(F(3,4)+2 . * F(3,3))+P / 2$.

$F(3,2)=$ ANUM $/(3,3)$

$F(2,3)=F(3,2)$

$V(3,1)=V(3,3)-(V(3,3) / A N U M) * R W$

C SET ALL $V(I, 1), V(36,1), F(2,2), F(2,36)$; COPY ROW 1 TO COL.UMN 1:

$16 \quad V(1,1)=0$

$V(2,1)=0$

$V(36,1)=0$

$V(37,1)=0$

$F(2,2)=0$

$F(36,2)=0$

$F(2,36)=0$

DO $17 \quad I=1,37$

$V(1, I)=V(I, 1)$

17 CONTINUE

IF (NA.LE. 1) GOTO 20

C (4) 48 BOUSTROPHEDON FWD-BK, UP-DWN PASSES; THEN SYMMETRIZE QUADRANT

C (DIAG. AVE.); DISPLAY-RECORD 8 SAMPLE (CTRLINE) VALUES EVERY 3D PASS.

18 WRITE $(*, 62) N A-1, P, V C T$

WRITE $(10,62) N A-1, P, V C T$

IF (NF. EQ. 1$)$ GOTO 19

$\mathrm{KAMAX}=48$

GOTO 20

19 KAMAX $=24$

20 DO $29 \mathrm{KA}=1, \mathrm{KAMAX}$

DO $23 \mathrm{~J}=3,35$

DO $21 \quad I=3,35$

$Q I=V(I+1, J) *(F(I+1, J)+F(I, J))+V(I-1, J) *(F(I-1, J)+F(I, J))$

$Q J=V(I, J+1) *(F(I, J+1)+F(I, J))+V(I, J-1) *(F(I, J-1)+F(I, J))$

$D E N=F(I+1, J)+F(I-1, J)+F(I, J+1)+F(I, J-1)+4 . * F(I, J)$

$V(I, J)=(P+Q I+Q J) / D E N$

21 CONTINUE

23 CONTINUE

DO $26 \quad I=3,35$

DO $24 \mathrm{~J}=3,35$

$Q I=V(I+1, J) *(F(I+1, J)+F(I, J))+V(I-1, J) *(F(I-1, J)+F(I, J))$

$Q J=V(I, J+1) *(F(I, J+1)+F(I, J))+V(I, J-1) *(F(I, J-1)+F(I, J))$

$D E N=F(I+1, J)+F(I-1, J)+F(I, J+1)+F(I, J-1)+4 . * F(I, J)$

$V(I, J)=(P+Q T+Q j) / D E N$

24 CONTINUE

26 CONTINUE

DO $28 \quad \mathrm{I}=3,35$

DO $27 \mathrm{~J}=3,35$ 


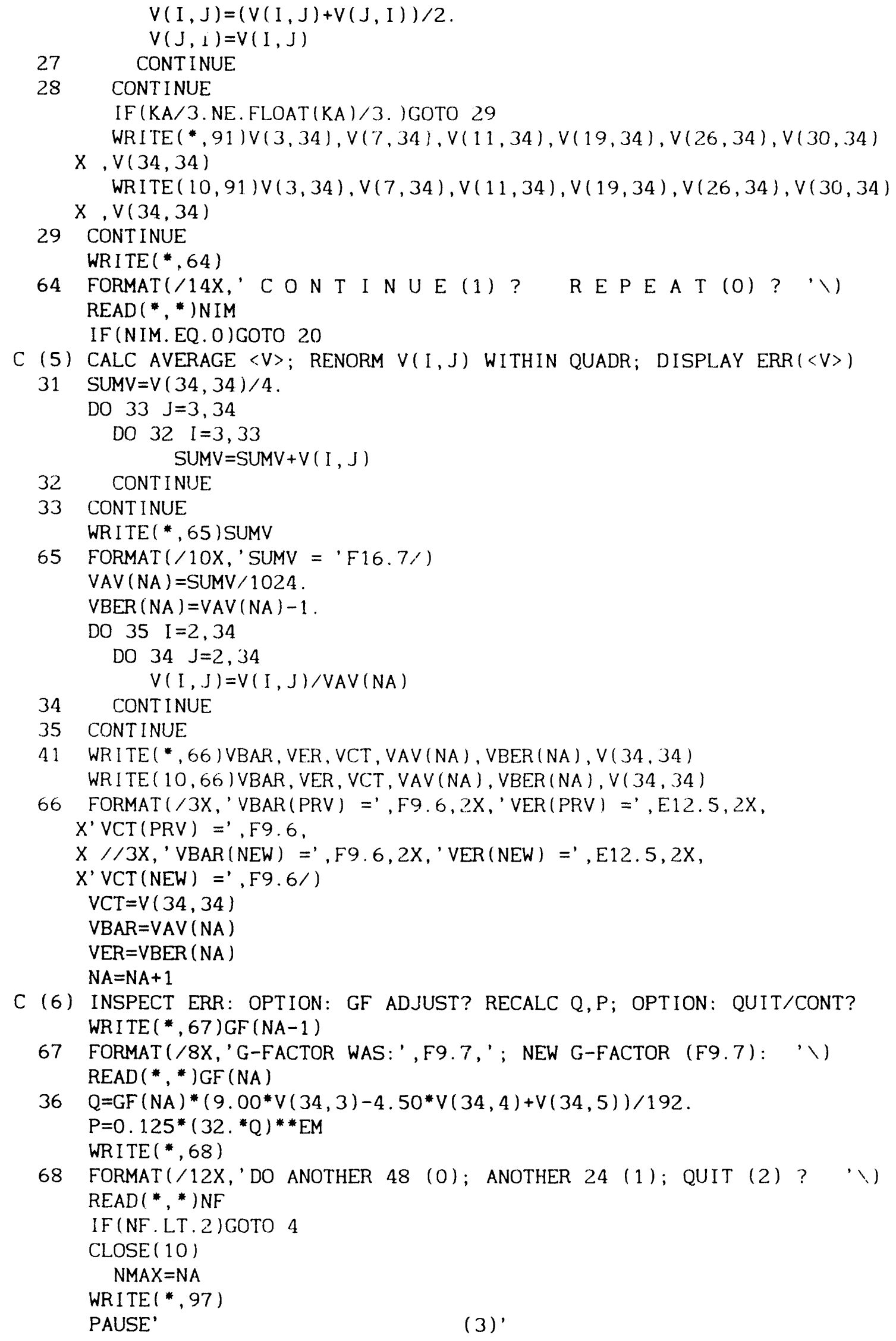

C (5) CALC AVERAGE $\langle V\rangle$; RENORM $V(I, J)$ WITHIN QUADR; DISPLAY ERR $(\langle V\rangle)$

C (6) INSPECT ERR: OPTION: GF ADJUST? RECALC Q,P; OPTION: QUIT/CONT? WRITE $(*, 67) \mathrm{GF}(\mathrm{NA}-1)$

67 FORMAT (/8X, 'G-FACTOR WAS:', F9.7,'; NEW G-FACTOR (F9.7): '\) $\operatorname{READ}(*, *) G F(N A)$

$36 \mathrm{Q}=\mathrm{GF}(\mathrm{NA}) *(9.00 * \mathrm{~V}(34,3)-4.50 * \mathrm{~V}(34,4)+\mathrm{V}(34,5)) / 192$ 
C REVERSE ABSCISSA BEFORE WRITING FILE SUITABLE FOR CONTOUR CALC DO $39 \mathrm{I}=1, \mathrm{~N}$

DO $38 \mathrm{~J}=2, \mathrm{~N}$

$U(I, J)=V(N+2-I, J+1)$

38 CONTINUE

39 CONTINUE

C (7) WRITE INFORMATION AND DAT FILE (FOR CONTOUR PROGRAM)

WRITE $(*, 69)$

69 FORMAT( $/ 8 \mathrm{X}$,' Specify filename DCTsNN. NUM $(\mathrm{s}=1-\mathrm{m}), \backslash)$

READ (*, 99) DATNAME

OPEN ( 10, F ILE=DATNAME, STATUS=' NEW' $)$

WRITE $(10,92) Q$

DO $70 \quad I=1,33$

$70 \quad \operatorname{WRITE}(10,90) \quad(U(I, J), J=2,9)$

WRITE $(10,97)$

DO $71 \quad I=1,33$

71

$\operatorname{WRITE}(10,90) \quad(U(I, J), J=10,17)$

$\operatorname{WRITE}(10,97)$

DO $72 \quad I=1,33$

$72 \operatorname{WRITE}(10,90) \quad(U(I, J), J=18,25)$

WRITE $(10,97)$

DO $73 \quad I=1,33$

$73 \quad \operatorname{WRITE}(10,90) \quad(U(I, J), J=26,33)$

CLOSE (10)

WRITE $(*, 74)$

74 FORMAT(/12X,' Specify filename DCTsNN. INF $(s=1-m)$ ' $\backslash)$

$\operatorname{READ}(*, 99)$ DCTNAME

$\operatorname{OPEN}\left(10\right.$, FILE $=$ DCTNAME, STATUS $={ }^{\prime}$ NEW' $)$

WRITE $(10,98)$ DCTNAME

WRITE $(*, 98)$ DCTNAME

WR I TE $(10,75)$ NMAX, P, Q , VCT

WRITE $(*, 75)$ NMAX, P, Q, VCT

$\operatorname{WRITE}(10,76)$

WRITE $(*, 76)$

DO 40 NA $=1$, NMAX

WRITE $(10,96) N A, \operatorname{VAV}(N A), \operatorname{VBER}(N A), G F(N A)$

WRITE (*, 96$)$ NA, VAV (NA), VBER(NA), GF (NA)

40 CONTINUE

75 FORMAT ( $/ 4 \mathrm{X},{ }^{\prime}$ Pass \#: ', I2, 3X, 'P = ',E12.6,3X, $X^{\prime} \mathrm{Q}={ }^{\prime}, E 12.6,3 X,{ }^{\prime}$ VCT $\left.=', F 9.7 /\right)$

76 FORMAT (/7X, 'N' , 6X, 'V(AVE) ' , 8X, 'ERROR \%' , 8X, 'G-FACTOR' /)

WRITE $(10,97)$

$\operatorname{WRITE}(*, 97)$

WRITE $(10,81)$

WRITE $(*, 81)$

DO $77 \quad I=1, N$

$\operatorname{WRITE}(10,90) \quad(U(I, J), J=2,9)$

WRITE $(*, 90)(U(I, J), J=2,9)$

77 CONTINUE

WRITE $(10,82)$

WRITE(*, 82)

DO $78 \quad I=1, N$

$\operatorname{WRITE}(10,90) \quad(U(I, J), J=10,17)$

$\operatorname{WRITE}(*, 90) \quad(U(I, J), J=10,17)$ 
78 CONTINUE

$\operatorname{WRITE}(10,83)$

$\operatorname{WRITE}(*, 83)$

DO $79 I=1, N$

$\operatorname{WRITE}(10,90) \quad(U(I, J), J=18,25)$

79 CONTINUE

WRITE $(*, 90) \quad(U(I, J), J=18,25)$

WRITE $(10,84)$

WRITE $(*, 84)$

DO $80 I=1, N$

$\operatorname{WRITE}(10,90) \quad(U(I, J), J=26,33)$

WRITE $(*, 90) \quad(U(I, J), J=26,33)$

80 CONTINUE

CLOSE (10)

81 FORMAT (/5X, '01', 7X, '02', 7X, '03', 7X, '04', 7X, '05', 7X, '06', 7X, '07' $\left.1,7 \mathrm{X},{ }^{\prime} 08^{\prime} /\right)$

82 FORMAT(/5X, ' 09', 7X, ' 10', 7X, '11', 7X, '12', 7X, '13', 7X, '14', 7X, '15'

$1,7 X, ' 16^{\prime} / 1$

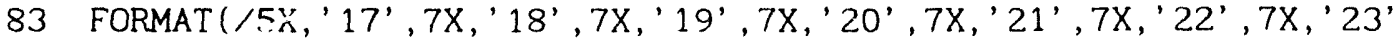

$\left.1,7 \mathrm{X},{ }^{\prime} 24, /\right)$

84 FORMAT(/5X, '25',7X, '26', 7X, '27',7X, '28', 7X, '29', 7X, ' 30', 7X, ' 31'

$\left.1,7 \mathrm{X},{ }^{\prime} 32^{\prime} / 1\right)$

30 FORMAT ( I6)

90 FORMAT ( $8(\mathrm{~F} 9.6))$

91 FORMAT (7(F10.7))

92 FORMAT (/E16.7/)

93 FORMAT $(6(E 12.7))$

94 FORMAT ( I6, I6, E20.8)

95 FORMAT $(2$ I $6,4 \mathrm{E} 15.8)$

96 FORMAT (I8, F13.6,E16.6,F13.5)

97 FORMAT $(/)$

98 FORMAT $(/ 4 X, \mathrm{~A} 10 /)$

99 FORMAT $(\mathrm{A})$

STOP

END

\section{(c) Velocity Contours}

Equal velocity contours are linearly interpolated on the maps produced in the preceding programs. The velocity map is quartered (cut through the centerlines normal to the sides); interpolation continues to the diagonal; the remainder of the contour is obtained by reflection.

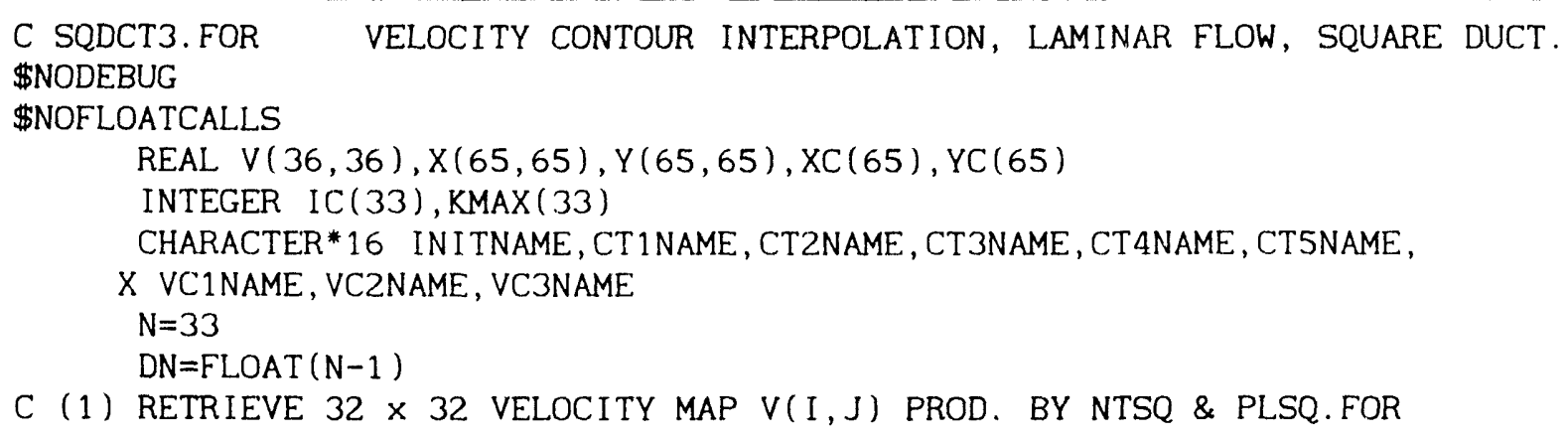

C (1) RETRIEVE $32 \times 32$ VELOCITY MAP V(I,J) PROD. BY NTSQ \& PLSQ. FOR 
WRITE $(*, 50)$

50 FORMAT(/8X,' Name file to read: DCTnXX. NUM ' $\backslash)$

$\operatorname{READ}(*, 99)$ INITNAME

$\operatorname{OPEN}(10$, FILE $=$ INITNAME, STATUS=' OLD' $)$

$\operatorname{READ}(10,92) \mathrm{Q}$

WRITE $(*, 92) Q$

DO $51 \quad I=1,33$

$\operatorname{READ}(10,90) \quad(V(I, J), J=2,9)$

$\operatorname{WRITE}(*, 90) \quad(U(I, J), J=2,9)$

51 CONTINUE

$\operatorname{READ}(10,97)$

WRITE $(*, 97)$

DO $52 \quad I=1,33$

$52 \operatorname{READ}(10,90) \quad(\mathrm{V}(\mathrm{I}, \mathrm{J}), \mathrm{J}=10,17)$

$\operatorname{READ}(10,97)$

WRITE $(*, 97)$

DO $53 \quad I=1,33$

$53 \operatorname{READ}(10,90) \quad(\mathrm{V}(\mathrm{I}, \mathrm{J}), \mathrm{J}=18,25)$

$\operatorname{READ}(10,97)$

WRITE $(*, 97)$

DO $54 \quad I=1,33$

$\operatorname{READ}(10,90) \quad(V(I, J), J=26,33)$

$\operatorname{WRITE}(*, 90) \quad(V(I, J), J=26,33)$

54 CONTINUE

CLOSE (10)

C (2) CALC CONTOURS AT 20 EQUAL VELOC. INTERVALS BY LIN. INTPOL.

WRITE $(*, 55)$

55 FORMAT (/8X,' Skip stops between contours ? (1) ' $\backslash)$

$\operatorname{READ}(*, *) N O$

$M=0$

$\mathrm{VCT}=\mathrm{V}(1,33)$

$3 \quad M=M+1$

VREF $=0.05 *$ VCT $*$ FLOAT $(M)$

IF (NO.EQ. 1$)$ GOTO 56

WRITE $(*, 97)$

PAUSE' Start NEW velocity contour: VREF $=0.05^{*} M^{*} V C T, M=1 \ldots 19^{\prime}$

$C$ INCREASE $X$ STEPWISE; LINEAR INTERPOL FOR $Y$, TO 45 DEG ( $I=J)$ CORNER

56 WRITE $(*, 97)$

DO $4 \mathrm{~J}=2,33$

$\operatorname{IF}(V(1, J)$. GE. VREF $)$ GOTO 5

4 CONTINUE

5 DO $7 \quad \mathrm{I}=1,32$

AJS $=$ FLOAT $(. J-2)$

IF (I.EQ. 35-J)GOTO 8

IF (VREF.LT.V $(I, J))$ GOTO 6

$\mathrm{J}=\mathrm{J}+1$

AJS $=$ FLOAT $(J-2)$

IF ( I.EQ. 35-J)GOTO 8

$6 \quad Y(I, M)=(A J S+(V R E F-V(I, J-1)) /(V(I, J)-V(I, J-1))) / D N$

$X(I, M)=F L O A T(I-1) / D N$

WRITE $(*, 91) M, J-2,35-J, I, X(I, M), Y(I, M)$

7 CONTINUE

C CORNER: CALC CORNER $X, Y$ \& COPY REMAINING NUMBER OF POINTS (MIRR IMGE)

$8 \quad I C(M)=I$ 


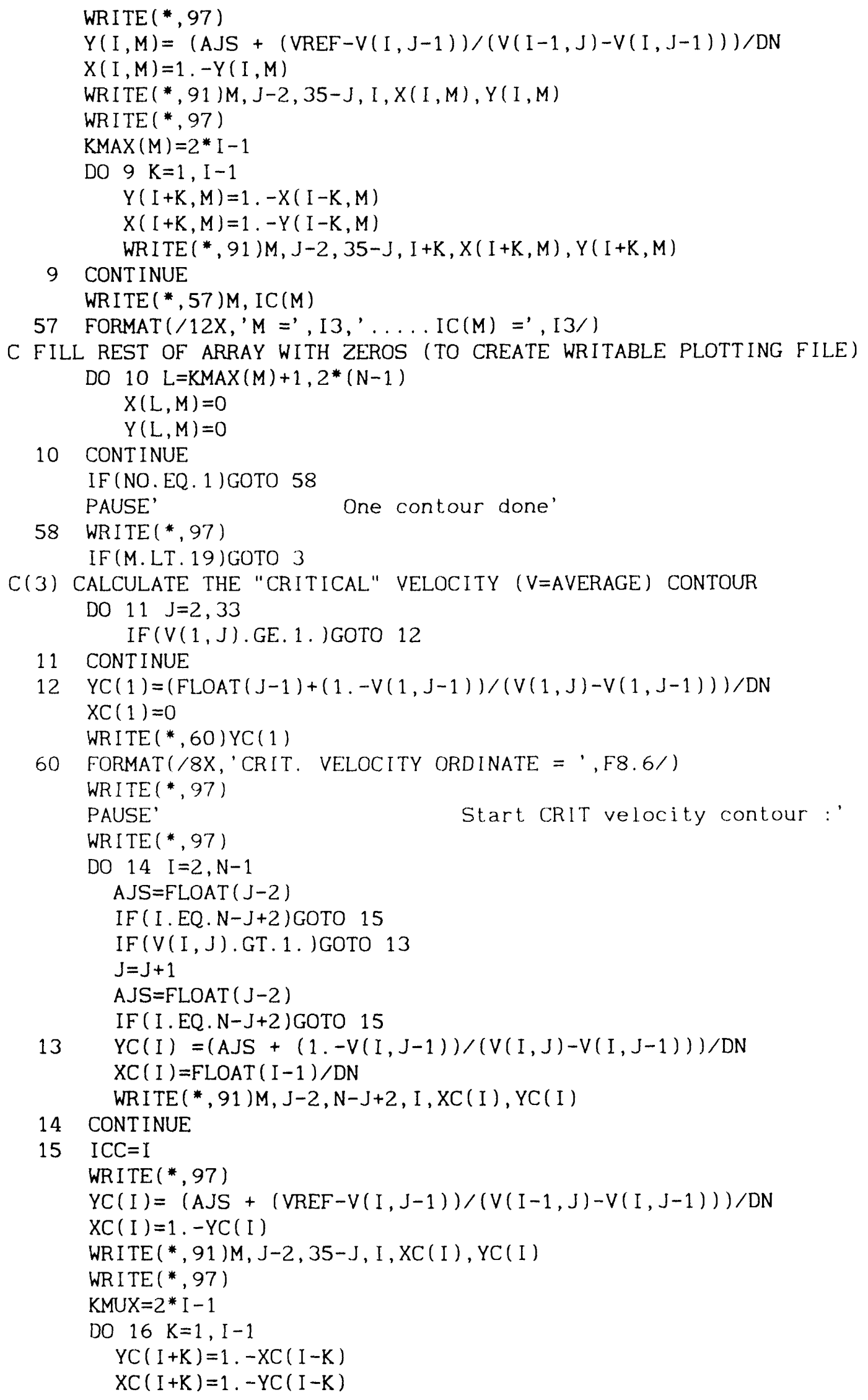




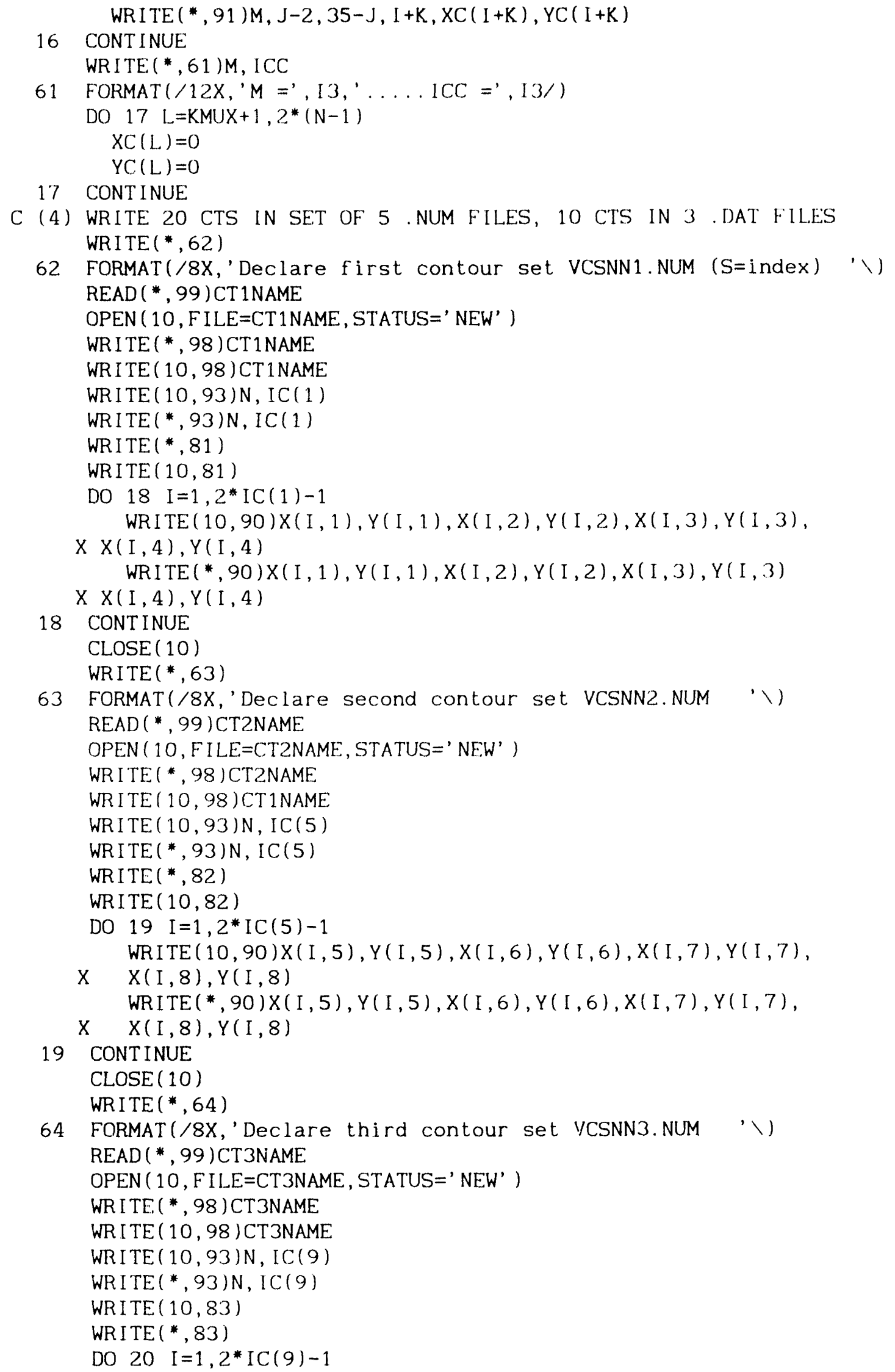




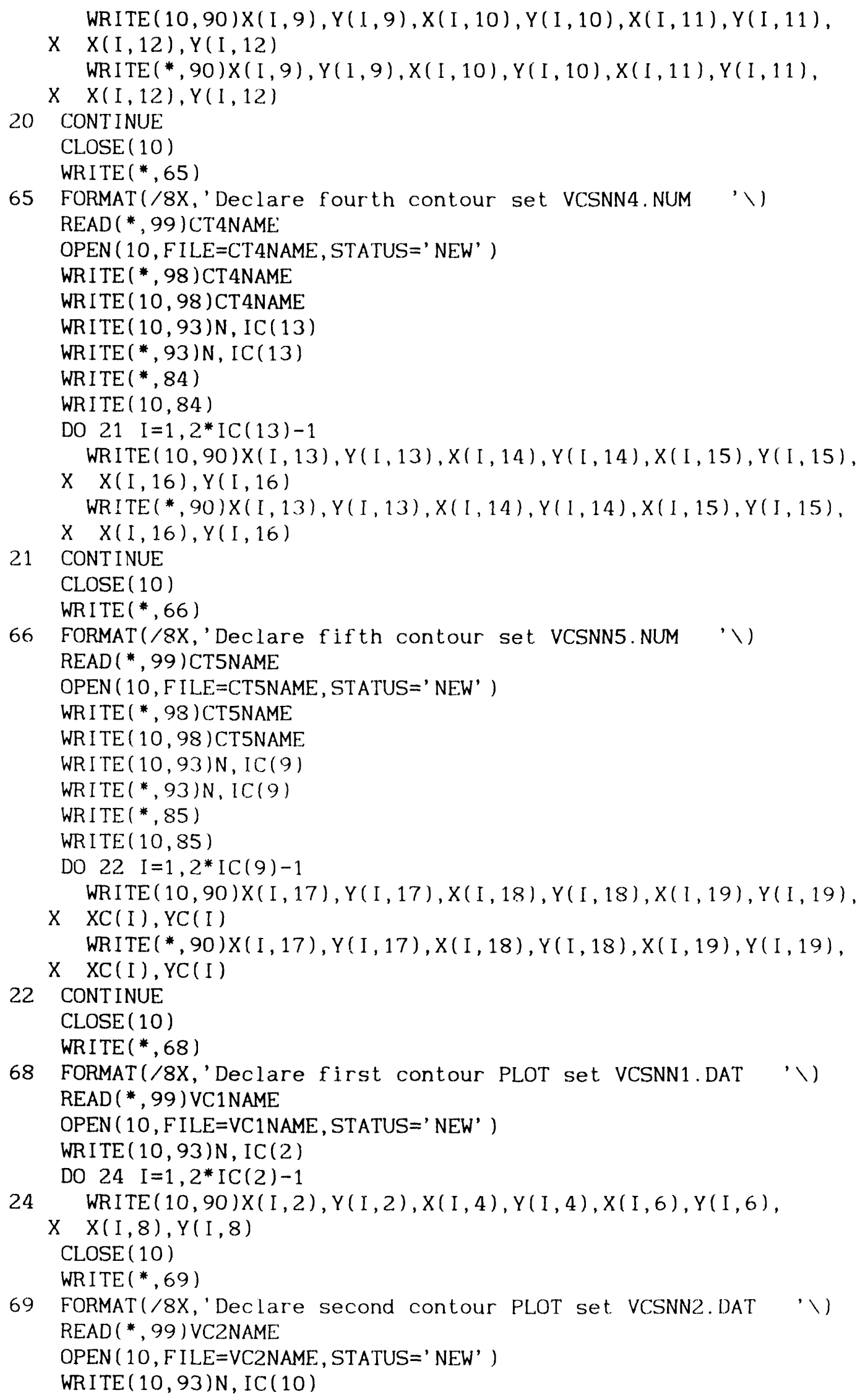




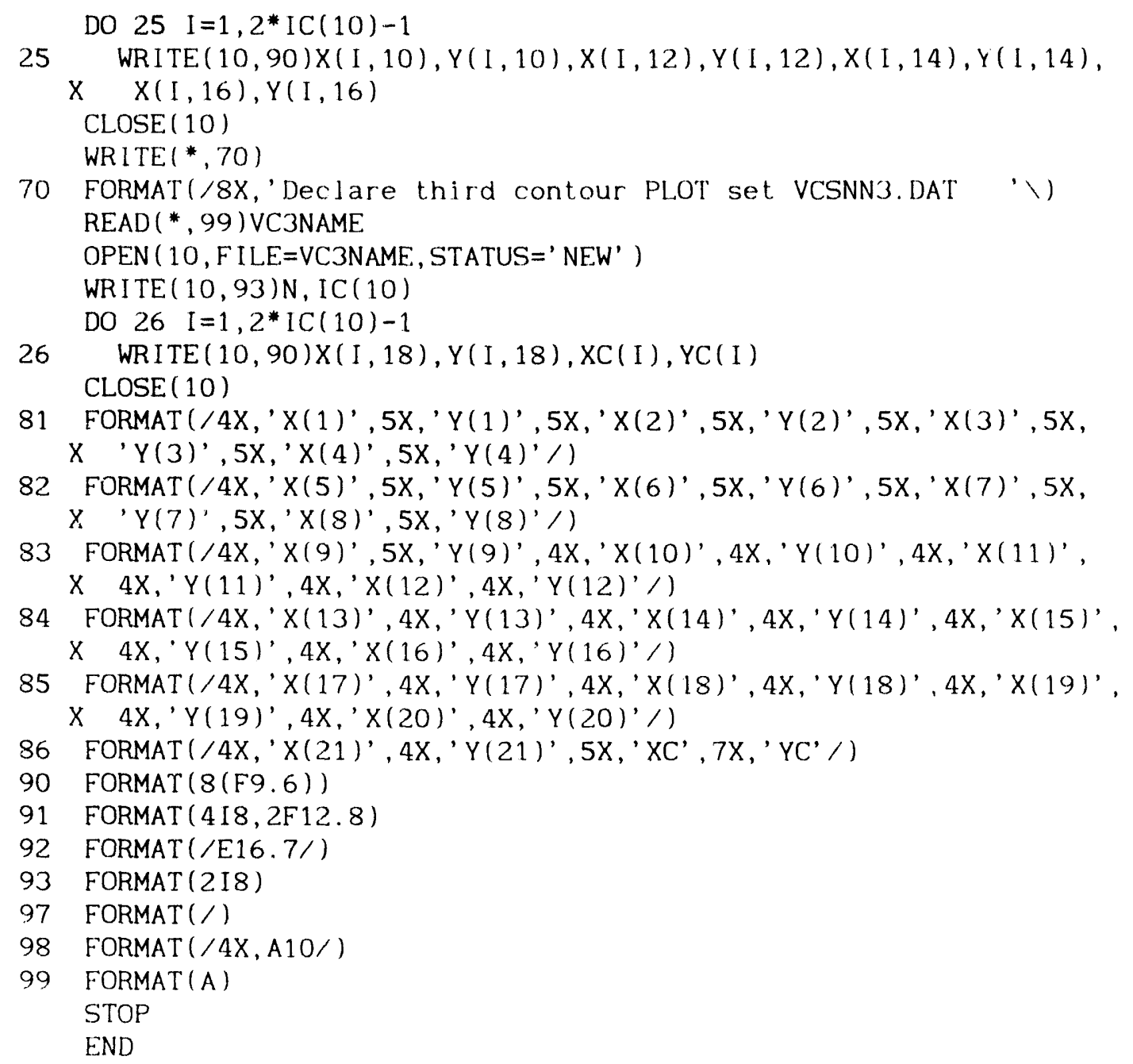

(II) EQUIPOTENTI ALS IN CIRCULAR-AXIAL GEOMETRY

Equipotentials and field lines for circular segment electrode geometries that feature at least one reflection symmetry plane (such as "Wrapped-Plate" schemes, widely used in EM Field sensing) can be conveniently calculated and plotted by solving the Poisson Equation after a Schwartz-Christoffel transform to Cartesian geometry. Typically, these schemes further provide an Outer Envelope that serves as an electric shield, dust cover, mechanical support etc. One of the advantages of the Schwartz transform is that regions of different. dielectric constant that consist of annular segments are transformed into rectangles, whence electric Field and Displacement continuity equations become derivatives with respect to the Cartesian coordinates.

For purposes of this discussion, let the symmetry plane between electrodes be labeled A and the symmetry plane that cuts electrodes centrally be labeled B. The electrodes can be driven so as to produce a potential distribution that is reflection-symmetric with respect to plane A. However, it is more practical to drive one electrode and sense the opposite electrode, at AC ground. The outer envelope thus is at the AC Signal Electrode level 0, the "Driver" Electrode at $-V$ and the symmetry plane at $-V / 2$ (for mapping, it is convenient to 
let Signal ( $\&$ Outer Envelope) potential $=+1$, Driver at $=-1$ and Origin at 0 ). In applying the Schwartz conformal transform,

$$
\begin{array}{ll}
r+j \vartheta=\exp (y+j x), & (B-1) \\
y=\log (r), x=N \vartheta / \pi, & (B-2)
\end{array}
$$

boundary conditions turn out to be something of a problem: first, the logarithm of the radius (= Cartesian ordinate) goes to - $\infty$ at the origin $(V=0)$; secondly, the potential along any radial cut connecting the duct surface and outer envelope is not known, yet is needed since one half of the duct, cut anywhere (but, preferably, along either of the two symmetry planes) must be transformed at a time. The difficulty at the origin can be coped with by choosing $V=0$ at $y=-50$, say, a small but finite radius. The potential between inner (duct) and outer (envelope) radius, $r(D)$ and $r(E)$, can be approximated by

$$
V=\log [r / r(D)] / \log [r(E) / r(D)] \quad(B-3)
$$

for the cut along plane A. An initial approximation map can be produced very simply, by linear approximation between boundary potential values. Iteration,

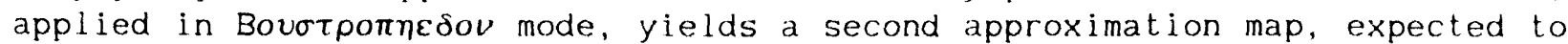
be reasonably accurate in those regions that are furthest away from the cut where approximation (B-3) was used. The initial approximation routine is repeated for the opposite half-duct. These two maps are then combined and rearranged, producing maps cut at plan, B. Iteration is applied again, thus producing a better potential distribution than the approximation (B-3). In this manner, alternate maps cut along A \& B yield an accurate map in a few repeats.

This map is now used to produce sets of equipotentials by 1 inear interpolation, first for the signal side and then for the driver side of the duct; a zero equipotential is interpolated by another short routine and all these files are converted to circular geometry:

$$
\begin{array}{ll}
x^{\prime}=-\exp (y) \cos (j x / \pi n) & (B-4 a) \\
y^{\prime}=\exp (y) \sin (j x / \pi n) & (B-4 b)
\end{array}
$$

for $\mathrm{n}$ mesh points.

Excerpts from these programs are reproduced below. To save space, routine details like Formatting, Instructions for writing, DIMENSION Statements etc. have been omitted.

C WP1E1.FOR: WRAPPED PLATE (w/o guard). $P($ Sig) $=1, P(D r i v e)=-1$ at $r=20$; C $P($ surrd $)=1$ at $r=40 ; P=0$ at $y=\log (E-10)$ : Cartes. grid ( $x=$ angle (rad)).

(Statements omitted)

DATA IZ, IA , IB, IC, IM, JZ, JH, JM, PI , A, Y (1), R( 1$) / 1,6,56,31,61,1,21,41$,

X 3.141592654,0.05235988,-50.,1.9287498E-22/

(Setup: Unifm. Dielectric; Outer envelope $(r=40)$ at SIG potent1.; 
Electrodes $(r=20)$ cover $150 \mathrm{deg}$. , mesh Interval $(1)=3 \mathrm{deg}$.

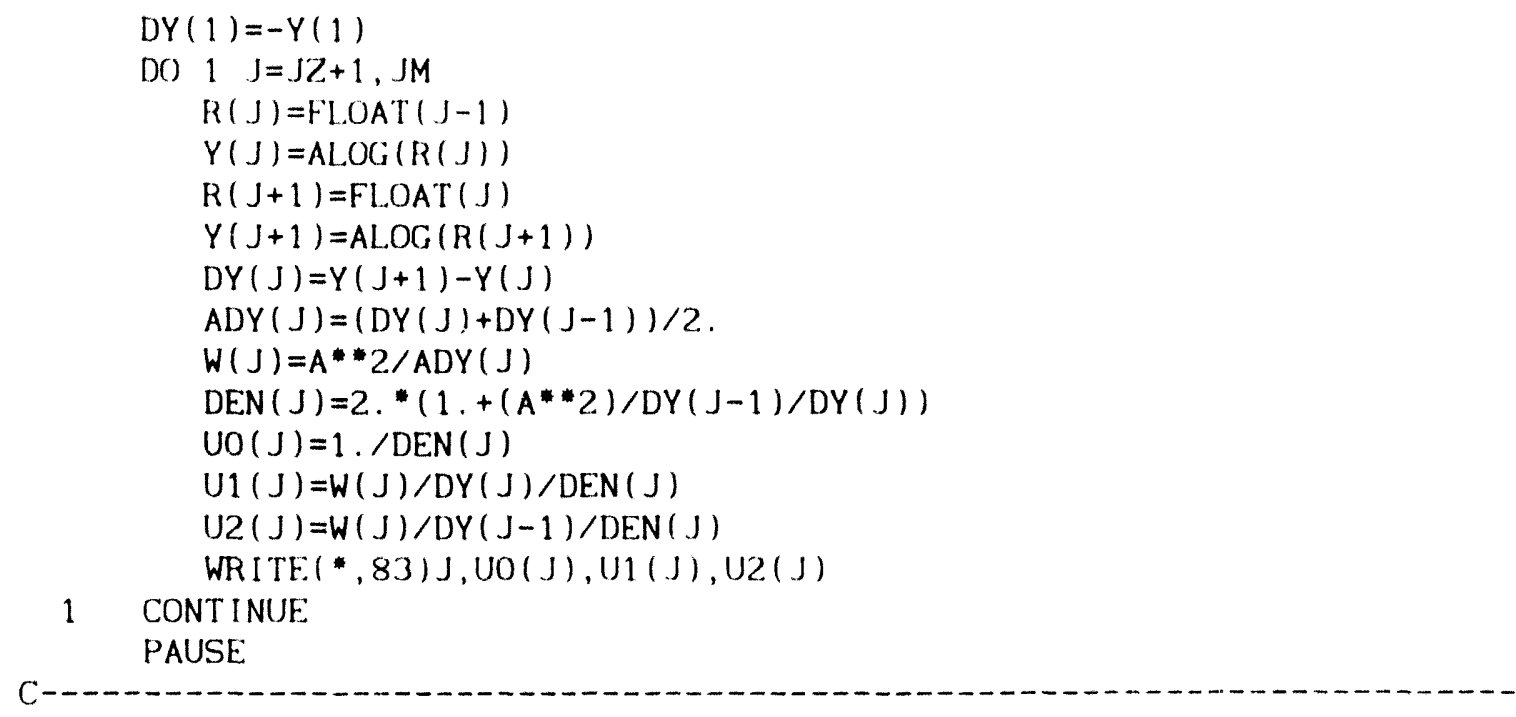

(Write option omitted)

C2 SET FLAG MP=2 ( $S I G), M P=0$ (DRIV), $S=F L O A T(M P-1)=+1(S),-1(D)$

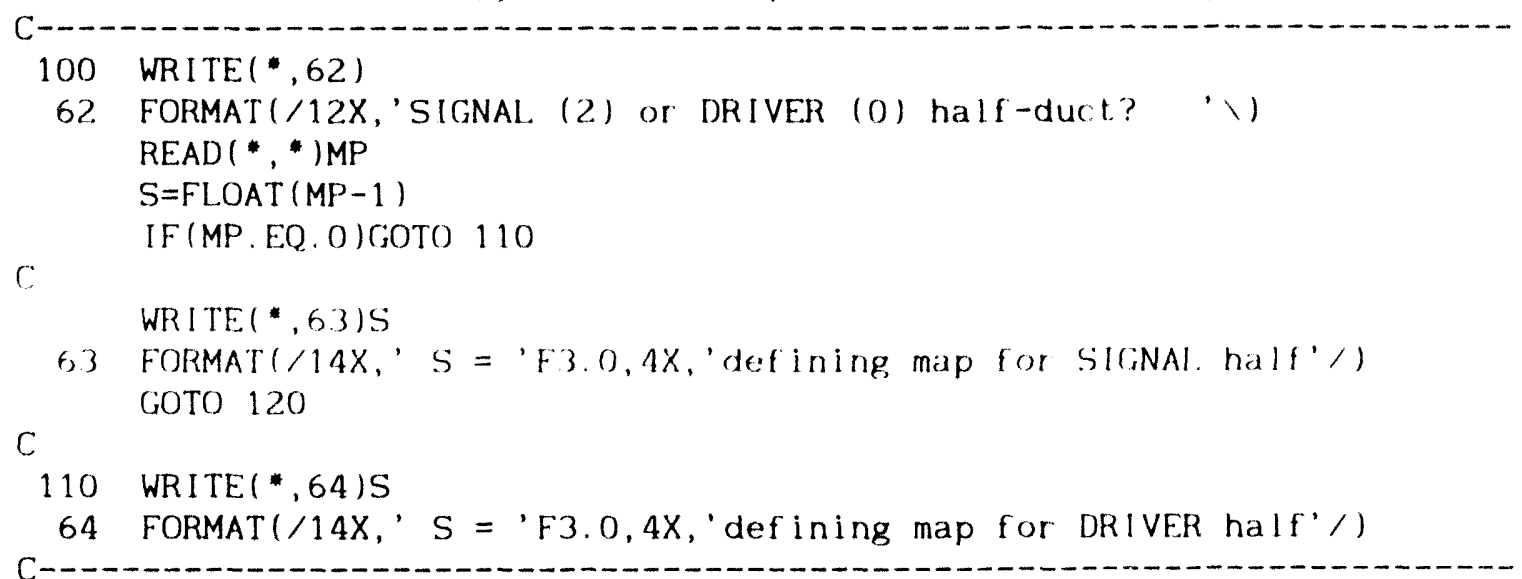

C3 DEFINE $P(I, J)$ ON BOUNDARIES (LEFT \& RIGHT, TOP \& BOTTOM, ELECTRODE):

C (1) BOTTOM \& TOP (ZERO, +1) - 120

DO $121 I=I Z$, IM

$P(I, J Z)=0$

$P(I, J M)=1$.

121 CONTINUE

DO $122 \quad I=I A, I B$

$\mathrm{P}(\mathrm{I}, J \mathrm{H})=\mathrm{S}$

122 CONTINUE

DO $123 \mathrm{~J}=\mathrm{JZ}+1, \mathrm{JH}$

$P(I Z, J)=0$

$P(I M, J)=P(I Z, J)$

123 CONTINUE.

DO $124 \mathrm{~J}=\mathrm{JH}+1, \mathrm{JM}-1$

$\operatorname{ART}(J)=$ FLOAT $(J-1) / F L O A T(J H-1)$

$\mathrm{P}(\mathrm{IZ}, J)=\operatorname{ALOG}(\operatorname{ART}(J)) / \operatorname{ALOG}(2$.

$P(I M, J)=P(I Z, J)$ 


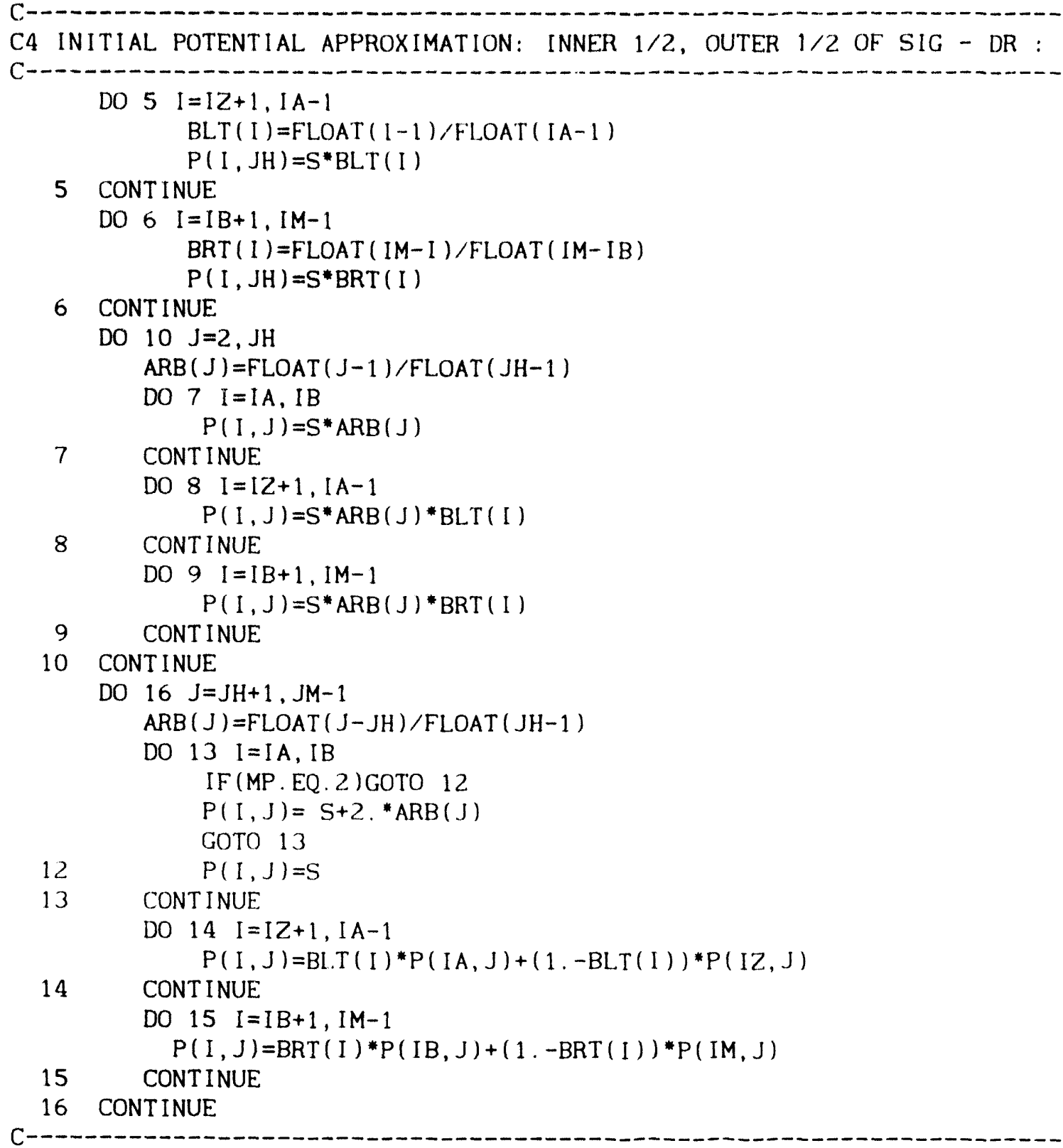

C5 WRITE/DISPLAY OPTION: INITIAL POTENTIAL MAP APPROXIMATION : (omitted)

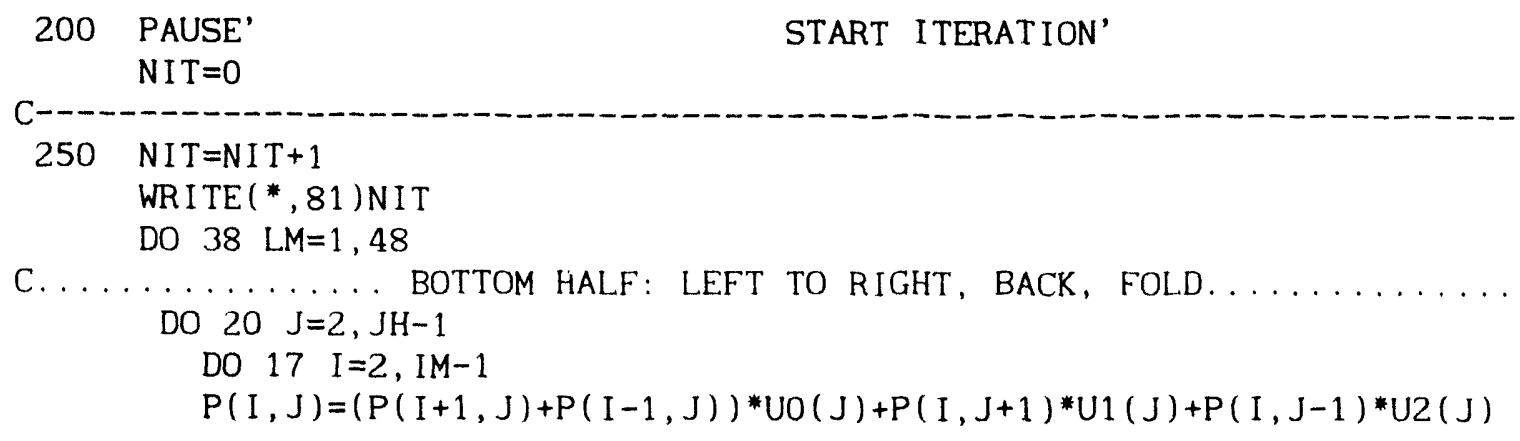

C........... BOTTOM HALF: LEFT TO RIGHT, BACK, FOLD......... DO $20 \mathrm{~J}=2, \mathrm{JH}-1$ DO $17 \mathrm{I}=2, \mathrm{IM}-1$ $P(I, J)=(P(I+1, J)+P(I-1, J)) * U 0(J)+P(I, J+1) * U 1(J)+P(I, J-1) * U 2(J)$ 
DO $18 K=2, I M-2$

$\mathrm{I}=\mathrm{IM}-\mathrm{K}$

$P(I, J)=(P(I+1, J)+P(I-1, J)) * U O(J)+P(I, J+1) * U 1(J)+P(I, J-1) * U 2(J)$

18 CONTINUE

DO $19 \quad I=2, I C-1$

$P(I, J)=(P(I, J)+P(I M+1-I, J)) / 2$.

$P(I M+1-I, J)=P(I, J)$

19 CONTINUE

20 CONTINUE

C........ SIDES ALONG SIGNAL ELECTRODE $J=J H$ : LEFT TO RIGHT, BACK..... $\mathrm{J}=\mathrm{JH}$

DO $21 I=I Z+1, I A-1$

$P(I, J)=(P(I+1, J)+P(I-1, J)) * U O(J)+P(I, J+1) * U 1(J)+P(I, J-1) * U 2(J)$

21 CONTINUE

DO $22 K=2, I A-I Z-1$

$\mathrm{I}=\mathrm{I} A-\mathrm{K}$

$P(I, J)=(P(I+1, J)+P(I-1, J)) * U O(J)+P(I, J+I) * U 1(J)+P(I, J-1) * U 2(J)$

22 CONTINUE

DO $23 \mathrm{I}=\mathrm{IB}+1, \mathrm{IM}-1$

$P(I, J)=(P(I+1, J)+P(I-1, J)) * U 0(J)+P(I, J+1) * U 1(J)+P(I, J-1) * U 2(J)$

23 CONTINUE

DO $24 K=2, I M-I B-1$

$\mathrm{I}=\mathrm{IM}-\mathrm{K}$

$P(I, J)=(P(I+1, J)+P(I-1, J)) * U 0(J)+P(I, J+1) * U 1(J)+P(I, J-1) * U 2(J)$

24 CONTINUE

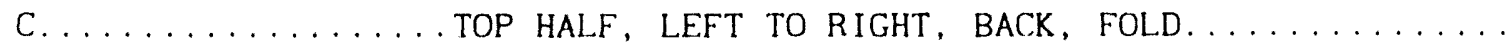

DO $28 \mathrm{~J}=\mathrm{JH}+1, \mathrm{JM}-1$

DO $25 \mathrm{I}=2, I M-1$

$P(I, J)=(P(I+1, J)+P(I-1, J)) * U O(J)+P(I, J+1) * U 1(J)+P(I, J-1) * U 2(J)$

25 CONTINUE

DO $26 K=2, I M-2$

$\mathrm{I}=\mathrm{IM}-\mathrm{K}$

$P(I, J)=(P(I+1, J)+P(I-1, J)) * U 0(J)+P(I, J+1) * U 1(J)+P(I, J-1) * U 2(J)$

26 CONTINUE

DO 27 I $=2, I C-1$

$P(I, J)=(P(I, J)+P(I M+1-I, J)) / 2$.

$P(I M+1-I, J)=P(I, J)$

27 CONTINUE

28 CONTINUE

C........... VERTICAL UP, DOWN; LEFT SIDE - RIGHT SIDE........ DO $32 I=I Z+1, I A-1$

DO $30 \mathrm{~J}=2, \mathrm{JM}-1 \mathrm{e}$

30 CONTINUE

DO $31 \mathrm{~L}=2, \mathrm{JM}-2$

$\mathrm{J}=\mathrm{JM}-\mathrm{L}$

$P(I, J)=(P(I+1, J)+P(I-1, J)) * U 0(J)+P(I, J+1) * U 1(J)+P(I, J-1) * U 2(J)$

31 CONTINUE

32. CONTINUE

DO $35 \mathrm{I}=\mathrm{IB}+1, \mathrm{IM}-1$

DO $33 \mathrm{~J}=2, \mathrm{JM}-1$

33

$P(I, J)=(P(I+1, J)+P(I-1, J)) * U O(J)+P(I, J+1) * U 1(J)+P(I, J-1) * U 2(J)$ CONTINUE 


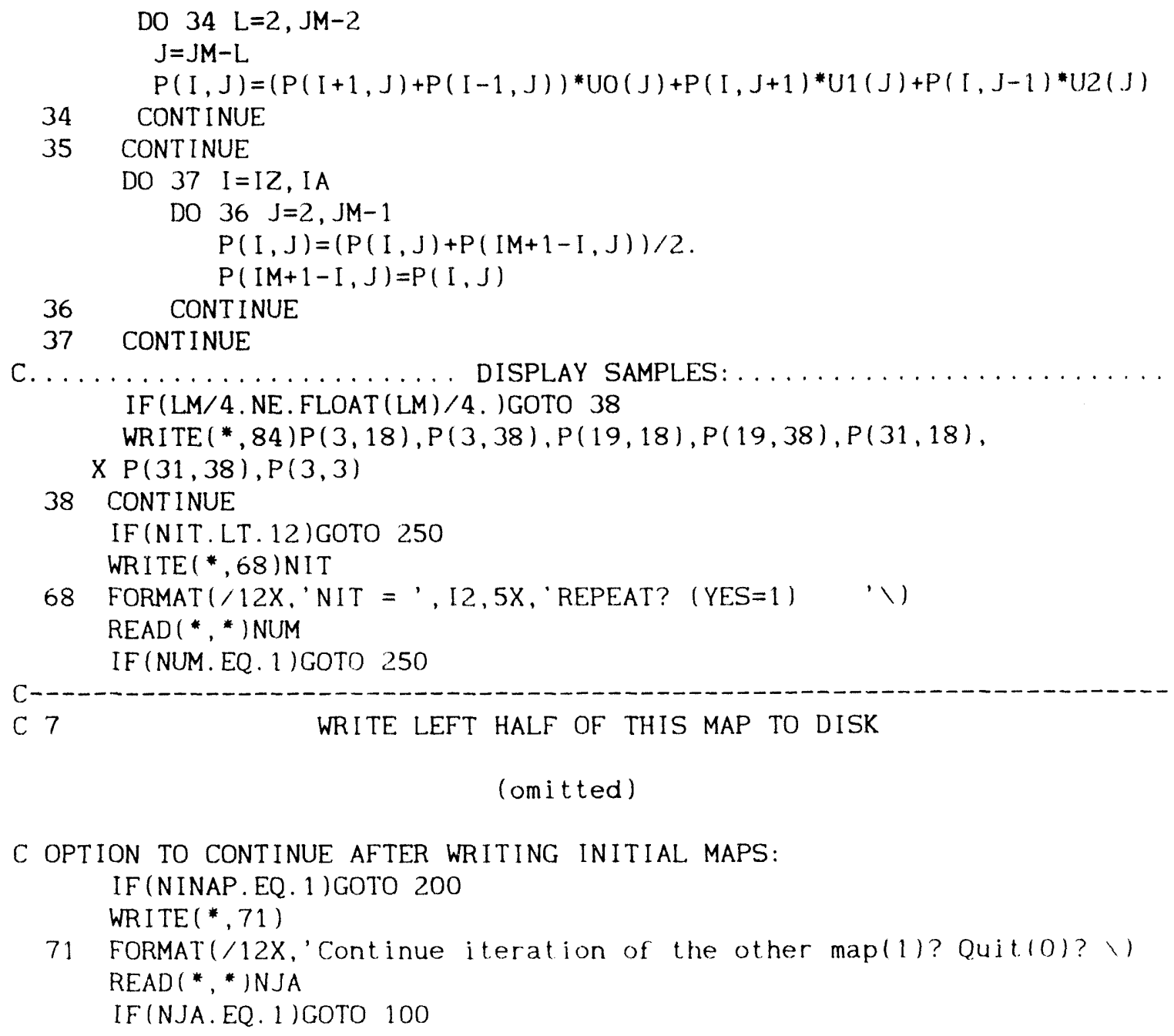

C WP1E2.FOR: WRAPPED PLATE without guard, asymmetric drive 09/18/92 C Follow-up to WP1D1.FOR, varying CUT across/between electrodes for reC calculation of initially assumed CUT boundary values. REPEAT option.

\section{(Initial Statements omitted)}

DATA PI , A, Y ( 1 ) , DY ( 1 ), IS, I I , IZ, IA, IC, IB, IM, JS, JZ, JH, JM, NN, NNA/ X 3.141592654, 0.05235988, $-50 ., 50 ., 5,1,6,31,56,61,20,1,21,41,1,0$ /

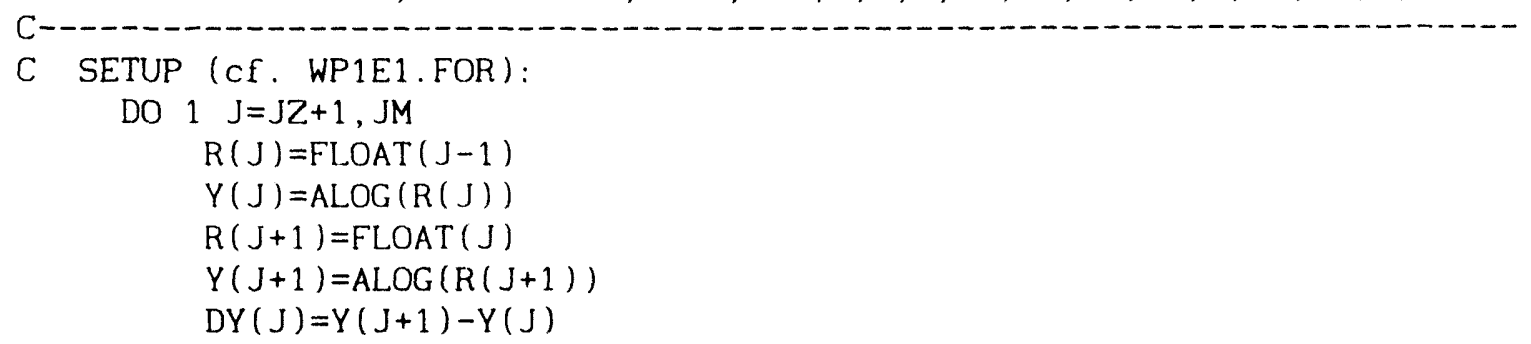




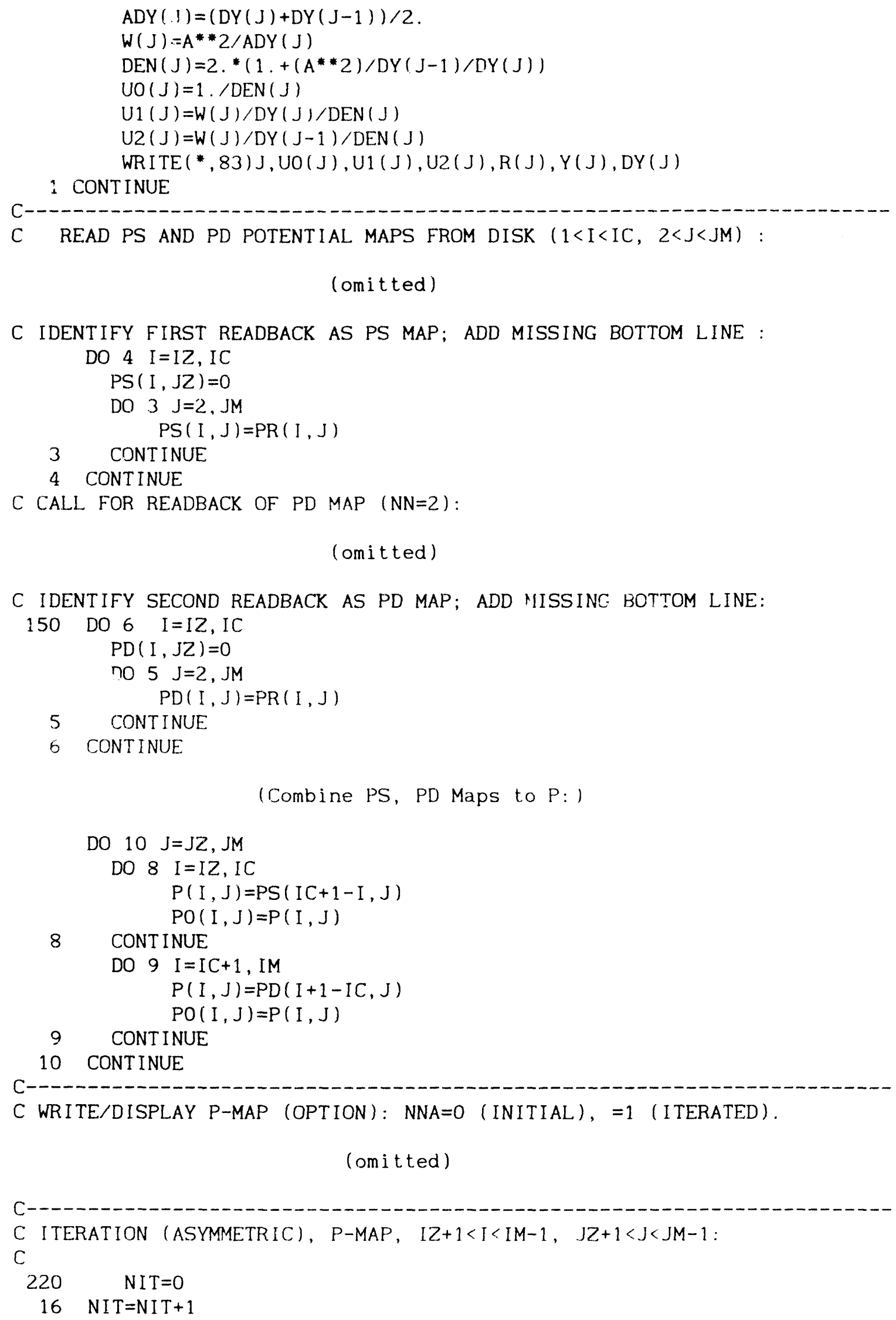


WRITE $(*, 81)$ NIT

DO 28 LM $=1,48$

C BOTTOM HALF, FULL WIDTH, FWD \& BACK:

DO $19 \mathrm{~J}=\mathrm{JZ}+1, \mathrm{JH}-1$

DO $17 \quad I=2, I M-1$

17 CONTINUE

DO $18 \mathrm{~K}=2, \mathrm{IM}-2$

$\mathrm{I}=\mathrm{IM}-\mathrm{K}$

$P(I, J)=(P(I+1, J)+P(I-1, J)) * U D(J)+P(I, J+1) * U 1(J)+P(I, J-1) * U 2(J)$

18 CONTINUE

19 CONTINUE

C ELECTRODE LINE, FWD \& BACK:

$\mathrm{J}=\mathrm{JH}$

DO $20 I=I C-I I+1, I C+I I-1$

20 CONTINUE

DO $2.1 K=2,2^{*} I I-1$

$\mathrm{I}=\mathrm{IC}+\mathrm{I} \mathrm{I}-\mathrm{K}$

$P(I, J)=(P(I+1, J)+P(I-1, J)) * U 0(J)+P(I, J+1) * U 1(J)+P(I, J-1) * U 2(J)$

21 CONTINUE

C TOP HALF, FULL WIDTH; FWD \& BACK:

DO $24 \mathrm{~J}=\mathrm{JH}+1, \mathrm{JM}-1$

DO $22 I=2, I M-1$

$P(I, J)=(P(I+1, J)+P(I-1, J)) * U O(J)+P(I, J+1) * U 1(J)+P(I, J-1) * U 2(J)$

22 CONTINUE

DO $23 K=2, I M-2$

$I=I M-K$

$P(I, J)=(P(I+1, J)+P(I-1, J)) * U 0(J)+P(I, J+1) * U 1(J)+P(I, J-1) * U 2(J)$

23 CONTINUE

24 CONTINUE

C CENTRAL STRIP, UP \& DOWN ALL THE WAY:

DO 27 I = IC $-I I+1, I C+I I-1$

DO $25 \mathrm{~J}=\mathrm{JZ}+1, \mathrm{JM}-1$

$P(I, J)=(P(I+1, J)+P(I-1, J)) * U 0(J)+P(I, J+1) * U 1(J)+P(I, J-1) * U 2(J)$

25 CONTINUE

DO $26 \mathrm{~L}=2, \mathrm{JM}-2$

$J=J M-L$

$P(I, J)=(P(I+1, J)+P(I-1, J)) * U O(J)+P(I, J+1) * U 1(J)+P(I, J-1) * U 2(J)$

26 CONTINUE

27 CONTINUE

C-1-

C DISPLAY SAMPLES OF P AND PO MAPS: STOP WHEN ITERATION CONVERGES ...

IF (LM/4. NE. FLOAT (LM)/4.) GOTO 28

WRITE $(*, 84) P(31,22), P(31,27), P(31,32), P(31,37), P(26,30)$,

$X \quad P(36,20), P(40,20)$

28 CONTINUE

IF (NIT.LT. 6)GOTO 16

WRITE $(*, 97)$

WRITE $(*, 84) P O(31,22), P O(31,27), P O(31,32), P O(31,37), P O(26,30)$,

$X \quad P O(36,20), P O(40,20)$

WRITE $(*, 64)$ NIT

64 FORMAT (/16X,'NIT = ', I2,5X, 'REPEAT ? (1) ? ’ 
$\operatorname{READ}(*, *) N 1$

IF (N1.EQ. 1$)$ GOTO 16

C WRITE P-MAP TO DISC:

(omitted)

(Recombine P-MAP parts for Initial Cut Q-MAP:)

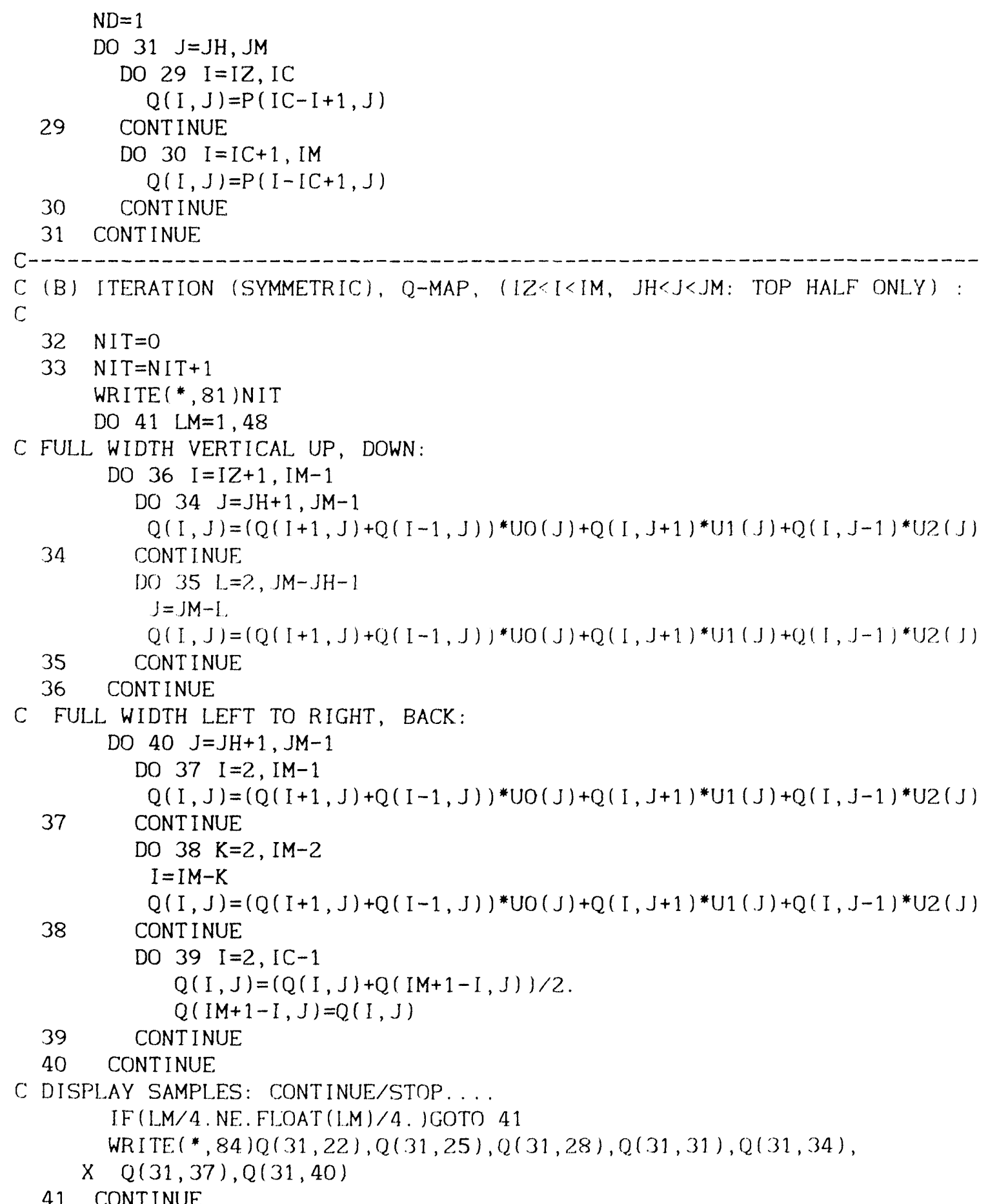

41 CONTINUE 
IF (NIT. LT . 4)GOTO 33

C WRITE STARTING VALUES FOR COMPARISON:

WRITE $(*, 97)$

IF (ND.EQ. 2 )GOTO 42

WRITE $(*, 84) \operatorname{PS}(31,22), \operatorname{PS}(31,25), \operatorname{PS}(31,28), \operatorname{PS}(31,31), \operatorname{PS}(31,34)$,

$X \operatorname{PS}(31,37), \operatorname{PS}(31,40)$

C GOTO 43

$42 \operatorname{WRITE}(*, 84) \operatorname{PD}(31,22), \operatorname{PD}(31,25), \operatorname{PD}(31,28), \operatorname{PD}(31,31), \operatorname{PD}(31,34)$, $X \operatorname{PD}(31,37), \operatorname{PD}(31,40)$

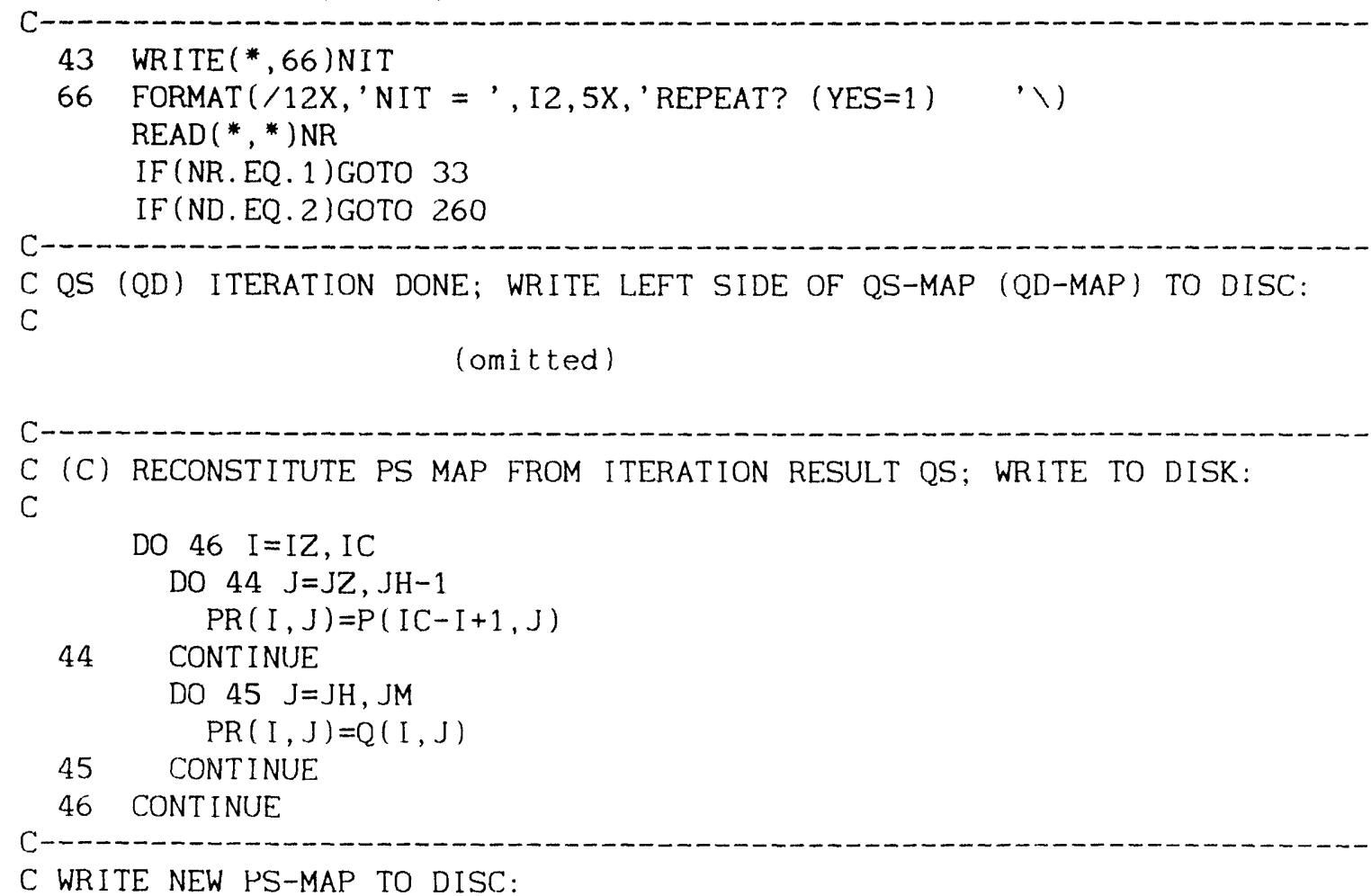
(omitted)

C REARR. P-MAP TO QD-MAP (ITERATE; REARRANGE QS AS PD, WRITE; QUIT

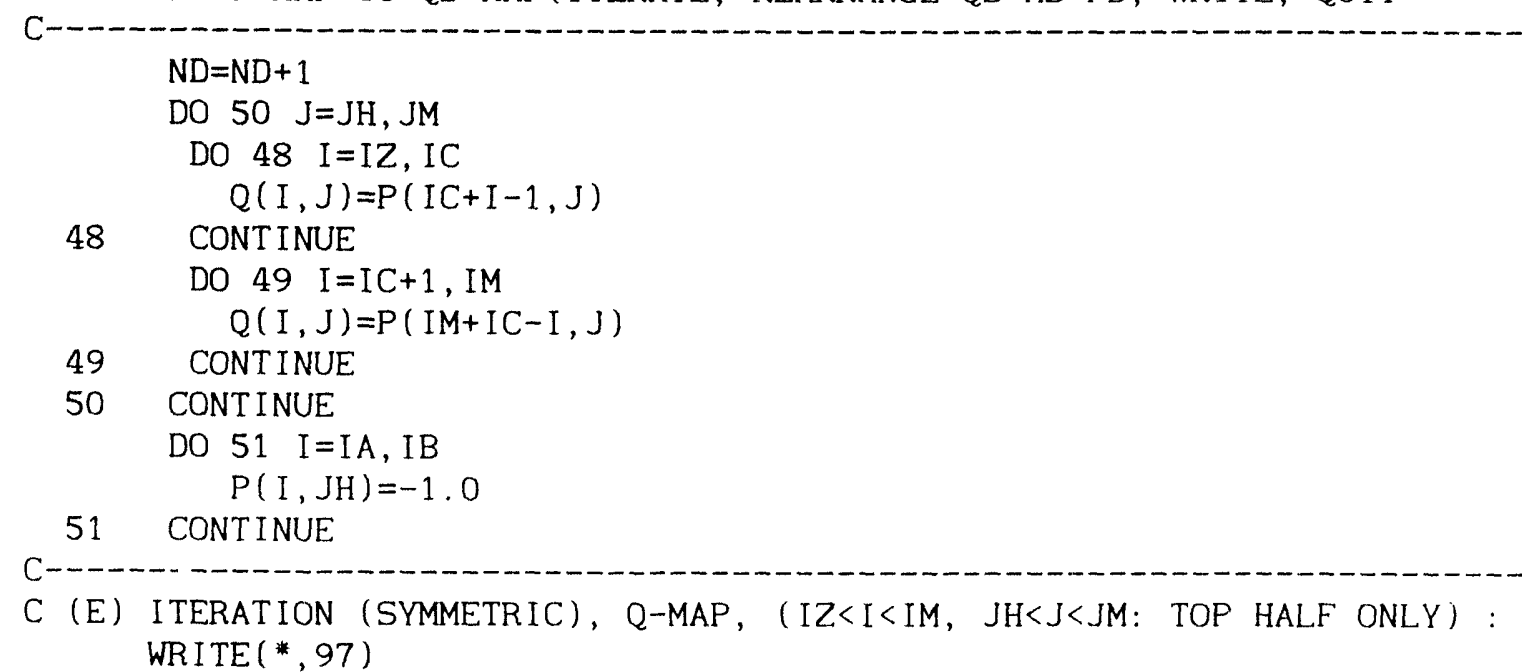




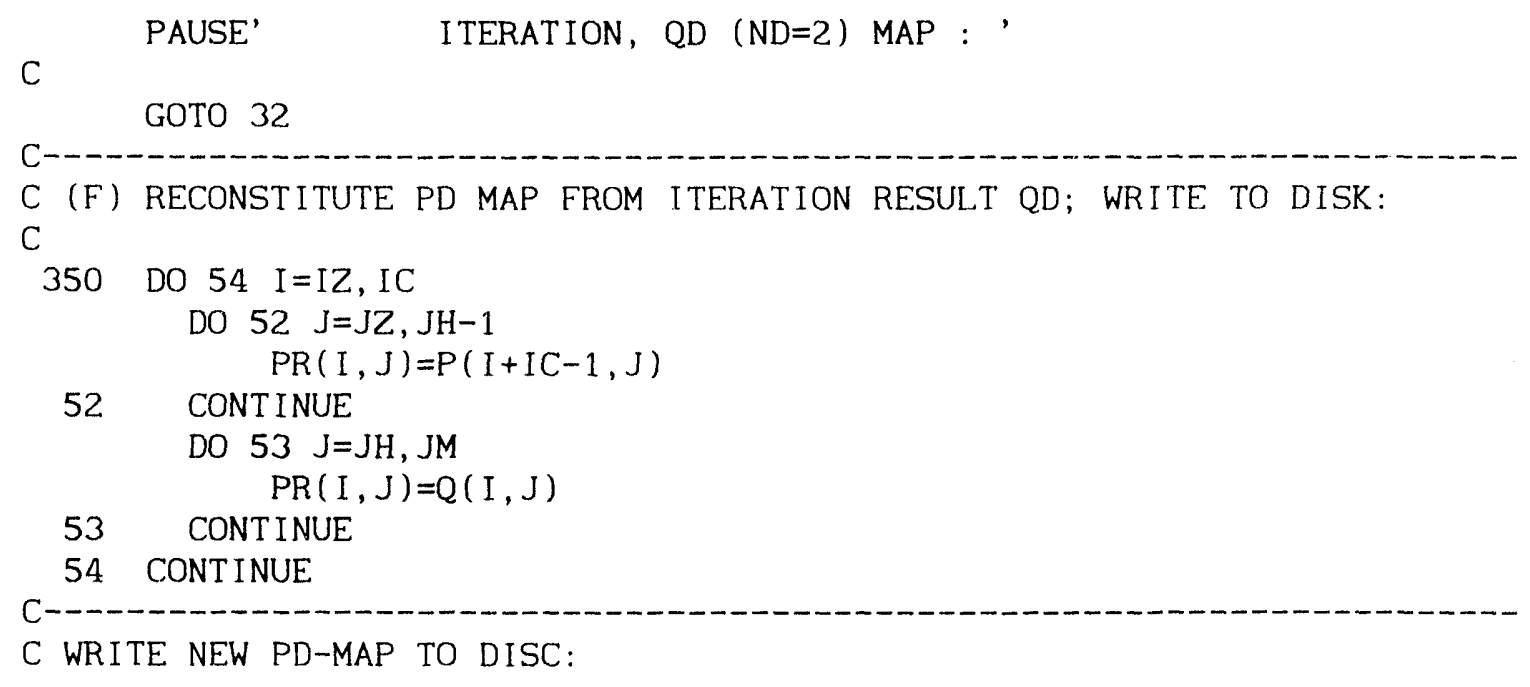

(omitted)

500 STOP

(FORMAT Statements omitted)

END

C WP2E.FOR: Lin. equipotential interpolation, Wrapped Plate geo. Reads

C P-MAP.XXX, combd from PS, PD; scales $x 20$; sets $y$-scale, interpolates

$C X(K, N), Y(K, N)$ files, $1<N<19$. Repts. interpol on QI map (cf WP1E2.FOR)

(Initial statements omitted)

DATA IZ, I I , IA , IC, IB, IM, JZ, JH, JM, NL., NR/1 , 5, 26, 31, 56, 61, 1, 21, 41, 0, 1/

DATA N, YY(1), DYY (1), R(1), R(2)/0,-50. 50.,1.92874985E-22, 1.0/

C1 SETUP: cf, previous Programs, WP.

$Y Y(2)=0$

WRITE $(*, 97)$

DO $1 \mathrm{~J}=2, \mathrm{JM}$

$R(J+1)=F L O A T(J)$

$Y Y(J+1)=\operatorname{ALOG}(R(J+1))$

$\operatorname{DYY}(J)=Y Y(J+1)-Y Y(J)$

$\operatorname{WRITE}(*, 84) \mathrm{J}, R(\mathrm{~J}), Y Y(\mathrm{~J}), \operatorname{DYY}(\mathrm{J})$

1 CONTINUE

$\mathrm{C}$

C2 (a) READ BACK P-MAP. XXX (LEFT HALF=PS, RIGHT HALF=PD( INV);

(omitted)

C (b) ADD BOTTOM ROW, SCALE BY 20:

DO $107 \quad I=I Z, I M$

$\mathrm{Q}(\mathrm{I}, 1)=0$

DO $106 \mathrm{~J}=2, \mathrm{JM}$

$Q(I, J)=20 . * P(I, J)$

106 CONTINUE

107 CONTINUE

C DEF INE DQKX, DOKY: 


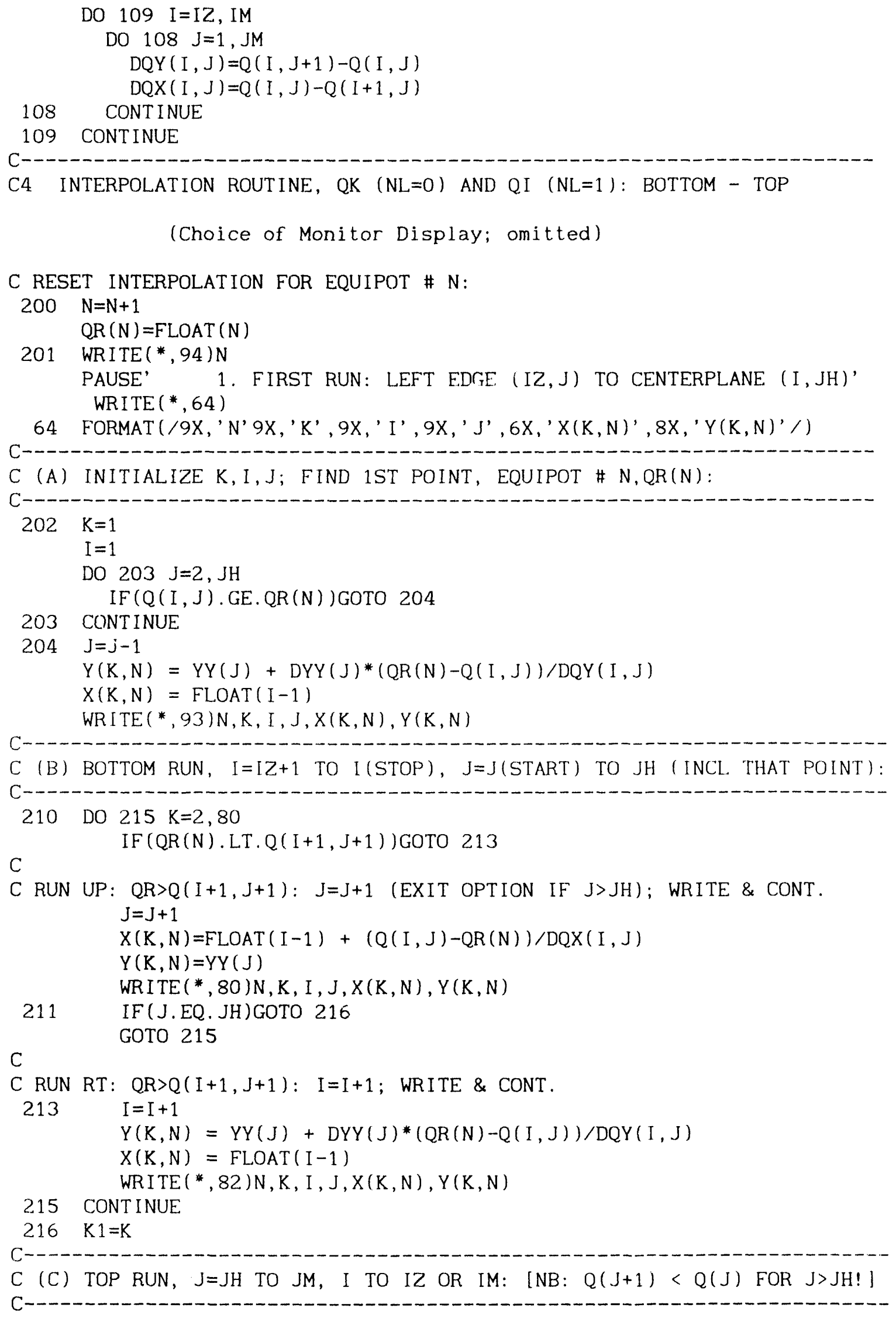




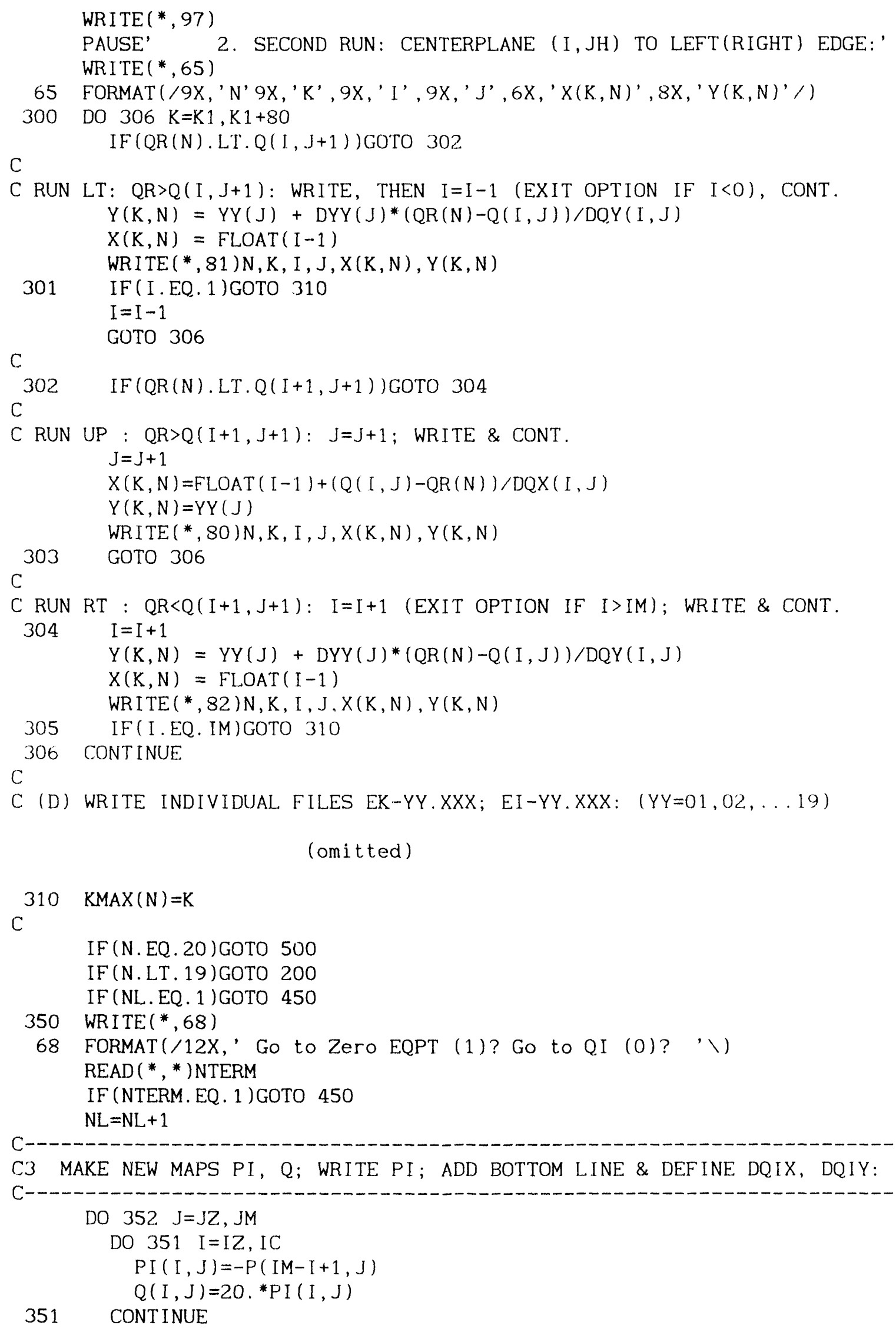


352 CONTINUE

C ( $f$ ) WRITE PI-MAP TO DISC:

\section{(omitted)}

C (g) ADD BOTTOM ROW; DEFINE DQIX, DQIY, DQKX, DQKY

DO $410 \quad I=I Z$, IC

$\mathrm{Q}(\mathrm{I}, \mathrm{JZ})=0$

DO $409 \mathrm{~J}=1, \mathrm{JM}$

$\operatorname{DQY}(I, J)=Q(I, J+1)-Q(I, J)$

409 CONTINUE

410 CONTINUE

$\mathrm{N}=\mathrm{O}$

GOTO 150

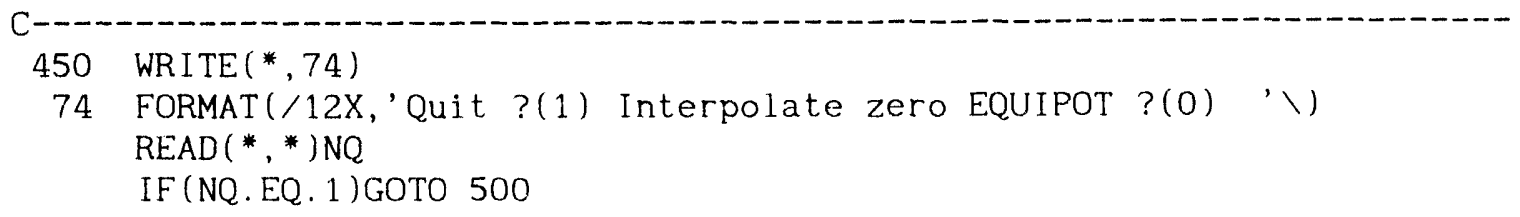

C (h) ZERO EQUIPOTENTIAL INTERPOLATION FROM P-MAP:

$\mathrm{N}=20$

$\mathrm{QR}(\mathrm{N})=0.0$

$X(1, N)=$ FLOAT $($ IC -1$)$

$Y(1, N)=-20$.

DO $453 \mathrm{~J}=2, \mathrm{JH}$

DO 451 I=IC-1, IM

$\operatorname{IF}(P(I, J) . L T . Q R(N))$ GOTO 452

451 CONTINUE

$452 \quad \mathrm{I}=\mathrm{I}-1$

$\mathrm{K}=\mathrm{J}$

$Y(K, N)=Y Y(J)$

$X(K, N)=\operatorname{FLOAT}(I-1)+P(I, J) /(P(I, J)-P(I+1, J))$

453 CONTINUE

WRITE $(*, 80) \mathrm{N}, \mathrm{K}, \mathrm{I}, \mathrm{J}, \mathrm{X}(\mathrm{K}, \mathrm{N}), \mathrm{Y}(\mathrm{K}, \mathrm{N})$

$\mathrm{J}=\mathrm{JH}$

DO $455 \mathrm{~K}=\mathrm{JH}+1,80$

IF $(P(I+1, J+1) . G T . Q R(N))$ GOTO 454

C GO UP:

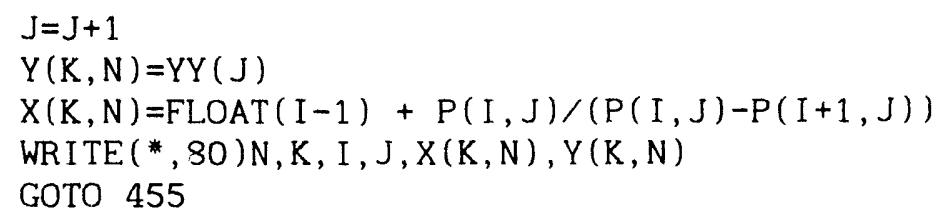


75 FORMAT(/12X,' Declare filename EK-20.XXX ' \)

GOTO 320

(FORMAT Statements omitted)

500 STOP

END

C WP3E.FOR: CONFORMAL TRANSFORM of Cartes. $X(K, N), Y(K, N)$ equipotentials,

C $N=1,2, \ldots 19, K=1,2, \ldots \operatorname{KMAX}(N)$ to $X T, Y T$; also XI,YI TO XIT, YIT. The $x f d$

$C$ files are reset for combined GRAPHER plotting, $1 / 2$ circle. 27/09/92.

(Initial Statements omitted)

DATA IZ, IC, IM, JZ, JH, JM, N, NL, A, PI/1,31,61, 1,21, 41, 0, 0,

X $0.0523598776,3.14159265$

C-1..-

C READ BACK-even-numbered $X, Y$ FILES $N=2 \ldots 20$, one at a time:

(omitted)

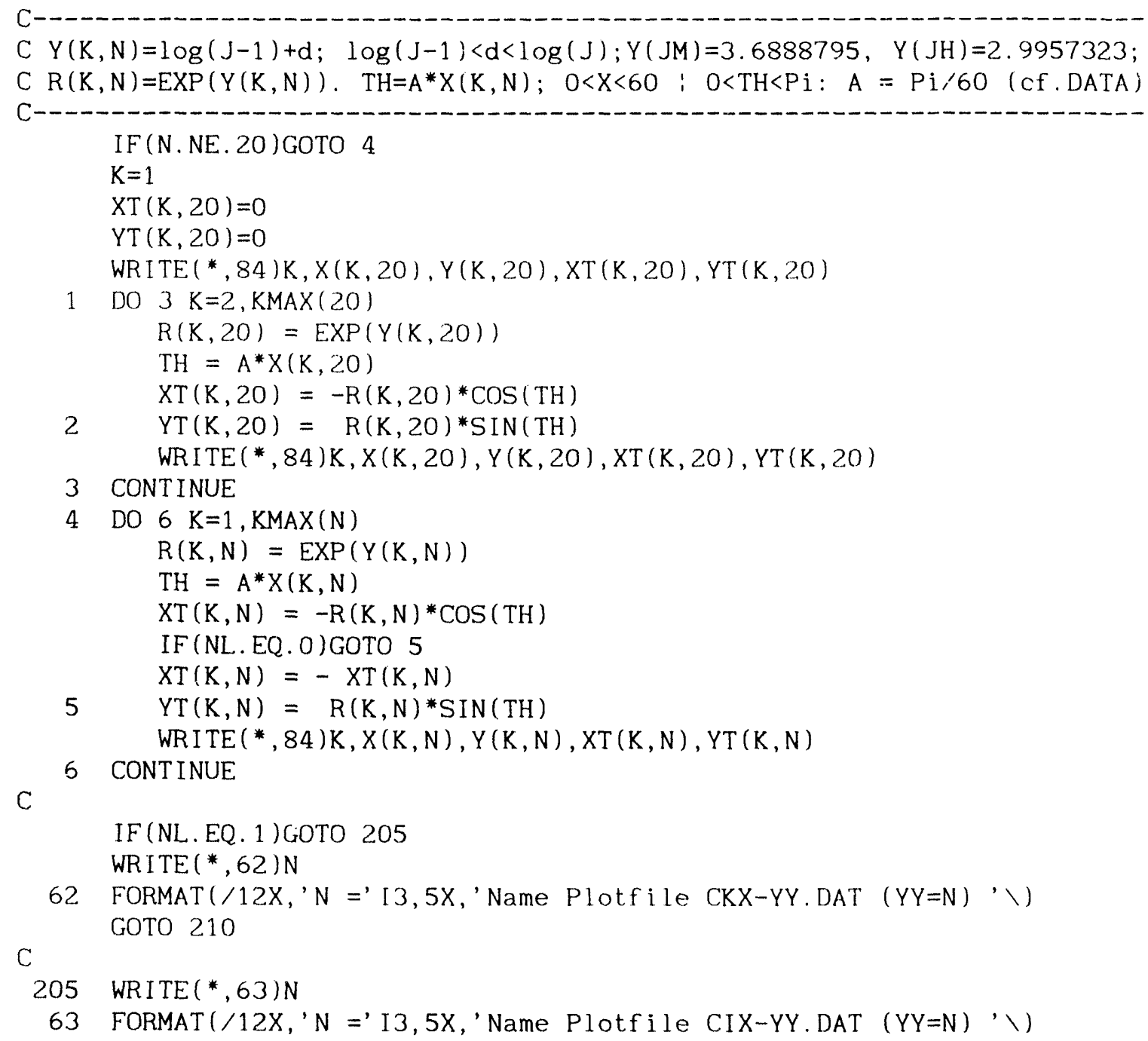




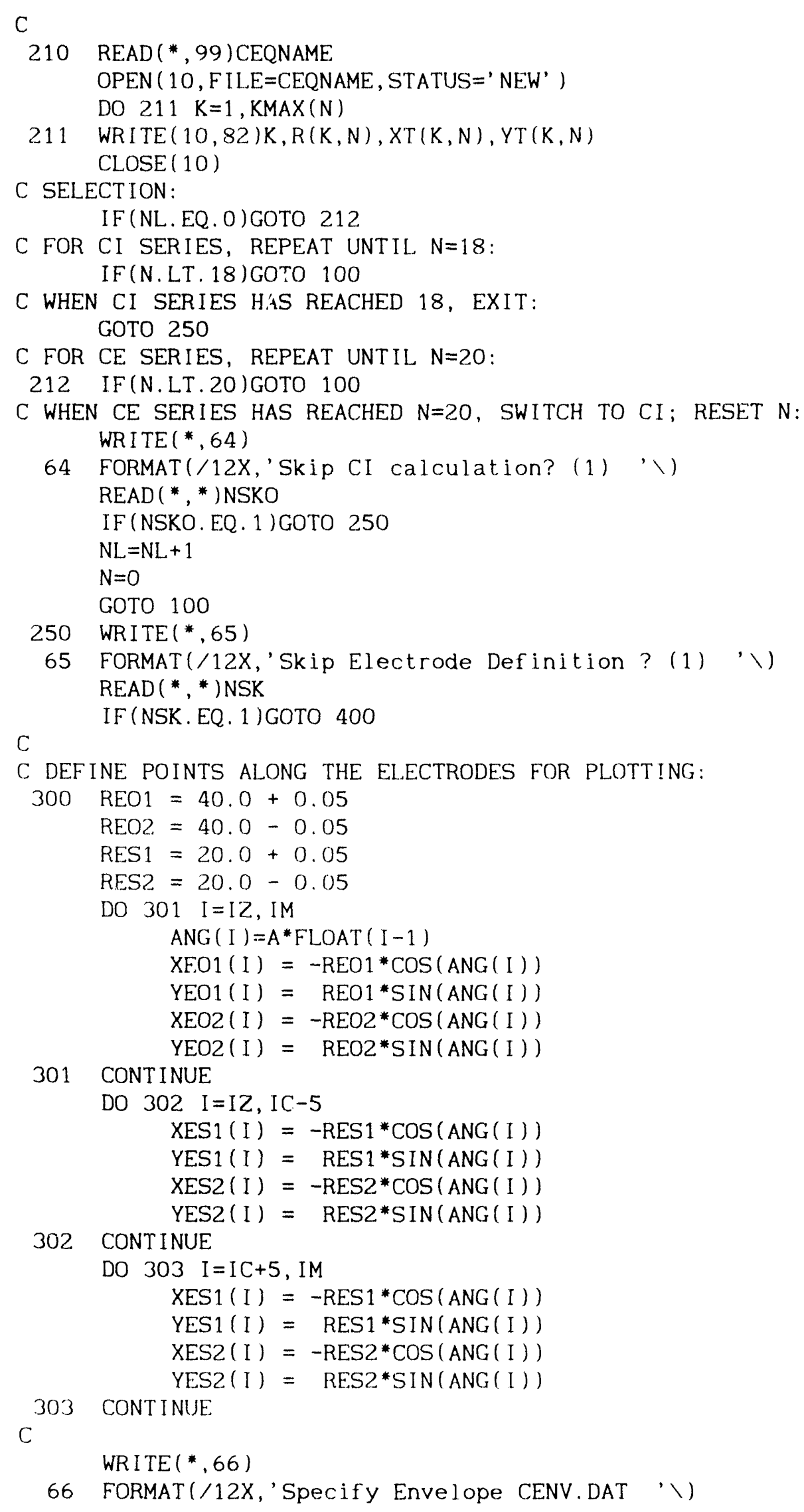




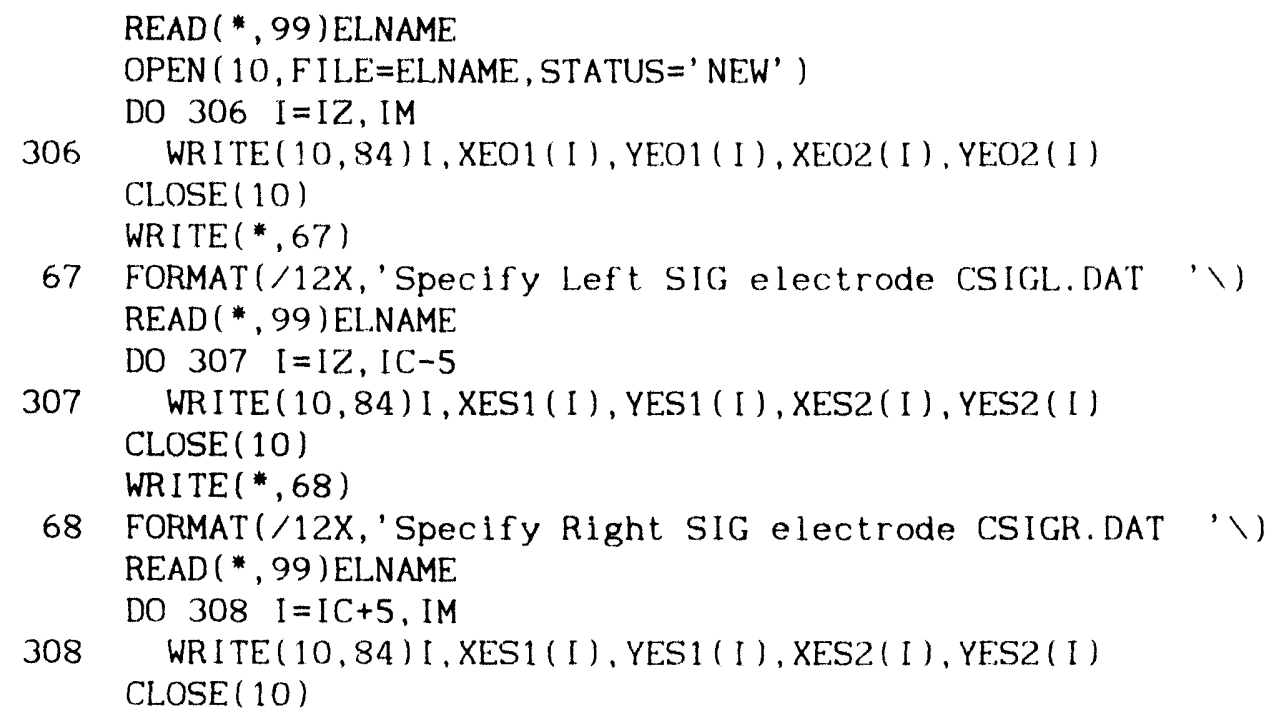

Of the many other programs that were written for this study, the most important comprise a set that calculates the electric field in a rectangular (square) duct, deliberately distorted to produce a viewed slice enrompassing the "specific" velocity contours that the above Navior-Stokes solution programs calculate for laminar flow. The structure of the successive-approximation and equipotential components of this series is similar to the foregoing and therefore omitted here. The program that calculates field lines has perhaps some novelty and is thus reproduced below; it is applicable to circular geometry (in the preliminary Cartesian version).

C CIRPOT3.FOR...draws FIELD LINES from arbitrary pt on SIG electrode *

REAL*8 X(49,41),Y(49,41), XF (25,33), YF $(25,33), \operatorname{TF}(25,33), F X(33,25)$, $X$ FY $(33,25), D X(81), D X L, X U, Y U, T U, X L, Y L, T L, S, D, D O N, Q P, D E L T, H, H A, B$, $X$ FU, FL, DEN, DAN, EMMA, EMMY, G, TB, TFX, A1, A2, A3, A4, B1 , B2, B3, B4, DIT, $X$ DOT, DEXKA, BITKA, XR (132), YR( 132$), \operatorname{ARA}(33,10,8)$

INTEGER IRE, ILE, IWID, NPR, JTOP, JZ, IMAX, JMAX, MAX (33)

CHARACTER*16 CIR2NAME, CFDAT, CFNUM

DATA/IMAX, IEDG, JMAX, JTOP, DEL.Y, DELX, PI , D1, D2/51, 34, 50, 33, 0.03125 , X $0.03125,3.141592654,0.510321632,0.755160816 /$ 


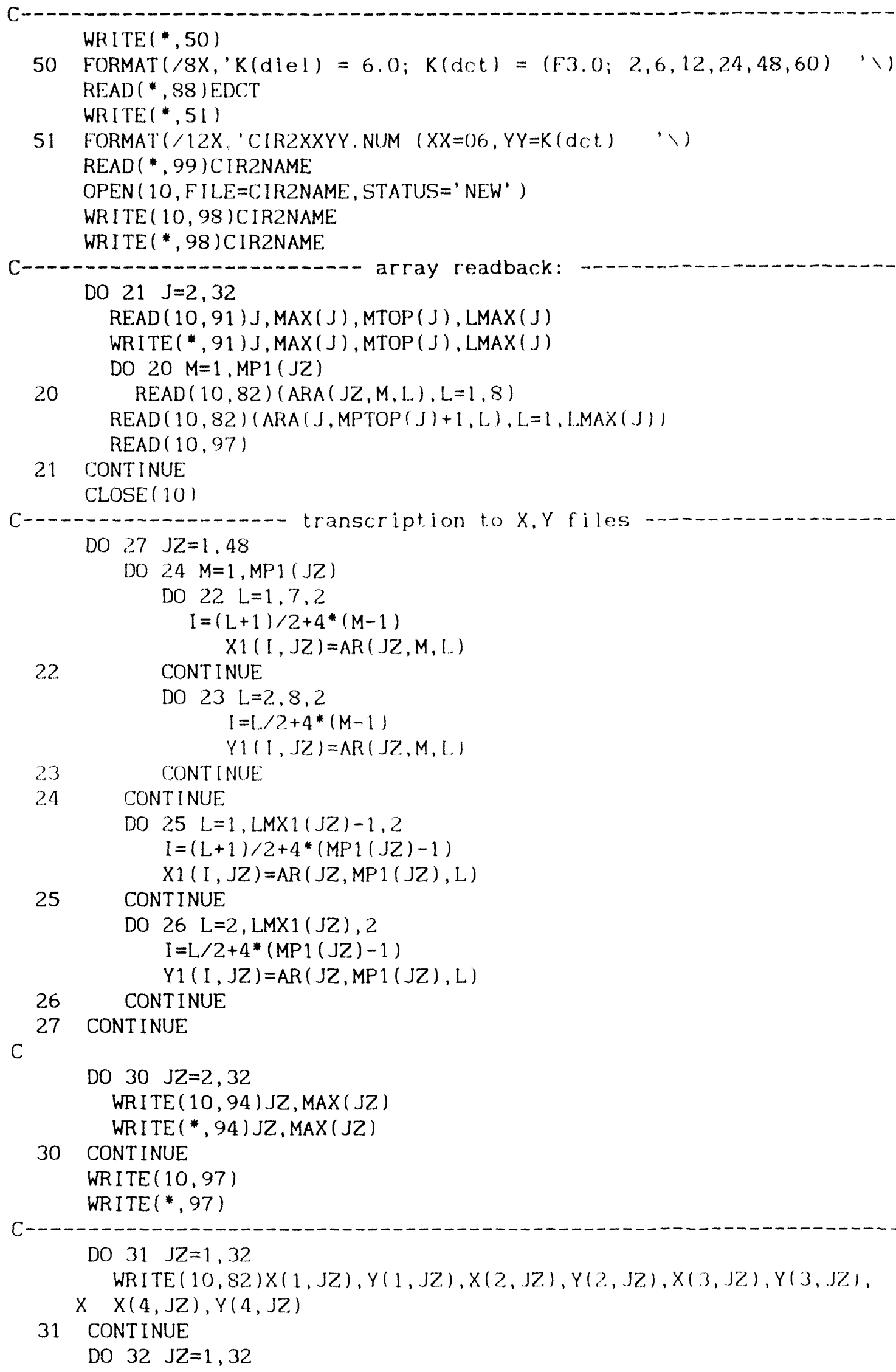




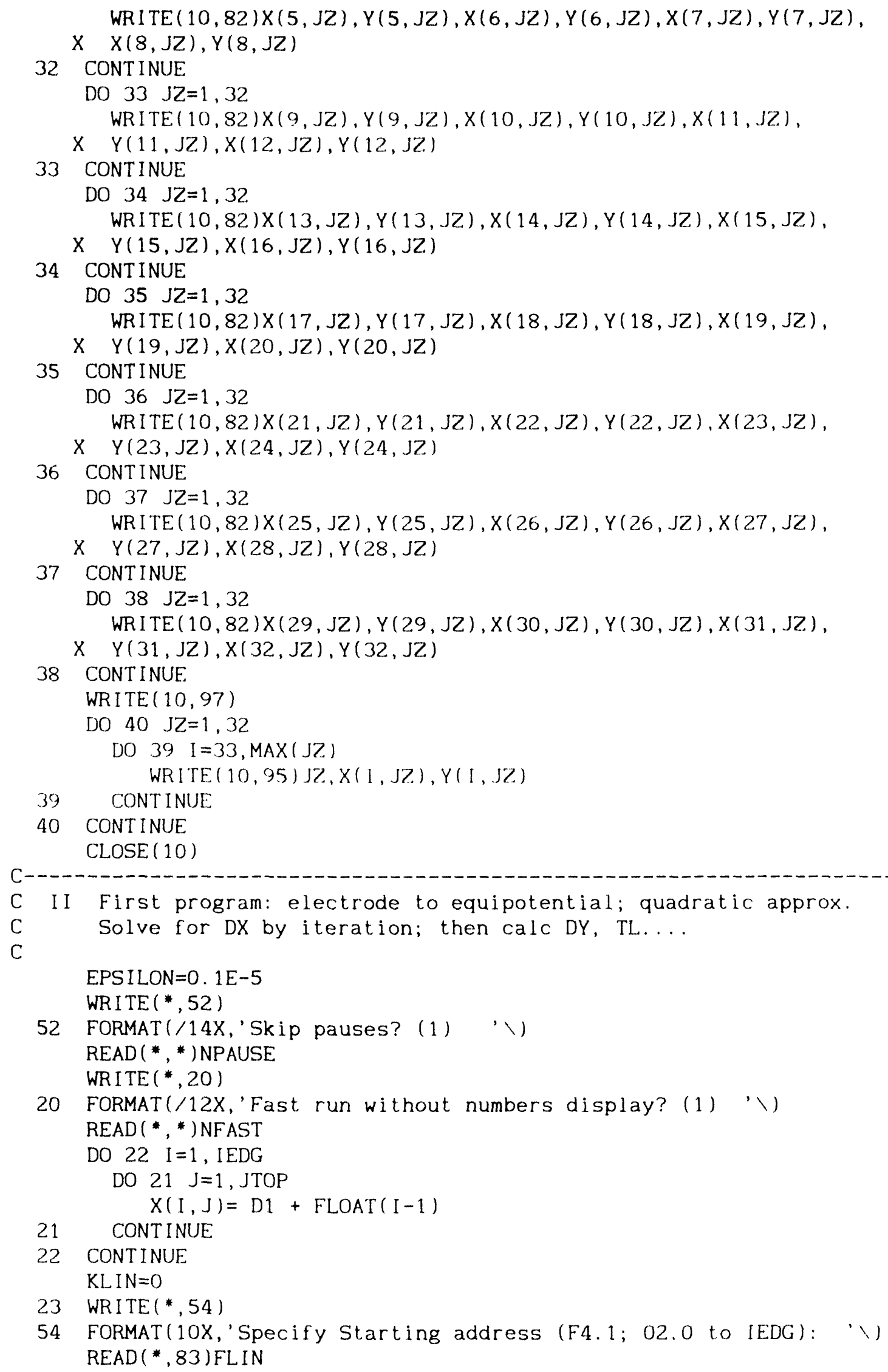




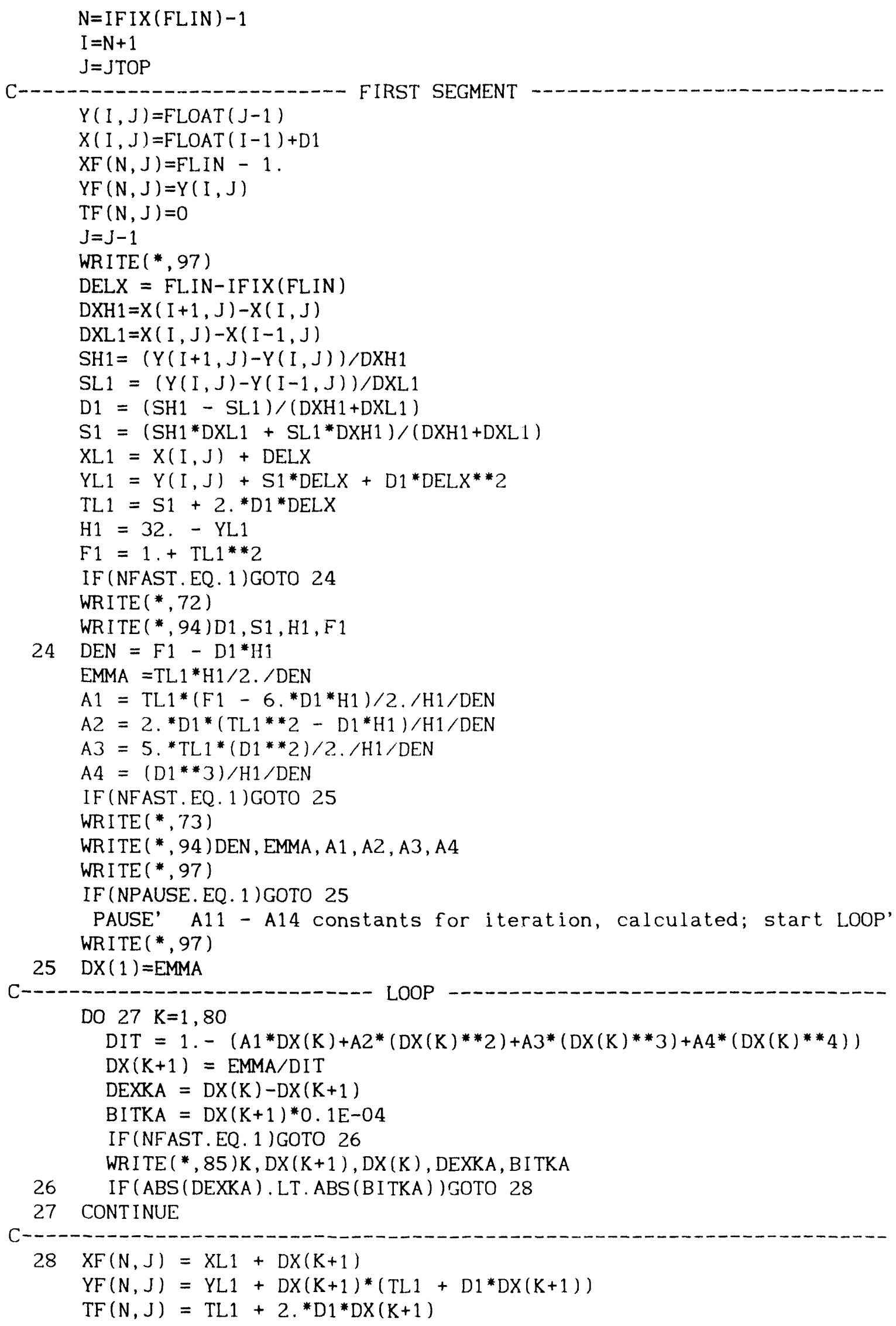


$\mathrm{L}=\mathrm{JTOP}-\mathrm{J}$

IF (NFAST. EQ. 1 )GOTO 56

WRITE $(*, 92)$

WRITE $(*, 96)$ XLL, YLL, S1

WRITE $(*, 90) N, J, L, X F(N, J), Y F(N, J), T F(N, J)$

C III Second Program: Field lines connecting adjacent eqpts; draw norC mal to upper eqpt, find XL, YL intersect on lower eqpt. Quadratic

C approx. Next, find DX by iteration, same Quad approx.

29 WRITE $(*, 97)$

IF (NPAUSE. EQ. 1 )GOTO 56

PAUSE' 2

START NEXT POINT PARAMETER CALCS'

WRITE $(*, 97)$

WRITE $(*, 55)$

55 FORMAT (/16X,'QUIT?(1) ')

$\operatorname{READ}(*, *)$ NQUIT

IF (NOUIT.EQ. 1 ) GOTO 100

$56 \mathrm{XU}=\mathrm{XF}(\mathrm{N}, \mathrm{J})$

$Y U=Y F(N, J)$

$\mathrm{TU}=\mathrm{TF}(\mathrm{N}, J)$

$J=J-1$

WRITE $(*, 97)$

$1 \quad \mathrm{DXH}=\mathrm{X}(\mathrm{I}+1, \mathrm{~J})-\mathrm{X}(\mathrm{I}, \mathrm{J})$

$D X L=X(I, J)-X(I-1, J)$

$\mathrm{SH}=(Y(I+1, J)-Y(I, J)) / D X H$

$\mathrm{SL}=(Y(I, J)-Y(I-1, J)) / D X L$

$D=(S H-S L) /(D X H+D X L)$

$\mathrm{S}=\left(\mathrm{SH}^{*} \mathrm{DXL}+\mathrm{SL}^{*} \mathrm{DXH}\right) /(\mathrm{DXH}+\mathrm{DXL})$

$\mathrm{QP}=((Y U-Y(I, J)) * T U+X U-X(I, J)) /\left(1+S^{*} T U\right)$

DON $=0.500 *\left(1 .+\mathrm{S}^{*} \mathrm{TU}\right) /\left(\mathrm{D}^{*} \mathrm{TU}\right)$

$\mathrm{B}=2 \cdot{ }^{*} \mathrm{QP} / \mathrm{DON}$

IF (NFAST. EQ. 1$)$ GOTO 2

WRITE $(*, 70)$

WRITE $(*, 94) D, S, Q P$, DON , B

$2 \mathrm{DXL}=\mathrm{QP} *(1 .-0.250 * \mathrm{~B}+0.125 * \mathrm{~B} * 2-0.078125 * \mathrm{~B} * 3+0.0546875 * \mathrm{~B} * 4)$

GOTO 3

IF (NFAST.EQ. 1 )GOTO 4

3 WRITE $(*, 80) \mathrm{N}, \mathrm{J}, \mathrm{DXL}$

4 IF (DXL.LT. 1.) GOTO 5

$\mathrm{I}=\mathrm{I}+1$

WRITE $(*, 97)$

IF (NPAUSE. EQ. 1 )GOTO 1

PAUSE'

GOTO 1

DXL $>1: \quad \mathrm{I}=\mathrm{I}+1$; REPEAT DXL CALCULATION'

$5 \quad \mathrm{XL}=\mathrm{X}(\mathrm{I}, \mathrm{J})+\mathrm{DXL}$

$Y L=Y(I, J)+S^{*} D X L+D^{*} D^{*} X^{*} 2$

$\mathrm{TL}=\mathrm{S}+2 \cdot{ }^{*} \mathrm{D} * \mathrm{DXL}$

DELT $=T L-T U$

IF (NFAST. EQ. 1 )GOTO 6

WRITE $(*, 71) X L, Y L, T L$

$6 \operatorname{IF}($ ABS (DELT).LT.ABS (TU)*.001)GOTO 11

WRITE $(*, 97)$

$H A=Y U-Y L$ 


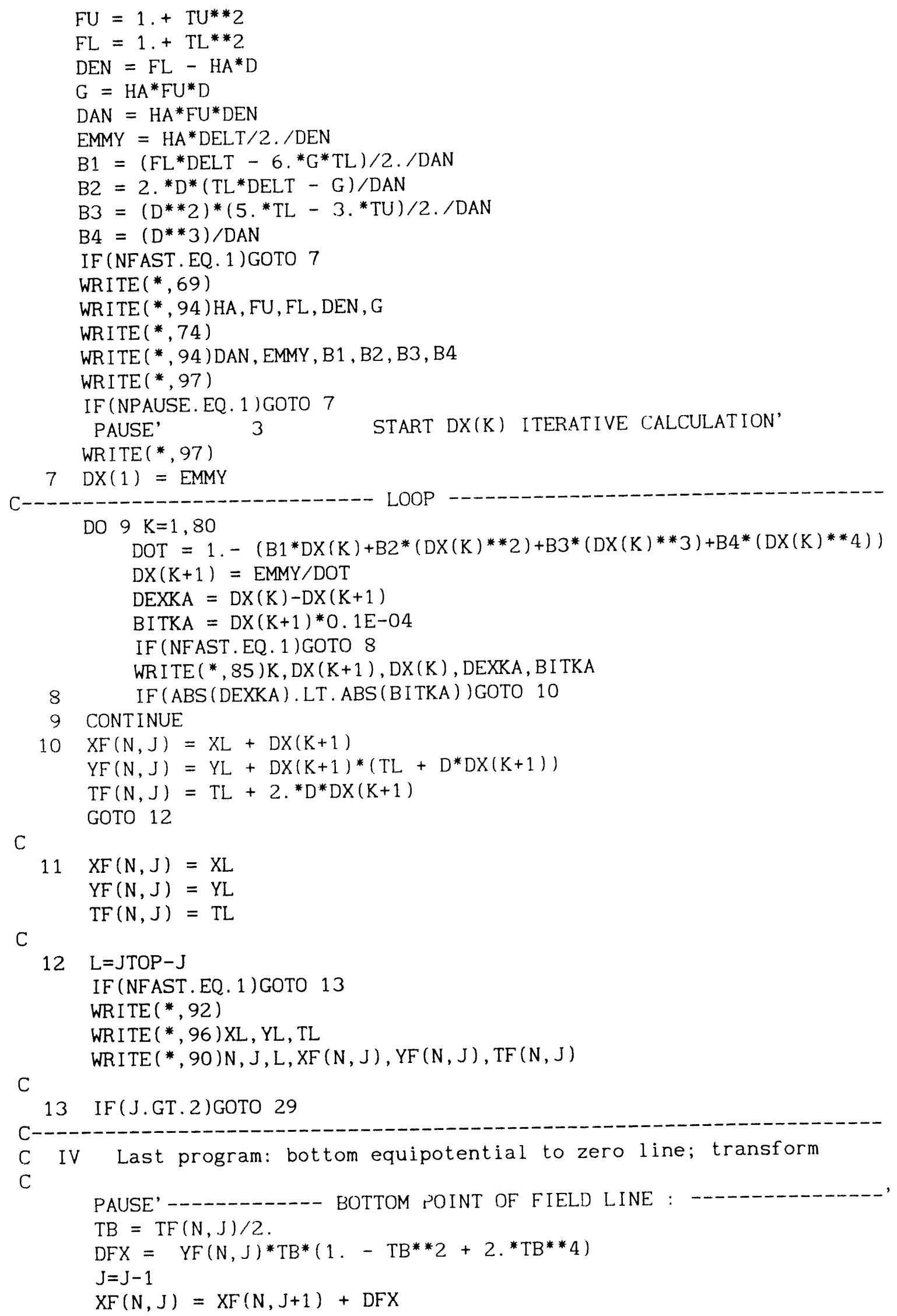


$\mathrm{YF}(\mathrm{N}, \mathrm{J})=0$

$\mathrm{TF}(\mathrm{N}, \mathrm{J})=0$

IF (NFAST.EQ. 1)GOTO 14

$\operatorname{WRITE}(*, 92)$

WRITE $(*, 90) N, I, J, X F(N, J), Y F(N, J), T F(N, J)$

C-..- TRANSFORM FIELD LINE FOR WRITING:

14 DO $15 \mathrm{~J}=1$, JTOP

$F X(J, N)=X F(N, J)$

$F Y(J, N)=Y F(N, J)$

15 CONTINUE

$\operatorname{WRITE}(*, 97)$

$\mathrm{C} \quad \mathrm{V}$ Write Field Line Array to disk

C

WRITE $(*, 88) \operatorname{EDCT}$

WRITE $(*, 58)$

58 FORMAT (/8X,' Specify Line FCXXYYNN. NUM ( $X X=06, Y Y=E D C T, N N=\#$ ' $\backslash$ )

$\operatorname{READ}(*, 99)$ CFNUM

WRITE $(*, 59)$

59 FORMAT (/8X,' Specify Line FXXYYNN. DAT (as above) ' $\backslash$ )

READ (*, 99) CFDAT

$\operatorname{OPEN}(10$, F ILE $=$ CFNUM, STATUS=' NEW' $)$

C WRITE $(*, 98)$ CFNUM

WRITE $(10,98)$ CFNUM

DO $16 \mathrm{~J}=1$, JTOP

16 WRITE $(10,79) J, F X(J, N), F Y(J, N)$

CLOSE (10)

$\operatorname{OPEN}(10$, FILE$=$ CFDAT, STATUS=' NEW' $)$

DO $17 \mathrm{~J}=1$, JTOP

$17 \quad \operatorname{WRITE}(10,95) \operatorname{FX}(J, N), F Y(J, N)$ CLOSE (10)

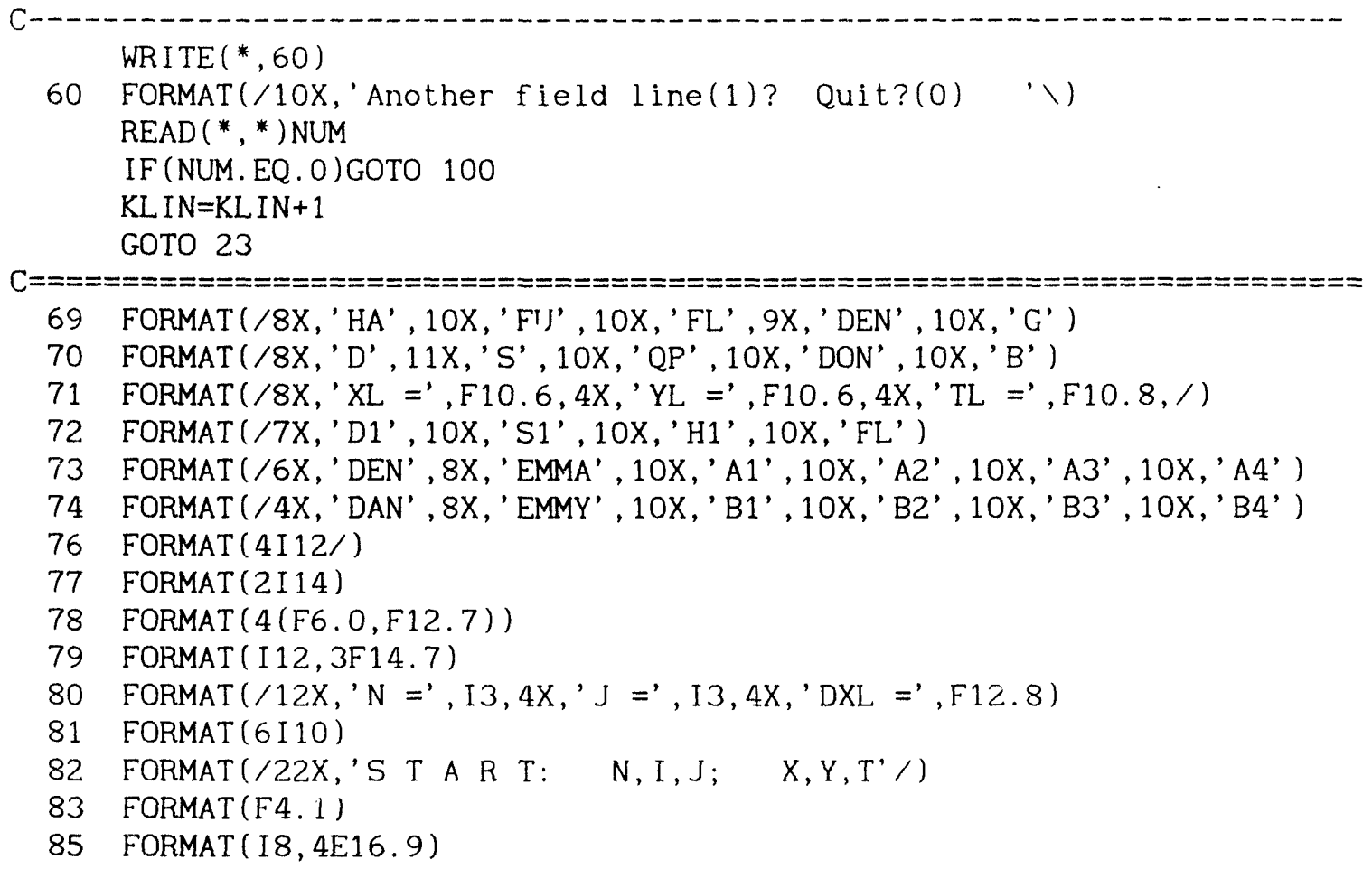




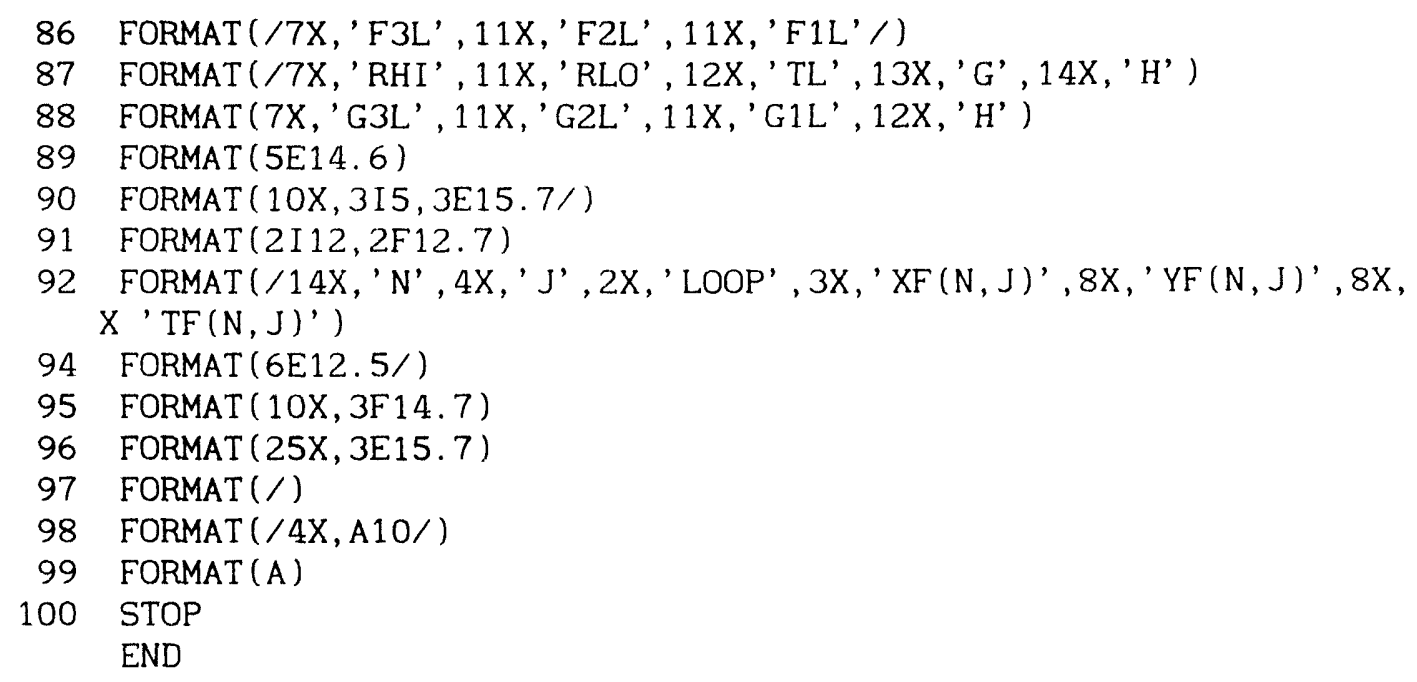

The velocity contour programs (I) can be readily recast to calculate profiles for rectangular rather than square ducts. A more extensive set of changes would be required to represent Bingham Flow.

Incorporation of dielectric regions (duct wall etc.) into the set of programs that trace equipotentials remains also to be done. Such calculations would be desirable not only for added realism but to investigate the "dielectric mismatch" effect on both coverage and local field strength, discussed in the text (where means to minimize this effect are also suggested).

The Field Line program needs to be applied, principally to produce edgefield maps that, with an additional set of programs (that remains to be produced), are used to calculate the readout error resulting from a specified geometry, phase fraction and set of dielectric constants (fluid, solid and surround). The ciesign of a practical instrument seems to us to require these calculations, rather than just more conventional engineering tests that would inevitably be inconclusive unless testing is preceded by adequate design work.

Programs were written in "Plain Vanilla" FORTRAN. " $C$ " versions, more efficient for computational speed, are amongst those further efforts that had to be relegated to future plans. 


\section{APPENDIX C}

\section{PYCNOMETER DESIGN}

The Database supplied with coal as it is traded includes information that is particularly significant, such as caloric value, ash fraction and sulfur content, but omits other useful information, e.g. density, that is needed for the interpretation of on-line on-line composition measurement of slurry conveyors. In this short note, we shall focus on how accurately a gas pycnometer can provide this information.

A pycnometer consists of a ballast volume $V_{0}$ and chamber of volume $V_{1}$, interconnected with a valve, a gas supply and an accurate pressure gauge (reading to $10^{-4}$ ). Pre-filling the ballast to $p_{0}$ with reference gas (say, helium) and placing a solid sample (ground to appropriate mesh size) of weight $W_{s}$ into $V_{1}$, initially at atmospheric pressure $p_{a}$, the communicating valve is opened and the new pressure $p_{2}$ read. The sample density $\rho_{\mathrm{s}}$ is calculated from

$$
\rho_{\mathrm{s}}=\frac{\mathrm{W}_{\mathrm{s}}}{V_{1}-\mathrm{V}_{0} \Delta \mathrm{p}_{01} / \Delta \mathrm{p}_{1 \mathrm{a}}}
$$

where $\Delta p_{01}=p_{0}-p_{1}, \Delta p_{1 a}=p_{1}-p_{a}$. Subtracting atmospheric pressure from the readings, let

$$
\begin{aligned}
& v_{1}=\beta V_{0}, \\
& V_{s}=\text { sample volume }=V_{1}(1-q)
\end{aligned}
$$

then

$$
v_{1}(1-q)=V_{1}-V_{0}\left(p_{0} / p_{1}-1\right) ; \quad p_{0} / p_{1}=q \beta+1=x
$$

Error propagation, applied to $\mathrm{Lq} .(\mathrm{C}-1)$, yields the error in $\rho_{\mathrm{s}}$ :

$$
\varepsilon^{2}(\rho)=\varepsilon^{2}\left(W_{B}\right)+\varepsilon^{2}\left(V_{s}\right)
$$

the latter:

$$
\varepsilon^{2}\left(V_{s}\right)=\left(\Delta V / V_{s}\right)^{2}\left[1+(x-1)^{2}\right]+\left(V_{0} / V_{s}\right)^{2}\left(\Delta p / p_{0}\right)^{2} x^{2}\left(1+x^{2}\right)
$$

in terms of the minimum readable volume and pressure $\Delta p$ and $\Delta V$. Volume is determined by filling with water and weighing, hence both volume minimum readings derive from the same balance sensitivity; pressure readings likewise make use of the same gauge. Let now $\Delta V / \Delta p=m$ and $\left(V_{0} / m p_{0}\right)=Q$, then

$$
\varepsilon^{2}\left(V_{s}\right)=\left(\frac{q \Delta p}{p_{0} Q(x-1)(1-q)}\right)^{2}\left\{1+(x-1)^{2}+Q^{2} x^{2}\left[1+x^{2}\right]\right\}
$$

or ,

$$
\varepsilon^{2}\left(V_{s}\right)=K^{2}\left[\frac{1+2 Q^{2}}{(x-1)^{2}}+\frac{6 Q^{2}}{x-1}+1+7 Q^{2}+4(x-1) Q^{2}+(x-1)^{2} Q^{2}\right]
$$

whe.e

$$
K=\left(\Delta p / Q p_{0}\right)[q /(1-q)]
$$


To choose the optimum ratio $V_{1} / V_{0}$, the above is differentiated with respect to $x-1=u$ and the result set $=0$ :

$$
1+1 / 2 Q^{2}=u\left[u^{2}(1+0.5 u)-1.5\right]
$$

Let $V_{0}=0.51, p_{0}=1 \mathrm{~atm}$ and suppose the balance can read $0.1 \mathrm{~g}$, the pressure gauge $\cong 1 \mathrm{~mm}$ or $0.1 \mathrm{~mm}$, then $\mathrm{m}=\Delta V / \Delta \mathrm{p}=0.1$ or $11 / \mathrm{atm}$,

$$
1+1 / 2 Q^{2}=1.02 ; 3=u\left(u^{2}+0.5 u^{3}-1.5\right) \quad(C-4)
$$

Eq. $(\mathrm{C}-4)$ has the solutions $u=1.2055 ; 1.4423$ hence, $\mathrm{p}_{\mathrm{o}} / \mathrm{p}_{1}=2.2055 ; 2.4423$. For a minimum $q$ of about $0.4-0.3$ (filled - packed volume), the entries in the Table are pertinent.

\begin{tabular}{|c|c|c|c|c|}
\hline q & $\begin{aligned} & \Delta p \\
& \mathrm{~m}=\Delta \mathrm{V} / \Delta \mathrm{p} \\
& \mathrm{Q}=\mathrm{V} / \mathrm{mp}=0.5 / \mathrm{m} \\
& \mathrm{B}=1+1 / 2 \mathrm{Q}^{2} \\
& \mathrm{u}=\beta \mathrm{q} \\
& \mathrm{a}=1+\mathrm{u}^{2} \\
& b=\mathrm{Q}^{2}(1+\mathrm{u})^{2} \\
& c=\mathrm{Q}^{2}(1+\mathrm{u})^{4} \\
& \Delta \mathrm{p} / \mathrm{pQ}=\Delta \mathrm{V} / \mathrm{V}\end{aligned}$ & 0.0002 & $\begin{array}{c}.0001 \\
1 \\
0.5 \\
3 \\
1.4423 \\
3.0802 \\
1.4912 \\
8.8948\end{array}$ & $\begin{array}{c}.001 \\
0.1 \\
5 \\
1.02 \\
1.2055 \\
2.4523 \\
121.61 \\
591.52\end{array}$ \\
\hline 0.4 & $\begin{array}{c}\beta \\
V_{1} \\
q /(1-q) \Delta V / V \\
\varepsilon\left(V_{s}\right)\end{array}$ & $1.3333 E-4$ & $\begin{array}{c}4.808 \\
2.403 \\
3.688 \mathrm{E}-4\end{array}$ & $\begin{array}{c}4.018 \\
2.009 \\
3.564 \mathrm{E}-3\end{array}$ \\
\hline 0.3 & $\begin{array}{c}\beta \\
V_{1} \\
q /(1-q) \Delta V / V \\
\varepsilon\left(V_{s}\right)\end{array}$ & $0.8571 \mathrm{E}-4$ & $\begin{array}{c}3.606 \\
1.803 \\
2.3708 E-4\end{array}$ & $\begin{array}{r}3.013 \\
1.507 \\
2.2928 E-3\end{array}$ \\
\hline
\end{tabular}

GAS PYCNOMETER DESIGN PARAMETERS

If the per cent sample weighing error $\varepsilon\left(W_{s}\right)$ comes to $0.1 \%=10^{-3}$, the overall error still is less than $1 \%$, worst case. However, this is not necessarily so when the dimensions of the pycnometer are poorly chosen, hence, it may be worth while to construct this equipment as suggested in this note. 


\section{Distribution for ANL-EP-TM-93/1}

Internal

R. J. Armani

E. F. Bennett

S. K. Bhattacharyya

W. E. Brewer

E. D. Doss (10)

C. L. Herzenberg

K. K. Larsen

L. G. LeSage

F. R. Lenkszus
W. F. Podolski

K. G. Porges (10)

A. C. Raptis

D. K. Schmalzer

S. H. Sheen

C. E. Till

EP Files

TIS Files

\section{External}

DOE-OSTI (2)

ANL-E Library (2)

ANL-W Library

Manager, DOE-Chicago Operations Office

J. D. Bobis, University of Northern Illinois

T. Byam, Morgantown Energy Technology Center

J. Carr, Office of Fossil Energy, DOE

J. S. Coleman, Office of Energy Research, DOE

A. C. Dolby, EPR.I

N. Dowdy, Pacific Northwest Laboratory

T. M. Ekmann, Pittsburgh Energy Technology Center

H. Feibus, Office of Fossil Energy, DOE

P. Gottlieb, DOE-Chicago Operations Office

D. Hacker, Amoco R\&D Labs

F. Herbaty, DOE-Chicago Operations Office

T. Keech, Morgantown Energy Technology Center

J. Kovach, Morgantown Energy Technology Center

S. Lee, Pittsburgh Energy Technology Center

R. G. Lett, Pittsburgh Energy Technology Center

M. McMillan, Morgantown Energy Technology Center

C. Nakaishi, Morgantown Energy Technology Center

J. Notestein, Morgantown Energy Technology Center

R. R. Schehl, Pittsburgh Energy Technology Center

M. I. Singer, Office of Fossil Energy, DOE

O. Tassicker, EPRI

T. Torkos, Pittsburgh Energy Technology Center 

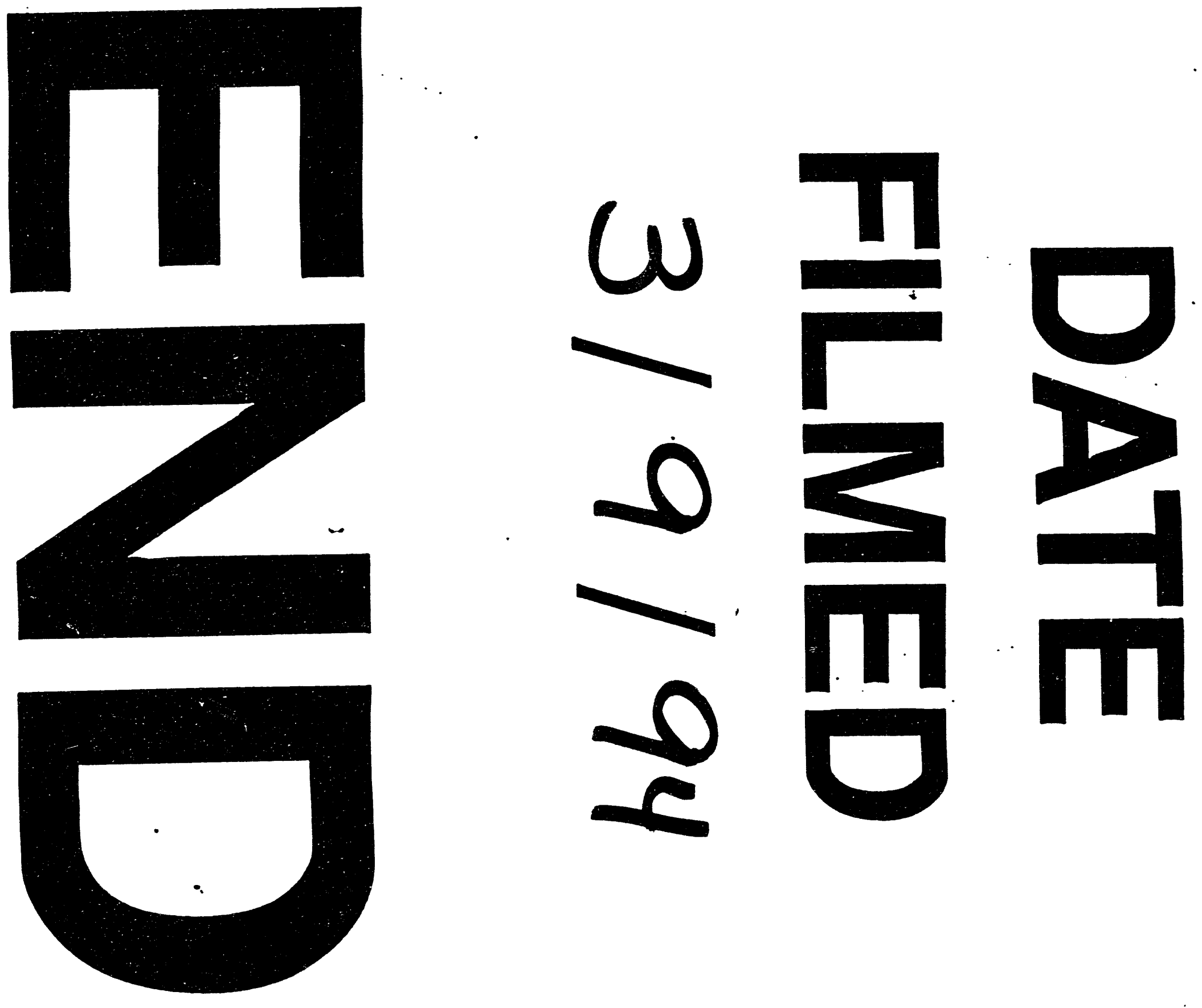
